\title{
Combined creep and hydrogen attack of petro refinery steel
}

By

A. J. Baker

University of Wales Swansea PhD 1999 


\section{IMAGING SERVICES NORTH}

Boston Spa, Wetherby

West Yorkshire, LS23 7BQ

www.bl.uk

The following have been redacted at the request of the university:

Fig 2.2 page 10

Fig 2.3 page 11

Fig 2.4 page 14

Fig 2.6 page 23

Fig 2.7 page 24

Fig 2.9 page 38

Fig 2.10 page 44

Please contact the awarding university for further information. 


\section{DECIARATION:}

This work has not previously been accepted in substance for any degree and is not being concurrently submitted in candidature for any degree.

Signed

(candidate)

Date.

\section{STATEMENT I}

This thesis is the result of my own investigations, except where otherwise stated. Other sources are acknowledged by explicit references. A reference list /bibliography is included

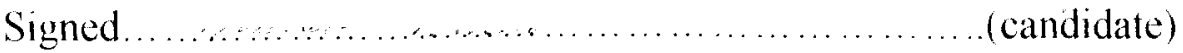

Date

\section{STATEMENT 2}

I hereby give consent for my thesis, if accepted, to be available for photocopying and for interlibrary loan, and for the title and summary to be made available to outside organisations.

Signed (candidate)

Date 


\section{Summary of the Thesis}

\section{"Combined Creep and Hydrogen Attack of Petro-refinery Steel"}

PhD, 1999, University of Wales Swansea

\section{A.J. Baker}

The thesis is based on the study of the interaction between creep and hydrogen attack, with particular emphasis on the commonly used $2.25 \mathrm{Cr}-1 \mathrm{Mo}$ steel. The driving force for the work is the petrochemical industry, where large steel reactor vessels used for hydro-cracking and other processes, are known to sufler damage by hydrogen attack. Currently, no reliable method exists for determining the lifetime of these vessels, due partially to the difficulty of obtaining experimental data under the necessary high hydrogen pressures and temperatures.

A large part of the thesis involved the design and building of unique, high-tech testing facilities which could operate safely and accurately under the severe conditions required for such a study. The test rigs were then used to perform both simple uniaxial creep and internally pressurised tube tests in hydrogen environments. Pressurised tubes were used to provide a stress-state more closely related to that found in industrial components. Such experiments allow more accurate extrapolation to industrial pressure vessel behaviour than would be possible with uniaxial data alone.

The creep results, together with careful metallography, showed the damaging effect of hydrogen attack on creep life and ductility, or conversely, the accelerating elfect of applied stress on hydrogen, attack. Tube testing revealed an effect of the multiaxial stress and demonstrated the importance of the hydrogen concentration in the steel.

The above results were used to develop a Continuum Damage Mechanics (CDM) model for the prediction of tubular behaviour when pressurised with hydrogen. Within the range of conditions studied, the model was successful and further creep testing work is suggested to verify and develop the model further, as well as to study in greater detail the hydrogen attack mechanisms. 


\section{ACKNOWLEDGEMENTS}

The author would like to thank Prof. R. Hurst for his decisive, concise supervision throughout the work programme and Prof. B. Wilshire for help in the final stages of preparing the thesis. He would also like to thank Dr. P. Manolatos for his support, guidance and enthusiasm, and for always finding the time to discuss the ongoing work. A thank you also goes out to $\mathrm{H}$. Rantala for many useful ideas and discussions on creep modelling. Many thanks to Prof. E. van der Giessen and Dr. S. Schloegl for several days of insight at Delft university and help with methane pressure calculations.

The experimental work in this thesis required much technical excellence and innovation. For that, the author wishes to thank Jim Morissey for all his hard work and technical experience in working with high temperature, high pressure hydrogen. Many thanks also to Freek Harskamp for his various technical skills, enthusiasm and countless jokes, to Rene van der Aat for help with creep, and Rob Smit for welding and lifting anything too heavy for a mere mortal human.

A big thank you should also be offered to the metallographic team who were always helpful and prompt with their work; namely, Peter Frampton for preparation and optical microscopy, Kurt Schuster for the SEM, Paul Tambuyser for TEM and Pietro Moretto for image analysis.

Finally, for making the stay in Holland more enjoyable, the author wishes to thank Louise Plunkett for many a delicious meal and other social events, Richard Morgan and Luca Gandossi for being great office mates, and everyone else within the social circle. 


\section{CONTENTS}

1. Introduction

Hydrogen Attack: An Industrial Problem

Plan of the Thesis

2. Literature Review

\section{1}

2.1 Characteristics of Hydrogen Attack

General Mechanisms of Hydrogen Attack

The Stages of Hydrogen Attack

The Methane Reaction

Bubble Nucleation

Bubble Growth Mechanisms

Decarburisation

2.2 Creep

An Introduction to Creep

Creep Deformation Mechanisms

The Effect of Alloying Elements on Creep Deformation

Creep Damage Mechanisms

2.3 Interaction Between Creep and Hydrogen Attack

Mechanistic Interactions in Combined Creep and Hydrogen Attack

Results of Creep-Hydrogen Attack Interation from the Literature

Hydrogen Attack Assisted Creep Crack Growth

\subsection{The Alloy 2.25Cr-1 Mo}

Introduction to the Alloy $2.25 \mathrm{Cr}-1 \mathrm{Mo}$

Microstructure-Property Relations in $2.25 \mathrm{Cr}-1 \mathrm{Mo}$

Creep Behaviour of $2.25 \mathrm{Cr}-1$ Mo Steel

Hydrogen Attack in 2.25Cr-1Mo

2.5 Modelling of Hydrogen Attack

The Need for Hydrogen Attack Modelling $\quad 43$

Hydrogen Attack Modelling in the Absence of External Stress 43

Mechanistic Modelling of Hydrogen Attack Under an Applied Stress 47

Disadvantages of Mechanistic Modelling 48

2.6 Empirical and Phenomenological Creep Modelling 49

Empirical Stress and Temperature Dependence Models 49

Phenomenological Models $\quad 51$

$\begin{array}{ll}\text { Multiaxial Stress Considerations } & 53\end{array}$

Creep Modelling in Aggressive Environments $\quad 60$

$\begin{array}{ll}2.7 \text { Conclusions From The Literature Review } & 61\end{array}$

3. Aims of the Thesis $\quad 63$

$\begin{array}{ll}\text { Main Aims of the Thesis } & 63\end{array}$

$\begin{array}{ll}\text { Methods } & 63\end{array}$

4. Experimental Techniques $\quad 64$

4.1 Material and Specimen Preparation $\quad 65$ 
As Received Material

Uniaxial Creep Specimen Design and Preparation

Tubular Specimen Design and Preparation

Preliminary Test Results and Subsequent Tempering Treatments

\subsection{Uniaxial Creep Testing in Air}

The Uniaxial Creep Rigs

Testing Procedures

4.3 Uniaxial Creep Testing in Hydrogen

Other Project Influences on the Rig Design

Active Gas Containment and Safety in the HCR

Specimen Heating and Temperature Control in the HCR

Creep Specimen Extensometry

Creep Specimen Loading

Testing Procedures

\subsection{Internally Pressurised Tube Testing}

Active Gas Containment and Control in the Tubular Testing Rig

Heating of the Tubular Specimen

Hoop Strain Extensometry

Testing Procedures

\subsection{Test Conditions}

Uniaxial Creep in Air

\subsection{Material Behaviour in Hydrogen Without Stress}

Hydrogen Attack Cavitation in the Absence of Applied Stress

The Surface Decarburised Zone

5.2 Material Creep Behaviour in Air and Argon

Uniaxial Creep in Air

Correlation of Uniaxial Creep in Air, and Argon Pressurised Tubes

5.3 Combined Creep - Hydrogen Attack Behaviour $\quad 125$

$\begin{array}{ll}\text { Uniaxial Creep in Hydrogen } & 125\end{array}$

$\begin{array}{ll}\text { Hydrogen Pressurised Tubes } & 138\end{array}$

Comparison Between Uniaxial and Tube Tests in Hydrogen 151

\section{Creep Curve Modelling}

\subsection{Uniaxial Creep Curve Fitting}

Selection of Modelling Technique

Parameter Fitting

Curve Fits

Alternative Method For Hydrogen Curve Fits

\subsection{Predictions of 'Tubular Hoop Strain Creep Behaviour}

Thick Wall and Thin Wall Stress Distributions 
Hydrogen Pressurised Tubes

6.3 Damage Parameter - Metallography Relations 179

$\begin{array}{ll}\text { Theory and Assumptions } & 179\end{array}$

Techniques for Finding the Ductile and Hydrogen Damage Proportions 179

\begin{tabular}{lr} 
7. General Discussion & 185 \\
\hline
\end{tabular}

7.1 The Effect of Combined Creep Stress and Hydrogen Attack on Cavitation 186

Cavitation Under External Stress Only and Internal Methane Pressure Only 186

Cavitation Under Combined Stress and Internal Methane Pressure $\quad 187$

7.2 The Importance of Surface Decarburisation 192

Surface Decarburisation as a Tool for Understanding Hydrogen Attack. 192

The Effect of Surface Decarburisation in Laboratory Creep Tests $\quad 193$

8. Summary and Conclusions $\quad 194$

$\begin{array}{ll}\text { 8.1 Main Conclusions } & 194\end{array}$

$\begin{array}{ll}\text { 8.2 Suggestions for Further Work } & 197\end{array}$

Appendix A: Surface Changes on Internally Pressurised Tubes 199

Appendix B: Capacitive Hoop Strain Measurement Validation 202

Appendix C: Parameter Fitting of the CDM $\varepsilon^{-\mathrm{m}}$ Model 203

Appendix D: Calculation Procedure of The Thick Walled Concentric Cylinder Model for Predicting Hydrogen Pressurised Tube Creep Behaviour. 206

Appendix E: Determination of the Methane Pressure 210

$\begin{array}{ll}\text { References } & 213\end{array}$ 


\section{INTRODUCTION}

\section{Hydrogen Attack: An Industrial Problem}

At the beginning of this century, Haber and Bosch were working in Germany on the development of a process to produce ammonia from its' constituent elements, hydrogen and nitrogen. In 1908 they built a reactor vessel from carbon steel in which hydrogen and nitrogen were heated under elevated pressure. Eighty service hours later, the reactor vessel failed. After some experimentation, it was deduced that hydrogen and not nitrogen was responsible for the failure; high temperature hydrogen degradation had been discovered. Eventually, in 1911 satisfactory results were obtained by using a steel with a higher chromium content, and in 1916 the process was industrialised in Germany [DMIC'64].

Why did the hydrogen cause the vessel to fail, and why did the steel containing a higher chromium content perform satisfactorily? These questions have been answered over many years of research into what has now become known as "Hydrogen Attack". Hydrogen attack is a form of material degradation which occurs in carbon and low-alloy steels operating under high temperature, high-pressure hydrogen environments. It is distinctly different from hydrogen embrittlement; its' main characteristic being that the damage caused is a result of the chemical reaction between hydrogen and carbon in the steel to form methane gas:

$$
2 \mathrm{H}_{2}+\mathrm{C} \rightarrow \mathrm{CH}_{4}
$$

Under high temperatures and pressures hydrogen can readily dissociate to atomic hydrogen and diffuse into steel, where it may encounter carbon, either dissolved or in the form of carbides. The methane reaction may then occur wherever there is enough space, generally on grain boundaries. Methane is too large to diffuse through the metal, so a high gas pressure can build up which leads to the growth of microvoids. Once neighbouring voids grow large enough to coalesce, they form microcracks that can seriously degrade the material properties. One way to reduce the rate of damage is to make the methane pressure lower. This is normally achieved by adding alloying elements to the steel such as molybdenum and chromium. These elements are strong carbide formers, and tend to hold the carbon in alloy carbides so that it is not available for the methane reaction, or at least it is not so readily available. In other words, lowering the carbon activity lowers the risk from hydrogen attack. 
Since the early work of Haber and Bosch, many processes in the petrochemical, refinery and chemical industries have evolved which require the presence of a hot, high-pressure hydrogen environment. The heart of such hydroprocessing facilities are thick walled steel vessels in which reactions such as coal liquefaction, hydrogen desulphurisation, fuel processing and methanol synthesis take place [Erwin'82], [API'97]. Understanding of the hydrogen attack phenomena, in particular the role of carbon activity, and improvements in steel making technology have lead to the use of new alloy steels for these reactor vessels. As service conditions became increasingly severe in terms of temperature and hydrogen pressure, the carbon steels were replaced with $0.5 \mathrm{Mo}$ steel, $1.25 \mathrm{Cr}-1 \mathrm{Mo}$, and $2.25 \mathrm{Cr}-1 \mathrm{Mo}$ steels, both for improved hydrogen attack and creep resistance. For future use, there is now much interest in the new generation of $2.25 \mathrm{Cr}-1 \mathrm{Mo}-\mathrm{V}$ and $3 \mathrm{Cr}-1 \mathrm{Mo}-\mathrm{V}$ steels. However, the increased cost of these steels means that for less critical operational conditions, carbon and the lower alloy steels are still used. Despite the improvements in understanding and the newer steels, many failures have still occurred in industrial reactor vessels and their associated pipework as a result of hydrogen attack. The majority of these failures have been documented by the American Petroleum Institute, and have been used to create charts called Nelson curves which plot the average working temperatures and hydrogen partial pressures for each recorded failure [API'97]. Based on these data, lines have been drawn to delineate expected safe and unsafe working conditions for various types of carbon and low alloy steels used for vessel construction. The Nelson curves have become the basis for recommended practice in the choice of steel for elevated temperature hydrogen service.

However, the Nelson curves do not allow for the influence of applied stress, material heat treatment or weidments, all factors which are known to affect the hydrogen attack damage. As a result, failures have still occurred in temperature-pressure regimes that were assumed safe by the Nelson curves. In addition, the curves do not allow for vessel lifetime assessment or for behavioural predictions of new steel grades. Most vessel design is therefore necessarily conservative, meaning much higher costs. These drawbacks have lead to new research based on lifetime prediction for both existing and new steels, driven by the industrial urge to gain profit by minimising vessel wall thickness whilst operating safely at higher temperatures and pressures for increased efficiency. Additional savings are desired by prolonging the lifetime of existing reactors, and by reducing the amount of maintenance needed to ensure safe operation. These advances can only be made by greater understanding of the hydrogen attack mechanisms and by increased data on the material behaviour in hot hydrogen environments. 
Much of the problem with the current gaps in knowledge stems from the difficulty to simulate industrial conditions in the laboratory. The industrial temperatures and pressures vary depending on the steel and process, but range up to approximately $450^{\circ} \mathrm{C}$ and $20 \mathrm{MPa}$ pressure for $2.25 \mathrm{Cr}-1 \mathrm{Mo}$ steel. The volatile nature of hydrogen means that great care must be exercised when performing experiments under such high-pressures at elevated temperature. The result of this is a lack of experimental data, and a corresponding lack of understanding of the detailed mechanisms of hydrogen attack. For example, with the desire to increase operational temperatures comes the problem of creep, yet only a handful of creep experiments have been carried out under a hydrogen environment so a reliable database for design purposes does not exist. This is in spite of the fact that both theoretical considerations and the results to date show that an applied stress can greatly accelerate the rate of damage. Furthermore, without such experiments and their subsequent analysis, theoretical lifetime prediction models will lack important mechanistic data and will have no means of being validated.

\section{Plan of the Thesis}

The work in this thesis is aimed at providing data on the hydrogen attack phenomena with particular emphasis on the commonly used $2.25 \mathrm{Cr}-1 \mathrm{Mo}$ low alloy steel. This steel is one of the most widely used for vessel construction, having both relatively good creep and hydrogen attack resistance. However, the steel is known to become more susceptible to hydrogen attack under conditions of applied stress. As such, it makes a good candidate for study, especially given the industrial desire to increase the severity of service conditions to higher temperatures and pressures, where the combination of creep and hydrogen attack could become more important.

The basis of the thesis will be on studying the interaction between creep and hydrogen attack in both simple uniaxial specimens and in pressurised tubes, which provide a stress-states more closely related to those found in industrial components. Such experiments allow more accurate extrapolation to industrial vessels than would be possible with uniaxial data alone. It is hoped that careful metallographical examination of the test material will allow details of the attack mechanism to be revealed, which can lead to greater understanding and to act as input for mechanistic models which are being developed elsewhere.

Chapter 2 of the thesis contains a literature review of the subjects relevant to this work, namely hydrogen attack, creep, creep-hydrogen attack interaction, the alloy $2.25 \mathrm{Cr}$ - $1 \mathrm{Mo}$ steel, and 
modelling approaches for hydrogen attack and creep. At the end of the literature review, a short appraisal is given of the areas in which more work and understanding are needed. Chapter 3 follows on from this by listing briefly the main aims of the research programme. Experimental work is discussed in chapter 4 , and forms a large part of the thesis, due mainly to the difficulty of performing experiments with hot, high-pressure hydrogen. Chapter 5 then presents and discusses the results in terms of the creep properties and the metallographic damage observations which were made. Chapter 6 develops modelling techniques for predicting the lifetime of pressurised tubular components, and compares the predictions with the experimental data. The results are discussed in chapter 7 , and chapter 8 brings together all the work in the conclusions, with some suggestions for further work. 


\section{LITERATURE REVIEW}

The literature review presented in this chapter has been written to provide a broad background into the subjects relevant to the work in this thesis; being namely hydrogen attack, creep, and the interaction between them. Particular reference has been given to the widely used $2.25 \mathrm{Cr}$ 1 Mo steel which forms a large proportion of the current piping and pressure vessels in industrial use today. Whilst the literature review has been written with certain subjects and goals in mind, the content allows for some appraisal of the areas in which more research is needed to clarify given points. These areas are described briefly at the end of this chapter in section 2.7: "Conclusions From the Literature Review", and form the basis of the aims of the thesis. 


\subsection{Characteristics of Hydrogen Attack}

This section of the literature review aims to provide a broad introduction to the phenomena of hydrogen attack, and to clarify the general characteristics and mechanisms as they are currently understood. More detailed information and examples of specific cases of attack will be looked at in later sections.

\section{General Mechanisms of Hydrogen Attack}

Hydrogen attack is a form of material degradation commonly found in carbon and low alloy steels operating under high pressure $(>3 \mathrm{MPa})$, high temperature $\left(>250^{\circ} \mathrm{C}\right)$ hydrogen environments. It is distinctly different from the more popularly studied hydrogen embrittlement, which occurs due to hydrogen supersaturation at low temperatures. At higher temperatures and pressures, the dissociation of hydrogen gas to atomic hydrogen and its' subsequent solubility in iron is increased [Metals'48]. At discontinuities in the steel, such as grain boundaries and inclusions, the dissolved hydrogen can react with carbon to form methane gas. The methane molecule is too large to diffuse through the steel and thus small, sub-micron, internal bubbles containing a high gas pressure can build up. These bubbles may then grow under the influence of the internal pressure, and in severe cases neighbouring bubbles may coalesce, resulting in the development of numerous internal microcracks that drastically reduce the mechanical properties of the steel.

\section{The Stages of Hydrogen Attack}

Bubble growth and coalescence of the type mentioned above is capable of reducing a wide range of mechanical properties. For example, the room temperature properties measured after high temperature hydrogen exposure, such as tensile strength, toughness and reduction of area, may all be severely degraded. In addition, creep strength measured during exposure may also be reduced. However, the way in which the different properties change with time for given exposure conditions normally follows a regular pattern, consisting of three successive stages (Fig. 2.1)

\section{Stage I}

The first stage, otherwise known as the incubation stage, is a time during which little or no damage is noticed. In certain steels, under given conditions, this may last for several years. 


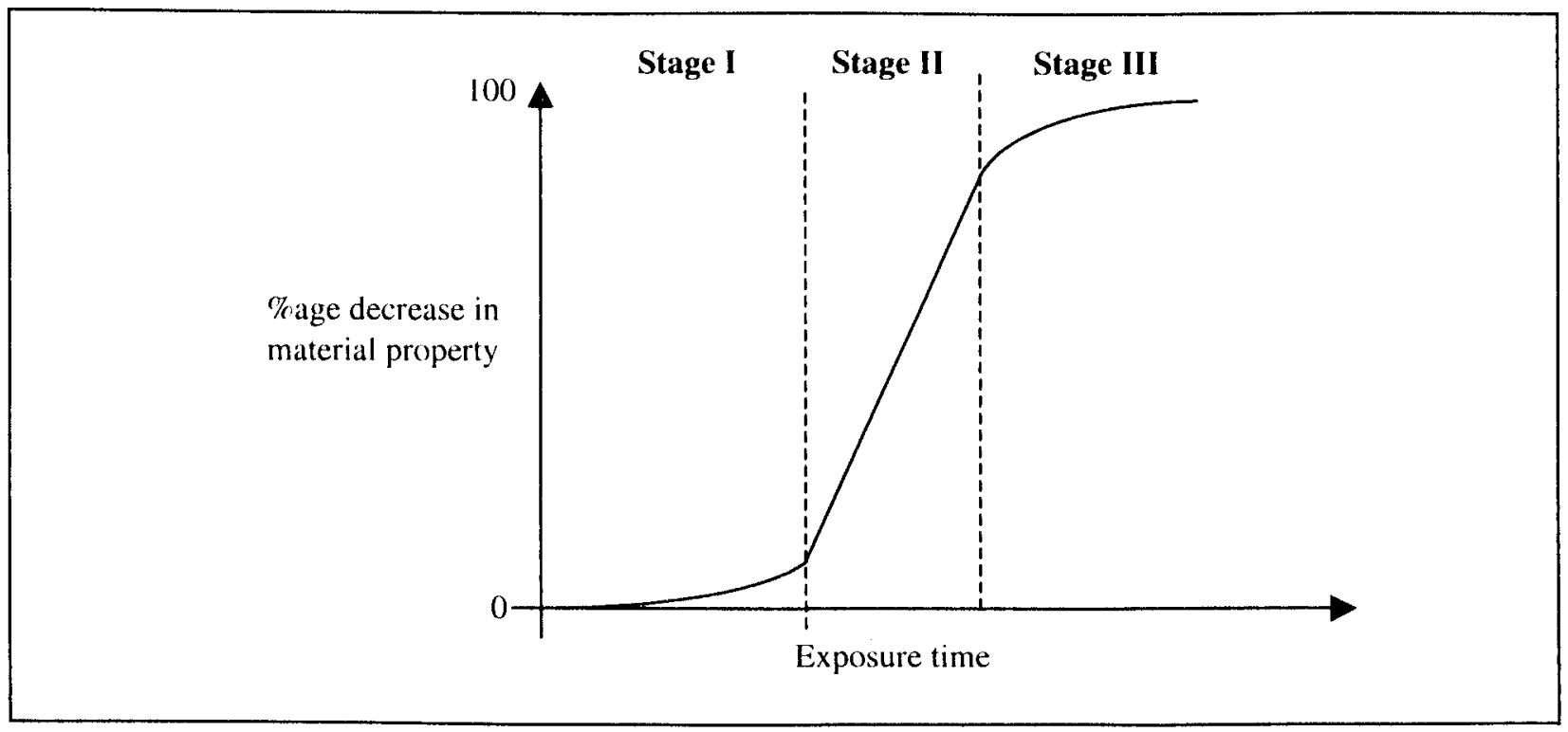

Figure 2.1: Schematic illustration of the three stages of hydrogen attack as a function of material degradation and exposure time

During incubation, small methane filled voids are nucleating and growing due to the reaction between dissolved hydrogen and carbon. Metallographic evidence of this void nucleation and growth has been commonly observed. The length of the incubation stage depends upon the growth rate of the voids, since incubation is considered finished when the voids are large enough to coalesce.

Increasing temperature or hydrogen pressure leads to faster void growth, and therefore to shorter incubation times in carbon steels [API'67]. Cold work prior to exposure can also decrease incubation, with heavy cold reductions (39\%) in carbon steels able to completely remove the incubation stage [Allen'61]. The effect was rationalised in terms of an increase in the number of void nuclei after working. Similarly, in $2 \%$ pre-strained $2.25 \mathrm{Cr}-1 \mathrm{Mo}$ steel, cavities were observed after $350 \mathrm{hrs}$ exposure at $510^{\circ} \mathrm{C}$ in $27.6 \mathrm{MPa}$ hydrogen, whereas non pre-strained material showed no cavities for the same exposure [Woods'83]. Furthermore, the addition of stress to a sample undergoing hydrogen attack can also greatly reduce the extent of incubation [Allen'62]. (More detail on the interaction of hydrogen attack and stress is given in section 2.3 of the literature review). Increases in the incubation period may be achieved by alloying with those elements which lower the grain boundary energy, thereby reducing the number of void nuclei, or which tie up carbon in carbides thus hindering the methane reaction. For example, small additions of Mo in carbon steels have been found to increase the incubation time [Rosent.'63]. Whilst many of the results above were obtained from experiments on carbon steels, they are generally accepted to apply to other steel grades as well. 


\section{Stage II}

Stage two, the "rapid attack" stage, involves a sudden and often severe decrease in material properties over a relatively short period of time. The onset of the rapid attack stage coincides with the link-up of the growing grain boundary voids nucleated during the incubation stage. This leads to internal microcracking and fissuring which quickly degrades the material properties. The damage mechanism is essentially a reduction of internal area, rather than an inherent physical embrittlement of the steel. The rate of damage evolution during rapid attack appears to be dependent upon the same factors as the incubation stage. For example, increasing temperature, pressure or cold work all increase the rate of attack, which suggests that more void nuclei were created, or able to grow under those conditions [API'67].

\section{Stage III}

The third stage shows a decrease in the rate of damage accumulation and may lead to an eventual saturation of degradation. However, by this time the material is usually so badly deteriorated that it is essentially useless, and thus most modelling of hydrogen attack concentrates on predicting the onset of rapid attack (stage 2). Stage three occurs when the methane reaction slows down due to depletion of the carbon within the steel, thereby reducing the driving force for fissure growth. The final extent of damage in the steel depends on the mechanical property being studied, and on the conditions of exposure, with higher temperatures and pressures leading to higher final degrees of attack. Properties such as bending angle to failure and reduction of area seem to be most affected, with degradation of up to 80 or $90 \%$ possible [API'67].

\section{The Methane Reaction}

Since it is the internal methane pressure that drives void growth, it seems reasonable to study the methane reaction itself. Indeed, the calculation of methane pressure is a critical part of most void growth models used to predict hydrogen attack. Methane is the product of the reaction between carbon and hydrogen:

$$
\mathrm{yC}_{(\mathrm{s})}+2 \mathrm{yH}_{2(\mathrm{~g})} \rightarrow \mathrm{yCH}_{4(\mathrm{~g})}
$$

Within a steel however, most of the carbon is tied up in carbides and thus the reaction equation becomes: 


$$
1 / y \mathrm{M}_{\mathrm{x}} \mathrm{C}_{\mathrm{y}(\mathrm{s})}+2 \mathrm{H}_{2(\mathrm{~g})} \rightarrow \mathrm{CH}_{4(\mathrm{~g})}+{ }^{\mathrm{x}} / \mathrm{M}_{(\mathrm{s})}
$$

where $\mathrm{M}_{\mathrm{x}} \mathrm{C}_{\mathrm{y}}$ is the metal carbide present in the steel. This reaction can be split into two parts to simplify the thermodynamic calculations: that of carbide dissolution $\left(\mathrm{M}_{\mathrm{x}} \mathrm{C}_{\mathrm{y}} \rightarrow \mathrm{xM}+\mathrm{yC}\right)$, and that of the methane reaction itself (eqn. (2.1)). However, there is some doubt in the literature as to where exactly the carbon reacts with the hydrogen. For example, some researchers e.g. [Shih' 82] consider that the carbides dissolve into the matrix, releasing carbon that then diffuses to a nearby void where it reacts with hydrogen. Alternatively, others e.g. [VdBurg'96] consider that the two reactions occur simultaneously on the surface of those carbides next to which voids are situated. Despite this, the thermodynamic equations used to calculate the methane pressure follow the same route for each case. The equilibrium methane fugacity (where fugacity is not necessarily equal to pressure) is found from the thermodynamic equation below:

$$
\mathrm{f}_{\mathrm{CH}_{4}}=\mathrm{K}_{0} \frac{\left(\mathrm{a}_{\mathrm{M}_{\mathrm{x}} \mathrm{C}_{\mathrm{y}}}\right)^{1 / y}}{\left(\mathrm{a}_{\mathrm{M}}\right)^{\mathrm{x} / \mathrm{y}}}\left(\mathrm{f}_{\mathrm{H}_{2}}\right)^{2}
$$

where (a) denotes the standard state activity of the metal carbide and the metal, $\mathrm{K}_{0}$ is the equilibrium reaction constant and $f_{H}$, is the hydrogen gas fugacity. Fugacity has units of pressure, but is often greater than the actual mechanical pressure exerted by a gas. The discrepancy arises because of the non-ideal gas behaviour observed at high pressures. Details of the parameters in equation (2.3) are given in the following paragraphs.

\section{Hydrogen Fugacity}

Under most conditions of interest the hydrogen fugacity, $\mathrm{f}_{\mathrm{H}_{2}}$, can be considered equal to the applied hydrogen pressure, provided the pressure is less than about 30MPa [Canjar'67]. Hydrogen is often considered to diffuse into the steel in its atomic form, but re-associates into molecular form at voids and discontinuities where it may subsequently react with carbon e.g. [VdBurg'96].

\section{Methane Fugacity}

The methane fugacity, $\mathrm{f}_{\mathrm{CH}_{4}}$, can be considered equal to the methane pressure only if the pressure is under about $80 \mathrm{MPa}$ [Odette' 82 ]. Above this point, the methane fugacity becomes rapidly greater than the mechanical pressure. This has lead to serious over-estimations of the methane pressure in early models (see for example [Sagues'78]). More recently, an equation of state was 
developed which gives an accurate relationship between the fugacity and pressure (illustrated in Figure 2.2 for $\mathrm{Fe}_{3} \mathrm{C}$ in equilibrium with ferrite) [Odette'82]. The relationship has been simplified into the following equation, which may be solved using numerical root finding techniques:

$$
\mathrm{f}_{\mathrm{CH}_{4}}=\mathrm{P} \cdot \exp [\mathrm{C}(\mathrm{T}) \cdot \mathrm{P}]
$$

where $\mathrm{f}$ is fugacity, $\mathrm{P}$ is pressure, and $\mathrm{C}(\mathrm{T})$ is a temperature dependent constant, the value of which changes with the fugacity range being investigated.

\section{The Equilibrium Reaction Constant}

The value of $\mathrm{K}_{0}$ in equation (2.3) is the equilibrium reaction constant which is dependent upon the free energies of the reacting species. The free energies, and hence $\mathrm{K}_{0}$ values, of various carbides present in alloy steel can be found in the literature (sce for example [Geiger'75], 
[Masaok.'77]), and more detailed work [Lundbe. 77 ] develops expressions for calculating the free energies of alloy carbides from those of the pure metal carbides

\section{Activities}

The final parameters to enter into equation (2.3) are the standard state activities of the metal carbide, $a_{M}, c_{,}$, and the metal, $a_{M}$. In the case of plain carbon steels in which the carbide undergoing dissolution is $\mathrm{Fe}_{3} \mathrm{C}$, the activities $\mathrm{a}_{\mathrm{Fe}, \mathrm{C}}$ of the carbide and $\mathrm{a}_{\mathrm{Fe}}$ of the metal can be considered approximately equal to one [Odette'82]. However, for alloy steels the activities of the carbide and matrix may be different, and must be calculated separately using the activity coefficients for the given alloy system [Geiger'75]. Alternatively, it may be more convenient to express the results in terms of an equivalent carbon activity, $a_{C}$, in solution, such that:

$$
K_{0} \frac{\left(a_{M_{x} C_{y}}\right)^{1 / y}}{\left(a_{M}\right)^{y / y}}=a_{c} K_{C H_{+}}
$$

where $\mathrm{K}_{\mathrm{CH}}$ is the equilibrium methane formation reaction constant. Equivalent carbon activities can be measured experimentally for various alloys by making use of the definition of activity:

$$
\text { Carbon Activity }=\{\text { Carbon Content }\} /\{\text { Carbon Solubility }\}
$$

It has been shown that the lower carbon activities found in well tempered alloy steels produce lower methane pressures and corresponding lower rates of hydrogen attack (Fig. 2.3), 
[Partha.'84]. It was also shown that extremely severe tempering operations would not lower the methane pressure to negligibly small values as predicted by Geiger and Angeles [Geiger'75]. This is because the relatively slow diffusion rate of chromium does not allow for the stable chromium carbides $\mathrm{Cr}_{7} \mathrm{C}_{3}$ and $\mathrm{Cr}_{23} \mathrm{C}_{6}$ to be completely formed.

Detailed calculations of the methane pressure for various alloy carbides in $2.25 \mathrm{Cr}-1 \mathrm{Mo}$ steel have been made, although the lack of thermodynamic data on Mo based carbides was quoted as being an area where inaccuracy was present [VdBurg'96]. It was shown that the equilibrium methane pressure decreases with increasing temperature.

\section{Summary of the Methane Reaction Calculation}

Armed with all the parameters, it is possible to solve equation (2.3) to find the methane pressure which can then be incorporated into hydrogen attack predictive models, although it must be stressed that the calculation of methane pressure is only as accurate as the parameters found in the literature. Further calculations (e.g. [Allen ${ }^{\circ} 62 \mathrm{~b}$ ]) are required if the rate of methane production is to be calculated, with carbon concentration and carbon diffusion becoming important parameters. However, these factors have little effect during the growth of small, discreet voids in the incubation stage, and only really become important as the voids begin to coalesce. Since most models are concerned with predicting the onset of rapid attack, it is often assumed that equilibrium methane pressure is quickly obtained and maintained.

\section{Bubble Nucleation}

Hydrogen attack damage is caused by methane filled voids within the steel. It is therefore important to understand where and why these voids nucleate in order to fully understand the phenomenon. The density of voids gives a good indication of the hydrogen attack susceptibility of a given steel, with high void densities leading to much faster rates of attack.

\section{Nucleation Sites}

Voids nucleate heterogeneously at sites such as grain boundaries, dislocations, sub boundaries and inclusions. The preferred nucleation sites vary from steel to steel. For example, an Al-killed steel may have a preference for grain boundary nucleation [Pishko'79], whilst a similar Si-killed steel may prefer nucleation at inclusions [Ransic.'81]. Voids have also been observed nucleating within the grains [Westph.'74]. Those sites which are "active" in a given steel are determined by the ease with which they can form stable nuclei. Normally, grain boundaries are 
the most common nucleation sites. In order for a suitable heterogeneity to become a void nucleus, there must be enough internal methane pressure to overcome the sintering stress exerted by the surrounding matrix. The driving force for growth $(F)$, of a nuclei has been determined as:

$$
\mathrm{F}=\left(\mathrm{P}_{\mathrm{m}}+\sigma_{\mathrm{n}}-2 \gamma / \mathrm{a}\right)
$$

where $P_{m}$ is the internal methane pressure, $\sigma_{n}$ is an applied stress (or internal residual stress) normal to the grain boundary, $\gamma$ is the void surface tension, and ' $a$ ' is the bubble radius [Shewmo.'85]. It was further suggested that, for values of $\mathrm{F}$ greater than approximately $600 \mathrm{MPa}$, a high density of voids could easily nucleate from heterogeneities throughout the steel, such as is often observed in carbon steels. However, for low alloy steels that exhibit lower methane pressures, the driving force will drop. In this case, only those nucleation sites with a radius bigger than a critical size will form stable nuclei and hence, the density of voids and subsequent rate of attack will be lower.

\section{The Effect of Cold Work on Bubble Nucleation}

Cold work increases the number of nuclei by two mechanisms. Firstly, many more nucleation sites having critical radii may be created by the deformation processes, such as fracturing of inclusions and particle decohesion [Allen'61]. Secondly, large local internal stresses may be built up which can reduce the driving force required for initial growth [Clugst.'83].

\section{The Nucleation Rate}

Void nucleation is often concentrated in small areas within a steel. For example, some grain boundaries may be heavily voided, whilst others appear untouched. When a new void begins to grow, it may do so by diffusion of iron atoms into the grain boundary. This plating of atoms jacks apart the boundary, thereby setting up a tensile stress normal to that boundary. The local stress may then aid in the further nucleation of new bubbles, and so on [Shewmo.' 80]. Thus a continuous nucleation process is predicted with the number of voids increasing with time. Experimental observations in carbon steels back this up [Vitove.'64]. However, in low alloy steels where the critical void radius is greater and the methane pressure smaller, the nucleation rate may quickly fall off as all the pre-existing voids are used up [Sundar.'80]. In this case, it is often assumed for modelling purposes that nucleation is instantaneous and that no new nuclei will be created. 


\section{Bubble Growth Mechanisms}

The following paragraphs describe briefly the main mechanisms by which bubbles may grow. The mechanisms are very similar to those found in creep cavitation (in the absence of $\mathrm{H}_{2}$ ) where the driving force is provided by an external stress as opposed to an internal pressure. More detail of creep cavitation mechanisms can be found in section 2.2 of the literature review.

\section{Diffusional Growth}

Those bubbles that successfully nucleate may continue to grow by two main mechanisms. The first is by diffusion of iron atoms away from the bubbles and into the surrounding grain boundaries. This process is in fact the sum of two diffusional steps. Firstly, surface diffusion of the iron atom along the edge of the bubble, followed by subsequent grain boundary diffusion as the iron atom is moved into the boundary. The relative rates of these two processes determine the shape of the bubble, with crack like bubbles obtained when grain boundary diffusion is dominant, and spherical bubbles obtained when surface diffusion is dominant [Chuang'79]. Higher applied stresses normal to the grain boundary, or higher methane pressures tend to increase the grain boundary diffusive flux, and promote the existence of the crack shaped bubbles [Panda'84]. Diffusional void growth has been calculated to be the dominant mechanism during the early incubation stage of hydrogen attack [McKimp.' 81 ].

\section{Creep Growth}

The second growth mechanism is the expansion of the bubbles by internal pressure driven local creep deformation of the surrounding matrix. Creep growth becomes particularly important as

Figure 2.4: Ductile shearing of ligaments between cavities in a carbon steel after exposure of 24.5 hours at 6.2MPa $\mathrm{H}_{2}$ and $537^{\circ} \mathrm{C}$. (from [API'67]) 
the bubbles become large enough to coalesce at the beginning of the rapid attack stage [McKimp.'81], especially in those steels with lower creep strengths. Evidence of local creep growth can be observed as the remaining ligaments between neighbouring bubbles yield in a ductile manner (Fig. 2.4).

\section{The Effect of Temperature and Pressure on Growth Rate}

In the incubation stage of hydrogen attack, the overall macroscopic growth rate of a specimen due to internal bubble formation has been found to obey the following form of temperature and pressure dependencies [Sundar.' 80 ]:

$$
\frac{\Delta \mathrm{l} / \mathrm{I}}{\Delta \mathrm{t}}=\mathrm{A}\left(\mathrm{P}_{\mathrm{H}_{2}}\right)^{\mathrm{n}} \cdot \exp (-\mathrm{Q} / \mathrm{RT})
$$

where $\mathrm{A}, \mathrm{n}$ and $\mathrm{Q}$ are constants to be determined. Thus, for given exposure conditions, the growth rate of cavities is constant. This has been observed in Ferrovac 1020 and Ferrovac E carbon steels [Westph.74] and in 2.25Cr-1Mo steels [Shewmo.'82]. However, this linear relation quickly breaks down once the incubation stage is over, and the growth rate accelerates rapidly.

Increasing the temperature generally leads to an increase in the bubble growth rate, despite decreasing the equilibrium methane pressure. The most common reason for this is an increase in the diffusion rate at higher temperatures. In addition, creep strength may also be reduced. Bubble growth rates also increases with increasing hydrogen pressure. This can be explained in terms of the extra methane pressure that is generated. Two regions of bubble growth have been identified [McKimp.' 81 ]. At high pressures and low temperatures, growth rate is sensitive to temperature but less sensitive to pressure. The opposite is true for the high temperature, lowpressure regime.

\section{Decarburisation}

\section{Internal Decarburisation}

As the methane reaction continues the amount of carbon remaining in solution, or in carbides, is depleted. Normally the effect of decarburisation would be to reduce hardness and strength, but to increase ductility. Fatigue tests on pre-hydrogen attacked specimens of $\mathrm{Mn}-\mathrm{Mo}-\mathrm{Ni}$ and $2.25 \mathrm{Cr}-1$ Mo steel showed only a slight increase in crack growth rate with increasing amounts of 
HA damage [Pendse'85]. The increased crack growth rate caused by cavitation was offset by a reduction of growth rate due to the increased plasticity at the crack tip resulting from decarburisation. However, because of the numerous voids formed as a result of the methane pressure, the overall effect is normally one of macroscopic embrittlement, despite the decarburisation.

The existence of methane filled voids in commercially pure, low carbon iron suggest that that the amount of carbon required to form small, discreet bubbles during the incubation stage is relatively low. [Yokoga.'89]. However, when larger fissures form during rapid attack then the amount of carbon required increases greatly, and the extent of internal decarburisation can be severe. Indeed, the reduction in the rate of attack during stage three is often caused by complete internal decarburisation. Carbon steels illustrate the above points particularly well, with little or no decarburisation observable around the small voids during incubation and complete internal decarburisation seen in the presence of large voids and fissures [Ransic.'81].

\section{Surface Decarburisation}

Surface decarburisation is caused by the diffusion of carbon to the steel surface where it reacts with the hydrogen atmosphere to form mèthane. In this case, no equilibrium pressure builds up since the methane is free to escape. Thus, the reaction is continuous, with its' rate depending upon the rate of carbon diffusion to the surface. Decarburised layers ranging in size from several microns to a few millimetres have been observed. Theoretical study of the phenomena provides the following important points [Grabke'72]:

1. Decarburisation rate is proportional to carbon activity

2. Higher temperatures lead to increased decarburisation (due to greater carbon mobility)

3. Decarburisation rate is proportional to hydrogen pressure to the power $3 / 2$

Prior work showed that small additions of water to hydrogen gas greatly increase the rate of surface decarburisation [Campbe.'23]. One explanation is that water can dissociate on the metal surface to release oxygen ions that may subsequently react with carbon to form carbon monoxide. This reaction is very rapid compared with the methane reaction, and has both industrial and laboratory significance.

Void formation and surface decarburisation are competing processes [Rosent.'63]. Conditions that favour the surface reaction, such as high temperatures, mean that carbon will diffuse rapidly 
to the surface, leaving a carbon depleted region behind. Void growth in this region becomes retarded by the lack of carbon required to create the high methane pressures necessary for growth. 


\subsection{Creep}

This section of the literature review aims to describe the phenomena of creep in metals. Brief descriptions of the general deformation and damage mechanisms will be given. The following section will then look at the mechanistic interaction that can occur when hydrogen attack takes place in a creeping material.

\section{An Introduction to Creep}

Creep is the time dependant deformation of a material that occurs under a given load. It is a thermally activated process, becoming significant at temperatures above approximately 0.3-0.4 of the absolute melting temperature $\left(T_{m}\right)$ of the material in question. With the use of progressively higher temperatures in industrial plants, creep behaviour has become a critical property of many materials. For example, the increase in superheated steam temperature in power plants over the last half century, from around $750 \mathrm{~K}$ to about $830 \mathrm{~K}$, has lead to the development of low alloy steels to replace the mild steels previously used for steam pipework.

The basic creep characteristics of a material are usually determined by measuring the strain evolution with time of a test specimen exposed to a uniaxial tensilé load. The two most common types of test take place under either a constant uniaxial load, or a constant uniaxial stress. Since stress is defined as the applied load divided by the cross sectional area of the specimen, it is necessary during constant stress testing to gradually decrease the applied load as the specimen stretches and becomes thinner. This may be achieved by either electronically controlled loading devices, or by applying the load via a loading lever with a profiled cam. The resulting creep curves of strain vs. time for the two types of test are shown schematically in Figure 2.5.

These curves are generally split up into various regions. Upon initial loading, instantaneous elastic strain is recorded. Depending on the stress - temperature conditions, plastic strain may also be present. The primary creep stage follows, being characterised by a decrease in the creep rate with time until it reaches some steady state (secondary stage), where the creep rate remains approximately constant for a given time. In the tertiary stage, the creep rate increases until fracture of the specimen intervenes. The prominence of the various stages, and hence the curve shape, varies from material to material and on the temperature and stress applied. Constant load tests, in which the stress is continually rising due to thinning of the specimen, will normally fail 


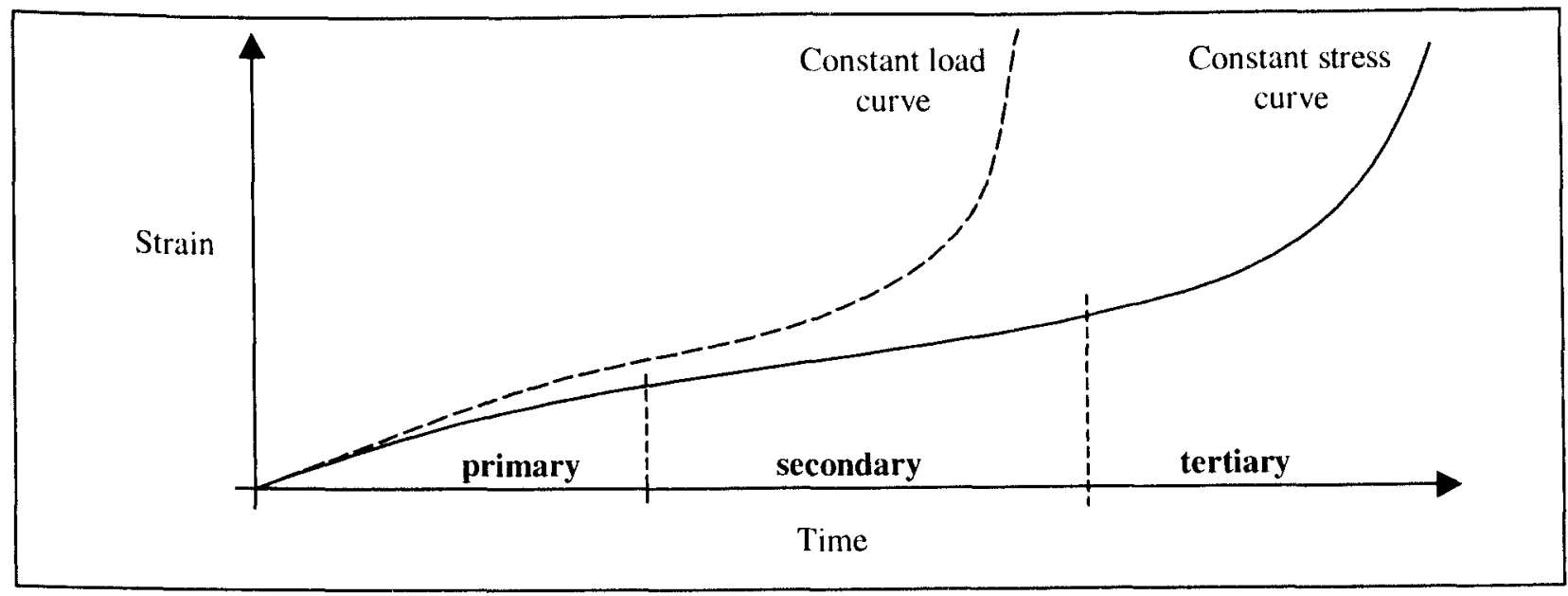

Figure 2.5: Schematic illustration showing the difference between constant load and constant stress creep curves for the same initial stress. Also shown on the constant stress curve are the three divisions normally used to describe the curve shape

sooner, and exhibit greater secondary creep rates than constant stress tests of the same initial stress.

\section{Creep Deformation Mechanisms}

The creep rate during the primary and secondary phases is controtled by hardening and softening mechanisms. In primary creep, strain hardening processes such as dislocation pile ups are dominant, and thus the creep rate decreases. Thermally activated recovery mechanisms act to soften the material, and during the steady state period the hardening and softening rates balance each other out. It has been argued that the nominal steady state creep rate should be referred to as a minimum creep rate, its value being the gradient at the point of inflexion on the creep curve [Evans'82]. In this way, it represents exactly the point when hardening and softening rates are equal. The following presents a brief outline of the main deformation mechanisms found to occur in creeping metals.

\section{Dislocation or 'Power Law' Creep}

Dislocation, or power law creep occurs under high stresses, where the value of a "high stress" is material dependent. It was proposed that dislocation sources continuously emit loops, which glide through the grains, thus producing slip and accumulating strain [Weertm.'55]. As more loops are emitted the leading dislocations may become held up at obstacles, such as immobile Lomer dislocations, and hence a dislocation pile-up occurs behind the obstruction. Pile-ups may also occur due to interaction between mobile dislocations on the same slip plane [Weertm.' 57 ]. 
This leads to a back stress that can prevent the dislocation source from emitting further loops, and is known as the hardening event. However, at temperatures within the creep range, dislocations have an extra degree of freedom called climb. By absorbing vacancies, a dislocation can climb out of its slip plane and into another, thereby overcoming an obstacle in its' path. The dislocation may then continue to glide, or it may be annihilated with another dislocation of the opposite sign. Either way, the back stress is relieved and the dislocation source can emit another loop. This is the recovery event, the rate of which is controlled by the rate of vacancy diffusion to the climbing dislocation. Many other dislocation models have been proposed (see for example [Garofa.'65]) based upon finding the steady state or minimum creep rate defined at the point when the strain hardening and time softening rates are equal, such that $\varepsilon_{m}^{\prime}=r / h$, where $r$ is the rate of recovery $(d \sigma / d t)$, and $h$ is the hardening rate $(d \sigma / d \varepsilon)$. These models generally predict a power law relation between stress and strain rate, with a stress exponent of around 3-4. This exponent is often referred to as the ' $n$ ' value.

\section{Diffusional Creep}

Diffusional creep is the dominant mechanism under lower stress conditions. Grain boundaries orientated perpendicular to the applied tensile stress direction are considered to be sources of vacancies, and those boundaries parallel to the principal stress (being placed in a state of compressive or zero stress), are considered to be sinks for vacancies. The difference arises because the energy required to create a vacancy at a tensile boundary is lowered, and hence a vacancy concentration builds up across the grain. It is this concentration gradient which is the driving force for vacancy diffusion from the tensile to compressive boundaries, or conversely the diffusion of atoms from compressive to tensile boundaries. The plating of atoms along the tensile boundaries is the mechanism by which strain is accumulated within the metal. When the temperature is high $\left(>0.7 \mathrm{~T}_{\mathrm{m}}\right)$ diffusion occurs predominantly through the lattice [Nabarr.' 48 ], [Herrin.'50], giving a creep rate dependence on grain size (d) of $1 / \mathrm{d}^{2}$. However, when the temperature is lower, diffusion occurs preferentially along the grain boundaries [Coble'63], providing a $1 / \mathrm{d}^{3}$ grain size dependence. Whichever mechanism is dominant at a given time, the stress dependence of the minimum creep rate for diffusional creep is approximately linear (ie. $n=1)$. 


\section{The Effect of Alloying Elements on Creep Deformation}

The addition of alloying elements to a pure metal may increase the creep resistance by solid solution strengthening and precipitation or particle hardening. The minimum creep rate may be decreased by a factor of up to 100 by solid solution strengthening, but more effective reductions are obtained by using a high quantity of precipitates or particles such as are found in modern superalloys. In this case, reductions in the minimum creep rate may be up to a factor of $10^{10}$.

\section{Solid Solution Strengthening}

The addition of elements in solid solution may change the diffusion rates of the parent metal. For example, the addition of carbon to iron increases the mobility of the iron atoms at high temperature, thereby decreasing the creep resistance. In this way both diffusional and dislocation controlled creep mechanisms may be accelerated or decelerated depending on the relative diffusion coefficients with and without solid solution alioying elements. However, the presence of solute atoms may also change the actual dislocation creep mechanisms themselves. As a result of this, two types of single phase alloys have been defined; namely class II (or class M) which show characteristics similar to pure metals, and class I (or class A) which exhibit anomalous creep behaviour [Sherby'67]. Most solid solutions show class M behaviour and therefore have similar dislocation mechanisms to pure metals with an $\mathrm{n}$ value around 4 . Strengthening comes from impeding dislocation glide and recovery processes. Class A alloys often show a change in stress exponent with changing solute concentration. This reflects a change in dislocation mechanism as solute atoms become attracted to dislocations due to size differences with the solvent atoms. The creep rate then becomes controlled by the rate with which a dislocation can move whilst dragging its' solute atmosphere [Weertm. '60].

\section{Precipitation and Particulate Hardening}

Precipitation or particulate hardening provides the greatest contribution to high creep strength in modern alloys. Precipitates may pin the grain boundaries making grain boundary sliding and the generation of vacancies more difficult, thus hindering diffusional creep mechanisms. The most significant strengthening however comes from direct interaction between particles and dislocations. In order for a dislocation to glide it must now overcome these obstacles. Bowing between the particles or shearing of the particles may occur at high stresses, but for lower stress conditions more typical in industrial plant the dislocations must climb around the obstacles [Ansell'59]. Despite the similarity of the climb mechanism in pure metals and particle 
strengthened alloys there is a large anomaly in the $\mathrm{n}$ value, with values in alloys normally much higher than 4. Whilst no mechanistic reason for this has yet been found it is often reconciled in terms of an "internal" stress which is a measure of the resistance of the material to an applied stress. Thus the effective stress in the alloy becomes:

$$
\sigma_{\mathrm{e}}=\sigma_{\mathrm{a}}-\sigma_{\mathrm{i}}
$$

where $\sigma_{\mathrm{e}}$ is the effective stress, $\sigma_{\mathrm{i}}$ is the applied stress and $\sigma_{\mathrm{i}}$ is the internal stress. Experimental measurements of the internal stress have been made using stress dip tests [Davies'73]. Incorporation of these into the effective stress equation above provides new stress exponents around 4 as originally expected.

The major problem with precipitate strengthened alloys is that precipitates have a tendency to coarsen with time. This produces a material with larger but more spaced out precipitates which means that their effectiveness in hindering dislocation glide is reduced. The problem is partially overcome in low alloy steels by relying on a sequence of precipitates, such that as one type of carbide becomes unstable another stable one is forming. In modern superalloys coherent precipitates such as $\mathrm{Ni}_{3}(\mathrm{Al}, \mathrm{Ti})$ in a nickel alloy matrix are used. The coherency between particle and matrix increases stability. Alternatively, insoluble particles such as oxides may be added by powder milling techniques. These form the basis of the Oxide Dispersion Strengthened (ODS) alloys which rely on the extremely low solubility of the particles to overcome coarsening.

\section{Creep Damage Mechanisms}

As creep deformation accumulates, the creep rate will begin to increase during the tertiary phase. This acceleration is due to the gradual build up of damage within the material, which may eventually lead to fracture. Fracture is often classified as ductile or brittle, with transgranular or intergranular damage mechanisms being respectively dominant (Figure 2.6). Four broad categories of damage have been identified [Ashby'84]:

1. Loss of internal section (e.g. cavitation)

2. Loss of external section (e.g. necking)

3. Damage by degradation of the microstructure (e.g. Ostwald ripening)

4. Environmental damage (e.g. Hydrogen Attack) 
Figure 2.6: Broad classification of ductile and brittle creep damage. (from [Ashby'79])

\section{Triple Point Cracking}

Triple point cracking is a type of damage associated with a loss of internal section. Under high stress conditions grain boundary sliding can result in large stress concentrations at grain boundary triple points. When these stress concentrations are high enough, decohesion of the grains can occur and 'Triple point' cracks nucleate. Growth of the crack may then continue along those boundaries situated normal to the applied tensile stress. It has been found that there is a limiting stress concentration, and hence limiting applied stress for triple point cracking to occur. Thus, as the applied stress is decreased a transition in damage mechanism is observed and cavitation becomes predominant.

\section{Cavity Nucleation}

Grain boundary cavities are common in long term creep tests under relatively low stresses. This is a type of loss of internal section, and will be discussed in some detail in the following paragraphs due to their frequent appearance and the similarity to hydrogen attack voids. Cavities are thought to nucleate from grain boundary sliding events as illustrated in Figure 2.7. The necessary high stress concentrations can be created where ledges or steps caused by slip planes intersect the grain boundary and impede sliding. Particles on the boundary may also aid in nucleation by shearing or decohering with the matrix. Nucleation occurs preferentially on those boundaries orientated normal to the applied stress. Demonstration of strain related nucleation has been shown in creep tests on pre-strained material (see for example [Dyson'73]), where increased cavitation and reduced creep lives are often recorded. In addition, cavities may also form at the tips of carbides during tempering of low alloy steels due to thermal effects 
Figure 2.7: Intergramular cavity mucleation processes (from /Goods'83])

[Lopez'83]. In many metals and alloys, stochastic boundary sliding throughout the creep life leads to continuous cavity nucleation [Argon'83],[Dyson'83]. Furthermore, the number of nuclei tend to increase linearly with strain during at least the early stages of the creep life. Under these conditions, deformation induced cavity nucleation, rather than the subsequent growth, may control the fracture time [Goods' 83 ].

\section{Unconstrained Cavity Growth}

Whilst there is reasonable agreement on the mechanisms of cavity nucleation, the growth of grain boundary cavities has been subject to several various theories. It was recognised that grain boundary cavities could grow by the diffusion of vacancies along the boundary. The first model of this process was that of Hull and Rimmer, who treated the boundary as rigid and a perfect source of vacancies [Hull'59]. In this model, similar to diffusional creep, the cavity growth rate was found to be linear with stress, with failure time being inversely proportional to stress. This is generally not the case as a power law is commonly observed for the stress dependence of lifetime. To overcome this, Beere and Speight modified the Hull and Rimmer model to allow the grain boundaries to relax by creep, thereby accommodating the atoms that diffuse into the grain boundary [Beere'78]. A critical diffusive path length existed, beyond which diffusion became difficult and the creep accommodation became important. It was found that the ratio of cavity radius to intercavity spacing determined the growth rate, with smaller spacings giving higher growth rates. 


\section{Surface Diffusion Controlled Cavity Growth}

Further theories suggest that diffusion along the cavity surface may be much slower than along the grain boundary, such that surface diffusion becomes controlling [Chuang'73]. Evidence for this comes from the penny shaped or crack like shaped voids that are sometimes observed, indicative of relatively slow surface diffusion.

\section{Source Controlled Cavity Growth}

One of the largest drawbacks of the above models is the assumption that grain boundaries are perfect sources of vacancies. In reality the presence of precipitates may reduce the ease of vacancy formation on the boundaries [Harris'73]. Vacancies must then be created by dislocation motion and climb at or near to the boundary such that these vacancy creation events may become rate controlling under certain conditions [Beere' 80 ]. This type of mechanism is referred to as 'source controlled cavity growth'.

\section{Constrained Cavity Growth}

Constrained cavity growth models consider that the cavities can only grow at a rate determined by the creep accommodation of the surrounding grains. Dyson was the first to model this phenomenon [Dyson'76]. The model was well accepted as it showed a strain rate dependence of the cavity growth rate, and hence the lifetime. In a review of cavity growth models, Riedel showed that the constrained models give excellent agreement with experimental results on low alloy steels [Riedel'89].

\section{Summary of Cavitation Damage}

In reality, cavities may grow by a number of mechanisms acting independently or in conjunction with each other. The mechanisms that are active at a given time depend upon the stress, temperature and material under study, although it is now generally accepted that a combined diffusion and creep process acts for most conditions of interest.

\section{Loss of External Section}

Damage by loss of external section occurs as the specimen thins due to accumulated strain. As mentioned in the introduction to creep, constant stress tests account for a uniform thinning by reducing the applied load. However, at some point mechanical instability occurs and a neck begins to form. Instability has been suggested to begin just prior to the secondary creep stage. However, for materials with 'normal' $\mathrm{n}$ values of around 5-10, the neck is not thought to 
significantly change the minimum creep rate [Burke'75], although during the later stages of tertiary creep the increased local stress and strain invariably causes failure in this area. Under high temperature conditions $\left(>0.8 \mathrm{~T}_{\mathrm{m}}\right)$ in pure metals and some solid solution alloys dynamic recrystallisation can occur, resulting in the removal of intergranular damage. In this case the specimen may neck to a point at failure. Normally however, some other fracture mechanism intervenes such as intergranular cavity coalescence or ductile transgranular voiding.

\section{Microstructural Degradation}

Degradation of the microstructure is normally related to particle coarsening in precipitate strengthened alloys. For example, in low alloy steels the fine carbides that give the high creep strength will evolve, becoming larger and more spaced out. As particle spacing increases dislocations may move easier through the matrix, thus increasing the creep rate. High temperatures accelerate the carbide evolution [Baker'59], with stress able to accelerate certain transformations [Gope'92].

\section{Environmental Damage}

Environmental damage may occur in many situations. For example oxygen may penetrate a material to form oxides on the grain boundaries which subsequently act as nucleation sites for cavitation. In addition, rapid oxidation of the specimen surface can cause the effective cross section to decrease with time. Hydrogen attack creates methane bubbles within the steel, similar to, and interacting with, cavitation. The effect of hydrogen attack and combined creep loading is the subject of the following section. 


\subsection{Interaction Between Creep and Hydrogen Attack}

Most industrial components working under high temperature and pressure hydrogen will also be exposed to some sort of stress. In many situations, this stress may be a direct result of the high pressures used in the reactor vessels. It is therefore important to understand the role of combined creep and hydrogen attack. From a practical viewpoint this has not always been possible due to the difficulties of designing safe and cost effective testing facilities capable of operating under the necessary high temperature and pressures with such a volatile gas. As a result, most attention in the literature is given to tensile or creep properties of a steel measured after, rather than during, exposure to hydrogen. Nevertheless, some results of stress rupture tests in a hydrogen environment exist, with full creep curves of strain vs. time being rare. These results together with some theoretical considerations will form the basis of this section of the literature review.

\section{Mechanistic Interactions in Combined Creep and Hydrogen Attack}

\section{Void Nucleation Under Combined Creep and Hydrogen Attack}

It was shown previously in equation 2.7 that an applied stress can reduce the critical void radius required for successful nucleation of a methane filled void. Therefore, under a simple tensile stress, the number of pre-existing voids having the critical radius would be higher and hence, the number of nuclei observed in a steel undergoing attack would be expected to be greater, especially on those grain boundaries orientated perpendicular to the applied stress. In addition, a creeping metal may very well nucleate new cavities by grain boundary sliding. Under creep alone these nuclei may become stable or sinter shut. However, if a methane pressure can build up inside of them then the critical radius decreases and more cavities would become stable. Thus nucleation by hydrogen attack is aided by an external stress, and creep cavity nucleation is aided by an internal methane pressure. In this way, the theoretical combined effect of hydrogen attack and creep is to nucleate more voids than the sum of the two processes together. In addition, the decaying nucleation rate due to hydrogen attack found in low alloy steels [Sundar.' 80], may be partially offset by the continuous nucleation process observed under creep [Dyson'83]. 


\section{Void Growth Under Combined Creep and Hydrogen Attack}

Void growth under externally applied stress or internal methane pressure is very similar. In fact, modelling of the hydrogen attack void growth phenomena is usually based around the creep cavitation models discussed previously. Normally some adjustment is made to account for the internal methane pressure, which is often treated as an externally applied remote stress, such that the applied stress, $\sigma_{\mathrm{a}}$, becomes $\sigma_{\mathrm{a}}+\mathrm{P}$, where $\mathrm{P}$ is the internal methane pressure (see section 2.5 for details on hydrogen attack modelling). Thus the driving force for cavity growth is increased and a faster cavity growth rate is observed. Combining the increased growth and nucleation rates results theoretically in significantly reducing the lifetime of steels undergoing combined creep and hydrogen attack.

\section{Results of Creep-Hydrogen Attack Interaction From the Literature}

Those results of combined creep and hydrogen attack known to the author have been summarised in tables 2.1 and 2.2. Not all the results are directly comparable as some of the experiments were conducted on test pieces of non-standard geometry. However, the general trends are described below.

\section{The Effect of Creep-Hydrogen Attack Interaction on Rupture Ductility}

The most common observation in nearly all the results is that the rupture ductility tends to decrease in hydrogen, especially under longer term tests at higher temperatures and pressures. This is accompanied with a change in fracture from transgranular to intergranular. The nucleation, growth and coalescence of methane filled cavities is normally the reason for the change in rupture mode, with more rapid damage occurring in plain carbon steels than in low alloy steels. In fact, those steels containing more than $2.25 \% \mathrm{Cr}-1 \% \mathrm{Mo}$ are difficult to attack under most of the conditions studied. Not only is the methane pressure reduced in low alloy steels, but also the higher creep strength tends to reduce cavity growth rates.

\section{The Effect of Creep-Hydrogen Attack Interaction on Creep Rupture Strength}

The change in rupture strength under combined hydrogen attack and creep is less obvious than the decrease in ductility. For carbon steels, the rupture strength is generally decreased, as would be expected, due to the rapid growth of methane filled cavities. Proof of the importance of methane comes from those experiments on very low carbon steel, which showed no reduction of 


\begin{tabular}{|c|c|c|c|c|c|c|}
\hline \multirow[t]{2}{*}{ Material } & \multicolumn{2}{|c|}{ Test Conditions } & \multirow{2}{*}{$\begin{array}{c}\text { Creep } \\
\text { Rupture } \\
\text { Lifes (hrs) } \\
\end{array}$} & \multirow{2}{*}{$\begin{array}{c}\text { Metallographic } \\
\text { Observations }\end{array}$} & \multirow{2}{*}{$\begin{array}{c}\text { Results of Creep } \\
\text { Properties }\end{array}$} & \multirow[t]{2}{*}{ Reference } \\
\hline & $\begin{array}{l}\text { Temp. } \\
\text { (C) }\end{array}$ & $\begin{array}{l}\text { Press. } \\
(\mathrm{MPa})\end{array}$ & & & & \\
\hline $2.25 \mathrm{Cr}-1 \mathrm{Mo}(\mathrm{Q} \& \mathrm{~T})$ & 454-595 & 13.8 & $50-10000$ & None detailed & Increased rupture life! & Erwin 82 \\
\hline $2.25 \mathrm{Cr}-1 \mathrm{Mo}(\mathrm{Q} \& \mathrm{~T})$ & $420-510$ & 13.8 .20 .7 & $260-5700$ & $\begin{array}{l}\text { Cavitation increasingly apparant } \\
\text { for long times \& high temperatures }\end{array}$ & $\begin{array}{l}\text { No effect on rupture life. } \\
\text { Ductility reduced, especially under long t, high } \mathrm{T}\end{array}$ & Norris 76 \\
\hline $2.25 \mathrm{Cr}-1 \mathrm{Mo}(\mathrm{N} \& \mathrm{~T})$ & 600 & 13.8 & $100-300$ & $\begin{array}{l}\text { Enhanced cavity nucleation and } \\
\text { growth under stress }\end{array}$ & Decrease in rupture life & Wanage 82 \\
\hline $\begin{array}{l}2.25 \mathrm{Cr}-\mathrm{IMo} \\
(\mathrm{A}, \mathrm{AT}, \mathrm{NT})\end{array}$ & $450-550$ & 9.91 & $3-700$ & $\begin{array}{l}\text { Cavitation and mixed mode } \\
\text { fracture apparent only for } \\
\text { highest temperat'ire tests in } \\
\text { hydrogen }\end{array}$ & $\begin{array}{l}\text { Stress rupture strength lowered. with effect greatest } \\
\text { at higher temperatures in annealed material. } \\
\text { Creep ductility lowered only at high temperature } \\
\text { low stress conditions }\end{array}$ & Yokaga. 84 \\
\hline $\begin{array}{c}2.25 \mathrm{Cr}-1 \mathrm{Mo} \\
0.5 \mathrm{Mo}-0.006 \mathrm{~B}\end{array}$ & 540 & 6.2 & $23-160$ & $\begin{array}{l}\text { No fissures in } 2.25 \mathrm{Cr} \\
\text { Some in } 0.5 \mathrm{Mo}-0.006 \mathrm{~B}\end{array}$ & Some reduction in stress rupture strength & Sponse. 66 \\
\hline $\begin{array}{l}2.25 \mathrm{Cr}-1 \mathrm{Mo} \\
\text { and } \mathrm{V} \text { modified } \\
\text { (also weld material) }\end{array}$ & 454 & $14-21$ & $200-50000$ & $\begin{array}{l}\text { Failure occurs in } \mathrm{HAZ} \text { or } \\
\text { weld material in hydrogen for } \\
\text { standard material. No } \mathrm{HAZ} \\
\text { failures for V modified steel. } \\
\text { Cavitated grain boundaries } \\
\text { observed. less in V steels }\end{array}$ & $\begin{array}{l}\text { Some reduction in stress rupture strength. but } \\
\text { stress rupture curve slope remains same. } \\
\text { Effect greatest at high } T \text { and } P \text {. }\end{array}$ & Lundin 94 \\
\hline $\begin{array}{c}2.25 \mathrm{Cr}-1 \mathrm{Mo} \\
3 \mathrm{Cr}-1.5 \mathrm{Mo}(\mathrm{Q} \& \mathrm{~T})\end{array}$ & 600 & 13.8 & $304-1186$ & $\begin{array}{c}\text { Cavitation seen in } 2.25 \mathrm{Cr}-1 \mathrm{Mo} \\
\text { only }\end{array}$ & $\begin{array}{l}2.25 \mathrm{Cr}-1 \mathrm{Mo} \text { loss in both rupture life and ductility. } \\
3 \mathrm{Cr} \text { material more resistant }\end{array}$ & Ruoff 87 \\
\hline $\begin{array}{l}2.25 \mathrm{Cr}-1 \mathrm{Mo}(\mathrm{Q} \& \mathrm{~T}) \\
3 \mathrm{Cr}-1.5 \mathrm{Mo}(\mathrm{Q} \& \mathrm{~T})\end{array}$ & $420-510$ & $13.8-20.7$ & $150-10000$ & $\begin{array}{l}\text { Increased number of grain } \\
\text { boundary voids with hydrogen. } \\
\text { Fracture becomes more intergranular } \\
\text { and brittle with longer times for } \\
2.25 \mathrm{Cr}-1 \mathrm{Mo} \text { only }\end{array}$ & $\begin{array}{l}\text { No effect on rupture lives. } \\
\text { Ductility and reduction of area decreases a lot in } \\
2.25 \mathrm{Cr}-1 \mathrm{Mo} \text { but not in } 3 \mathrm{Cr}-1 \mathrm{Mo} \text {. }\end{array}$ & Ciuffr 82 \\
\hline $\begin{array}{l}\text { Cr-Mo alloy steels } \\
\text { from } 1-9 \% \mathrm{Cr} \\
\text { (A.Q\&T) }\end{array}$ & 593 & 6.2 & $15-700$ & $\begin{array}{l}\text { Fissures near fracture noticed for } \\
\text { annealed materials. }\end{array}$ & $\begin{array}{l}\text { Reduction of stress rupture for annealed materials. } \\
\text { High Cr tempered alloys show increase in rupture } \\
\text { properties, attributed to hydride phase. }\end{array}$ & Mullan.67 \\
\hline
\end{tabular}




\begin{tabular}{|c|c|c|c|c|c|c|}
\hline \multirow[t]{2}{*}{ Material } & \multicolumn{2}{|c|}{ Test Conditions } & \multirow{2}{*}{$\begin{array}{c}\text { Creep } \\
\text { Rupture } \\
\text { Lifes (hrs) }\end{array}$} & \multirow{2}{*}{$\begin{array}{l}\text { Metallographic } \\
\text { Observations }\end{array}$} & \multirow{2}{*}{$\begin{array}{c}\text { Results of Creep } \\
\text { Properties }\end{array}$} & \multirow[t]{2}{*}{ reference } \\
\hline & $\begin{array}{l}\text { Temp. } \\
\text { (C) }\end{array}$ & $\begin{array}{l}\text { Press. } \\
(\mathrm{MPa})\end{array}$ & & & & \\
\hline $\begin{array}{l}\text { SAE1020 mild steel } \\
\text { (N) }\end{array}$ & $425-540$ & $2.7-9.6$ & $1-500$ & $\begin{array}{l}\text { Fissuring and decarburisation } \\
\text { obvious from intermediate tests. } \\
\text { Voids larger and more numerous } \\
\text { in hydrogen. Increase in } \\
\text { intergranular failure with time }\end{array}$ & $\begin{array}{l}\text { Loss of ductility high. Loss in rupture time } \\
\text { increases with increasing time and pressure }\end{array}$ & Allen 62 \\
\hline $\begin{array}{l}\text { Modified mild steel: } \\
1 \% \mathrm{Cr}-0.5 \% \mathrm{Mo} \\
0.5 \% \mathrm{Mo}\end{array}$ & 540 & $2.7-9.6$ & $1-3000$ & $\begin{array}{l}\text { Fissuring and G.B. cavities } \\
\text { obvious from intermediate tests. } \\
\text { Voids larger and more numerous } \\
\text { in hydrogen }\end{array}$ & $\begin{array}{l}\text { Loss of rupture strength and ductility } \\
\text { increases with pressure. For } 1 \% \mathrm{Cr} \text { steel there was } \\
\text { relatively less damage at severe conditions }\end{array}$ & Suther. 65 \\
\hline $\begin{array}{l}\text { low carbon steel } \\
<100 \text { ppm C (A) }\end{array}$ & 500 & 9.9 & $1-1000$ & $\begin{array}{l}\text { Grain boundary voids seen in } \\
\text { hydrogen. Increasing intergranular } \\
\text { fracture with time }\end{array}$ & $\begin{array}{l}\text { Rupture time and ductility decreased. Decrease } \\
\text { more apparant at longer times. Creep curves show } \\
\text { shorter and more rapid secondary creep. }\end{array}$ & Yokoga. 89 \\
\hline $\begin{array}{c}\text { Ferrovac } \mathrm{E} \text { and } \\
\text { hydrogen } \\
\text { Ferrovac } \mathrm{E}<15 \mathrm{ppm} \mathrm{C}\end{array}$ & 540 & 6.2 & $5-500$ & $\begin{array}{l}\text { Voids and fissures in Ferrovac } E \\
\text { only. Also increase in intergranular } \\
\quad \text { fracture }\end{array}$ & $\begin{array}{l}\text { From creep curves extent of secondary creep } \\
\text { is reduced. Effect greatest for longer times and only } \\
\text { noticed on Ferrovac E, not decarburised Ferrovac E }\end{array}$ & Thoma' 66 \\
\hline $\begin{array}{l}10 \text { wrought or cast } \\
\text { alloys inc. alloy } 800 \mathrm{H}\end{array}$ & $705-905$ & 15 & $1-1200$ & no significant effect on fracture & $\begin{array}{l}\text { Reduction in ductility, no obvious change in } \\
\text { rupture strength }\end{array}$ & Bhatta. 85 \\
\hline
\end{tabular}

$\mathrm{Q}=$ quenched, $\mathrm{A}=$ annealed, $\mathrm{N}=$ normalised, $\mathrm{T}=$ tempered 
rupture strength in hydrogen [Thoma'66]. The results for low alloy steels are less conclusive, with some experiments producing increased lifetimes or showing no change at all. Satisfactory explanations for this behaviour are not available.

\section{Results of Multiaxial Stressing under Hydrogen Attack Conditions}

Results of creep tests performed under multiaxial stress in a hydrogen environment are rare. Early tests carried out in Russia investigated the effect of internal hydrogen pressure on the creep rupture of tubular components [Kolgat.'59]. Various metals and alloys ranging from iron to chromium and titanium stabilised stainless steels were tested in the temperature range of 400 to $600^{\circ} \mathrm{C}$. Reductions in the rupture strength were observed for all the materials when compared with control tests pressurised with nitrogen, the effect being greatest at high temperatures and long test times. Up to $75-85 \%$ loss in rupture strength was observed for iron and very low alloy steels, falling to $10-20 \%$ in the highest alloy steels. The reductions in rupture strength were normally accompanied by a decrease in ductility, except in the high alloy steels.

Similar tests on $2.25 \mathrm{Cr}-1 \mathrm{Mo}$ steel showed no effect of hydrogen at $480^{\circ} \mathrm{C}$ and $13 \mathrm{MPa}$ hydrogen for exposure times as long as 2900 hours [Sticha'69]. However, increasing the temperature to $540^{\circ} \mathrm{C}$ lead to a decrease in lifetime of approximately $60 \%$, with the effect being greatest with longer exposures. Nevertheless, no fissures typical of hydrogen attack were noticed, and surface decarburisation was only slight. Welded specimens showed no additional loss of life. Control experiments with helium gas were correlated with uniaxial rupture specimens using the mean diameter hoop stress.

More recently, internally pressurised tubes of alloy $15 \mathrm{Ch} 3 \mathrm{MFA}-\mathrm{A}$ steel (approx. 2.65\% Cr, $0.6 \% \mathrm{Mo}, 0.3 \% \mathrm{~V}$ ) were examined [Karzov'94]. Temperatures of $450^{\circ} \mathrm{C}-550^{\circ} \mathrm{C}$ were used in conjunction with $35-60 \mathrm{MPa}$ gas pressure. Failure times of up to 15000 hours were investigated, with no reduction in rupture strength compared with the control experiments observed for any of the conditions studied.

\section{Hydrogen Attack Assisted Creep Crack Growth}

In the last few years attention has been placed on the phenomena of hydrogen attack assisted creep crack growth (HAACCG). The first results of HAACCG in the literature are from tests on a low carbon steel [Shewmo.'91]. It was found that under conditions of temperature and pressure where normal attack (ie. cavitation) was not found, it was possible to accelerate the 
rate of crack growth. Further results on $2.25 \mathrm{Cr}-1 \mathrm{Mo}$ steel showed the same trend with no conventional damage being found away from the crack [Chen'95]. These investigations cite the stress intensity at the crack tip to be the controlling factor, with the high stress concentrations causing localised hydrogen attack in the form of methane bubbles just ahead of the crack.

Separate work conducted on $2.25 \mathrm{Cr}$-1 Mo steel under multiaxial stress conditions at higher temperatures showed the same basic trend, although a different form of the crack tip loading parameter was found to correlate the results [Looney'94].

Whilst little work has been carried out in this area, it is of great interest as it shows that a crack can propagate rapidly in the absence of normally recognised hydrogen attack damage. Component integrity examinations must therefore take this into consideration. 


\subsection{The Alloy 2.25Cr-1Mo}

\section{Introduction To The Alloy 2.25Cr-1Mo}

$2.25 \mathrm{Cr}-1 \mathrm{Mo}$ is a very widely used alloy, especially in applications such as pressure vessels and piping in power plants and in the chemical and oil processing industries. The good high temperature properties, corrosion resistance, fabricality and low price make it particularly attractive. This steel evolved from the need to use higher operating temperatures in industrial plant. Early empirically derived alloy additions to C-Mn steel eventually lead to the development of $2.25 \mathrm{Cr}-1 \mathrm{Mo}$ steel, which was included in the ASTM specifications in the annealed form as early as 1952 . Since then the effects of different heat treatments and minor alloy additions have been thoroughly investigated, and a good understanding of the interrelation between the wide-ranging possible microstructures and properties has arisen.

\section{Microstructure-Property Relations in $2.25 \mathrm{Cr}-1 \mathrm{Mo}$}

The microstructures found in $2.25 \mathrm{Cr}-1 \mathrm{Mo}$ depend critically on the heat treatment given. The following will describe the main commercial heat treatments performed, their effect on the microstructure, and, subsequently, on the properties of the material. In addition, the effect of minor compositional changes will be examined.

\section{Heat Treatments of $2.25 \mathrm{Cr}-1 \mathrm{Mo}$ Steel}

The common heat treatments, as governed by ASTM standard A387 [ASTM387], include annealing, normalising and tempering, and quenching and tempering.

Annealing involves an austenitising treatment at a temperature of between approximately 910$940^{\circ} \mathrm{C}$. At this temperature $\mathrm{Cr}$ and Mo are completely dissolved in the austenite. The steel is then cooled slowly from the austenitising temperature in a furnace, or is held isothermally at around $700^{\circ} \mathrm{C}$ for some time during cooling. This procedure produces a near equilibrium structure consisting predominantly of ferrite and pearlite, with some bainite possible under slightly faster cooling conditions.

Normalising entails austenitising at a similar temperature to that used in annealing. However, a faster 'air cool' is used, the rate of which depends partly on the size and geometry of the steel casting. The microstructure is dependant on the cooling rate, with slow rates producing a mainly ferritic structure, and fast rates giving chiefly bainitic and even martensitic structures. Steels in 
this condition are normally given a tempering treatment to increase toughness. The effect of tempering is discussed in more detail later.

Quenching involves rapidly cooling the steel from the austenitising temperature by immersing in oil. The high cooling rates yield a bainite-martensite mixture. Tempering is usually necessary.

Tempering is usually carried out after normalising and quenching operations. It improves the toughness of the material, albeit at a cost to the strength. Typical tempering is carried out at temperatures above $675^{\circ} \mathrm{C}$ and normally below about $750^{\circ} \mathrm{C}$, for several hours, the times increasing at lower temperatures. During tempering the precipitation and evolution of various carbides may occur, changing the properties of the steel. The most recognised work in the field was that conducted by Baker and Nutting whose results on the evolution of carbides are summarised in the schematic below [Baker'59]:

1) Quenched 2.25Cr-1 Mo:

$$
\begin{aligned}
& \longrightarrow \underset{\downarrow}{\mathrm{Cr}_{7} \mathrm{C}_{3}} \\
& \varepsilon \text {-carbide } \rightarrow \text { cementite } \rightarrow+\longrightarrow \mathrm{M}_{23} \mathrm{C}_{6} \rightarrow \mathrm{M}_{6} \mathrm{C} \\
& \mathrm{Mo}_{2} \mathrm{C}
\end{aligned}
$$

2) Normalised $2.25 \mathrm{Cr}-1 \mathrm{Mo}$ :

$\sim$ Bainitic areas:

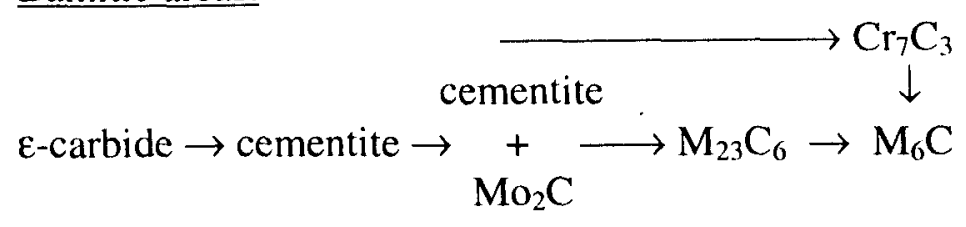

$\sim$ Ferritic areas:

$\mathrm{M}_{2} \mathrm{X} \longrightarrow \mathrm{M}_{6} \mathrm{C}$

The behaviour of the quenched material and the bainitic material in the normalised steel is very similar. It has been suggested that $\mathrm{Mo}_{2} \mathrm{C}$ is more accurately referred to as $\mathrm{M}_{2} \mathrm{X}$, due to the discovery of other elements within the carbide [Murphy'71]. This carbide nucleates rapidly in ferritic steels, and only after long times does it give way to $M_{0} C$. In the bainitic steel $M_{2} X$ nucleates from cementite, but soon transforms to the higher order carbides which eventually end up as the equilibrium carbide $\mathrm{M}_{6} \mathrm{C}$. 


\section{The Effect of Heat Treatment and Microstructure on Creep Properties}

Despite the higher room temperature strength of the quenched steels, it is generally found that the normalising treatment produces more creep resistant material at high temperatures, with the transition being identified at about $500^{\circ} \mathrm{C}$ [Baker' 59]. This is related to the stability of the fine $\mathrm{M}_{2} \mathrm{X}$ precipitates in ferrite, which are the most effective carbides in conferring high creep strength. Thus in the normalised steels containing more ferrite, the stability of the $M_{2} X$ over long times provides lasting strength. On the other hand, the relatively rapid transformation to $\mathrm{M}_{23} \mathrm{C}_{6}$ and $\mathrm{M}_{6} \mathrm{C}$ and subsequent agglomeration in the quenched steel means that creep strength is reduced over time. Furthermore, annealed $2.25 \mathrm{Cr}-1 \mathrm{Mo}$ may have similar creep strength under certain conditions, despite the inherent room temperature softness [Murphy'71]. At very long times $\left(\approx 10^{5}\right.$ hours), all the heats would be expected to be similar due to the formation of a near equilibrium structure.

\section{Compositional Effects in $2.25 \mathrm{Cr}-1 \mathrm{Mo}$}

There are several elements that may be found within a $2.25 \mathrm{Cr}-1 \mathrm{Mo}$ steel, the effects of which may be both beneficial or detrimental. The allowable limits of the different elements can be found in the ASTM standard [ASTM387].

The basic elements of chromium and molybdenum are responsible for the creep strength and oxidation resistance of the alloy. Molybdenum is particularly good at conferring high creep strength by both solid solution and precipitation hardening, with the greatest effect occurring at a concentration of about $1 \%$ [Argent'70]. The $\mathrm{Mo}_{2} \mathrm{C}$ carbide is widely considered the most effective in contributing to this strength. Chromium however may reduce the creep strength by dissolving in the $\mathrm{Mo}_{2} \mathrm{C}$, causing overaging, whilst higher amounts of chromium may lake carbon away from stronger carbide formers such as molybdenum and vanadium [Woodhe.'65], [Stone'65]. These problems (not to mention the additional cost) must be weighed against the advantages of increased oxidation and hydrogen attack resistance [Chung'82], [Wada'82], and improved microstructural stability. The mixture of $2.25 \% \mathrm{Cr}$ and $1 \% \mathrm{Mo}$ gives a good balance between the above factors.

Increasing the carbon content plays a large role in augmenting the strength, hardness and creep properties of the steel, with an optimum creep strength being found between $0.1-0.2 \%$ [Woodhe.'65]. In addition, the amount of carbon influences the ratio of ferrite to bainite that 
forms during heat treatment [Kleuh 74]. Detrimental effects of excess carbon include poor weldability and low toughness.

Manganese is a commonly found element in many steels. It is essential for good weldability in that it assists deoxidisation of the weld metal and prevents hot cracking. However, high manganese contents may reduce the creep strength and increase the susceptibility to temper embrittlement [Sachs'70]. Optimum manganese levels are therefore a compromise, and have been suggested to lie between $0.7-0.85 \%$ [Okumur.' 76 ].

Titanium, vanadium and niobium are carbide formers and therefore increase the creep strength of 2.25Cr-1Mo by forming fine precipitates such as $\mathrm{V}_{4} \mathrm{C}_{3}$ and $\mathrm{NbC}$ [Woodhe.'67], [Argent'70]. These precipitates tend to coarsen with time and lose their effectiveness, albeit at a slower rate than the normal Mo and $\mathrm{Cr}$ carbides. Excess vanadium and niobium can decrease weldability [Tagawa'84].

Silicon is often included in $2.25 \mathrm{Cr}-1$ Mo to improve weldability and strength. However, excess can promote temper embrittlement and reduces the notch ductility of weld material [Kanaza.'77].

Various elements are known to have a detrimental effect. Aluminium and nickel decrease the creep strength whilst sulphur tends to segregate, thereby reducing grain boundary and carbidematrix interfacial strengths [Kanaza.'77], [Masuya.'90]. Phosphorous, antimony, arsenic and tin have may all promote temper embrittlement, however tin has little effect on the creep strength [Hopkin'62].

\section{Creep Behaviour of $2.25 \mathrm{Cr}-1 \mathrm{Mo}$ Steel}

\section{Reference Data for Creep in $2.25 \mathrm{Cr}$-1Mo Steel}

The high usage of $2.25 \mathrm{Cr}$-1Mo steel in industrial applications has lead to a large number of creep studies being carried out. Much of the work was involved in gauging the effects of minor alloy additions on the creep properties as mentioned above. Further work has been performed under varying conditions chosen to match a particular industrial situation. In order to provide a central database, the International Standards Organisation has produced stress rupture curves for two different heat treatments of $2.25 \mathrm{Cr}-1$ Mo [ISO' 81 ]. The ISO curve for a tempering treatment 


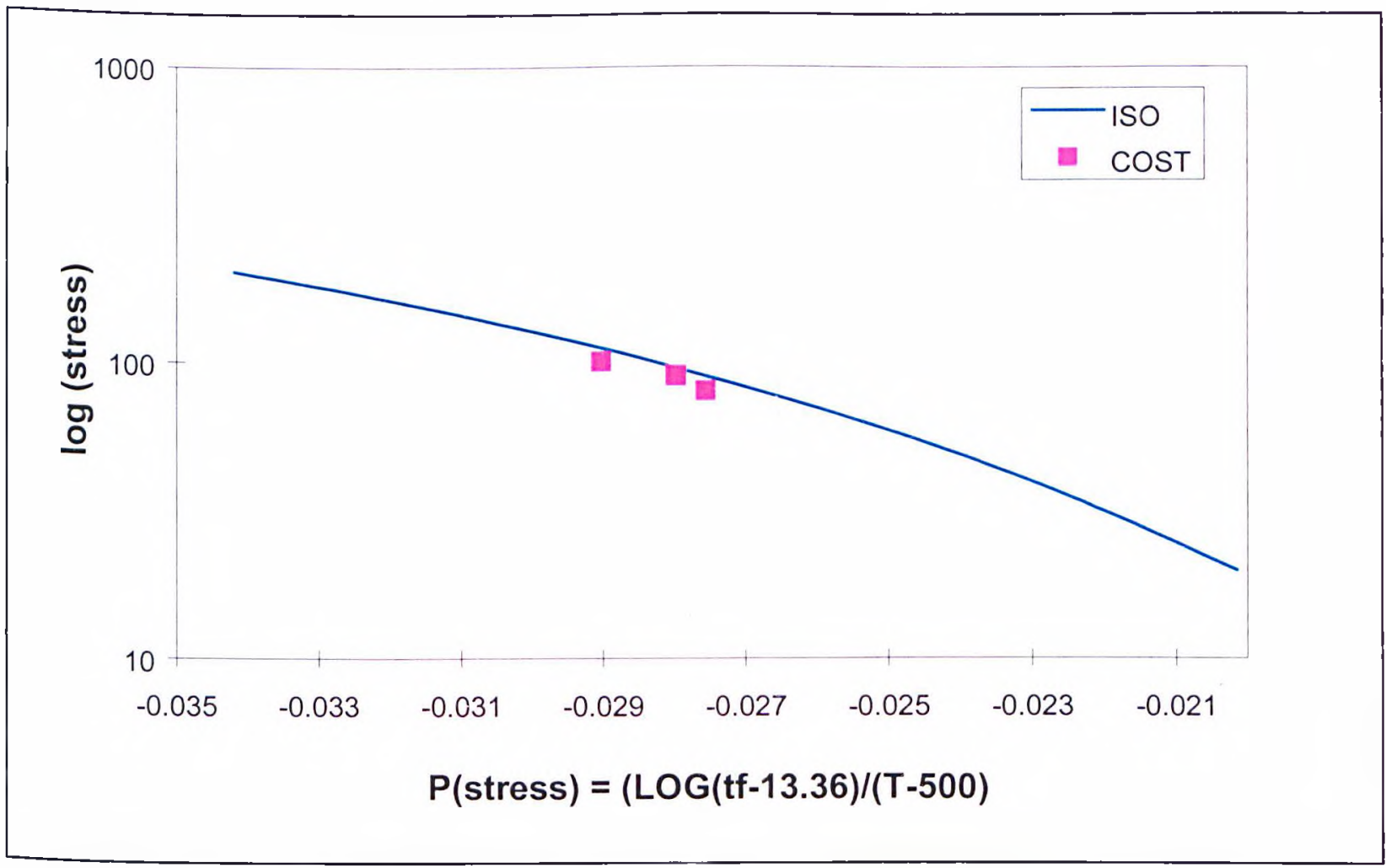

Figure 2.8: ISO stress rupture plot for 2.25Cr-1Mo steel at $600^{\prime \prime} \mathrm{C}$ given a tempering treatment less than $750^{\circ} \mathrm{C}$. Also shown are COST data derived at JRC Petten

at less than $750^{\circ} \mathrm{C}$ is plotted together with some data determined at the JRC Petten [COST'94], in Figure 2.8.

It should be noted when comparing creep databases compiled under different testing conditions, that the mechanisms of creep deformation and fracture may vary, and thus extrapolation models between one data-set and another may be inaccurate. To help overcome this, creep deformation and fracture mechanism maps which give the dominant mechanisms under given conditions of stress, time and temperature can be used (see for example Figure 2.9) [Fields'80].

\section{Creep Cavitation in $2.25 \mathrm{Cr}-1 \mathrm{Mo}$}

As mentioned previously, it is possible for an alloy to fail due to nucleation and growth of grain boundary cavities. It can be scen from the fracture mechanism map (Figure. 2.9), that cavitation occurs preferentially under low stresses, high temperatures and long times. These are the typical conditions found in many industrial plants. In addition, hydrogen attack damage normally takes the form of grain boundary cavities, and hence an understanding of cavitation in $2.25 \mathrm{Cr}-1 \mathrm{Mo}$ is important. 
Figure 2.9: Fracture mechanism map for annealed 2.25Cr-1Mo steel [Fields'80]

Cavity nucleation in bainitic $2.25 \mathrm{Cr}-1 \mathrm{Mo}$ steel has been shown to be proportional to the macroscopic strain under low to intermediate stresses [Cane'79]. The interrelationship is caused by the dependence of nucleation on grain boundary sliding events, which are in turn controlled by the strain in the matrix. It was also found that the majority of the cavities nucleate within the early stages of the creep life on prior austenite grain boundaries and at grain boundary carbides. One theory for the presence of cavities at grain boundary carbides was proposed by Lopez and Shewmon [Lopez'83]. It was suggested that carbide growth during tempering occurs faster than the matrix atoms can diffuse away, thereby setting up local stresses which are high enough to nucleate small voids. These voids may then become enlarged by grain boundary sliding or by an internal methane pressure in the case of hydrogen attack.

Cavity growth in $2.25 \mathrm{Cr}-1$ Mo occurs by a number of mechanisms that depend upon the applied stress and cavity size. A cavity growth mechanism map has been proposed which shows the various controlling mechanisms based upon stress and cavity growth rate [Cane'81]. Under low stresses, initial cavity growth occurs by a source controlled diffusion mechanism, whereas higher stresses allow unconstrained diffusional growth. As the cavity becomes larger, and 
growth rates increase continuum plastic creep flow takes over. For large or fast growing cavities, creep constrained growth takes place. Moreover, under conditions of high stress triaxiality, continuum growth is favoured, leading to lower life and ductility.

It is important to be aware that because of the conditions under which cavitation is dominant, it is sometimes difficult to simulate the industrial damage in laboratory creep experiments conducted under short times.

\section{Hydrogen Attack in $2.25 \mathrm{Cr}-1 \mathrm{Mo}$ Steel}

\section{Industrial Occurrences of Hydrogen Attack in $2.25 \mathrm{Cr}$ - $1 \mathrm{Mo}$}

At the current time there are few examples of hydrogen attack under industrial conditions in this steel. One report states that a $2.25 \mathrm{Cr}-1$ Mo pipeline showed no signs of hydrogen attack after 135000 hours service at $530^{\circ} \mathrm{C}$ with a hydrogen pressure of $3.5 \mathrm{MPa}$ [Bina'96]. The API Nelson curves, which give the safe operating regimes of temperature and pressure for various steels, have only a few data points for $2.25 \mathrm{Cr}$-1Mo [API'97]. Of these, one result shows only surface decarburisation, another showed no attack after 15 years ammonia service at approximately $12 \mathrm{MPa} \mathrm{H}_{2}$ and $460^{\circ} \mathrm{C}$, and only the third set of results shows any internal hydrogen attack. In this particular case, attack occurred in a mixing tee of a hydrogen processing unit operating at about $360^{\circ} \mathrm{C}$ and $11 \mathrm{MPa} \mathrm{H}_{2}$ pressure for more than 20 years. These conditions are within the "safe" region of the Nelson diagram. However, in a nearby part of the vessel no attack was noticed despite having a higher than average temperature. It is rhought that local thermal stresses accelerated the rate of attack in the damaged area. The Nelson curves for $2.25 \mathrm{Cr}-1 \mathrm{Mo}$ steel show its superior resistance to hydrogen attack compared with carbon manganese and $0.5 \% \mathrm{Mo}$ steels. However, no account is made on the diagram for the effect of stress, heat treatment or welds. The following paragraphs aim to show the effect of these parameters.

\section{Hydrogen Attack Under Applied Stress in 2.25Cr-1Mo}

Results of combined hydrogen attack and creep studies in $2.25 \mathrm{Cr}-1 \mathrm{Mo}$ steel have already been presented in table 2.1. It can be seen that in general an applied stress accelerates the rate of damage accumulation compared with hydrogen attack alone. A discussion of the mechanistic explanations of this effect can be found in section 2.3 of this literature review. 


\section{The Effect of Heat Treatment on Hydrogen Attack in $2.25 \mathrm{Cr}$-IMo}

Heat treatments change the type of carbide found in the steel. Thus those heat treatments which produce the most stable carbides would be expected to be more resistant to hydrogen attack. However, the same heat treatments that produce stable carbides are normally those that produce the lowest creep strength, and therefore a balance must be met between these needs.

The effect of tempering on the hydrogen attack resistance of a bainitic quenched and tempered 2.25Cr-1Mo steel was investigated by Parthasarathy and Shewmon [Partha. '84]. It was found that increasing the tempering time reduced the carbon activity of the steel by the formation of stable carbides, which in turn reduced the methane pressure and hence the hydrogen attack cavitation. Excessive tempering beyond commercial practice did not eliminate hydrogen attack due to the slow diffusion of chromium that is necessary in forming the most stabie carbides. However, high temperature tempering treatments followed by fast cooling may be given to service exposed 2.25C-1 Mo to return the tensile strength to its former value before ageing occurred. One of the effects of this is to increase the amount of free carbon in the matrix [Wada'83]. Whilst the author is unaware of any effect of this on the hydrogen attack of $2.25 \mathrm{Cr}$ 1 Mo steel, it has been postulated that the increase in hydrogen attack with tempering found in a C-0.5Mo steel was due to the amount of free carbon in solid solution [Ishigu.'94]. In addition, the cavity nucleation mechanism proposed by Lopez and Shewmon [Lopez' 83 ] would suggest that the number of nucleation sites increases with increasing tempering.

A comparison of quenched and tempered and normalised and tempered $2.25 \mathrm{Cr}-1 \mathrm{Mo}$ showed that the normalised and tempered material exhibited cavity growth rates up to 20 times faster than the quenched and tempered batch [Shewmo.'82]. No explanation was given for this behaviour, although the more rapid stabilisation of carbides during tempering in the quenched material may be a reason. In a study of long term creep under hydrogen attack it was found that annealed material was attacked more than normalised and tempered, which was in turn attacked more than an annealed and tempered batch [Yokoga.' 84 ]. Again no explanation was offered.

\section{Hydrogen Attack in $2.25 \mathrm{Cr}$-1Mo Welds:}

It is well established that hydrogen attack is generally more severe in welds than in base metals, with a greater density of methane induced bubbles being observed (see for example [Partha.' 85$]$ ]): Welds are particularly susceptible as they contain relatively unstable carbides and may be under significant residual stresses. Investigations have shown that hydrogen attack 
resistance can be returned close to that of the base metal by a prolonged post weld heat treatment, and by minimising the weld heat input [Mitsuo' 82]. Despite the potential problems of hydrogen attack in weldments, it is first necessary to understand the problems in base material, and thus further discussion of welds will not be included here.

\section{The Effect of Minor Alloy Additions on Hydrogen Attack in $2.25 \mathrm{Cr}-1 \mathrm{Mo}$}

Alloying elements in $2.25 \mathrm{Cr}-1 \mathrm{Mo}$ steel generally increase the hydrogen attack resistance either by reducing the carbon activity or by increasing the creep strength, making bubble growth more difficult.

Phosphorous and arsenic have been shown to improve the hydrogen attack resistance of $2.25 \mathrm{Cr}$ 1 Mo steel, whilst tin and antimony decrease it [Sakai'81]. Phosphorous and arsenic are believed to segregate to cavity surfaces where they poison the methane reaction. This effect can be offset by the addition of $\mathrm{Sn}$ and $\mathrm{Sb}$, although the mechanism is not clear. Silicon has been shown to promote bubble formation by increasing the ratio of $\mathrm{Fe}$ to $\mathrm{Cr}$ found in the $\mathrm{M}_{23} \mathrm{C}_{6}$ type carbides, thereby increasing the carbon activity and allowing higher methane pressures [Shimom.' 85 ].

Titanium and vanadium have been cited as beneficial for hydrogen attack resistance, either by forming stable carbides or accelerating the evolution of more stable carbides during tempering [Su'84]. In addition, the increased creep strength may provide resistance against cavity growth. Vanadium acts as a trapping site for hydrogen, thereby slowing down the diffusion rate and increasing the solubility [Brouwe.'92]. In this way, the risk of low temperature hydrogen embrittlement during vessel shut down is reduced, and this, combined with the high creep strength, means that vanadium-modified steels are currently being heavily investigated for use in hydroprocessing reactors. Niobium has been found to deteriorate the resistance to attack, despite the beneficial creep properties [Tagawa'84]

\section{Bubble Nucleation in $2.25 \mathrm{Cr}-1 \mathrm{Mo}$}

The results of several investigations of bubble number densities are shown in table 2.3. Direct comparison of the results is difficult due to the various conditions employed and the small number of results. Kinetic studies lead to the conclusion that nucleation in $2.25 \mathrm{Cr}-1 \mathrm{Mo}$ steel is based around pre-existing voids, and that no new voids nucleate due to the internal methane pressure being so low [Sundar.' 80 ]. The only results of active nucleation in $2.25 \mathrm{Cr}$ - 1 Mo are 
those of Thygeson and Molstad, who were able to nucleate numerous grain boundary cavities by exposure to $100 \mathrm{MPa} \mathrm{H}_{2}$ at $500^{\circ} \mathrm{C}$ [Thyges.'64].

\begin{tabular}{|c|c|c|c|c|c|}
\hline $\begin{array}{l}\text { Material } \\
\text { Condition }\end{array}$ & $\begin{array}{l}\text { Temp. } \\
\left({ }^{\circ} \mathrm{C}\right)\end{array}$ & $\begin{array}{c}\mathrm{H}_{2} \text { Pressure } \\
(\mathrm{MPa})\end{array}$ & $\begin{array}{l}\text { Exposure } \\
\text { Time } \\
\text { (hours) } \\
\end{array}$ & 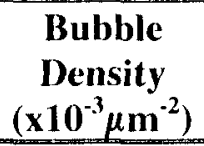 & Ref. \\
\hline $\begin{array}{l}\text { - base metal } \\
\text { - HAZ }\end{array}$ & 600 & 24.3 & 1000 & $\begin{array}{l}6.4 \\
104\end{array}$ & |Sakai'80| \\
\hline $\begin{array}{l}-+0.016 \% \mathrm{P} \\
-+0.016 \% \mathrm{Sn}\end{array}$ & 570 & 31.4 & 500 & $\begin{array}{l}11 \\
80 \\
\end{array}$ & |Sakai'81] \\
\hline $\begin{array}{l}\text { - Q\&T under creep } \\
\text { stress }\end{array}$ & 510 & 20.7 & $\begin{array}{c}\text { variable } \\
(\approx 300-5000)\end{array}$ & $25-30$ & [Ciuffr.' 82 ] \\
\hline $\begin{array}{l}- \text { base metal (A-387) } \\
-+0.25 \mathrm{~V}-\mathrm{Ti}-\mathrm{B}(\text { low } \mathrm{Si})\end{array}$ & 600 & 13.8 & Up to 1440 & $\begin{array}{l}40 \\
<5\end{array}$ & [Su'84] \\
\hline $\begin{array}{l}\text { - base metal } \\
\text { - SMAW-weld metal } \\
\text { - SAW-weld metal }\end{array}$ & 550 & 20.3 & 2500 & $\begin{array}{c}2 \\
10 \\
3 \\
\end{array}$ & [Partha.'85] \\
\hline
\end{tabular}

Table 2.3: Void number density measurements in 2.25Cr-1Mo steel taken from the literature. 


\subsection{Modelling of Hydrogen Attack}

The following section will discuss the development of models of hydrogen attack. First, it will be shown that there is a need for such modelling. Thereafter, a description of several models will be given in chronological order to show the evolution of the modelling approach. Models with and without an externally applied stress have been considered, although nearly all models are based around the creep cavity growth modelling approaches discussed in section 2.2.

\section{The Need for Hydrogen Attack Modelling}

Traditionally, design of components for service in hot hydrogen environments is based upon the empirical Nelson Curves (figure 2.10)[API'97]. These curves give the safe operating temperatures and hydrogen pressures for carbon, Mo and $\mathrm{Cr}$-Mo steels by delineating the pressure and temperature regimes where previous in-service failures have occurred, and those regimes assumed as "safe". However, the reliability of the curves is questionable, as can be well demonstrated for the case of $0.5 \% \mathrm{Mo}$ steel: after more than 20 years of service experience with the $0.5 \%$ Mo steels, the Nelson line has been lowered to that of carbon steel due to failures below the original "safe" $0.5 \%$ Mo line. Nevertheless, there is still a large amount of $0.5 \% \mathrm{Mo}$ equipment that has been functioning satisfactorily for many years in conditions above those of the carbon steel line in the Nelson diagram [VdBurg'96]. Reasons for this include the effect of stress and heat treatment on the resistivity of steels to hydrogen attack; neither of which are accounted for in any detail on the Nelson curves. In addition, the curves are of little use when a new steel such as $2.25 \mathrm{Cr}-1 \mathrm{Mo}-\mathrm{V}$ or $3 \mathrm{Cr}-1 \mathrm{Mo}-\mathrm{V}$ is introduced. It is obvious that improved lifetime predictions of pressure vessel steels are required.

\section{Hydrogen Attack Modelling in the Absence of External Stress}

\section{Early Non Mechanistic Modelling}

One of the earliest models was proposed by Weiner in 1961 [Weiner'61]. His empirically derived equation for the extent of the incubation time in carbon steel is given below:

$$
\mathrm{t}_{\mathrm{i}}=\mathrm{CP}_{\mathrm{H}_{2}}^{-3} \exp \left(\frac{14600}{\mathrm{RT}}\right)
$$


where: $t_{i}$ is incubation time, $P_{H}$, is hydrogen pressure, $R$ is the universal gas constant in cal $/ \mathrm{mol}$, and $\mathrm{C}$ is a constant.

Whilst reasonable agreement with experimental data was achieved at high temperatures, the model rapidly diverges from reality at lower temperatures [Shewmo.'76]. An alternative approach was to use the linear growth rate relations shown in equation (2.8) as derived from experimental dialatometric tests. Assuming that failure occurs relatively shortiy after the onset of the rapid attack stage, and that a critical strain can be found at which incubation ends and rapid attack starts, then predictions can be made of the failure time for given temperature and pressure conditions [Sundar.' 80 ].

To overcome the uncertainties of these rather vague empirical models, several researchers have attempted to define more meaningful mechanistic models. Their work is described in the following paragraphs.

\section{Mechanistic Modelling of Hydrogen Attack in the Absence of an External Stress}

Perhaps the first attempt to mechanistically model the hydrogen attack phenomena was by Shewmon in the mid 70's [Shewmo.'76]. The diffusional creep cavity growth model of Raj and Ashby [Raj'75] was modified by replacing the external stress with the internal methane pressure. It was found that under low temperatures, the cavity growth rate was determined by the carbon availability for the methane reaction, whereas at higher temperatures the diffusional growth of the cavity became rate controlling. Nucleation rates were considered (something often ignored in later models), so that the bubble density was dependant on the carbon activity, since a very high methane pressure was required for active nucleation. It was also shown that hydrogen availability was not a rate-controlling factor due to its high diffusion rate. Whilst this work set the mould for future models, it was only able to rationalise the incubation times when a high density of voids was nucleated.

The next advances in modelling came in 1978 when Sagues, Hall and Wiedersich (SHW) considered cavity growth to be a combination of diffusional and creep processes [Sagues'78]. For the diffusional growth, the corrected Raj and Ashby equations were used [Raj'77], whilst the creep cavity growth was modelled by adapting the work of Finnie and Heller with the internal methane pressure [Finnie'59]. No synergistic effect between combined creep and diffusional growth was considered, neither was the methane pressure calculated with an equation of state that takes into account the fugacity-pressure relationship. However, it was 
predicted that creep growth was important under high pressure, low temperature conditions, whereas diffusional growth only became important at higher temperatures, and in smaller voids.

A major advance was made in 1982 when Shih and Johnson modified the SHW model to include a failure criterion [Shih'82]. Failure was said to occur when the voids grew so large that the ratio of void radius to inter-void spacing was about 0.15 . At this point the individual cavities were assumed to rapidly grow and coalesce, causing massive microcracking. The material constants for $\alpha$ iron were used in the creep controlled growth equations. This was intended to account for the decarburisation that takes place in the immediate vicinity of a methane filled void. Importantly, a transitional radius was defined, above which growth occurs by creep, and below which diffusion is dominant. The prediction was backed up with experimental evidence [McKimp.' 81 ]. The transition radius increases with increasing temperature and with decreasing pressure. Failure time predictions gave reasonable agreement with the Nelson curves, and were relatively insensitive to initial void size, but strongly dependent on void density. However, a fugacity relationship was still missing from this model, leading to uncertainties in the validity of certain conclusions regarding the lack of equilibrium methane pressure in the voids.

Concurrently, Sundararajan and Shewmon [Sundar.81] greatly expanded the original mode! of Shewmon. For the first time, a surface diffusion controlled cavity growth mechanism was considered, following the creep cavity work of Chuang and Rice [Chuang'79]. In addition, constrained cavity growth was thought to be dominant unless each grain boundary facet contained at least one growing bubble. Combined with a more accurate approximation for the methane pressure, reasonable agreement between predicted and experimental kinetic data was achieved, with bubble growth mechanism maps being produced.

In 1982 Odette and Vagarali published their approximate, yet accurate calculation for the methane pressure/fugacity relationship [Odette'82]. This would allow future models to have a much more accurate prediction of the internal methane pressure. That same year, Odette suggested an alternative constrained cavity growth model [Odette'82b]. However, a different balance of forces was used compared with previous authors, and the results were found to be extremely sensitive to the material parameters, especially the fraction of cavitated boundaries, the creep law, and the carbon activity.

Three years later, Parthasarathy developed the latest model of Sundararajan and Shewmon, arguing that enough grain boundary cavities were cavitated in both $2.25 \mathrm{Cr}$ - 1 Mo steel and 
carbon steel to ensure constraint free growth [Partha.' $85 \mathrm{~b}$ ]. Parthasarathy concludes by stating that the main reason for the increase in the resistance of $2.25 \mathrm{Cr}-1 \mathrm{Mo}$ steel to hydrogen attack is the lower bubble density that nucleates.

\section{Mechanistic Modelling of Hydrogen Attack Under an Applied Stress}

The first model to consider the combined effect creep stress and an internal methane pressure was that of Shewmon in 1987 [Shewmo.' 87]. Following Edwards and Ashby [Edward'79], the externally applied stress was considered to promote cavity growth by combined diffusive and local creep mechanisms in addition to the internal methane pressure. The resulting predicted cavity growth rates compared with the case of hydrogen attack alone were greatly increased..It was postulated that those steels with greater creep strength would also have the best resistance to hydrogen attack due to the increased resistance to local creep cavity growth mechanisms. However, the effect of multiaxial stress states was not considered.

The most recent and complete modelling known to the author is that of Van der Burg et al.. In 1995 a model was published which calculated the creep void growth rate in $2.25 \mathrm{Cr}-1 \mathrm{Mo}$ steel by both creep and diffusional mechanisms under high stress triaxialities [VdGies.'95]. It was argued that the presence of an internal methane pressure would exert a highly triaxial stress state on the surrounding material, and therefore previous void growth equations needed to be modified to account for virtually all stress triaxialities. This model was then adapted to account for the internal methane pressure [VdBurg'96]. Whilst both the surface energy and surface tension of the voids were considered, it was assumed that surface diffusion was always fast enough to maintain a semi-equilibrium cavity shape. This was in contrast to some previous models which had suggested surface diffusion to be a major controlling factor [Sundar.'81]. Void nucleation was thought to be present at pre-existing voids found at grain boundary carbides, and thus there was a negligible nucleation time with no new voids later forming. Accurate calculations of the methane pressure were made for the various carbides in the steel (except Mo carbides for which the thermodynamic constants are not defined in the literature), with the methane reaction considered to take place directly on the carbide surface. A failure criterion was included such that calculations of the failure time were predicted for each of the different methane pressures associated with the various carbide types in the steel. It was shown that the effect of stress and the stress triaxiality were extremely important parameters, with orders of magnitude difference in failure time possible under different stress states. 
Further publications applied the void growth equations derived above to situations where non uniform cavitation occurs [VdBurg'94], [VdBurg'96b]. Differences in carbide stability were considered along a grain boundary, leading to non-uniform methane pressure distribution. Two extremes of behaviour were predicted. In the first, creep of the matrix was rapid and lead to little constraint on growth. Bubbles which contained a higher than average pressure were free to grow faster, and thus failure time was controlled by the least stable carbide. In the other extreme, diffusion was relatively fast, and a rigid type of constrained deformation took place. In this case, the average methane pressure in a grain boundary controlled the failure time. Analytical expressions were derived to predict which of the two extremes was dominant under given conditions.

Continued work on this model up through the length scales has lead to the development of a more macroscopic damage relation in 2 and 3 dimensions, which has been applied to the case of the prediction of damage in a pressure vessel wall under non uniform temperature [VdBurg'97]. These predictions, as well as some of the model assumptions have yet been verified with experimental tests.

\section{Disadvantages of Mechanistic Modelling}

Despite the insight that mechanistic modelling gives into the details of the hydrogen attack process, it relies heavily on materials parameters derived from experiment. Thus the accuracy of the model is only as accurate as the experiments. Certain assumptions must also be made. For example, in the above model the methane reaction is assumed to take place on the carbides, nucleation is assumed to be instantaneous, and surface diffusion is not rate controlling. Often the effects of these assumptions is calculated to be negligible, but until further experimental work can verify these assumptions ihere will remain some doubt as to the validity of the models. 


\subsection{Empirical and Phenomenological Creep Modelling}

Whilst the previous paragraphs outlined the mechanistic models of hydrogen attack based on creep cavity growth formulations, it is common to model the creep behaviour of an alloy with more general, macroscopic models. As yet, none of these models have been well defined for the case of hydrogen attack, although the empiricism present should not pose any great difficulty in adding one or more hydrogen effect parameters (assuming that the experimental conditions can be obtained). Once creep data is obtained under relatively short term tests, the following models are often applied to try to extrapolate the data to obtain predicted lifetimes under longer-term tests. This is obviously very attractive as it saves the necessity to perform extremely long-term creep tests of the order of $10^{5}$ hrs that would represent service conditions.

\section{Empirical Stress and Temperature Dependence Models}

\section{Minimum Creep Rate Calculations}

Perhaps the most common equation used for the calculation of the minimum or steady state creep rate is the Norton creep law [Norton'39]:

$$
\varepsilon^{\prime}=A \sigma^{n}
$$

where $\mathrm{A}$ and $\mathrm{n}$ are constants. The constant $\mathrm{n}$ has already been mentioned with reference to the stress dependence of creep deformation in section 2.2. At low stresses, $n$ is about 1 , for intermediate stresses in pure metals $\mathrm{n}$ is about 4 , and at very high stresses the law breaks down. To account for these discrepancies, a modified version of the above equation has been proposed [Garofa.'65] which has the form:

$$
\varepsilon^{\prime}=C[\sinh (\tau \sigma)]^{\mathrm{n}}
$$

To describe the effect of temperature on creep rate an Arrhenius type expression has been proposed [Dorn'54].

$$
\varepsilon^{\prime}=A_{0} \exp (-Q / R T)
$$

where $A_{0}$ is a constant, $Q$ is equal to the activation energy, $R$ is the universal gas constant and $T$ is the temperature in Kelvin. The value of the activation energy is similar to that of lattice self diffusion at high temperatures, whereas at low temperatures it is similar to that of grain boundary diffusion (approx. half the lattice diffusion value). This reflects the change in 
deformation mechanism between Nabarro-Herring and Coble creep, or the change in vacancy creation by lattice or pipe diffusion under dislocational creep mechanisms at higher stresses.

\section{Empirical Prediction of the Time to Failure}

One of the most common equations used to determine the time to failure is the Monkmann Grant formulation [Monkma.'56]. The observation was made that the time to rupture is proportional to the minimum or steady state creep rate, such that $C_{M C_{i}}=\varepsilon^{\prime} \cdot \mathrm{t}_{\mathrm{f}}$, where $C_{M G}$ is the Monkmann Grant parameter. This relationship is observed in many materials, and is often used as a reference against which creep cavity growth models are compared.

Parametric equations exist which attempt to extrapolate data by the production of graphs plotting stress against a temperature compensated time axis, thus placing all data from different test conditions on one curve. Examples of this type of curve are the Orr-Sherby-Dorn [Orr'54] and Larson Miller [Larson'52] graphs, which are particularly useful for comparing different materials. The latter has the form given below:

$$
P(s)=\left(C+\log t_{f}\right) \cdot T / 1000
$$

where $P(s)$ is the Larson Miller Parameter, $C$ is a constant (often approx. 20), $t_{f}$ is the rupture time and $\mathrm{T}$ is the temperature in Kelvin. The stress dependence is determined empirically.

\section{Empirical Modelling of the Whole Creep Curve (Theta Projection)}

It has been argued that prediction of the creep behaviour based only on the steady state creep rate, the time to failure and even the strain to failure is insufficient to correctly describe the creep curve shape, and hence the creep processes involved. Evans et al. describe the curve shape as the sum of a decaying primary and an accelerating tertiary stage, with the minimum creep rate being the point of inflexion between'these two functions [Evans'82]. The resulting model, termed theta projection, has the following form:

$$
\varepsilon=\theta_{1}\left(1-\exp \left(-\theta_{2} t\right)\right)+\theta_{3}\left(\exp \left(\theta_{4} t\right)-1\right)
$$

$\theta_{1}$ and $\theta_{3}$ scale the extent of primary and tertiary creep respectively, while $\theta_{2}$ and $\theta_{4}$ are shape parameters that describe the curvature of the primary and tertiary stages respectively. Use of the model involves fitting constant stress creep curves with the above equation by using a least squares technique. For each stress level and each temperature a different set of theta parameters is acquired. However, the theta values are a function of stress and temperature as shown below: 


$$
\ln \left(\theta_{i}\right)=a_{i}+b_{i} T+c_{i} \sigma+d_{i} \sigma T
$$

where a,b,c,d are constants and $\mathrm{T}$ is the absolute temperature. The above linear equation then allows extrapolation of the theta parameters, and hence curve shape, to lower stress tests at a given temperature. The time to failure is given as the time to reach some limiting strain value. Several investigators have successfully used this technique for a variety of materials and conditions (see for example [Loghma.'96],[Taylor'90],[Evans'82],[Hayhur.'85]).

\section{Phenomenological Models}

\section{An Introduction to Continuum Damage Mechanics (CDM)}

Phenomenological modelling involves identifying the main important parameters and then formulating some equation into which they can be used effectively. In this way, they are a blend of pure empirical and mechanistic modelling approaches. The following discussion is based around the "damage" parameter first introduced by Kachanov to categorise creep brittle fracture [Kachan.'58]. The parameter varies between a value of 0 for virgin material, and 1 at failure, and is assumed to follow the kinetic law given below:

$$
\frac{\mathrm{d} \omega}{\mathrm{dt}}=\mathrm{A}\left(\frac{\sigma}{1-\omega}\right)^{v}
$$

where $A$ and $V$ are constants. Integration of the above equation between the limits of $\omega=0$ to 1 when $t=0$ to $t_{f}$ gives an expression for the rupture lifetime. However, following work by Rabotnov, equation (2.17) is more usually used in combination with a power law creep relation in what has become known as continuum damage mechanics [Rabotn.' 69], the generalised equations of which are given below:

$$
\frac{\mathrm{d} \varepsilon}{\mathrm{dt}}=\mathrm{B} \frac{\sigma^{\mathrm{n}}}{(1-\omega)^{\mathrm{r}}} \quad \frac{\mathrm{d} \omega}{\mathrm{dt}}=\mathrm{A} \frac{\sigma^{\nu}}{(1-\omega)^{\mathrm{x}}}
$$

where $A, B, n, v, r, x$ are material constants.

Solving the above equations together leads to an expression for the strain accumulation with time during the tertiary creep phase. The applied stress is assumed to be constant. The theory is based on the idea that failure occurs due a progressive internal deterioration of the material, which induces a loss of strength leading to increased strain rates. Solving the equations requires 
determining the six constants. Leckie et al. suggest that the constants may be found from a series of uniaxial creep tests [Leckie'74b], but it is often assumed that $n=r$ and $v=x$, which greatly simplifies the solutions.

\section{Development of CDM Concepts}

The above equations can be found in several different forms, and have been applied to a range of situations including fatigue [Chaboc.'74], creep-fatigue interaction [Chaboc.'81], and multiaxial stress states [Leckie'74]. Tensorial representations of damage have also been developed which are meant to describe more accurately the damage evolution, which is not strictly isotropic. However, the increasing number of parameters required makes the model cumbersome, and so the scalar parameter $\omega$ is normally used. Perhaps the most applicable development of the CDM concept to the current work is that of Dunne et. al. [Dunne'90], who incorporate a time dependent algorithm into their equations to account for primary creep:

$$
\frac{\mathrm{d} \varepsilon}{\mathrm{dt}}=\mathrm{Kt}^{-m}\left(\frac{\sigma}{1-\omega}\right)^{\mathrm{n}} \quad \frac{\mathrm{d} \omega}{\mathrm{dt}}=\mathrm{At}^{-\mathrm{m}} \frac{\sigma^{v}}{(1-\omega)^{\phi}}
$$

The use of such a time dependence of primary creep has been disputed, since it is often assumed that a strain hardening mechanism is dominant in the early stages. Some justification for time hardening can be drawn from the fact that some precipitate hardened materials may not be fully hardened at the beginning of their creep life, and undergo further microstructural changes which cause hardening as the material ages. However, mechanistic arguments aside, the above equations have been successfully applied to fit creep curves in a range of materials [Dunne'90], [Church'92]. Further development of these equations has lead to a model for creep under constant load conditions, by replacing the stress term $\sigma$ with the true stress $\sigma_{\text {true }}=\sigma_{0} \exp (\varepsilon)$, where $\sigma_{0}$ is the initial stress, and $\varepsilon$ is the true strain [Church'92]. However, solving the equations then becomes increasingly difficult, and numerical techniques are required to fit the creep curves.

\section{Application to Remaining Life Assessment}

Solution of equations (2.18) or (2.19) provide a relationship between strain and time such that creep curves may be drawn and fitted to experimental data. Assuming that there is no change in creep mechanism, extrapolation of the curves to lower stresses may be performed. However, industrial plants may operate under varying operating conditions, which can significantly 
change the life expectancy. To overcome this problem, Robinson showed that integration over the various conditions allowed good prediction of behaviour [Robins.'52]. This concept is known as the life fraction rule, which states that creep damage under a given set of conditions is proportional to the fraction of the expected creep life spent under those conditions. Summing up over all the operating conditions gives the total damage in the material.

Application of the CDM technique to remaining life assessment has been carried out by Cane, who showed that rupture life in low alloy ferritic steel tubes and pipes is insensitive to the rupture strain, and depends chiefly on the steady state or minimum creep rate, as implied by the Monkmann Grant relationship [Cane'82]. Other researchers have had success with the CDM concept, particularly for low alloy steels e.g. [Braam'94],[Hayhur.'84],[Taylor'90].

\section{Physical Interpretation of the Damage Parameter}

One of the main attractions of the CDM approach is the possibility to relate the damage parameter to some physical event. Rabotnov specifically avoided any such interpretation [Rabotn.'69], and Leckie et. al have shown that a physical representation of damage is not necessary to form accurate and valid constitutive equations [Leckie'75]. Nevertheless, the number fraction of cavitating grain boundary facets has been related to the damage parameter by Cane et al [Cane'85], and Shammas [Shamma.'87]. A similar, modified parameter valid for low cavity densities was introduced by Hutchinson [Hutchi.'83], with further development extending this work to higher densities [Riedel'89]. The omega parameter is often also considered as a reduction of the effective specimen cross sectional area.

Damage relations other than cavitation have been proposed by Ashby and Dyson, who developed CDM relations for eight different mechanisms [Ashby'84]. It is then necessary to work out which mechanism is controlling since superposition of two or more mechanisms is difficult to model with this technique.

\section{Multiaxial Stress Considerations}

In most industrial situations, components are subjected to complex multiaxial stresses, and thus the models presented so far for use with uniaxial data are inadequate. To asses component behaviour, a method must be found which can reliably relate the uniaxial data to the multiaxial case. The complex stresses are therefore normally broken down to a representative single stress, which can be compared with the standard uniaxial stress. Two characterisations are commonly 
used for the study of creep behaviour: that governing the deformation behaviour, resulting in a multiaxial stress deformation criterion (MSDC), and that governing rupture, with a corresponding multiaxial stress rupture criterion (MSRC).

\section{Fundamentals of The Multiaxial Stress State}

The stress state in a body can be described by nine components of normal and shear stress. A unique set of orthogonal axis exist for which the stress state may be simply defined in terms of the three normal principal stresses, acting along the principal axis. In tensorial form, the stress state can then be represented as:

$$
\left[\begin{array}{ccc}
\sigma_{1} & 0 & 0 \\
0 & \sigma_{2} & 0 \\
0 & 0 & \sigma_{3}
\end{array}\right]
$$

where $\sigma_{1}, \sigma_{2}, \sigma_{3}$ are the principal stresses. These principle stresses are often considered as the sum of two different stresses, namely the hydrostatic $\left(\sigma_{H}\right)$ and deviatoric $\left(S_{\mathrm{i}}\right)$ stress, where:

$$
\sigma_{\mathrm{H}}=\frac{\sigma_{1}+\sigma_{2}+\sigma_{3}}{3} \quad \text { and } \quad S_{i}=\sigma_{i}-\sigma_{H}
$$

It has been found that the hydrostatic stress is responsible for volumetric changes, but not shape changes, whereas the deviatoric stress is responsible for creating shear stresses and deformation.

\section{Representative Multiaxial Stresses}

As mentioned earlier, an expression for a single stress to represent the multiaxial state is necessary for analysis of the material behaviour. Whilst several proposals have been made, the two most commonly used criteria are the Von Mises effective stress [VonMis.'13] and the Tresca maximum shear stress [Tresca ${ }^{18} 64$ ]. Originally developed for plasticity theory rather than creep analysis, they give a relationship between the critical stress to cause yielding under uniaxial conditions and a corresponding representative stress under multiaxial conditions. The proportionality at yield is considered to extend beyond it.

The Von Mises effective stress states that yielding occurs when the shear strain energy of a system of principal stresses reaches a critical value. The effective stress is then defined as: 


$$
\sigma_{\mathrm{e}}=\frac{1}{\sqrt{2}}\left[\left(\sigma_{1}-\sigma_{2}\right)^{2}+\left(\sigma_{2}-\sigma_{3}\right)^{2}+\left(\sigma_{3}-\sigma_{1}\right)^{2}\right]^{1 / 2}
$$

with a corresponding equivalent strain given by:

$$
\varepsilon_{\mathrm{e}}=\frac{\sqrt{2}}{3}\left[\left(\varepsilon_{1}-\varepsilon_{2}\right)^{2}+\left(\varepsilon_{2}-\varepsilon_{3}\right)^{2}+\left(\varepsilon_{3}-\varepsilon_{1}\right)^{2}\right]^{1 / 2}
$$

Alternatively, the Tresca effective stress correlates the maximum shear stress in the body with yielding, such that:

$$
\sigma_{\mathrm{e}}=\tau_{\max }=\frac{\sigma_{1}-\sigma_{3}}{2} \quad \text { and } \quad \varepsilon_{\mathrm{e}}=\gamma_{\max }=\varepsilon_{1}-\varepsilon_{3}
$$

These representative stresses may now be used to formulate the MSDC and MSRC such that the modelling techniques discussed earlier may be applied to the multiaxial case.

\section{Multiaxial Stress Deformation Criterion}

The extension of the effective stresses to creep deformation was initially developed by Soderberg [Soderb.'36]. Assuming the material to be isotropic and homogenous, several assumptions were made:

1. The directions of the principal extensions coincide with those of the principal stresses

2. The density or volume of the material remains constant

3. The three principal shear strains are proportional to the three principal shear stresses

4. The yielding follows the Von-Mises criterion in which the strain energy is the determining variable

For creep deformation the effective strain rate is given as:

$$
\dot{\varepsilon}_{\mathrm{e}}=\frac{\sqrt{2}}{3}\left[\left(\dot{\varepsilon}_{1}-\dot{\varepsilon}_{2}\right)^{2}+\left(\dot{\varepsilon}_{2}-\dot{\varepsilon}_{3}\right)^{2}+\left(\dot{\varepsilon}_{3}-\dot{\varepsilon}_{1}\right)^{2}\right]^{1 / 2}
$$

Making the further assumption that the effective strain rate can be related to the effective stress in a similar way to that used for the uniaxial case, then the following expression can be derived for the principal strain rates: 


$$
\dot{\varepsilon}_{\mathrm{i}}=\frac{3}{2} \frac{\dot{\varepsilon}_{\mathrm{e}}}{\sigma_{\mathrm{e}}}\left(\sigma_{1}-\sigma_{\mathrm{H}}\right)
$$

Substituting for example a Norton power law for the effective strain rate in equation (2.26) gives a general multiaxial creep constitutive rate equation:

$$
\dot{\varepsilon}_{\mathrm{i}}=\frac{3}{2} \mathrm{~A} \sigma_{\mathrm{e}}^{\mathrm{n}-1} \mathrm{~S}_{\mathrm{i}}
$$

The Von Mises criterion is generally considered the most meaningful deformation descriptor in terms of theory and experiment [Johnso.'51], and has been well applied to the case of low alloy ferritic steels [Cane'82], [Church'92].

\section{Multiaxial Creep Ductility}

Creep ductility under multiaxial stressing tends to be reduced compared with the uniaxial case. It has been proposed that under pure hydrostatic tension, a material will behave in an extremely brittle manner, whereas maximum ductility is achieved for the reverse situation under pure hydrostatic pressure [Davis'59]. Taking this idea further, a triaxiality factor was proposed which is proportional to the hydrostatic stress divided by the effective stress. An exact relation between the triaxiality factor and the theoretical ductility ratio (multiaxial ductility/uniaxial ductility) was not given. However, the ductility ratio is expected to be the inverse of the triaxiality factor. The sensitivity of the ductility ratio for a given steel is related to the ' $n$ ' value, with higher values giving more sensitive response [Manjoi.'75]. For internally pressurised tubes, a ductility ratio of about $2 / 3$ is often expected.

\section{Multiaxial Stress Rupture Criterion}

In order to predict the failure time of components under multiaxial stresses, a representative stress, termed the multiaxial stress rupture criterion is necessary. The MSRC tends to involve a combination of effective (shear stresses), and the maximum principal stress (equivalent to the Tresca criterion under plain stress conditions). This reflects the dependence of the creep life on both deformation and damage processes. The particular criterion dominant in a given material can sometimes be ascertained from the failure sections, with plastic failures being associated with the effective stress, and more brittle failures generally associated with the maximum principal stress [Johnso.'60]. 
In the case of failure by cavity coalescence, it has been suggested that cavity nucleation is controlled by shear stresses, for which the effective stress is controlling, whereas cavity growth occurring predominantly by diffusion is controlled by the maximum principal stress (MPS) [Dyson'76b]. Further work showed that the MSRC depends upon many factors including temperature, microstructure and grain size, as well as on the particular cavity growth mechanism dominant under the given conditions [Cane'81b]. In ferritic steels a transition in controlling stress from effective to MPS with decreasing stress has been observed, which may reflect the tendency for materials to fail in a more brittle manner with increasing time [Nix'89],[Needha.'80]. However, the exact reasons for why a particular MSRC works in some situations and not others is still rather unclear.

To account for these difficulties, the MSRC is often described using empirically derived equations that can be fitted to experimental data. One such MSRC uses a combination of the MPS and the Von Mises stress [Hayhur.' 84 ]:

$$
\sigma_{\mathrm{MSRC}}=\alpha \sigma_{1}+(1-\alpha) \sigma_{\mathrm{e}}
$$

where $\alpha$ is a constant between zero and one. More complex rupture criteria have been developed based upon the hydrostatic and octahedral stresses, as well as the stress invariants of the principal stresses [Hayhur.'78].

\section{Multiaxial Stress Analysis For Tubular Components}

To apply the MSDC and the MSRC to real components it is necessary to know the values of the principal stresses at a given position within the component. Modern computational and finite element techniques may be used for complex shapes, but for the case of a tube under internal pressure, the following analytical solutions exist for the elastic stress distribution [Lame ${ }^{18} 52$ ]:

$$
\sigma_{\theta}=\frac{\operatorname{Pr}_{i}^{2}}{r_{o}^{2}-r_{i}^{2}}\left[1+\frac{r_{0}^{2}}{r^{2}}\right]: \quad \sigma_{r}=\frac{\operatorname{Pr}_{i}^{2}}{r_{o}^{2}-r_{i}^{2}}\left[1-\frac{r_{0}^{2}}{r^{2}}\right]: \sigma_{z}=\frac{\operatorname{Pr}_{i}^{2}}{r_{o}^{2}-r_{i}^{2}}
$$

where $r_{i}$ and $r_{0}$ are the inner and outer tube radii respectively, $r$ is a radial position within the tube, $\mathrm{P}$ is the internal pressure, and $\sigma_{0}, \sigma_{\mathrm{r}}, \sigma_{\mathrm{z}}$ are the hoop, radial and axial stresses respectively. However, under creep conditions, stress redistribution takes place. Assuming that the Norton law is representative of creep behaviour, then the equations can be re-written [Bailey'51] as shown in equations 2.30 , where $\mathrm{n}$ is the Norton stress exponent. 


$$
\sigma_{\mathrm{r}}=\mathrm{P} \frac{1-\left(\frac{\mathrm{r}_{0}}{\mathrm{r}}\right)^{2 / n}}{\left(\frac{\mathrm{r}_{0}}{\mathrm{r}_{\mathrm{i}}}\right)^{2 / n}-1} \quad \sigma_{\mathrm{r}}=\mathrm{P} \frac{\frac{1-\mathrm{n}}{\mathrm{n}}\left(\frac{\mathrm{r}_{0}}{\mathrm{r}}\right)^{2 / n}+1}{\left(\frac{\mathrm{r}_{0}}{\mathrm{r}_{\mathrm{i}}}\right)^{2 / n}-1} \quad \sigma_{0}=\mathrm{P} \frac{\frac{2-\mathrm{n}}{\mathrm{n}}\left(\frac{\mathrm{r}_{0}}{\mathrm{r}}\right)^{2 / n}+1}{\left(\frac{\mathrm{r}_{0}}{\mathrm{r}_{\mathrm{i}}}\right)^{2 / n}-1}
$$

Figure 2.11 compares the creep and elastic stress distributions across the wall thickness of a tube. Under creep conditions the maximum principle stress is seen to move to the outer surface of the tube, whilst the Von Mises stress is always a maximum at the inner wall.

The creep stress distribution shown above is calculated to be accurate under steady state conditions. The time to reach such a steady state has been shown by several authors to be relatively rapid compared with the time to failure (e.g. [Johnso.'61]), with some evidence to suggest it approximates to the extent of primary creep [Taira'68]. In such caśes steady state conditions can often be safely assumed to last throughout most of the creep life. However, at lower stresses the redistribution time may persist longer in some materials, causing difficulties in analysis.

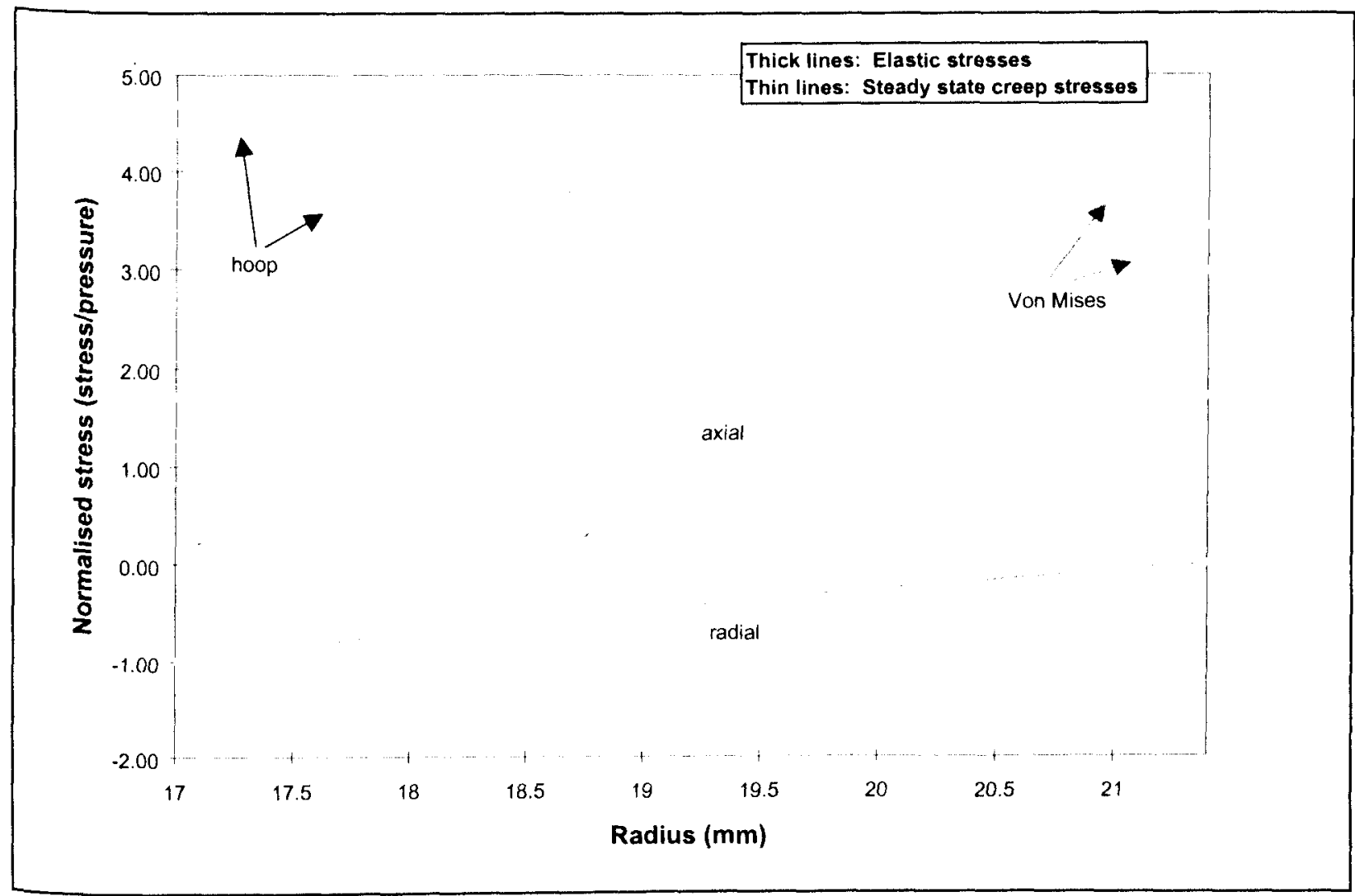

Figure 2.11: Comparison of the elastic and steady state creep stress distributions predicted by equations (2.29) and (2.30) for an internally pressurised tube with inner radius $17 \mathrm{~mm}$, and outer radius $21.4 \mathrm{~mm}$ 
The object of the proceeding discussion on multiaxial creep behaviour centred around the desire to find a single representative stress which would cause a uniaxial specimen tested at this stress to respond in a similar way to the multiaxially stress component. Considering then the stress distribution that exists within a component, it is necessary to find a point at which this reference stress can be taken. Examination of figure 2.11 reveals a position at about half the wall thickness where the elastic and creep stress profiles pass through the same point. This point is independent of $n$, does not change with stress redistribution, and is termed the skeletal point. The stress measured at the skeletal point is often conveniently used as the reference stress for tubular components. However, the skeletal point stress and reference stress may not be equal for more complex geometries. Calculation of the skeletal point stress is given below for the Von Mises effective stress distribution, although under high stress conditions it may be better to use the mean diameter hoop stress [Cane'82b]:

$$
\sigma_{\mathrm{SP}}=\frac{P \sqrt{3}}{K^{2}-1}\left(\frac{K^{2}-1}{n\left(K^{2 / n}-1\right)}\right)^{n / n-1} \quad \text { where } K=\frac{r_{o}}{r_{i}}
$$

An alternative formulation for the reference stress in thick wall tubes has been developed as shown below [Soderb.'41]:

$$
\sigma_{\text {ref }}=\mathrm{P} \frac{\sqrt{3}}{2} \frac{r_{\mathrm{m}}}{\mathrm{t}}
$$

\section{CDM Modelling Under Multiaxial Loading}

When the appropriate MSDC and MSRC have been found at the correct reference point, the CDM equations detailed earlier can be generalised to account for the multiaxial stress state. Combining equation (2.27) with (2.19) gives the following dependence of strain rate on stress state:

$$
\varepsilon_{i}=\frac{3}{2} K t^{-m} \frac{\sigma_{\mathrm{e}}^{n-1}}{(1-\omega)^{n}} S_{i}
$$

Similarly, the damage evolution law has been modified to the following form [Dunne'90]:

$$
\omega=\frac{\mathrm{Bt}^{-m} \sigma_{\mathrm{MSRC}}^{\nu}}{(\phi+1)(1-\omega)^{\phi}}
$$


which reduces to the uniaxial form given that $\mathrm{A}=\mathrm{B} /(\phi+1)$. Assuming that the correct multiaxial criterion are chosen and that the six material parameters have been found for a uniaxial data set, then prediction of multiaxial creep should be possible from application of the above equations.

\section{Creep Modelling in Aggressive Environments}

The phenomenological models presented previously are normally used for predicting creep in relatively benign situations. They are however adaptable to more aggressive situations, such as oxidising and carburising environments. Ashby and Dyson give various forms of the CDM equations for internal and surface oxidisation [Ashby'84]. For example, with internal oxidation a front of damage is assumed to grow into the material with certain kinetics. The oxidised layer is assumed to carry no load, so it acts as a loss of external section.

A similar approach was used by Taylor in a carburisation study of alloy $800 \mathrm{H}$ [Taylor'87]. In this case the carburised layer was not treated as having zero strength, but rather as having a different strength from the base material. The resulting model resembled a two bar loading situation in which the stress is distributed between the two different material layers. Taylor developed the model to account for internal carburisation of pressurised tubes, where the stress state was multiaxial. The tube was split into concentric cylinders, the inner one which had properties of carburised material, and the outer which behaved as the base material. A similar approach is used in section 6 of this thesis. Additional kinetic modelling allowed the depth of the carburised front to be changed with time.

The creep models for oxidation and carburisation mentioned above are valid for surface changes on the creep specimens. This is similar to the effect of surface decarburisation in hydrogen attack. HA cavitation on the other hand is a homogenous degradation throughout the specimen thickness (for hydrostatic exposure conditions), and must therefore be considered differently. Mechanistic models treat hydrogen attack as a creep cavity growth problem, accelerated by an internal methane pressure. Ashby and Dyson. [Ashby'84] and Cocks and Ashby [Cocks'82] have presented CDM models specifically for creep cavitation. Two forms exist: one covering diffusional cavity growth, and one for power law creep cavity growth. As far as the author is aware, no-one has tried modifying these with an internal methane pressure. 


\subsection{Conclusions From The Literature Review}

It is clear that the general mechanisms of hydrogen attack are understood. For simple cases of unstressed material, it is even possible to model the behaviour, either mechanistically or empirically, such that an effective lifetime can be predicted. It is also apparent that the application of a stress to a steel undergoing hydrogen attack can accelerate the attack mechanisms. Furthermore, interaction can occur between creep cavitation mechanisms and hydrogen attack cavitation mechanisms, demonstrated both theoretically and experimentally. Models of hydrogen attack behaviour under an applied stress have been developed. However, lack of experimental data means that these models are yet to be accurately verified and it is not clear just how much of an effect stress can have on hydrogen attack cavitation. As a result, prediction of industrial components is still generally based around the Nelson curves, which are not an ideal design tool.

Most hydrogen attack models are concerned with predicting the cavity growth rate, and defining some point at which the cavities coalesce, thereby causing failure. Similar models exist, and have been successfully applied to the case of creep cavitation. Despite this, much remaining life assessment and creep behaviour prediction relies on empirical and macroscopic phenomenological modelling approaches, which can model the overall creep strain accumulation with time for a given component. These creep models can also be well adapted to multiaxial stress states and, as such, are useful for component behaviour prediction. However, as yet, there has been little or no attempt to apply these models to the case of combined creep and hydrogen attack. It is believed that this is not because the modelling approach will not work, but rather that the expense and difficulty of performing the necessary experiments under high-pressure and temperature hydrogen has limited the amount of data available, particularly for the multiaxial case.

Empirical and phenomenological modelling overcomes certain problems relating to accurate determination of a large number of material constants. However, it is generally accepted that even the most empirical models are only valid for certain mechanistic regimes of the component lifetime. The need then arises to understand better the detailed mechanisms of hydrogen attack and creep in a given material. Whilst much work has been carried out in $2.25 \mathrm{Cr}-1$ Mo steel, there is a large amount of scatter and uncertainty in the available hydrogen attack data. For example, measured values for the nucleation rates, growth rates and creep strength under 
hydrogen attack conditions do not always correspond very well from one test to another. The problem becomes worse for a steel like $2.25 \mathrm{Cr}-1 \mathrm{Mo}$, for which a large number of heat treatments and minor alloy changes are available. Furthermore, the damage observed during hydrogen attack - creep interaction has been restricted to uniaxial data under hydrostatic hydrogen pressures. Experiments are lacking which observe the damage occurring under more industrially realistic conditions. Greater understanding of the effect of these factors is required to apply the models to a full range of materials. 


\section{AIMS OF THE THESIS}

The following points state the main aims of the thesis. These have been based partially on the conclusions from the literature review (section 2.7). Following the aims of the thesis are a set of methods which will be used in order to obtain the aims.

\section{Main Aims of the Thesis}

1) To produce new test data to help clarify the synergism between combined creep and hydrogen attack interaction. In particular, to use testing methods other than simple uniaxial creep which represent closer the industrial components used in hydroprocessing reactors.

2) To provide more detailed analysis of the damage mechanisms occurring during hydrogen attack and hydrogen attack-creep interaction. The information could be used as input into mechanistic models for hydrogen attack.

3) To develop a method for predicting the lifetime of hydrogen exposed laboratory creep specimens, which can potentially be scaled to the lifetime prediction of industrial components.

\section{Methods}

a) Design and build testing facilities for creep testing in hydrogen environments. A uniaxial creep testing rig is required which can operate in hot hydrogen environments, and hydrogen pressurised tube testing provides conditions which represent closer the industrial situation.

b) Detailed metallographic analysis of test specimens is required to determine the damage mechanisms occurring.

c) Creep curve fitting routines such as theta projection and CDM can be developed to include the effect of hydrogen. Analysis of the test specimens will provide data on how to best incorporate the lifetime predictions.

d) Within the scope of the thesis, it is only possible to study the details of combined creephydrogen attack interaction in one steel. Due mainly to its common usage, the $2.25 \mathrm{Cr}$ - I Mo steel will be studied (see Chapter 1: Introduction). 


\section{EXPERIMENTAL TECHNIOUES}

One of the main stumbling blocks for the study of hydrogen attack has been the difficulty of performing tests under the high temperatures and hydrogen pressures required. A major part of the work in this thesis went towards addressing this problem by the design and validification of two testing rigs for use in hydrogen environments. Both a uniaxial creep test rig and an internally pressurised tube testing rig were built, and are described in this chapter. Also presented here is description of the material for study along with the test specimen design. The method for performing conventional creep tests in air is also briefly described, as this type of test was required for producing baseline data. Finally, the test conditions are for all of the tests are given on a single page for easy reference. 


\subsection{Material and Specimen Preparation}

\section{As Received Material}

In order to provide a base material from which both uniaxial creep specimens and internally pressurised tubular specimens could be machined, it was decided that a long pipe would be the most suitable material form. Approximately six meters of hot finished seamless $2.25 \mathrm{Cr}-1 \mathrm{Mo}$ steel tube to ASTM specification A335-94, grade P22, were ordered from British Steel via a local supplier. The outer diameter of the tube was $48.3 \mathrm{~mm}$, with a wall thickness of $10.16 \mathrm{~mm}$. The material was received in the normalised and tempered form, although no details of the specific heat treatment were given. Normalised and tempered as opposed to quenched and tempered material was preferred as it allowed an easier comparison of creep properties with a similar steel already studied at the JRC [ENEL'97]. In addition, the increased hydrogen attack in normalised and tempered steel as noted in the literature made this the ideal heat treatment to study in short term tests [Shewmo.'82]. The chemical composition and mechanical properties of the chosen steel (according to the manufacturer) are given in tables 4.1 and 4.2 respectively. Also shown in the tables are the ASTM-A335 standard specifications for seamless ferritic alloy-steel pipe for high temperature service [Britis.'86]. Optical microscopy revealed a uniform structure across the thickness of the tube, consisting predominantly of bainite with scattered ferrite grains as shown in figure 4.1. Using the Mean Linear Intercept method, the grain size of this material is estimated at about $16 \mu \mathrm{m}$. From this point on, the aforementioned steel will be referred to as the BS steel.

\begin{tabular}{|r|c|c|c|c|c|c|c|c|c|c|c|c|}
\hline & $\mathbf{C}$ & $\mathbf{S i}$ & $\mathbf{M n}$ & $\mathbf{P}$ & $\mathbf{S}$ & $\mathbf{C r}$ & $\mathbf{M o}$ & $\mathbf{N i}$ & $\mathbf{A l}$ & $\mathbf{C u}$ & $\mathbf{S n}$ & $\mathbf{V}$ \\
\hline $\mathbf{B S}$ & .10 & .21 & .51 & .006 & .01 & 2.18 & .92 & .20 & .005 & .15 & .008 & .007 \\
\hline \multirow{2}{*}{$\mathbf{A 3 5}$} & .15 & .50 & $.30-$ & .030 & .030 & $1.90-$ & .87 & - & - & - & - & - \\
\hline & $\max$ & $\max$ & .60 & $\max$ & $\max$ & 2.60 & 1.13 & - & & & & - \\
\hline
\end{tabular}

Table 4.1: Composition of the British Steel (BS) as supplied pipe and comparison with ASTM-A335 specification

\begin{tabular}{|c|c|c|c|}
\hline & $\begin{array}{c}\text { Tensile Strength } \\
(\mathbf{M P a})\end{array}$ & $\begin{array}{c}\text { Yield Strength } \\
(\mathbf{M P a})\end{array}$ & $\begin{array}{c}\text { Elongation (\%) } \\
(\mathbf{5 0 m m}, \mathbf{m i n})\end{array}$ \\
\hline BS & 582 & 464 & 35 \\
\hline ASTM-A335 & $415 \mathrm{~min}$ & $208 \mathrm{~min}$ & $30 \mathrm{~min}$ \\
\hline
\end{tabular}

Table 4.2: Tensile properties of the British Steel (BS) as supplied pipe and comparison with ASTM-A335 specification 


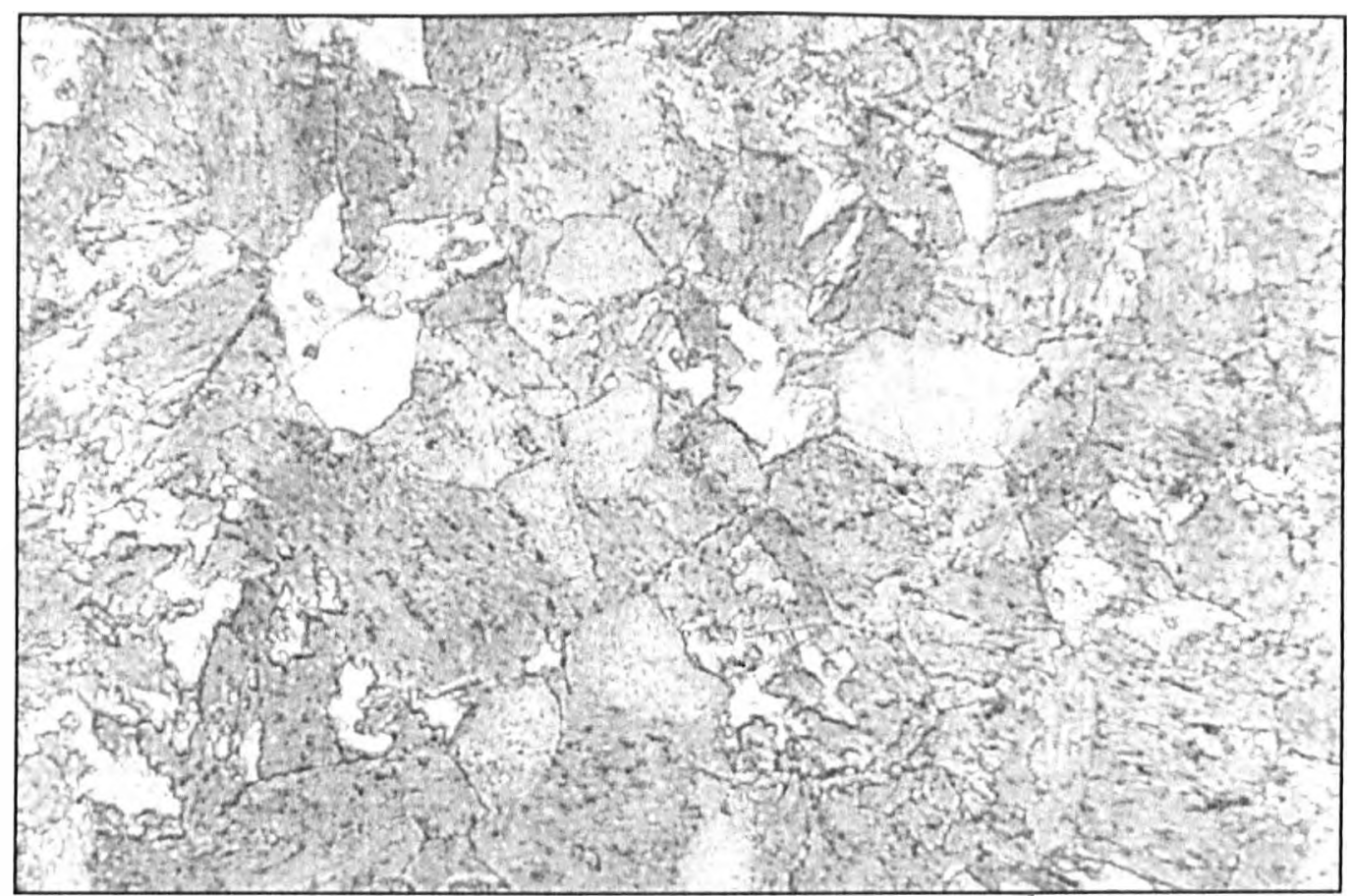

Figure 4.1: Optical micrograph of the as received BS material showing a predominamty bainitic structure with isolated grains of ferrite. (Magnification x400). Specimen etched in $.5 \%$ Nital.

\section{Uniaxial Creep Specimen Design and Preparation}

The creep specimens were designed in accordance with British standard 3500 "Creep and Rupture Testing of Metals" [Britis.'69], which states several dimensional ratios designed to reduce the effect of stress concentrations. The $10 \mathrm{~mm}$ wall thickness of the as-received BS pipe restricted the maximum size of the specimens which could be machined from it, however, spark erosion machining of the specimen blanks allowed very little material to be wasted so that a M10 metric thread could still be machined onto the ends of the specimen (Figure 4.2).

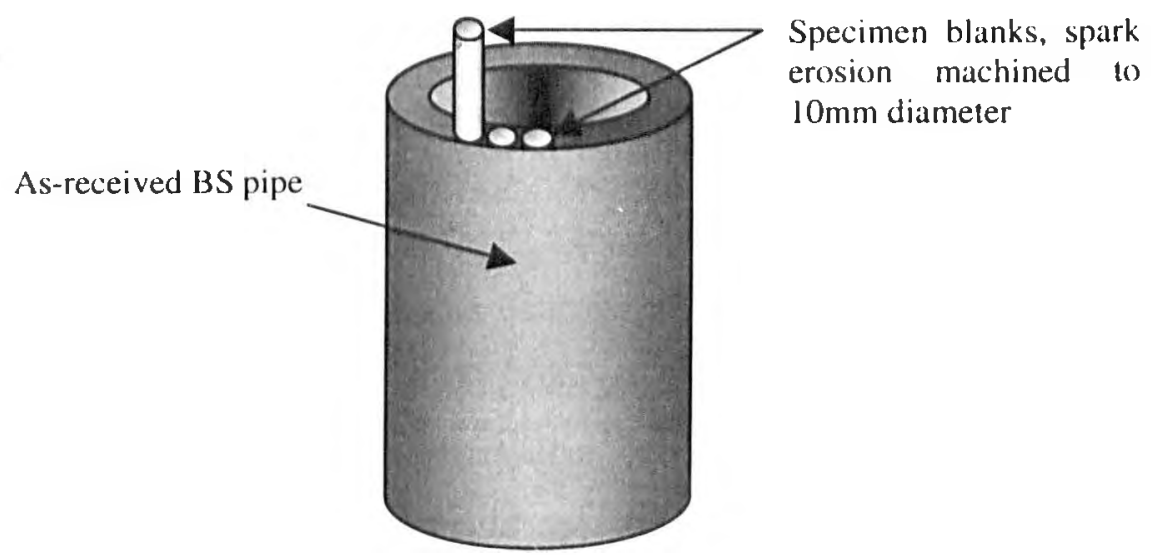

Figure 4.2: Machining of uniaxial specimen blanks from BS pipe 


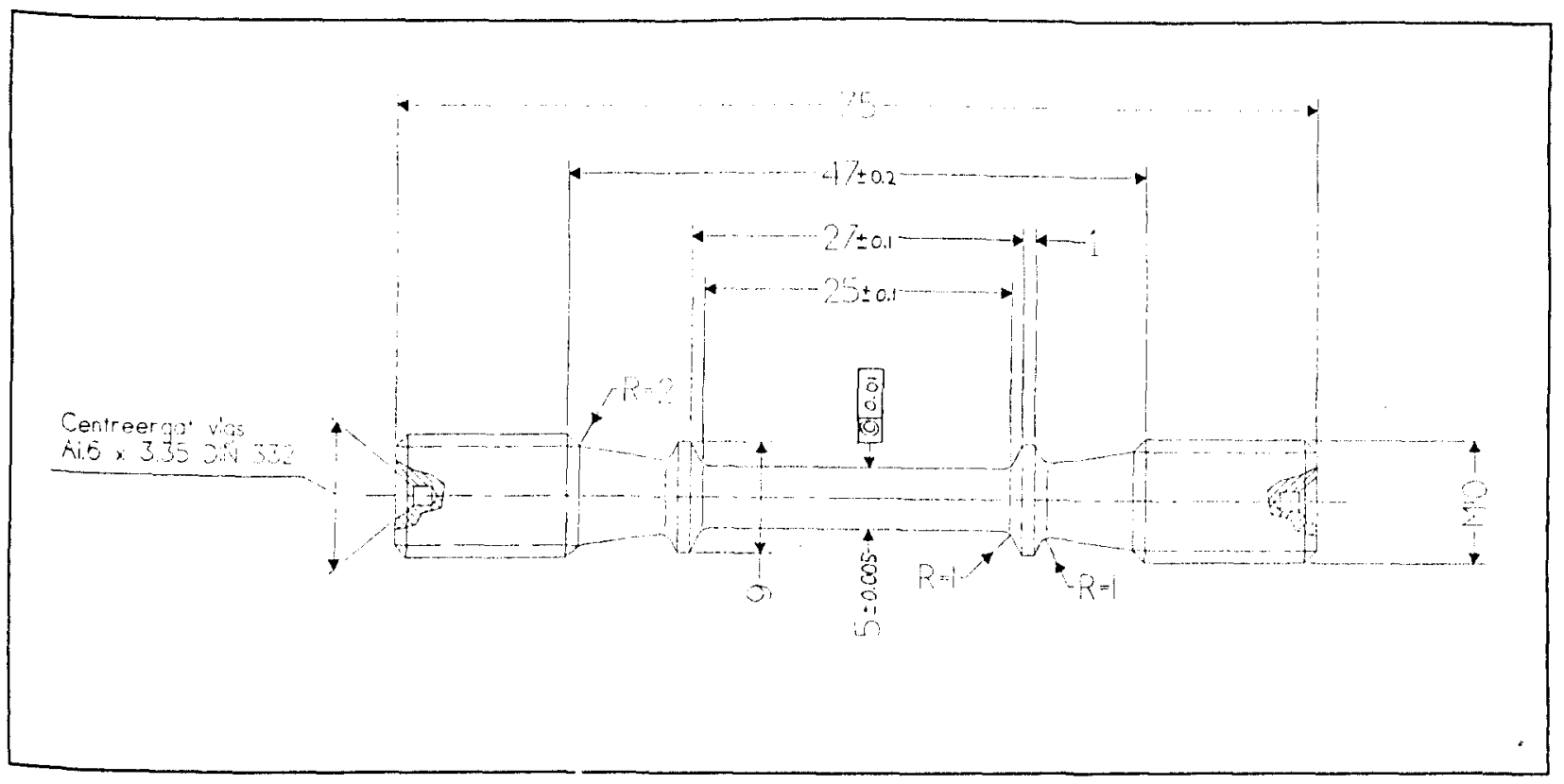

Figure 4.3: Uniaxial creep specimen design for the BS material -

Given the size restrictions and the BS3500 requirements, the final specimen design is given in figure 4.3. The design also includes some knife edges to allow extensometry to be attached to the specimen.

After machining, the specimens were sprayed with vaseline to prevent them from corroding. Specimens were cleaned, given a polish with 800 grit emery paper and finally washed again with acetone prior to testing.

\section{Tubular Specimen Design and Preparation}

The aim was to design specimens that could be tested with high internal pressure (up to approx. $250 \mathrm{bar})$, high temperature $\left(600^{\circ} \mathrm{C}\right)$ hydrogen. The tubes had to conform to the code of practice suggested by the High Temperature Mechanical Testing Committee [HTMTC'89]. In addition, the wall thickness of the tubes was to be as large as possible to ensure a shallow hydrogen concentration gradient across it, such that the effect of the hydrogen could be clearly seen.

\section{Tube Design}

After examination of the literature, it was estimated that the minimum time required to see hydrogen attack in $2.25 \mathrm{Cr}-1 \mathrm{Mo}$ steel at $600^{\circ} \mathrm{C}$ would be around 500 hours in the absence of stress. This time was then taken as a conservative minimum expected test time, assuming that stress would accelerate the attack, and would require the maximum test stress. The maximum 
stress required is important as it limits the specimen thickness for a given maximum internal pressure.

Mean diameter hoop stress-rupture curves of $2.25 \mathrm{Cr}-1 \mathrm{Mo}$ tubular components were examined to determine the stress required to cause failure in about 500 hours (in the absence of hydrogen) [COST'94]. From this data, it was estimated that a mean diameter hoop stress of approximately $110 \mathrm{MPa}$ would be required to cause failure in the given time.

Assuming the conservative figure of 250 bar for the maximum possible test pressure, and knowing the desired mean diameter hoop stress, it was possible to calculate the mean diameter to thickness ratio from the following equation:

$$
\sigma=\frac{P d_{m}}{2 t}
$$

where $P$ is pressure, $d$ is the mean diameter, and $t$ is the thickness. Figure 4.4 shows the code of practice for tubular component design recommended dimensional ratios which are designed to

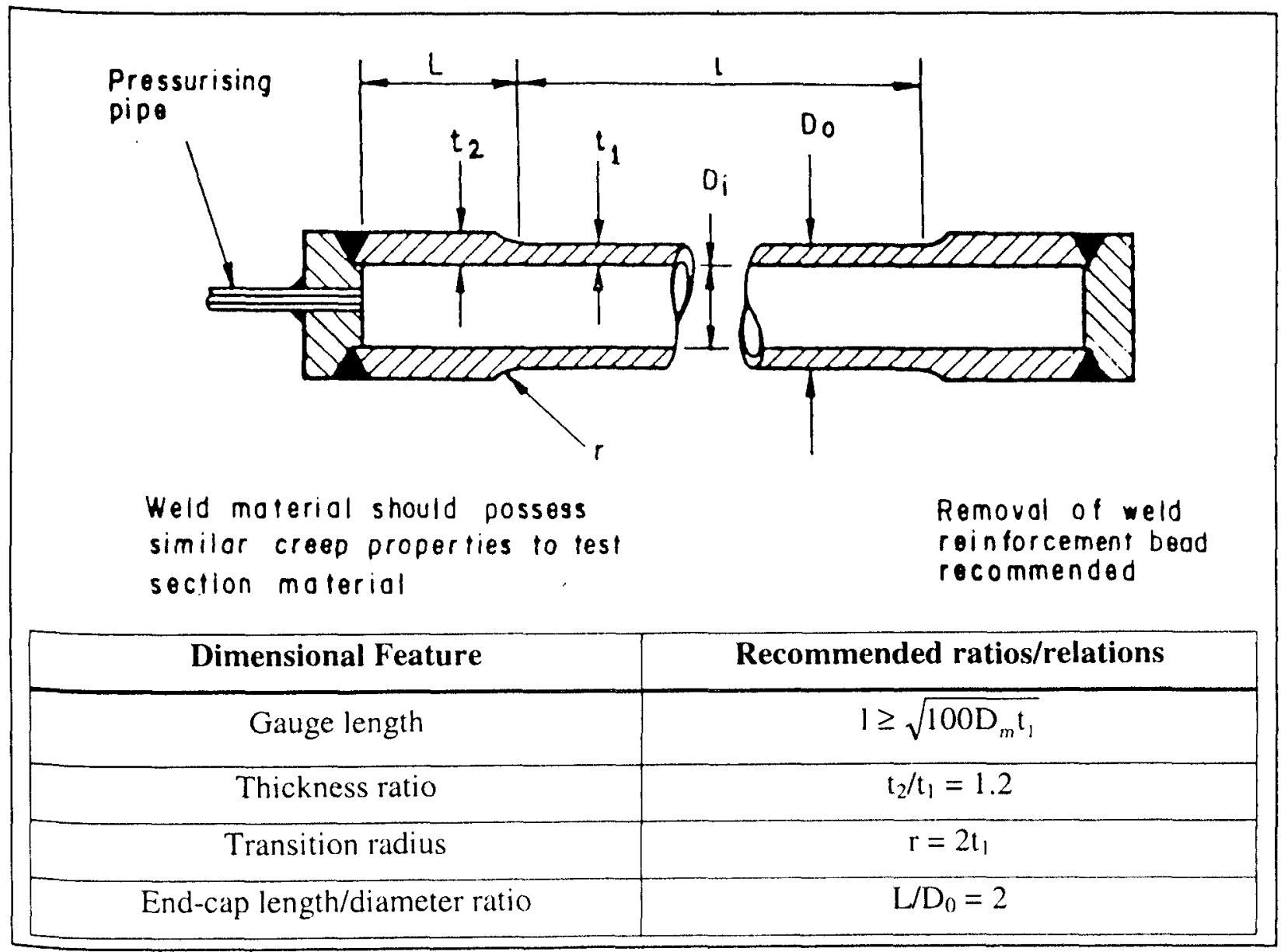

Figure 4.4: Recommended tubular specimen design from the "Code of Practice for Internal Pressure Testing of Tubular Components at Elevated Temperatures" [HTMC'89] 
minimise the effect of stress concentrations. Together with the mean diameter to thickness ratio calculated above, it was possible to draw up a table of a range of different possible dimensions for the tubes. It is however normal practice to use a gauge length somewhat longer than the minimum value suggested. The stress raising effect of the shoulders is minimised when the shoulder thickness is 1.2 times the gauge thickness. However, it has been found by experience at the JRC that a ratio of 1.5 is better as it reduces strain in the shoulder area. It should also be noted that the shoulder length can also be shorter than the recommended $2 \mathrm{~d}_{0}$ without adversely affecting the test.

Several other factors had to be accounted for in the design of the specimens. For example, the outer diameter of the as received BS material was only $48 \mathrm{~mm}$, and the maximum length of the hot zone in the testing rig furnace v'as only $300 \mathrm{~mm}$. Furthermore, under the lowest stress test it was important that the hydrogen pressure was still high enough to cause hydrogen attack. Estimating the maximum possible test time as 10000 hours, the stress required was taken as $75 \mathrm{MPa}$ [COST'94]. Using the same diameter to thickness ratio as before, the estimated

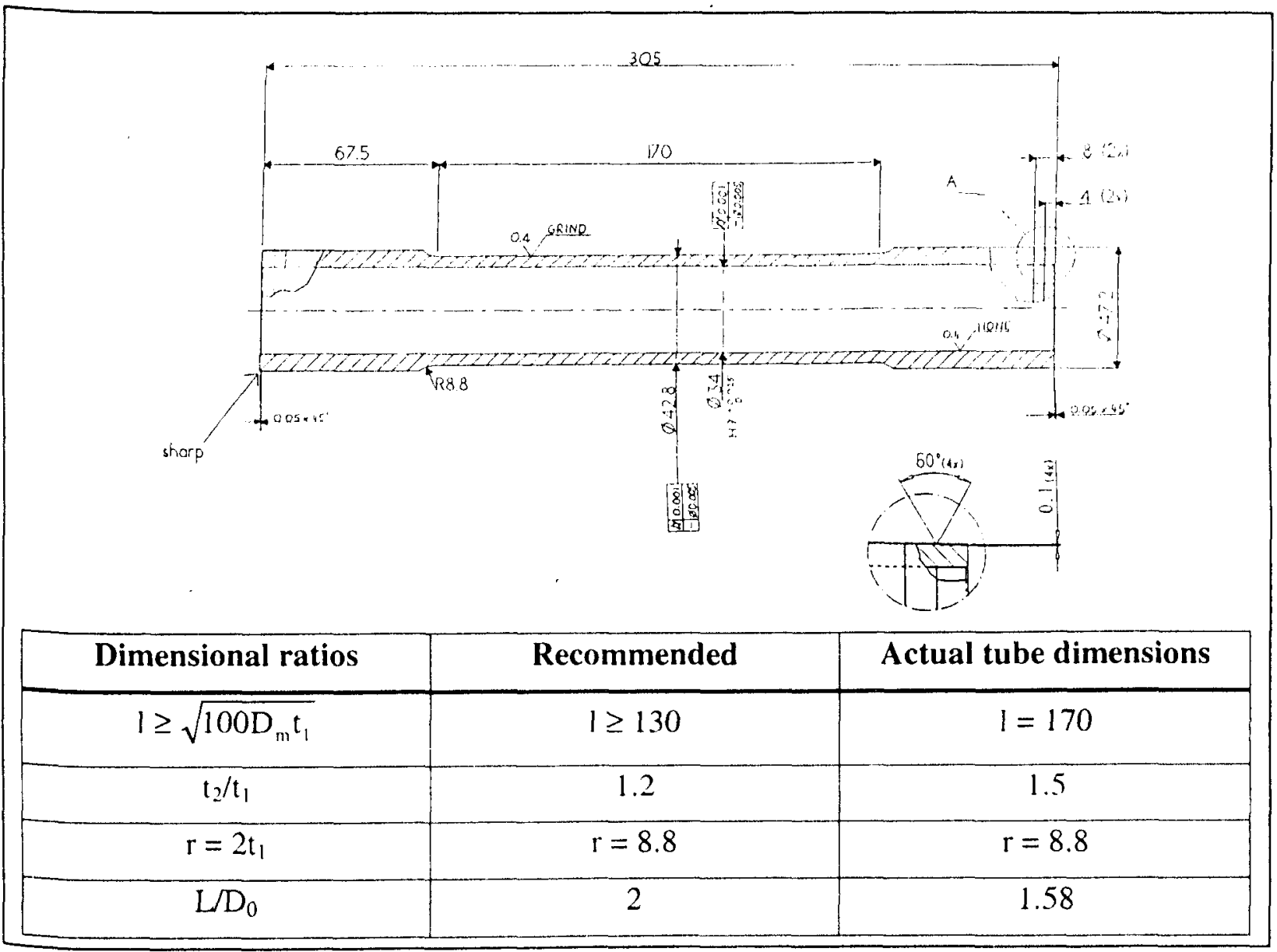

Figure 4.5: Tubular specimen design and comparison of the dimensions with the recommended practice from the High Temperature Mechanical Testing Committee [HTMC'89] 
hydrogen pressure required was 13.6MPa. This was seen to be well above the Nelson curve line for $2.25 \mathrm{Cr}-1$ Mo steel at $600^{\circ} \mathrm{C}$, and so there was no foreseen problem with lack of attack at low stresses [API'97].

The chosen specimen design is shown in figure 4.5, along with a comparison of its' dimensional ratios compared with the recommended practice. The shoulder length was decreased to allow easier machining of the inner tube bore.

\section{End-caps and Gas Supply Pipes}

In order to provide gas tight ends on the specimens, end-caps were designed and machined from a bar of $2.25 \mathrm{Cr}-1 \mathrm{Mo}$ steel, illustrated in figure 4.6. The end-caps were attached to the tubes using electron beam welds which allow a good, high quality weld with a minimum of specimen distortion. In addition to the end caps, gas supply pipes made from $14 \times 8 \mathrm{~mm}$ stainless steel tube were electron beam welded onto the end caps at either end. These pipes were fitted with high pressure fittings to allow safe connection to the gas supply infrastructure.

During testing, a couple of the tubes began to leak hydrogen before failure from the welds on the end-caps. Metallographic examination showed that this was due to lack of penetration of the weld which created a stress concentration at the weld root (figure 4.7). In addition, the weld metal appears to have undergone some hydrogen attack. It was also considered that having three welds next to each other created tensile residual stresses which may have aided in the premature weld failure. As a result, many of the later tubes were welded with only one weld.

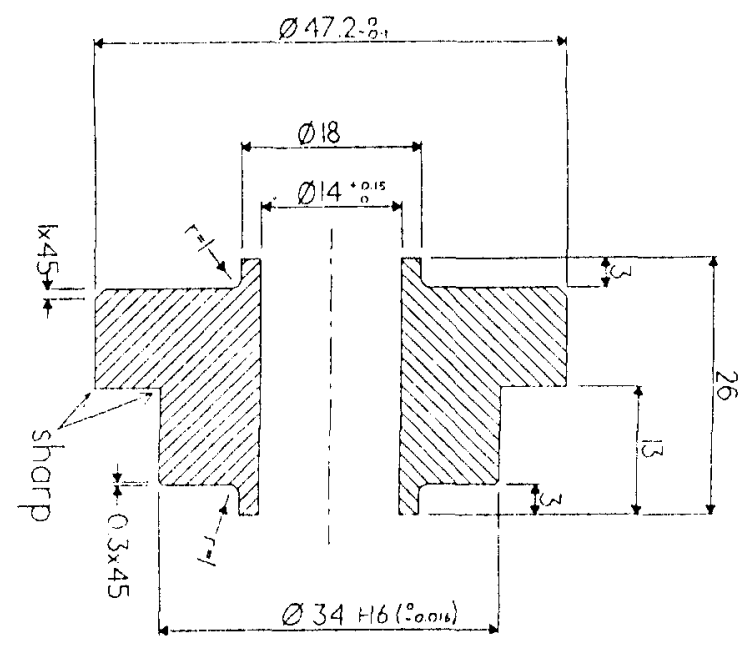

Figure 4.6: End cap design for internally pressurised tubes 


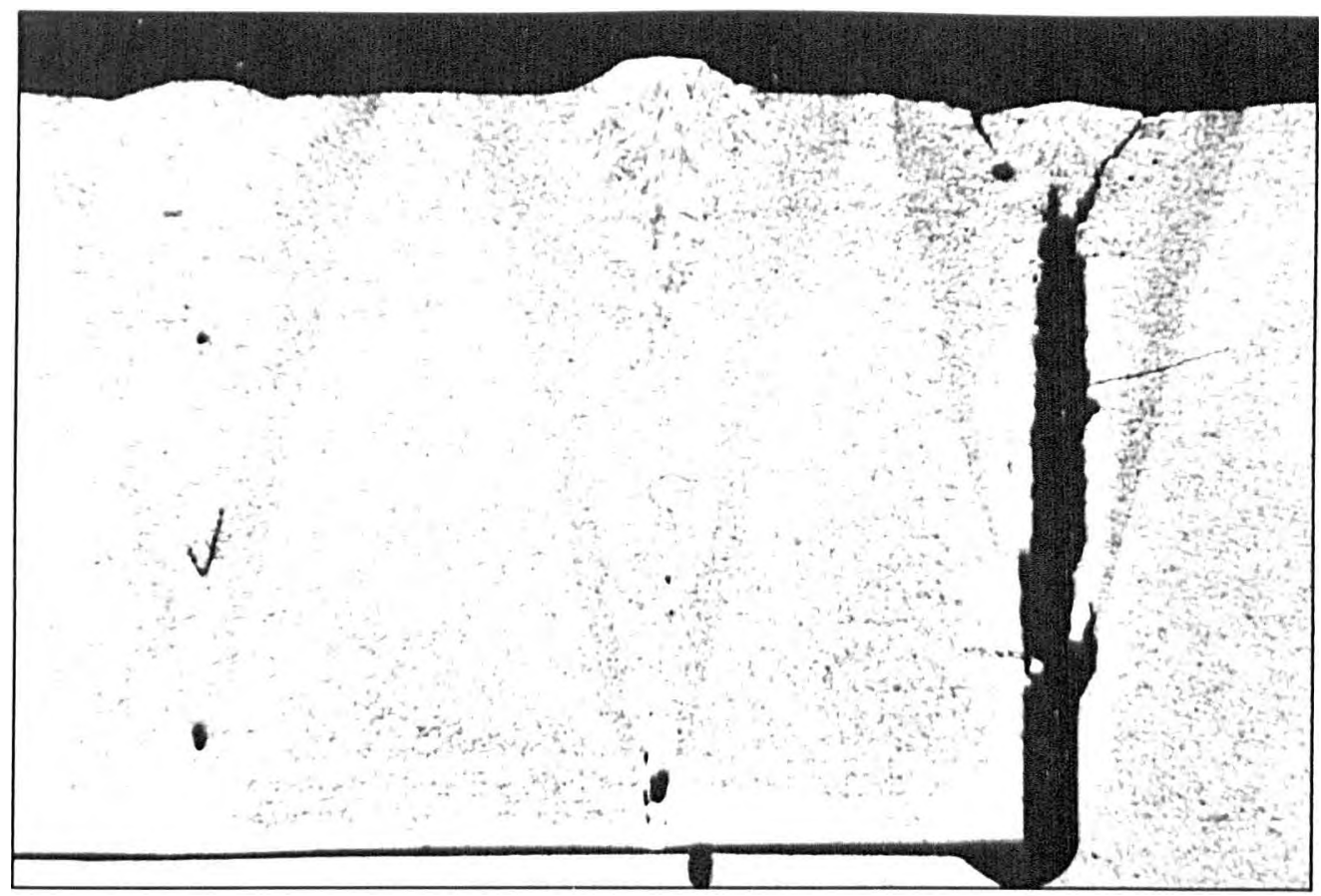

Figure 4.7: Premature failure of the end-cap weld for a tube pressurised with hydrogen at 270 bar and $600^{\prime \prime} \mathrm{C}$.

\section{Internal Specimens and Filler Bars}

To minimise the volume of hydrogen gas, and hence the explosive potential inside the tubes, stainless steel rods were inserted into the tubes before welding the end-caps. In some cases, the inside of the tube was used as a type of autoclave, with small specimens of other materials being exposed to the same temperature and pressure as the tube being tested. Utilisation of this space proved extremely useful for exposure testing.

\section{Tube Preparation}

Initially, the tubes and end-caps were conserved separately with vaseline spray. Each set of endcaps was fitted to a specific tube prior to testing. The joining surfaces between the end-caps and the tube were made as clean as possible by polishing with 800 grit emery paper, and cleaning with acetone to aid in the welding. The inside of the tube was also given a thorough clean with acetone to remove any vaseline. At this point, any internal specimens to be exposed to hydrogen were put into the tube, as well as the filler bars. The tube was then welded shut. After welding, the outside of the tube was polished with 800 grit paper and cleaned with acetone. 


\section{Preliminary Test Results and Subsequent Tempering Treatments}

Initial creep and internal pressure tests of the BS material showed that it was much stronger than expected, with creep lives greatly exceeding previous tests on similar material at the JRC. A comparison of this uniaxial data and the BS material is shown in figure 4.8. Whilst there was no obvious reason for the difference, it was assumed that the initial heat treatment given by the supplier was aimed towards a minimal temper to provide the high strength.

As a knock on effect of the high strength. greater stresses than originally calculated were needed to achieve the desired failure times of the test specimens. In the case of internally pressurised tubes, the maximum stress was limited by the internal gas pressure available, and thus the only way to reach the necessary stress levels was to either reduce the tube wall thickness, or to lower the strength of the tubes. The former option was not considered viable as the wall thickness was designed to be as high as possible to reduce the slope of the hydrogen concentration gradient across it. It was therefore decided to attempt to reduce the material strength by applying a tempering operation.

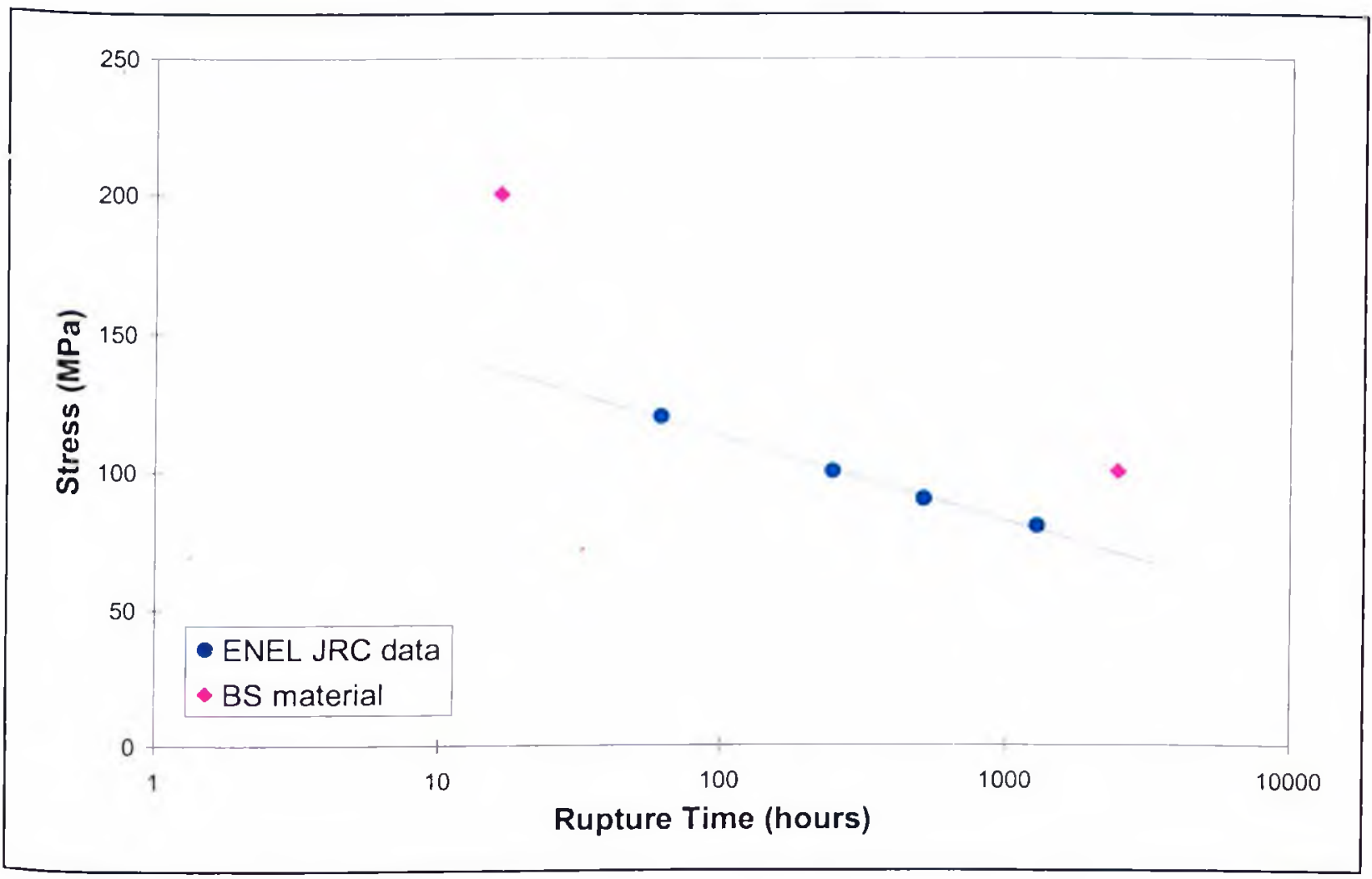

Figure 4.8: Comparison of the $600^{\prime \prime C}$ uniaxial creep rupture strength of the as-received $B S$ material and previously recorded data from the JRC of a normalised and tempered 2.2.5 $\mathrm{Cr}$-1Mo steel 


\section{Initial Tempering Methods}

In order to find the best tempering conditions, several small (approx. $1 \mathrm{~cm}^{3}$ ) specimens were subjected to various times under different temperatures using a highly accurate calibration furnace. The chosen tempering conditions are shown in table 4.3 . Temperatures above $700^{\circ} \mathrm{C}$ were chosen to accelerate the tempering process. Also shown in table 4.3 are the hardnesses of each of these specimens, measured after treatment to ascertain the extent of the tempering. Hardness measurements were made with a Vickers hardness testing machine using a $20 \mathrm{~kg}$ load. The required target hardness was estimated by comparison with another heat of normalised and tempered material available at the JRC, as this steel was known to be much weaker (Figure 4.8), with a hardness of approximately $145 \mathrm{Hv} 20$. Of the various tempering treatments attempted, the chosen one was 4 hours at $750^{\circ} \mathrm{C}$ followed by an air cool, the same as that used in the reference JRC material. However, this did not reduce the hardness to the same level as the reference material, but gave creep strength of the correct order of magnitude when applied to a creep specimen (figure 4.9 ).

\begin{tabular}{|c|c|c|}
\hline $\begin{array}{c}\text { Temperature } \\
\text { ('C) }\end{array}$ & $\begin{array}{c}\text { Time } \\
\text { (hours) }\end{array}$ & $\begin{array}{c}\text { Hardness After } \\
\text { Tempering } \\
\text { (HV20) }\end{array}$ \\
\hline- & - & 205 \\
\hline 725 & 1 & 190.875 \\
\hline 725 & 2.5 & 181.125 \\
\hline 725 & 3.5 & 178 \\
\hline 725 & 4.5 & 174.5 \\
\hline 725 & 6.5 & 170.375 \\
\hline 725 & 8.7 & 167.625 \\
\hline 750 & 1 & 177.375 \\
\hline 750 & 2 & 167.875 \\
\hline 750 & 3 & 165.875 \\
\hline 750 & 4 & 162.5 \\
\hline
\end{tabular}

Table 4.3: Tempering conditions and resulting hardness values of the BS material

\section{Tempering of Pre-Machined Tubular Specimens}

Once the desired tempering conditions had been decided, it was necessary to apply these conditions to the tubular test specimens that had already been machined. However, several technological problems had to be overcome. In order to prevent massive oxidation of the specimens under the high tempering temperatures, an inert environment was required. In addition, the high mass and length of the tubes meant that it was difficult to provide an even 


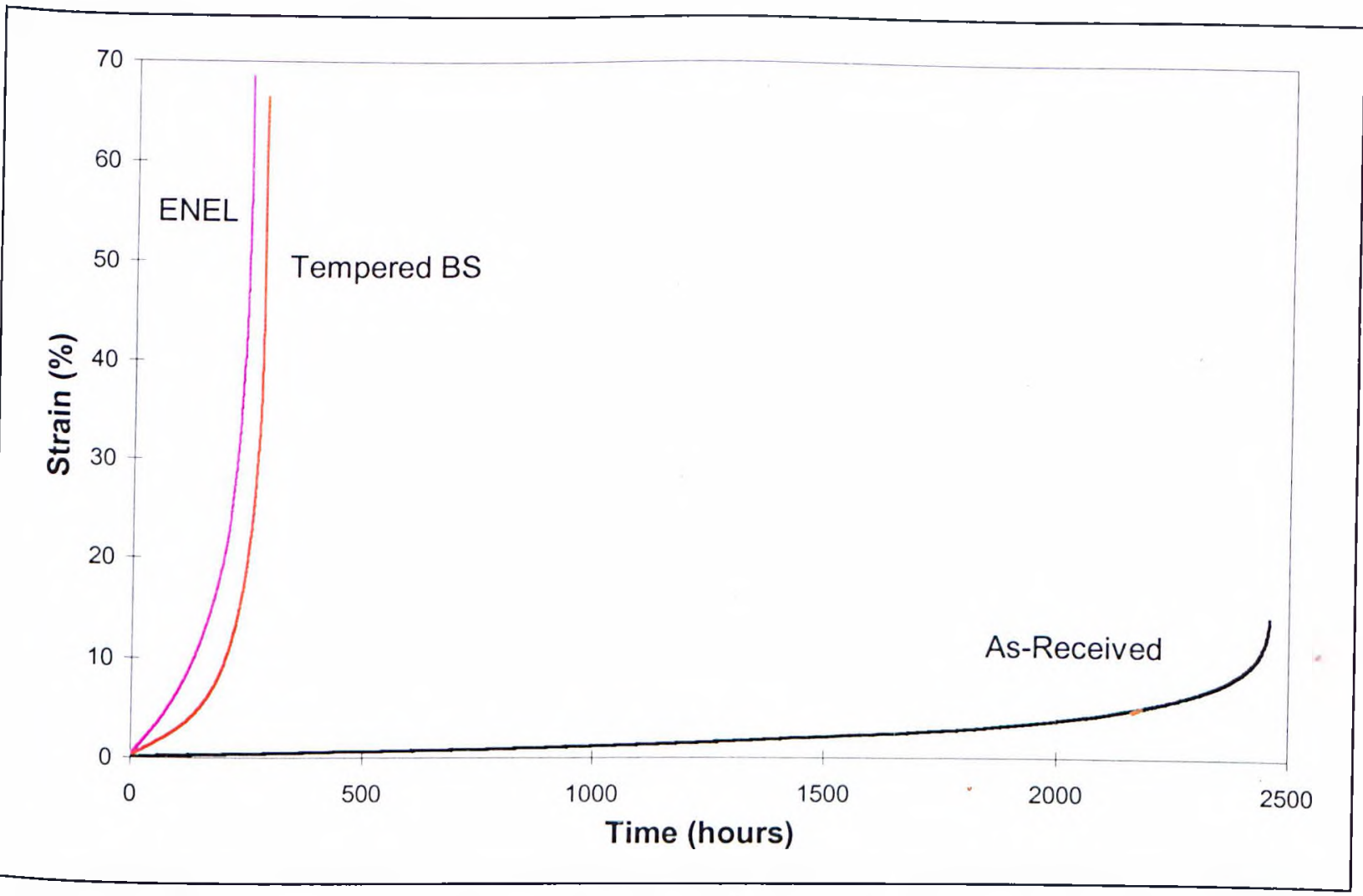

Figure 4.9: Comparison of the uniaxial creep curves for the BS material before and after tempering (4hrs, $750^{\circ} \mathrm{C}$ ) with the ENEL JRC data. All curves carried out under 100MPa stress and $600^{\circ} \mathrm{C}$

temperature gradient across them. A high heat input was therefore required to bring the temperature directly to the desired value so that a clear tempering start time could be defined.

After much experimentation with various set-ups and dummy specimens, a tempering furnace rig was decided upon which gave consistent and well defined thermal cycles for all of the specimens. The set-up used is shown in figure 4.10. Before the initial heating of the specimen, a high power vacuum pump was used to evacuate the heating chamber to a pressure of approximately $0.00 \mathrm{l}$ bar. The chamber was then flushed with argon and the procedure repeated twice more. A small flow of slightly over-pressure argon within the furnace tube ensured that an inert atmosphere was maintained around the specimen during the tempering procedure.

Heat was supplied by a 5000 watt single zone Carbolite furnace, used in conjunction with a Eurotherm temperature controller. Insulating buffers placed either side of the specimen reduced the temperature gradient from the centre to the edges to only three degrees. A pre-heating procedure at $250^{\circ} \mathrm{C}$ for about 2 hours was found to provide greater stability in the following heat up stage to $750^{\circ} \mathrm{C}$. Three $\mathrm{N}$ type thermocouples were used to measure the temperature of the specimen in the centre and the extremes of the gauge length. After four hours at $750^{\circ} \mathrm{C}$, the 
furnace was turned off and the specimen was moved out of the hot zone with the push rod shown in figure 4.10 to provide a cooling rate more closely approximating that of an air cool. A typical thermal cycle is shown in figure 4.11 for the temperature in the middle and at the extremes of the gauge length.

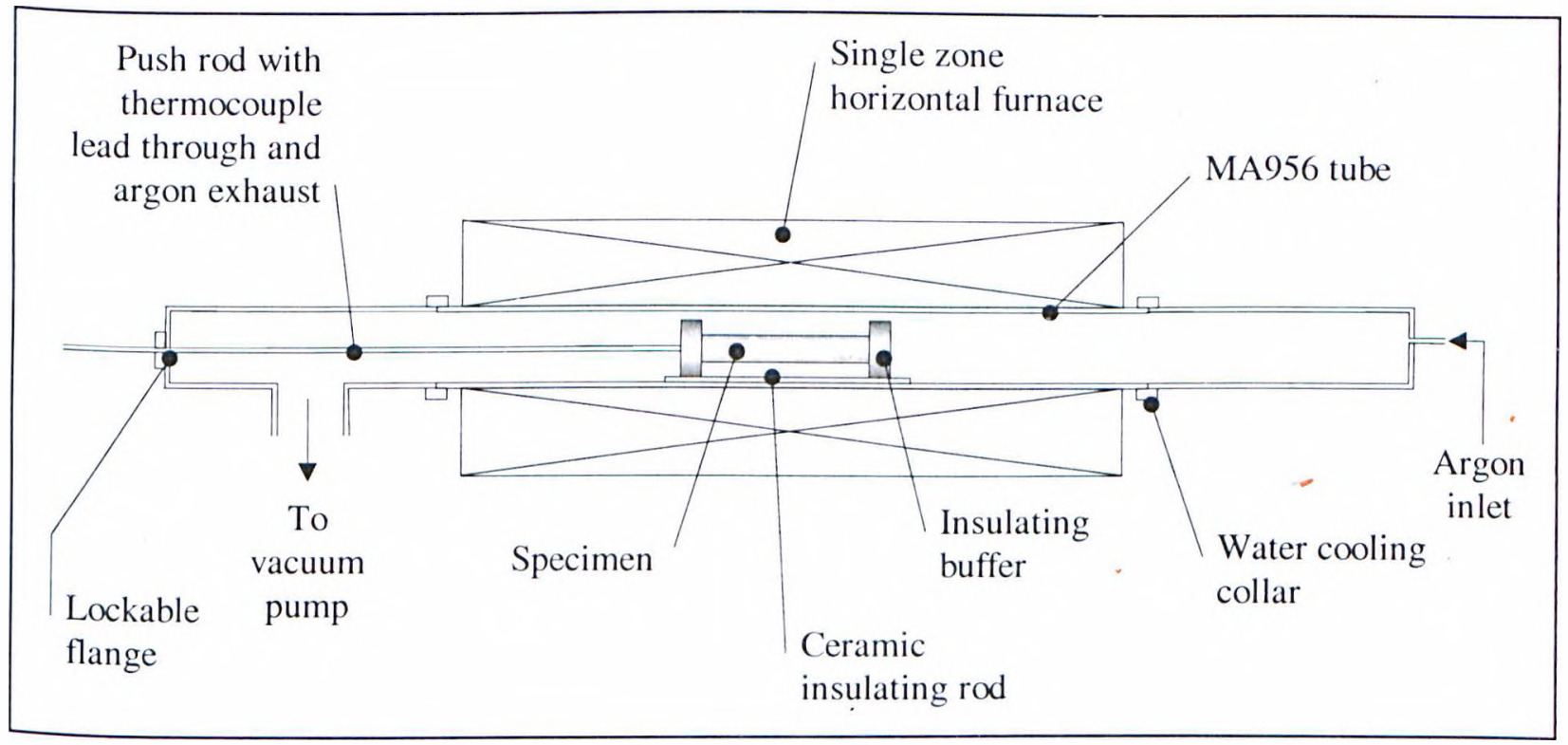

Figure 4.10: Schematic illustration of the rig used for tempering the pre-machined tubular specimens in an inert environment

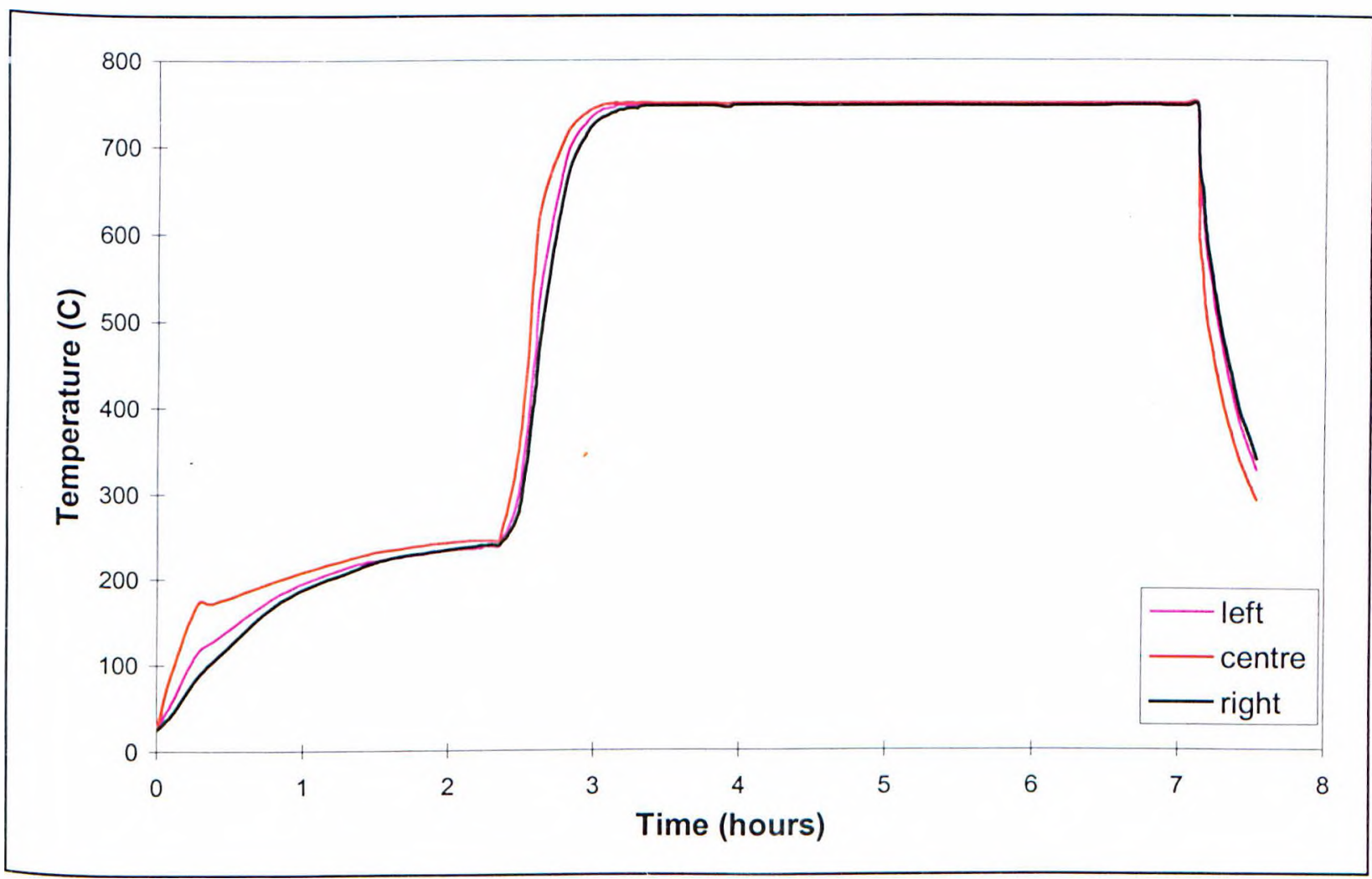

Figure 4.11: Typical thermal cycle of a tube undergoing a tempering operation at $750^{\circ} \mathrm{C}$ for 4 hours 
To check the extent of tempering, hardness tests were performed on the shoulders of the specimen at either end. In addition, small pieces of the BS material had been inserted into the tubes prior to tempering, such that an indication of the hardness could be measured at various positions along the gauge length. The hardness values of the specimen shoulders were consistently reduced to value of approximately $165 \mathrm{Hv} 20$, with the internal gauge length specimens correlating very well.

\section{Tempering of Uniaxial Specimens:}

Having already used several of the uniaxial creep specimens, there was some concern that there were not enough left to give a complete data set. Other specimens would then have to be machined and tempered separately which could lead to small differences in heat treatement, It was therefore decided to re-make a new batch of uniaxial specimens which could all be garunteed to have the same heat treatment. Using the same procedures as above, a rough-cut section of the BS as received pipe measuring approximately $250 \mathrm{~mm}$ in length was tempered in the furnace. After tempering, this piece was used for the machining of new uniaxial creep specimens so that all the uniaxial specimens had the same treatment, and importantly, that both uniaxial and multiaxial test specimens had the same properties.

\section{Re-Characterisation of Material After Tempering}

The microstructure of the BS material after tempering is shown in figure 4.12. There appears to be no change in grain size when compared to figure 4.1, although the structure seems to be

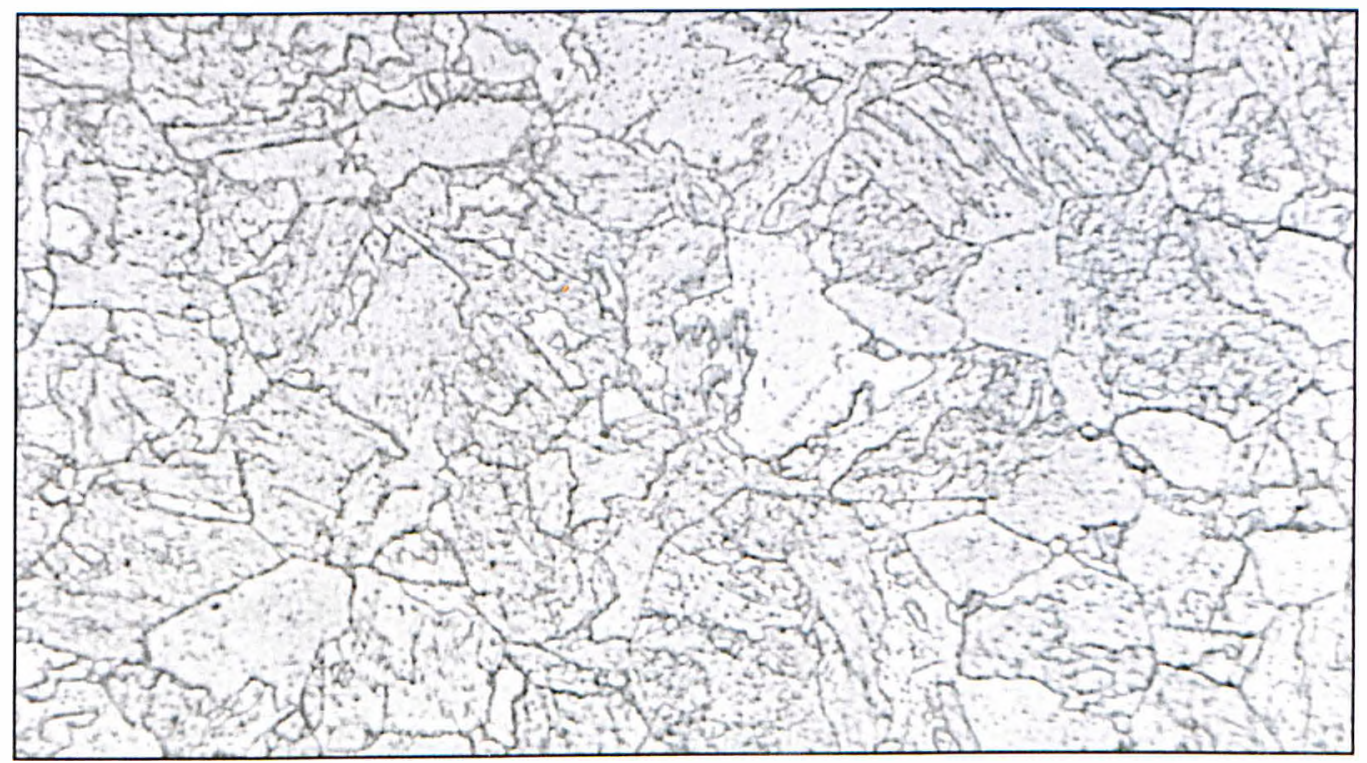

Figure 4.12: Optical micrograph of the BS steel after a tempering treatment of $750^{\circ} \mathrm{C}$ for 4hrs. (magnification: $x 400$, etched in $5 \%$ Nital) 
more uniform after tempering. Carbide extraction replicas (figure 4.13) show that tempering has caused coarsening of the carbide particles, with virtually all the small needle shaped carbides being removed after the tempering operation. Based on previous experience at the JRC, these carbides are assumed to be $\mathrm{M}_{2} \mathrm{C}$ type, probably containing a high quantity of Mo. It is then obvious that the creep strength decreases after tempering due to the increased size and spacing of the carbides, and in particular the elimination of $\mathrm{Mo}_{2} \mathrm{C}$ which is well known for its ability to confer high creep strength.

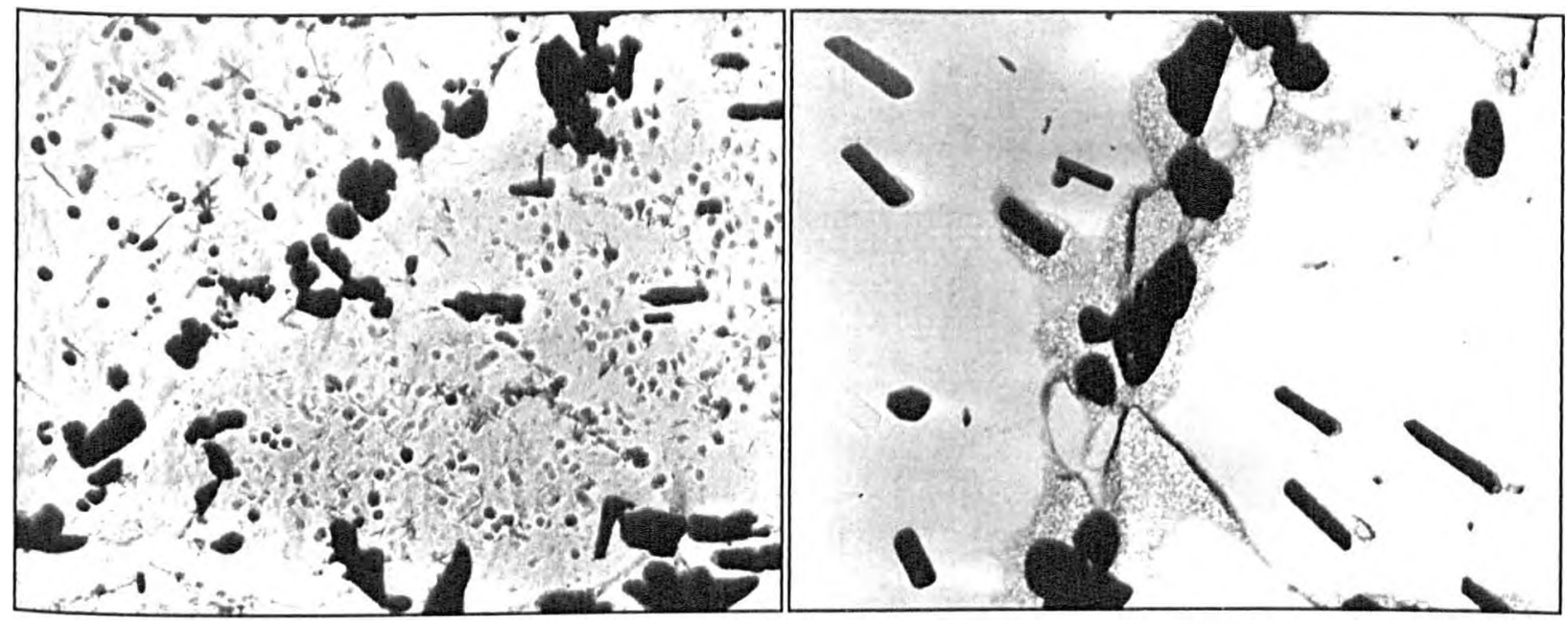

a)

b)

Figure 4.13: Carbide extraction replicas showing the difference in carbides a) before and b) after tempering the BS material (Mag. x8000.) 


\subsection{Uniaxial Creep Testing in Air}

In order to provide a basic data-set against which the creep tests in hydrogen and the multiaxial tube tests could be compared, it was also necessary to perform creep tests in an air or inert environment. Creep testing under an inert environment would perhaps be the ideal situation, as it eliminates the factor of specimen oxidation, which can influence the lifetime when the specimen diameter is small. Whilst the creep rigs for use in a hydrogen environment (see section 4.3) are capable of performing creep tests in both low and high pressure argon as well as hydrogen, the time requirements meant that it was more efficient to carry out the uniaxial creep tests in standard rigs, in air. It is generally assumed in this thesis that the influence of oxidation on the specimen lifetime is relatively small. The following section describes briefly the testing set-up and procedures used for carrying out creep tests in air.

\section{The Uniaxial Creep Rigs}

\section{Loading Method}

The rigs used for testing were MAND ${ }^{\mathrm{TM}}$ constant load, dead weight lever arm machines as shown schematically in figure 4.14. Specimen loading was applied through a 10:1 lever arm ratio, which is kept constant by maintaining the lever in a horizontal position throughout the test. As the specimen strains the loading weights move downwards until they trip a switch which causes the motor to turn, thereby lifting the weights a small amount and maintaining the lever position. On failure the weights trip a further switch, which automatically turns off the furnace. Calibration of the loading lever was obtained with a Rein ${ }^{\mathrm{TM}}$ proving ring.

\section{Heating Method}

Specimen heating was applied with a three zone 3 kilowatt Mike Lockwood ${ }^{T M}$ furnace. Three ' $S$ ' type control thermocouples were connected to Eurotherm ${ }^{T M}$ temperature controllers. The specimen temperature was measured with three ' $\mathrm{K}$ ' type thermocouples, positioned such that one touched the middle of the gauge length, and the other two were in contact with the specimen shoulders. Normal temperature control of $\pm 0.5^{\circ} \mathrm{C}$ was obtained at $600^{\circ} \mathrm{C}$. This was only obtained by careful insulation at the top and bottom of the furnace. To ensure accuracy, the thermocouples were replaced after about 1500hours testing. In addition, each new batch of thermocouples was checked in a highly accurate calibration furnace prior to use. 


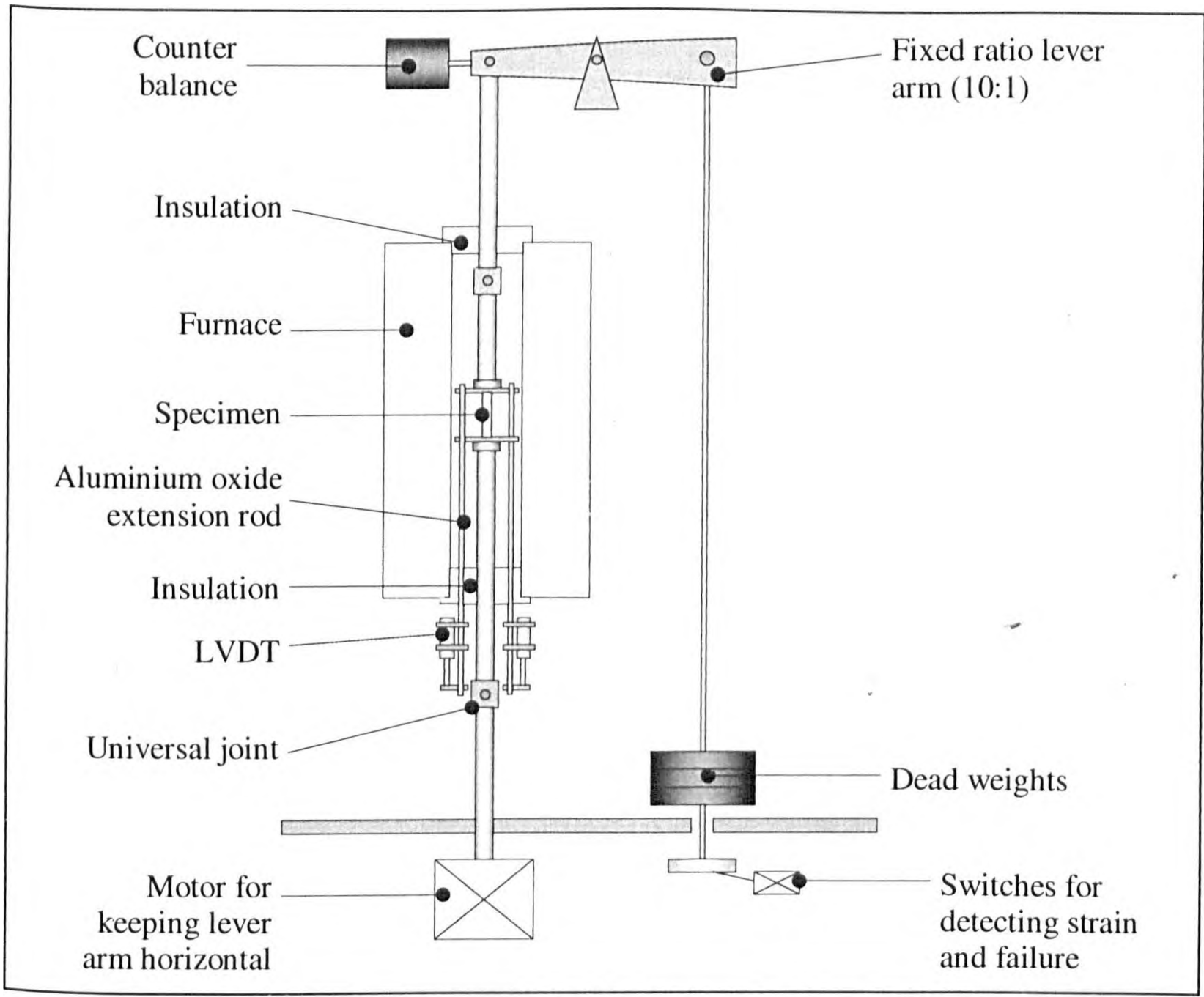

Figure 4.14: Schematic of the creep rig for uniaxial creep testing in air

\section{Measurement of Specimen Elongation}

Aluminium oxide extension rods were connected to the top and bottom of the specimen gauge length by means of grips that were machined to fit the specimen knife-edges. These rods were led out of the bottom of the furnace via holes cut into the insulation material. LVDTs with a $50 \mathrm{~mm}$ range were used to measure the relative displacement between those rods connected to the top and the bottom of the specimen. The use of two LVDTs allows averaging of their values, which gives more accurate strain results. The LVDTs were regularly calibrated against high accuracy, ceramic spacer blocks. A typical calibration is shown in figure 4.15. 


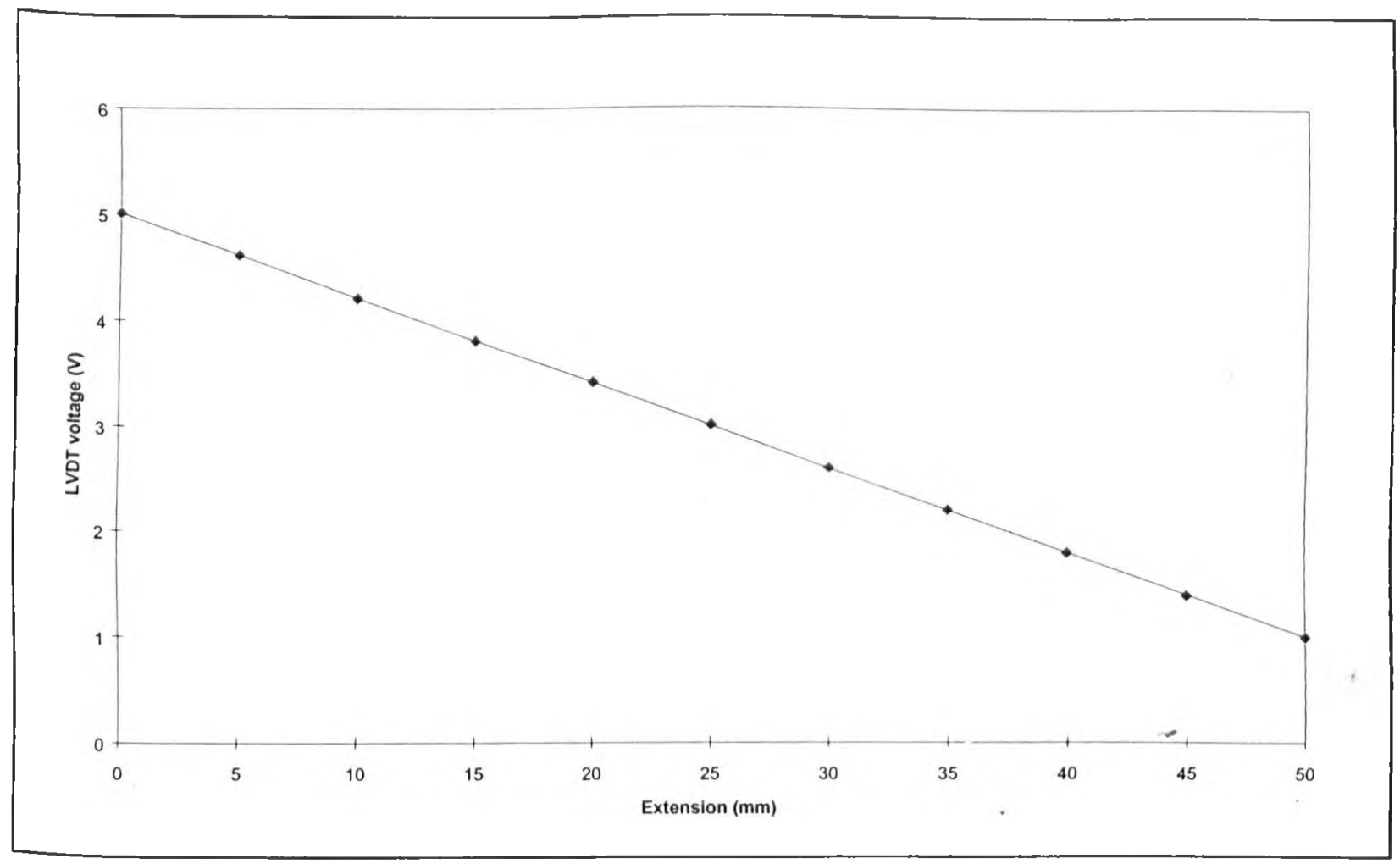

Figure 4.15: Typical LVDT calibration of extension vs output voltage for uniaxial creep testing

\section{Testing Procedures}

\section{Specimen Mounting}

Before mounting, the specimen gauge diameter was measured with a micrometer in five different positions so that the average cross sectional area could be calculated. Next, the extension arms were attached to the specimen, followed by the thermocouples. Finally the specimen was screwed in to the load train and mounted in the rig, ensuring that the lever arm was horizontal and that the specimen was in the middle of the furnace. A small $20 \mathrm{~N}$ load was applied to the specimen to take up any slack in the system.

\section{Specimen Heating}

Temperature set-points were initially set at about $500^{\circ} \mathrm{C}$. The motor on the rig was set to automatic to ensure that movements caused by thermal expansion were accounted for. Once stable, the temperature controllers were then adjusted independently until the test temperature of $600^{\circ} \mathrm{C}$ was reached. It was normally necessary to leave the rig overnight to allow the temperatures to settle. 


\section{Specimen Loading}

When the specimen temperature had stabilised, the load could be applied. This was done by first lowering the loading pan until it rested on an aluminium spacer. The weights could then be applied without the risk of shock loading the specimen. Once the correct amount of load was added for the required stress (gravity taken as 9.81), the motor was turned on so that the weights were slowly lifted away from the aluminium spacer. The motor was then set to automatic and the test start time recorded.

\section{Data logging}

Both the LVDT voltage signals and the temperature were logged on a Dell ${ }^{\mathrm{MM}}$ computer with a data acquisition programme written in-house with the Labview ${ }^{\mathrm{TM}}$ programming language. In addition, the programme converted the LVDT voltages into a strain measurement, and wrote all the data to file for later manipulation. Data was normally saved to file every half-hour, or every hour for longer-term tests. A log book was also kept and updated daily for manual data recording. 


\subsection{Uniaxial Creep Testing in Hydrogen}

Two identical uniaxially loaded creep testing rigs for use under high-pressure hydrogen have been designed and built. The rigs were designed to be capable of measuring creep strain under working conditions of more than $22 \mathrm{MPa}$ hydrogen pressure at temperatures over $600^{\circ} \mathrm{C}$ and loads of up to $10 \mathrm{KN}$. An overall schematic of the rigs and a photograph can be seen in figures 4.16 and 4.17 respectively. The design, fabrication and employment of such rigs requires much technological innovation and thought, especially in terms of safety. Due to the severe working conditions, there are very few laboratories in the world capable of building similar rigs, and as such, there are very few guidelines for their construction. The following section aims to describe the design and function of these rigs. For simplicity, from this point onwards, the rigs will be referred to as the hydrogen creep rigs (HCR).

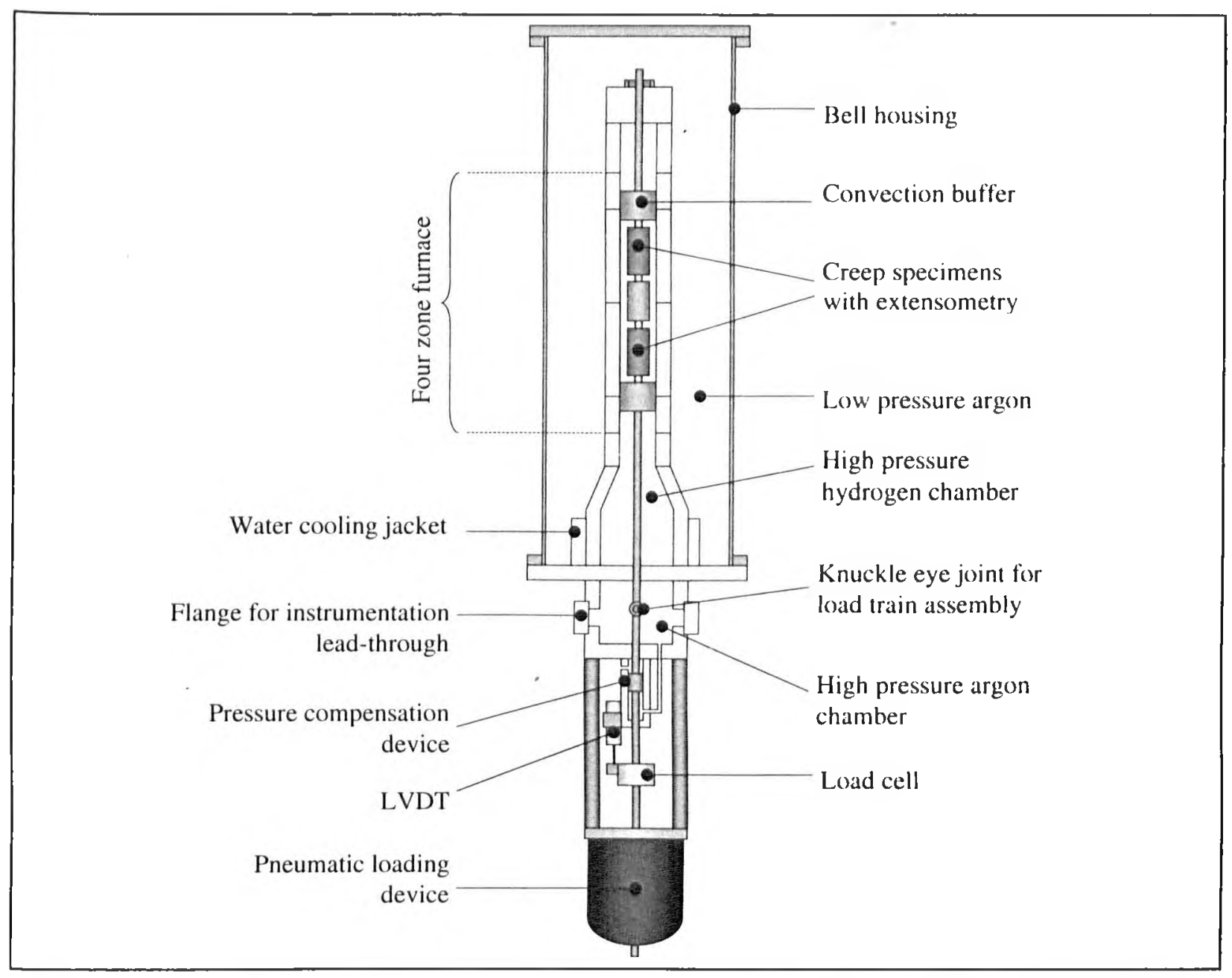

Figure 4.16: Schematic Illustration of the creep rig used for uniaxial loading under highpressure hydrogen environments 


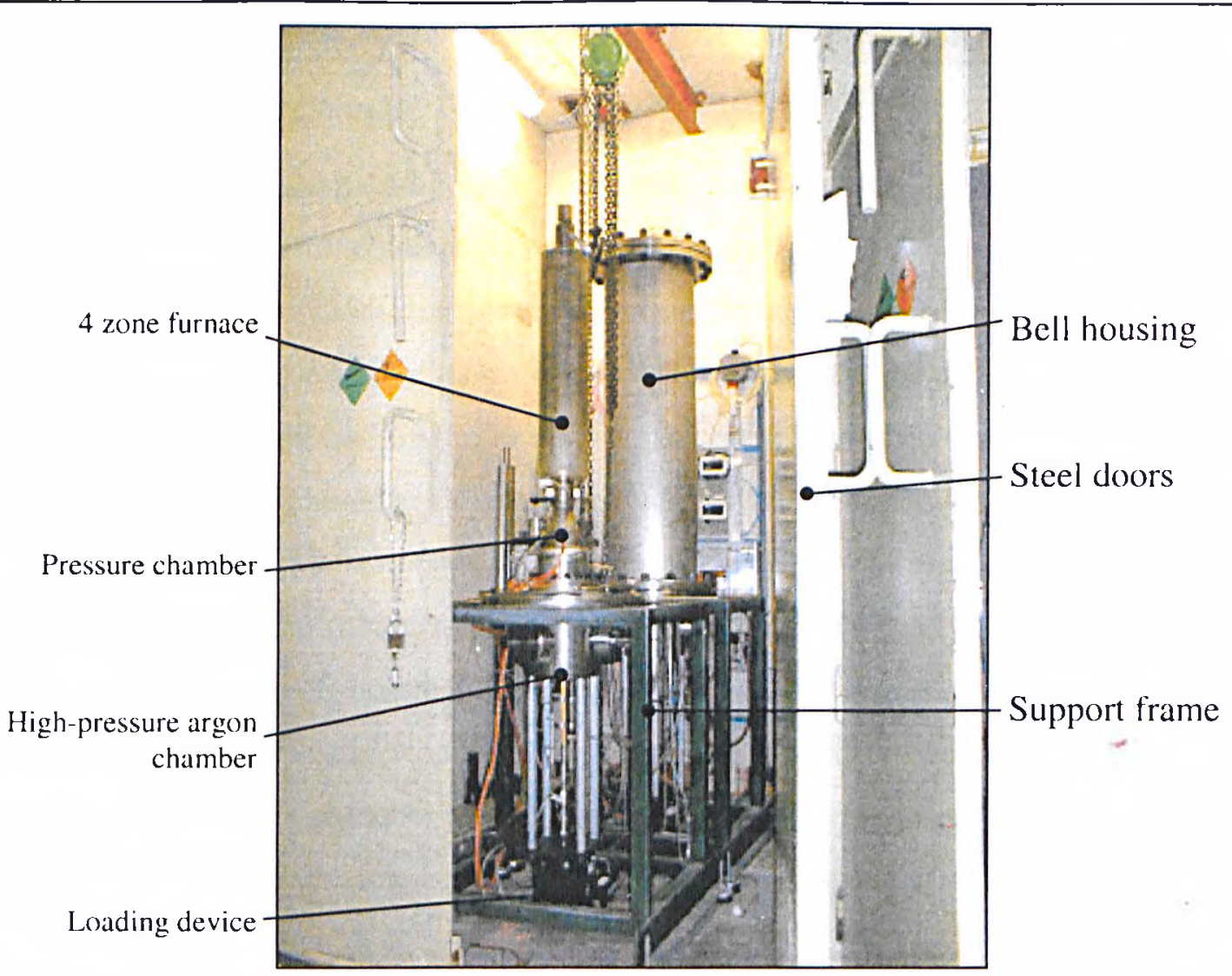

Figure 4.17: Photograph of the HCR rigs in a concrete cell with steel doors. The rig at the back has the bell housing on, and the rig nearest the doors is shown without the bell housing

\section{Other Project Influences on the Rig Design}

In addition to the work of this thesis, there were other driving forces for the creation of the hydrogen creep rigs. The most important of these was the Brite Euram PREDICH project, which had some input into the design of the rigs. One of these inputs was to make the rigs capable of loading two specimens in series so that a greater volume of test data could be obtained in a given time. Both specimens would be under the same load until rapid straining in one of them lead to failure. The other specimen would then be in a critically damaged state, making it ideal for metallographic investigations into the damage processes. Another input on the design was the specimen size and dimensions agreed upon by the PREDICH partners, having a gauge length of $50 \mathrm{~mm}$ and a gauge diameter of $8 \mathrm{~mm}$. These dimensions were somewhat larger than those used in the current work, so it was necessary to build small adapters to allow the BS material specimens to fit into the HCR load train. 


\section{Active Gas Containment and Safety in the HCR}

\section{Hydrogen Gas Pressure Chamber}

The pressure chamber containing the specimens, extensometry gauges and load train was designed to withstand a total internal pressure of over $220 \mathrm{bar}$ at temperatures above $600^{\circ} \mathrm{C}$. Type 316 stainless steel was chosen for construction because of its' availability, weldability and strength under hydrogen environments. The pressure chamber was also designed to withstand any compressive force applied by the load train, since the load train was anchored to the top of the pressure chamber. The internal diameter was made as small as possible (allowing for accommodation of the extensometers and the load train) to minimise the volume of hydrogen gas present. Water cooled rubber o-rings were used to seal the load train at the point where it passed out of the bottom of the pressure vessel, and a swaged type stainless steel seal prevented leakage at the top of the vessel where the load train was bolted on. Separate active gas inlet and outlets were built into the chamber to allow greater control of the internal pressure, and to allow a small flow past the specimens during a test. This ensured continuity of the hydrogen environment.

\section{Safety Blanket Gas}

Just under the hydrogen pressure chamber was another smaller chamber filled with slightly over test-pressure argon. This provided a low-pressure gradient across the rubber o-rings around the load train, which was intended to lower the risk of hydrogen leakage past those rings. In addition, any hydrogen that did leak was then in an inert environment where it could not react explosively with oxygen.

Taking this principle further, the whole pressure chamber area was surrounded by a large steel bell housing which was filled with low-pressure argon. The oxygen and hydrogen content of this argon was constantly monitored so that any leak could be detected, and the risk of subsequent explosion was minimised. In addition, a constant flow of argon was maintained through the bell housing, thereby flushing away any potential build up of hydrogen or oxygen. The volume of the housing was large enough so that a catastrophic leak from the pressure chamber could be contained without the risk bursting.

\section{Pressure Compensation on the Load Train}

The high pressures used in the gas chamber induce an axial load on the load train, which must be considered in load calculations. To compensate for this an equalising device was 


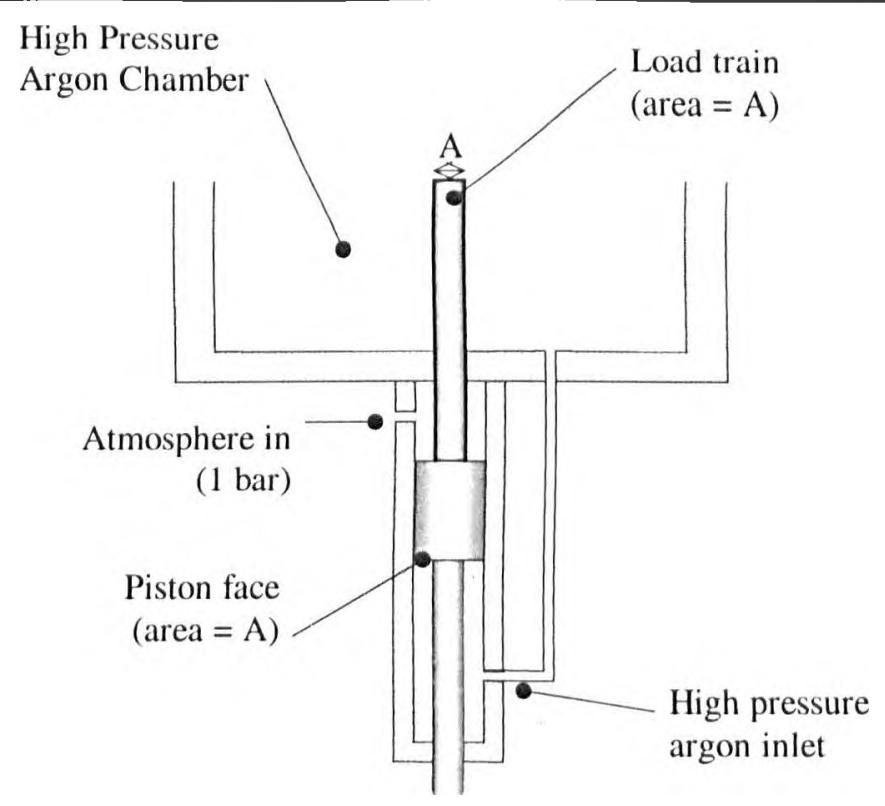

Figure 4.18: Schematic of the pressure compensation device used to prevent additional axial loading due to the internal test pressure

constructed, based on an original idea used in a similar Japanese rig [Yokoga.'78]. The same device had also been used successfully on the load train of a compact tension specimen testing rig previously constructed at the JRC [Looney'94], and is shown in figure 4.18. Basically, the device is a piston which is fed from the high-pressure argon chamber. The action of the piston is designed to work against, and exactly balance the internal test pressure. This is achieved by making the surface area of the piston head equal to the cross sectional area of the load train as shown in the figure.

\section{Gas Supplv Infrastructure and Safety}

The high pressure hydrogen required for the tests is provided by a diaphragm compressor, capable of pressurising hydrogen to over 270bar. The compressor is supplied by a rack of two 50 litre, 200bar bottles which are connected via hand operated pressure regulators and high pressure pipeline, capable of supporting up to 4000bar. Pressure relief burst valves are incorporated into this system in case of accidental over pressurisation. Both the compressor and the bottles are stored in a separate, well ventilated garage outside the main laboratory area. All rigs for use under high pressure are situated within concrete cells with steel doors, which are designed to stop debris in the event of an explosion. The high pressure gas is pumped into the rigs via a series of manual and automatic valves, the layout of which are shown in the control panel for the rig (figure 4.19). The hydrogen pressure is controlled by the high pressure argon 


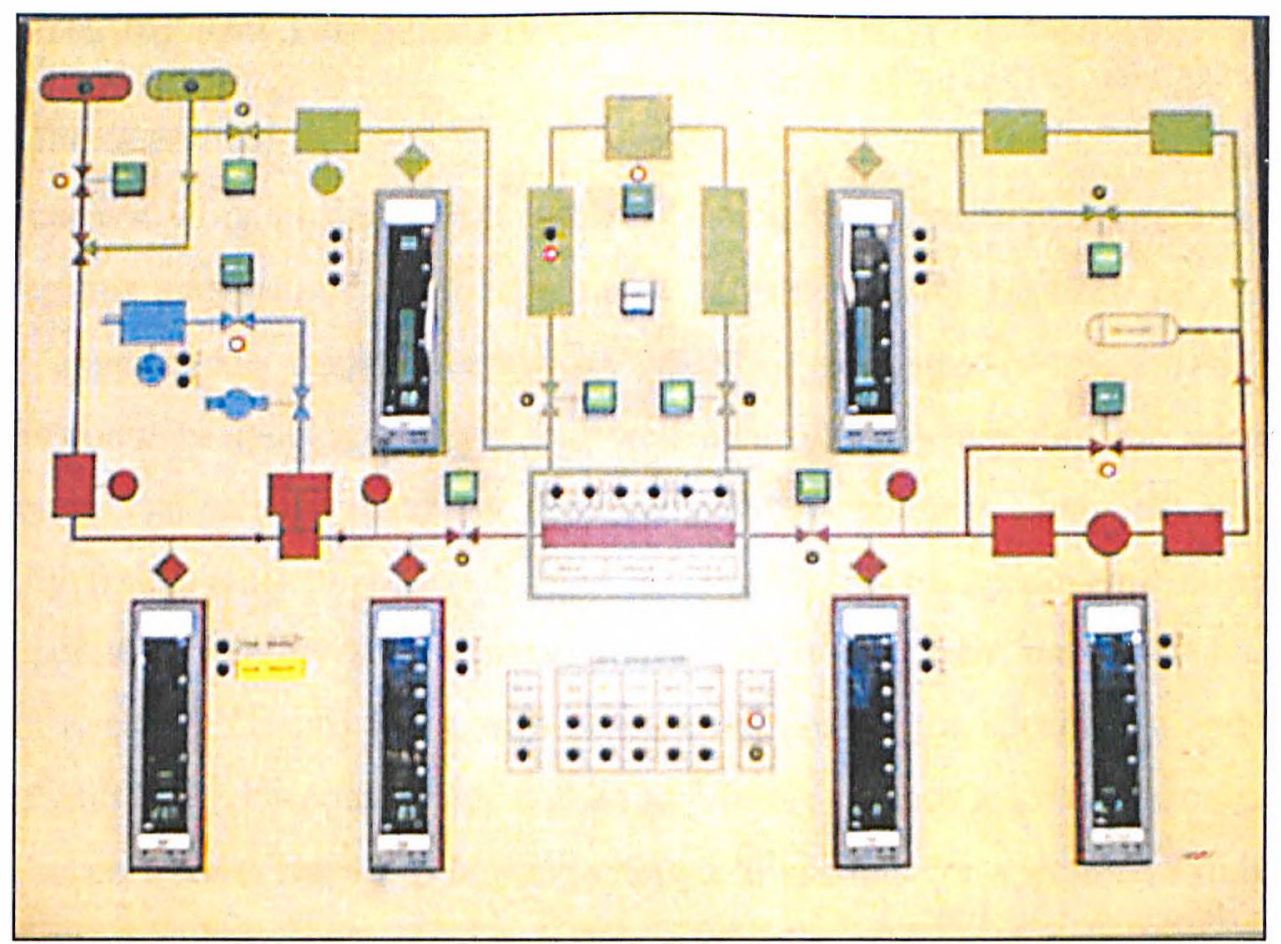

Figure 4.19: Control panel for the hydrogen uniaxial creep rigs in, showing the layout of gas supply lines and valves

which provides the low pressure differential seal at the bottom of the exposure chamber. Thus, if the argon supply is cut off for any reason, the hydrogen will also be cut off.

The argon blanket gas is fed through both oxygen and hydrogen monitors. Pre-set alarm levels of these gasses allow the active gas to be dumped through the exhaust valve, and the furnace to be automatically turned off in the event of hydrogen leakage or oxygen contamination of the bell housing. In addition, hydrogen detectors are placed in the compressor garage, the concrete test cells and the control room. All of these are connected to alarms which automatically stop the test and dump the pressurised gas if dangerously high hydrogen levels are detected.

As an additional safety feature, the hydrogen gas supply can be switched to helium for leak testing purposes. A highly sensitive "Leybold UL100 Plus" helium detector is used to check the gas system for possible leaks, especially at joints and seals before hydrogen pressurisation. Extra checks are also carried out with "Snoop"TM soap solution, which bubbles where a leak is present. 


\section{Specimen Heating and Temperature Control in the HCR}

\section{Specimen Heating Method}

Whilst the current work in this thesis is based around creep tests performed at $600^{\circ} \mathrm{C}$, the PREDICH testing programme required a range of temperatures between 575 and $610^{\circ} \mathrm{C}$. Preliminary experiments using Thermocoax sheathed resistance wire showed that these temperatures could be quickly reached and well controlled by winding the wire into spiral grooves around the pressure chamber. The temperature gradient along the specimen was minimised by fixing anti-convection buffers either side of the specimen. To provide even greater temperature stability and control, the furnace was split into four heating zones consisting of two long $(16 \mathrm{~cm})$ zones with $2 \mathrm{~kW}$ power around the specimens, and two shorter $(8 \mathrm{~cm})$ zones with $1 \mathrm{~kW}$ power at the top and bottom of the furnace to control thermal gradients. The longer zones were designed to be long enough to account for specimen extension during creep, and thus were slightly offset towards the top of the specimens. Finally, heat loss from the thermocoax wire was limited by surrounding the four heating zones with Thermiculite insulation. A schematic illustration of the completed furnace is shown in figure 4.20.

\section{Temperature Measurement and Control}

Four sheathed 'S' type thermocouples were attached directly to the centre of the four heating zones. These thermocouples provided the input into four Phillips PID controllers which could

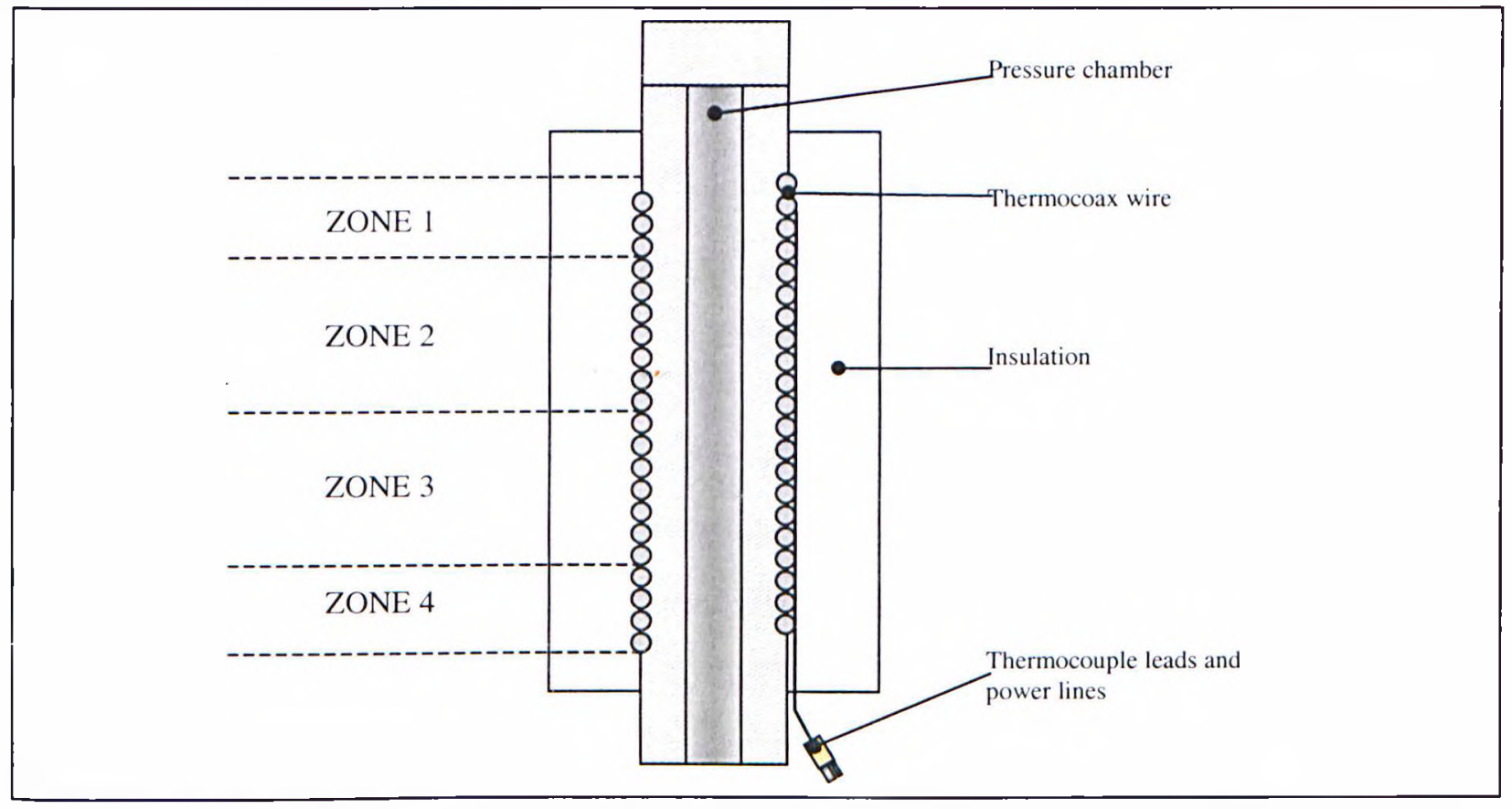

Figure 4.20: Schematic illustration of the four zone Thermocoax furnace used in the HCR. 
be given independent set points. The specimen temperatures were measured with two sheathed ' $N$ ' type thermocouples positioned at the top and bottom shoulder part of each specimen. It was not always possible to measure the temperature of the specimen gauge length directly because of the extensometry design (see later), however, previous experiments had shown that the position of the thermocouples on the shoulder sections gave a good representation of the true gauge temperature. Moreover, it was found that the temperature gradient across the length of the specimen could be controlled within $1^{0} \mathrm{C}$ or less.

\section{Creep Specimen Extensometry}

\section{The Design Problem}

Traditional methods of strain measurement in creep involve the use of extension arms connected to the top and bottom of the specimen gauge length. These arms lead from the specimen so that the displacement between those arms connected to the top, and those connected to the bottom of the specimen can be measured using an LVDT. However, when high pressure gas is being used, the arms would have to pass through some sort of seal which would induce friction and hence uncertainty in the strain readings. An alternative method is to measure the displacement of the load train somewhere outside of the furnace. This technique cannot be used though when two specimens are being tested in series, as the strain rate of each may differ. To overcome these problems, an in situ capacitive displacement transducer was decided upon, the workings of which are described below.

\section{Capacitance Displacement Transducers}

A set of displacement transducers were designed and made in conjunction with SJB Engineering Ltd., Bristol. A schematic illustration of the design is shown in figure 4.21. These transducers have the advantage of small size, ability to be used in situ, and high accuracy. Two non contacting concentric cylinders are connected to ridges machined on the specimen by locking tapered fits. The tapers are designed such that gravity ensures the lock remains tight throughout the creep test. The inner cylinder is earthed through the specimen, whilst the outer is isolated from it by a ceramic spacer, and thus a capacitor is created by the area of overlap between the two cylinders. As the specimen strains, the inner cylinder is pulled out from the outer cylinder so that the area of overlap decreases. The resulting change in capacitance is then converted to a voltage which is calibrated against the displacement. A typical calibration against highly accurate ceramic spacer blocks is carried out using a split specimen, the results 


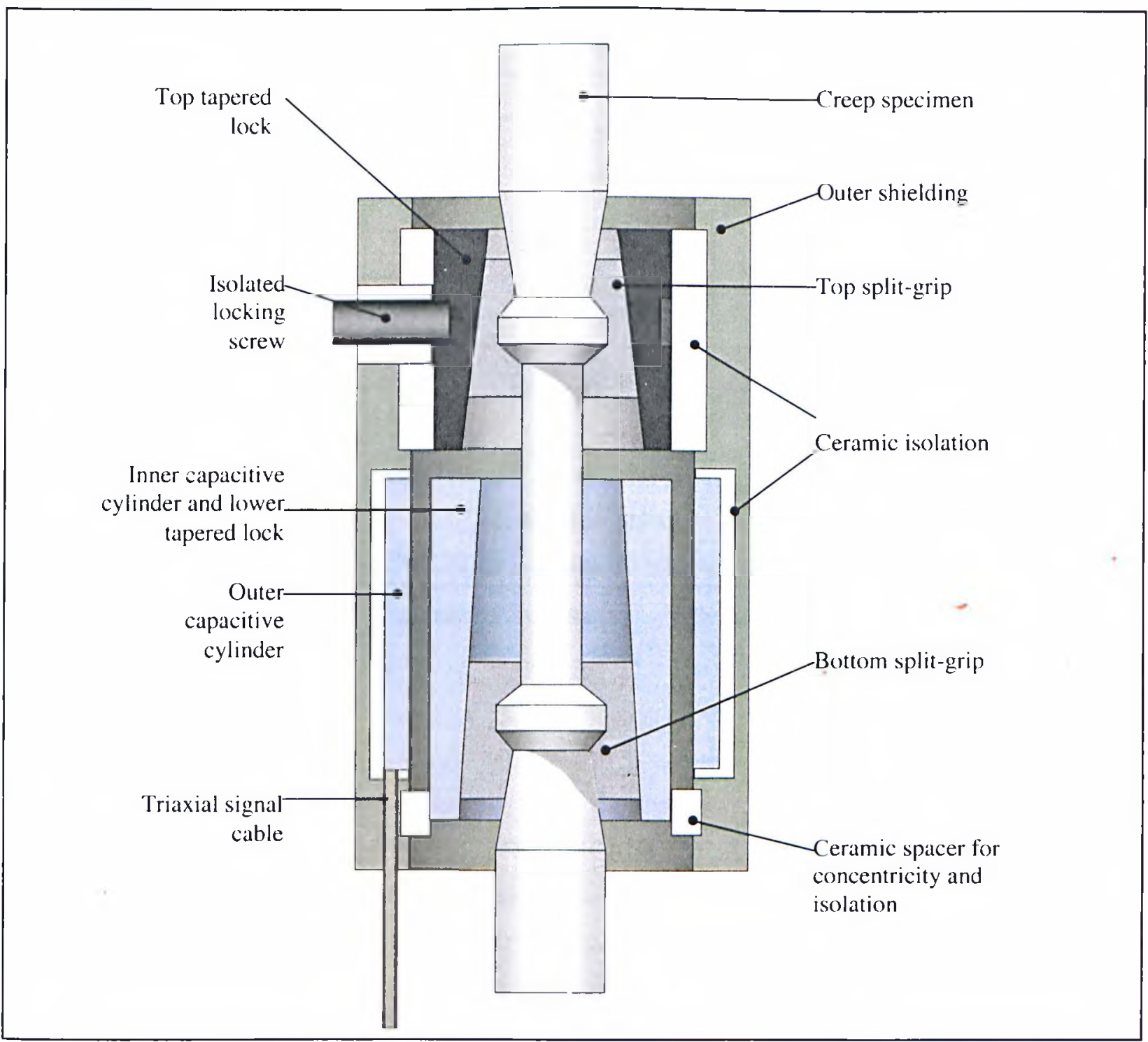

Figure 4.21: Schematic of the capacitive extension gauge for uniaxial creep specimens

of which are shown in figure 4.22. For added resistance to noise, the outer cylinder is sheathed and the signal is carried from the transducer using a triaxial cable. Two types of transducer were produced to fit the PREDICH and the BS specimens.

Several problems were encountered during the development of the capacitive gauges, particularly when operating under high temperatures. These were generally associated with concentricity of the specimen and electrical shorting between either the two halves of the capacitor, or one part of the capacitor with the shielding. Whilst development was ongoing, there was not enough time to carry out any verification tests. It was therefore decided to carry out the majority of creep tests with single specimens (not two specimens in series in the load train), so that displacement could be measured with an LVDT attached to the load train. 


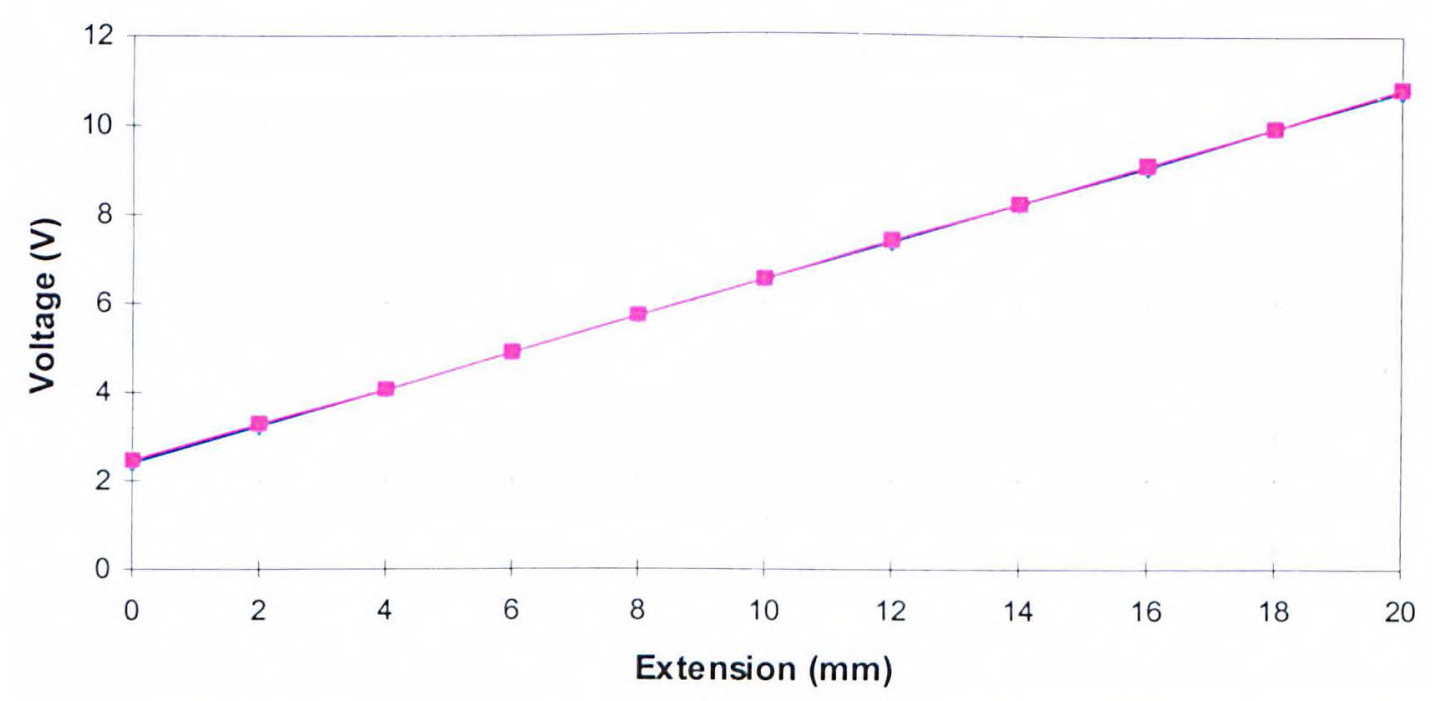

Figure 4.22: Typical room temperature calibration of the uniaxial capacitive extensometry

\section{Creep Specimen Loading}

\section{The Loading Device}

Based on both the PREDICH and thesis requirements, it was decided that a maximum load of $10 \mathrm{KN}$ would be sufficient for all envisioned tests. Having had good experience with pneumatic loading devices in previous rigs [Looney'94], a two way (push/pull) pneumatic device was chosen for the PREDICH rigs. The main advantage of such a system is the small size and elimination of the need for difficult dead weight loading procedures. The chosen device had a maximum free movement of $100 \mathrm{~mm}$, more than enough for the expected combined strains of two specimens in series. A HBM load cell, calibrated with a Rein proving ring was used to measure the applied load, and Coreci PID controllers meant that the load was controllable to within approximately $\pm 5 \mathrm{~N}$.

\section{The Load Train}

The load train was constructed from $16 \mathrm{~mm}$ thick stainless steel. Rubber o-ring type seals were used at all junctions with pressure chambers, whether argon or hydrogen. The load train was made long enough to account for the expected strain of the two samples, and long enough beneath the furnace to help cooling before passing through the first set of seals. A knuckle eye joint was used within the high pressure argon chamber to provide a means of assembling the load train and to provide some flexibility. In addition, the seals through to the hydrogen chamber were designed to accommodate some lateral movement. 


\section{Frictional Load Effect of the O-rings}

It was suspected that the load applied to the specimen would be slightly lower than that given by the load cell due to the frictional force exerted on the load train by the many o-rings. This effect becomes greater under high pressures when the o-rings are compressed. To calibrate this, a high strength spring was connected to one of the capacitance gauges and mounted in the load train. Previous calibrations of the spring meant that it was possible to estimate the load on the spring by measuring its displacement with the capacitance gauge. A known load was then applied by the loading device, and the corresponding load seen by the spring was measured. Using this technique, the frictional force exerted by the o-rings at an internal pressure of $220 \mathrm{bar}$ was approximately $200 \mathrm{~N}$.

During some creep tests, the gas pressure was accidentally dropped for a short period of time. This had the effect of temporarily increasing the creep rate by reducing the friction. Since the stress exponent of creep was known for this material (from air tests) it was possible to make a second estimate of the frictional force exerted by the o-rings by comparing the creep rate with and without gas pressure. Using this technique, a value of approximately $200 \mathrm{~N}$ was also deduced.

\section{Testing Procedures}

\section{Specimen Mounting}

The specimen gauge diameter was measured with a micrometer in at least five different locations to give an average cross sectional area which was then used to calculate the required test load. Thermocouples were then attached to the top and bottom shoulders, and the specimen was mounted in the load train. For all the tests in the thesis, only specimen was tested at a time (not two in series). The capacitive extensometer was only used on the first test, during which time it gave no useful results. The rig was then assembled and the LVDT for external measuring of the creep strain was placed in position and zeroed.

\section{Leak Testing}

Before going any further, the pressure chamber and gas infrastructure was leak tested by passing approximately 20 bar of helium through the system. Particular attention was paid to the seals on the load train. Leaks detection was carried out with a combination of the helium detector, Snoop soap solution, and pressure drop tests, which involve observing the change in pressure within the pressure chamber when both the inlet and outlet valves are closed. Once the 
rigs were confirmed leak free, the bell housing was put into place, and evacuation of the air commenced.

\section{Evacuation of the Bell Housing}

Evacuation of the bell housing involved lowering the pressure to approximately $0.5 \mathrm{~atm}$., and back filling with argon gas. This procedure was repeated up to five times until the oxygen content of the bell housing was around $0.3 \%$. To further reduce the oxygen content, a steady flow of argon at about $1.2 \mathrm{~atm}$. was fed through the bell housing. After a few hours, the oxygen content was normally reduced to $0 \%$ (within the resolution limits of the detector).

\section{Heating}

Once the oxygen content was sufficiently reduced, the furnace was turned on and allowed to heat up to around $500^{\circ} \mathrm{C}$. Thanks to the thermocoax heating system, temperature was reached relatively quickly (normally within a couple of hours). The final heating to $600^{\circ} \mathrm{C}$ was then carried out in small, well controlled steps, in which the four individual temperature controllers were independently adjusted.

\section{$\underline{\text { Alarms }}$}

Before pressurisation, the safety alarms had to be checked and set. Both forewarning and critical alarm levels were set, with only the critical alarm able to cause shut down of the experiment. The oxygen alarm levels of the bell housing were set at $3 \%$ and $4.5 \%$ respectively. In addition, hydrogen alarms connected to the bell housing exhaust, the garage, the gas trunking and the control room were set at 5 and $10 \%$.

\section{Pressurisation}

Hydrogen pressurisation was performed by increasing the pressure of argon in the high-pressure argon chamber. A mechanical device then allowed an slightly lower (-1.5bar) pressure of hydrogen to be introduced into the hydrogen pressure chamber. In this way the hydrogen was controlled by the safety gas, so that if the safety gas supply was cut off, so would be the hydrogen. All hydrogen detectors were carefully monitored during pressurisation, and the pressure was introduced slowly, over a period of several minutes. 


\section{Loading}

Once pressure and temperature were stable, the uniaxial creep load was applied. This was applied slowly, using the Coreci PID controllers, over several minutes to avoid accidental overloading. Once at the desired test load, the test start time was recorded.

\section{Data Logging}

A reference book for each reinforced concrete cell was used to store information about each test, including start time, temperature, pressure, material etc. In addition, periodic readings of the actual temperature, pressure, load and strain from the LVDT were recorded. However, the majority of data logging took place on a Siemens ${ }^{\mathrm{TM}}$ computer running a data acquisition programme written in the Labview ${ }^{\mathrm{TM}}$ programming language. This programme read the time, temperature, pressure and extensometry signals every few minutes, and wrote the data to file at specified intervals (normally every $1 / 2-1$ hour). 


\subsection{Internally Pressurised Tube Testing}

A rig already existed at the JRC for the testing of internally pressurised tubular components under hydrogen or argon environments, and had been previously used to study the creep crack growth behaviour of notched tubes under hydrogen [Looney'94]. However, there was no extensometry available which was capable of measuring hoop strain evolution with time under hydrogen environments, and therefore some modifications for the current work were necessary. A schematic of the rig after modification is shown in figure 4.23. The following section describes the basic rig structure with particular emphasis on the design of the new extensometry. As far as the author is aware, no results have yet been published of hydrogen pressurised tubes where the hoop strain has been continuously measured.

\section{Active Gas Containment and Control in the Tubular Testing Rig}

\section{$\underline{\text { Specimen Pressurisation }}$}

Hydrogen gas pressures of over 270bar are available from the compressor, although control becomes less certain as this pressure is approached. This gas is fed directly into the top of the specimen via a Tescom PID regulator. A small flow from the bottom of the specimen is maintained to allow better control of the pressure and to ensure continuity of the environment.

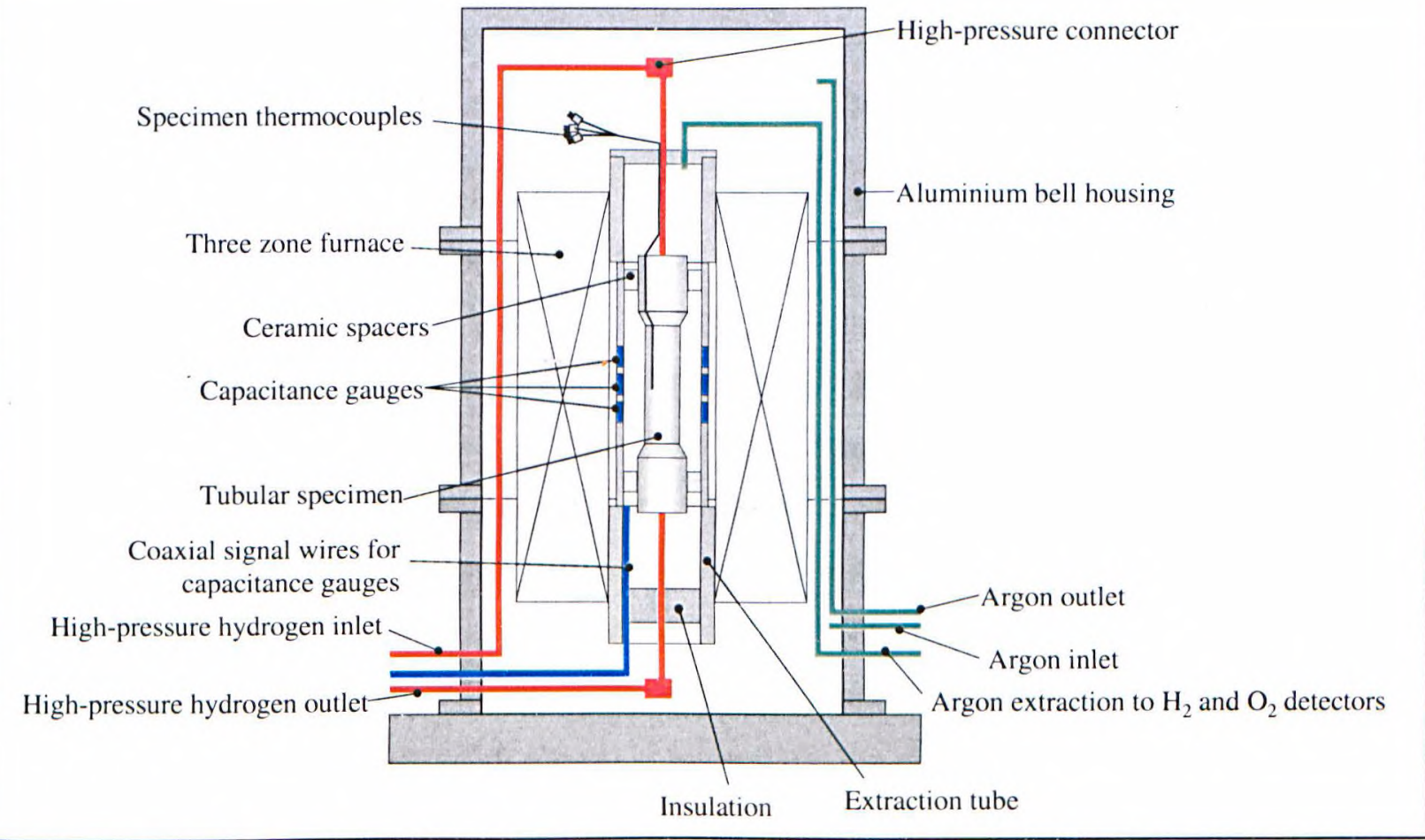

Figure 4.23: Schematic of the internally pressurised tube testing rig 
When the specimen was pressurised with argon gas, the following safety features were not required.

\section{Blanket gas}

Whilst the pressurisation of the specimen appears relatively straightforward, there are a couple of additional blanket gas features without which the tests would be too dangerous to run. In the event of a tube failing suddenly the rapid outflow of high pressure hydrogen must be contained to prevent a devastating explosion. To cover for this eventuality, the whole testing rig including the furnace is surrounded by a large aluminium bell housing filled with approximately 1.5 bar argon. The oxygen content of this bell housing is constantly monitored to ensure that a chemical explosion is impossible.

Previous experience at the JRC showed that the rate of hydrogen diffusion through the specimen wall during a test was significant, and could cause a build up of hydrogen within the bell housing if not removed. To overcome this, an extraction system was devised whereby the specimen is surrounded by a long pipe through which a constant flow of argon is flushed to the outside world. The hydrogen content of this flow is monitored, and allows a good indication of when a specimen is about to burst since the hydrogen content increases as the tube deforms, cracks and becomes thinner.

\section{Gas Supply Infrastructure}

The gas supply infrastructure is much the same as that for the HCR rigs, described in section 4.3. The main difference is in the extraction tube, which draws away any hydrogen that has diffused through the specimen wall, as mentioned above.

\section{Heating of the Tubular Specimen}

Heating of the specimen was performed using a three zone 3 kilowatt Mike Lockwood furnace positioned around the gas extraction pipe. Three ' $S$ ' type thermocouples measured the furnace temperature in each of the zones, and provided the input for independent Eurotherm PID controllers. The specimen temperature was measured with three externally mounted sheathed ' $\mathrm{N}$ ' type thermocouples positioned at the top, middle and bottom of the gauge length. It was found that the best temperature control was achieved when a rings of insulating material were inserted into the extraction pipe above and beneath the specimen to reduce heat loss by convection. Additional insulation on top of the furnace further improved the temperature 
control. Due to the high combined mass of the extraction tube and the specimen, the temperature response of the specimen to changes in the controllers' set points was relatively slow. As a result, the temperatures tended to drift up and down by as much as $1{ }^{\circ} \mathrm{C}$ in slow cycles of several hours. This is still within the recommended practice set out by the HTMTC [HTMTC'89]. The temperature differences between the three specimen thermocouples were normally less than $1^{0} \mathrm{C}$.

\section{Hoop Strain Extensometry}

\section{The Design Problem}

As with the HCR rigs, the design of the extensometry posed several problems. At the JRC, hoop strain is often measured by placing two opposed contacting rods perpendicular to the middle of the specimen gauge length. These rods then lead out through holes in the furnace where any movement can be measured by LVDTs. However, both the extraction pipe and the limiting size of the bell housing made this option rather difficult in the hydrogen rig. In addition, tubes tend to bulge during expansion, so measuring the hoop strain in only two positions may not accurately capture the strain at the maximum point of bulging. Some sort of multiple gauge, in-situ extensometry was required. Once again, a capacitance based system was decided upon, with the design and fabrication being jointly carried out with SJB Engineering, Bristol.

\section{The 12-Gauge Capacitance Transducer}

Unlike the PREDICH extensometry, these gauges work by measuring the change in distance between the two plates of the capacitor, rather than the change in area of overlap. The specimen forms one plate of the capacitor, and the gauges form the other. When an alternating voltage is applied across the plates, the capacitive reactance becomes linear with the gap between the plates. Twelve independent gauges arranged in three horizontal rings of four were used as illustrated in figure 4.24. The advantage of such a set up is that a profile of the tube can be drawn during the test, so that any bulging can be detected, and the position of maximum hoop strain can be determined. However, using each gauge individually is subject to potential error due to concentricity problems, so for accurate strain measurements the average of each set of four gauges is taken.

The gauges themselves, each measuring approximately $1.5 \times 1 \mathrm{~cm}$ are mounted into a three section stainless steel pipe, which forms the extraction pipe mentioned earlier. However, for the 
Numbers represent the individual gauges

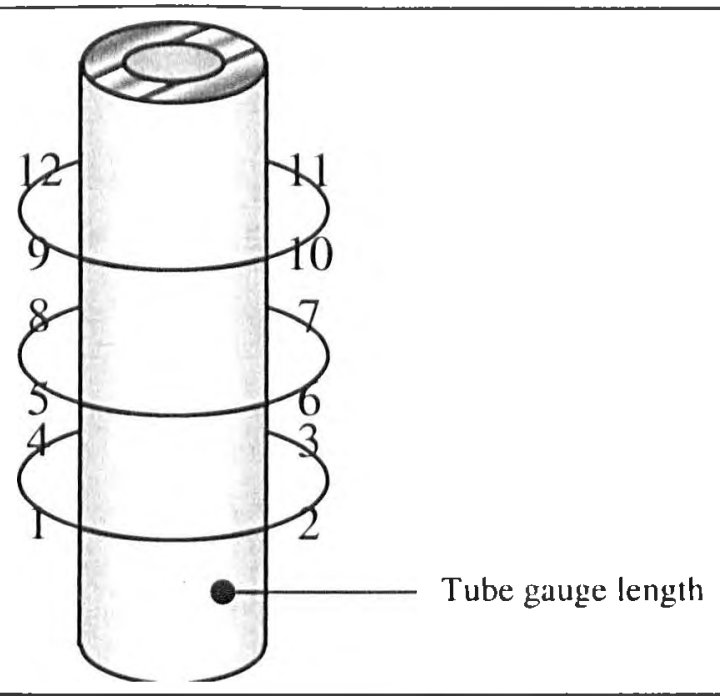

Figure 4.24: Positions of the 12 independent capacitance ganges around the tubular specimen gauge length

gauges to operate properly they must be isolated from this pipe. Isolation was originally obtained by using a fine ceramic paste which sets when heated. Later modifications resulted in the stainless steel housing being thermally sprayed with aluminium oxide such that the paste was no longer required. The use of the oxide coating made assembly much easier and quicker, as well as providing a more reliable isolating medium. In addition, the three sections of the extraction pipe are electrically isolated from each other. A schematic illustration of the gauge construction is shown in figure 4.25. It was necessary to ensure that the gap between the specimen and the gauges left enough room for straining, whilst remaining small enough to reduce electrical noise caused by having too large a gap. Taking a maximum expected specimen strain as $50 \%$, the inner diameter of the mounting for the gauges was machined to $66 \mathrm{~mm}$. This proved sufficient, except when a burst type failure occurred, in which case the specimen had a tendency to become stuck in the extraction pipe.

\section{Calibration of the Gauges}

For calibration and data logging purposes, the capacitive reactance was converted electrically to a voltage output, so that calibration plots could be made of voltage against tube diameter. Room temperature calibration was performed by inserting aluminium rods of various diameters ranging from $45-65 \mathrm{~mm}$ into the gauge. The rods were held concentric by plastic rings placed around the top and bottom of the gauge. Each individual gauge was calibrated separately, although it was found that the accuracy of the calibration due to concentricity problems ranged from about $\pm 0.5-5 \%$ as the diameter of the calibration rod changed from $45-65 \mathrm{~mm}$ respectively. Whilst these calibrations could be used to give an indication of the tube profile 
during a test, they were not accurate enough to plot strain vs. time curves with any confidence. To overcome this, the average value of each lateral set of four gauges was measured to give an average hoop strain at the top middle and bottom of the gauge length. When this method was

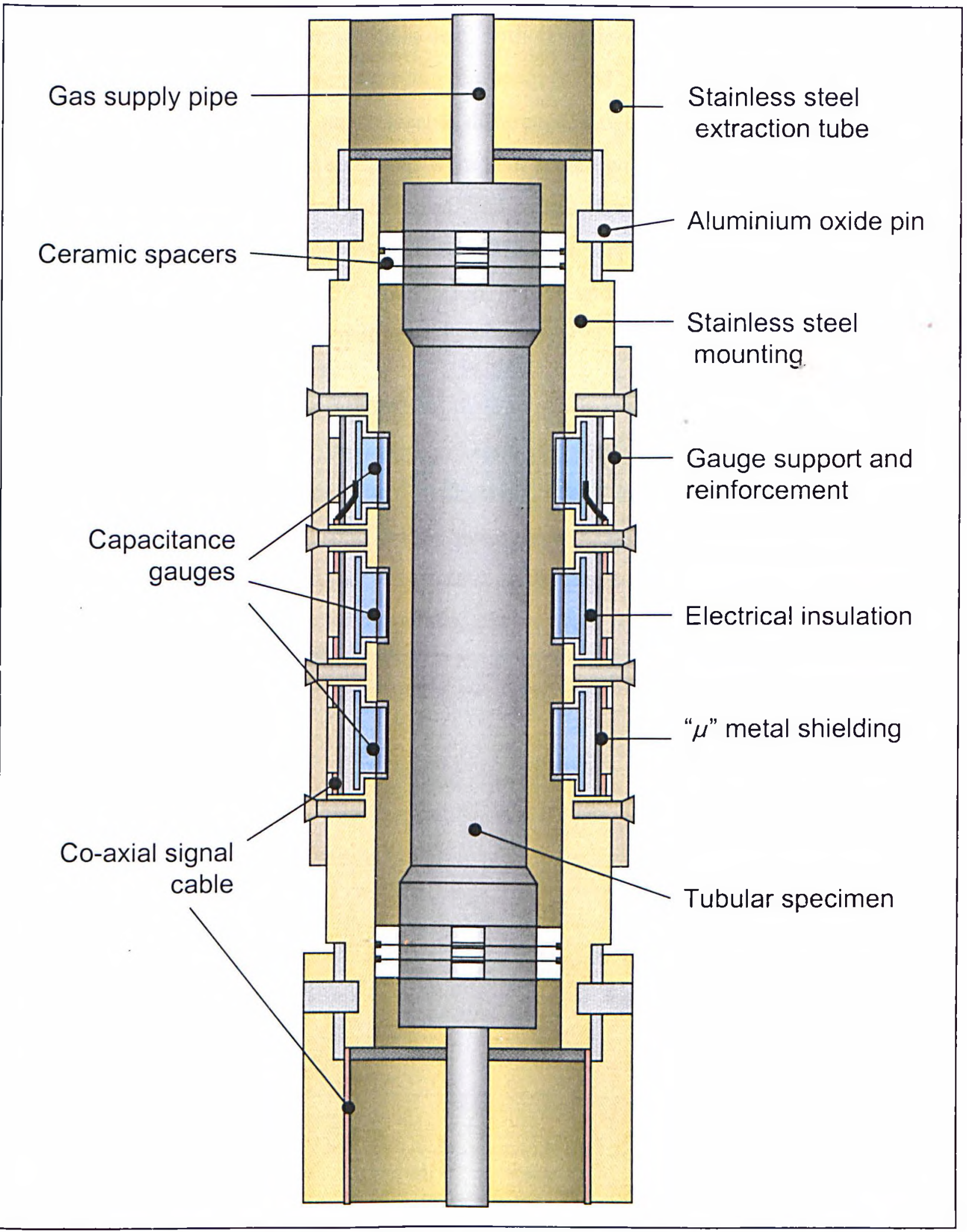

Figure 4.25: Schematic of the 12 gauge capacitance transducer for measuring in-situ the hoop strain evolution of internally pressurised tubes at elevated temperature 
used, the error due to concentricity diminished by a factor of about 10 , such that the worst error incurred by the $65 \mathrm{~mm}$ rod was only $\pm 0.5 \%$.

To ensure that the calibration remained accurate at high temperature, a second set of calibration rods were made, this time from stainless steel with ceramic spacers. These showed that there was negligible difference between the room temperature and high temperature behaviour of the gauges. In addition, there was no noticeable difference between using aluminium or stainless steel rods. Some of the stainless steel rods were later coated with a thin film of plastic to test the effect of surface condition on the calibration. The plastic coating caused a small zero offset on the calibration curve, but did not significantly effect the slope. A typical calibration obtained with the stainless steel rods for the average top, middle and bottom gauges is shown in figure 4.26. In general the device was sensitive to displacements of $10 \mu \mathrm{m}$.

\section{Initial Trials With the 12-Gauge Transducer}

Early trials with the transducer showed unexpected properties during heating of the specimen. As the temperature rose the signals became very noisy until they eventually showed a baseline signal, often characteristic of a short circuit between the gauges and the specimen. After some investigation, it was discovered that the ceramic paste used to isolate the three sections of the extraction pipe had semi-conductor properties, and therefore became conducting during heating,

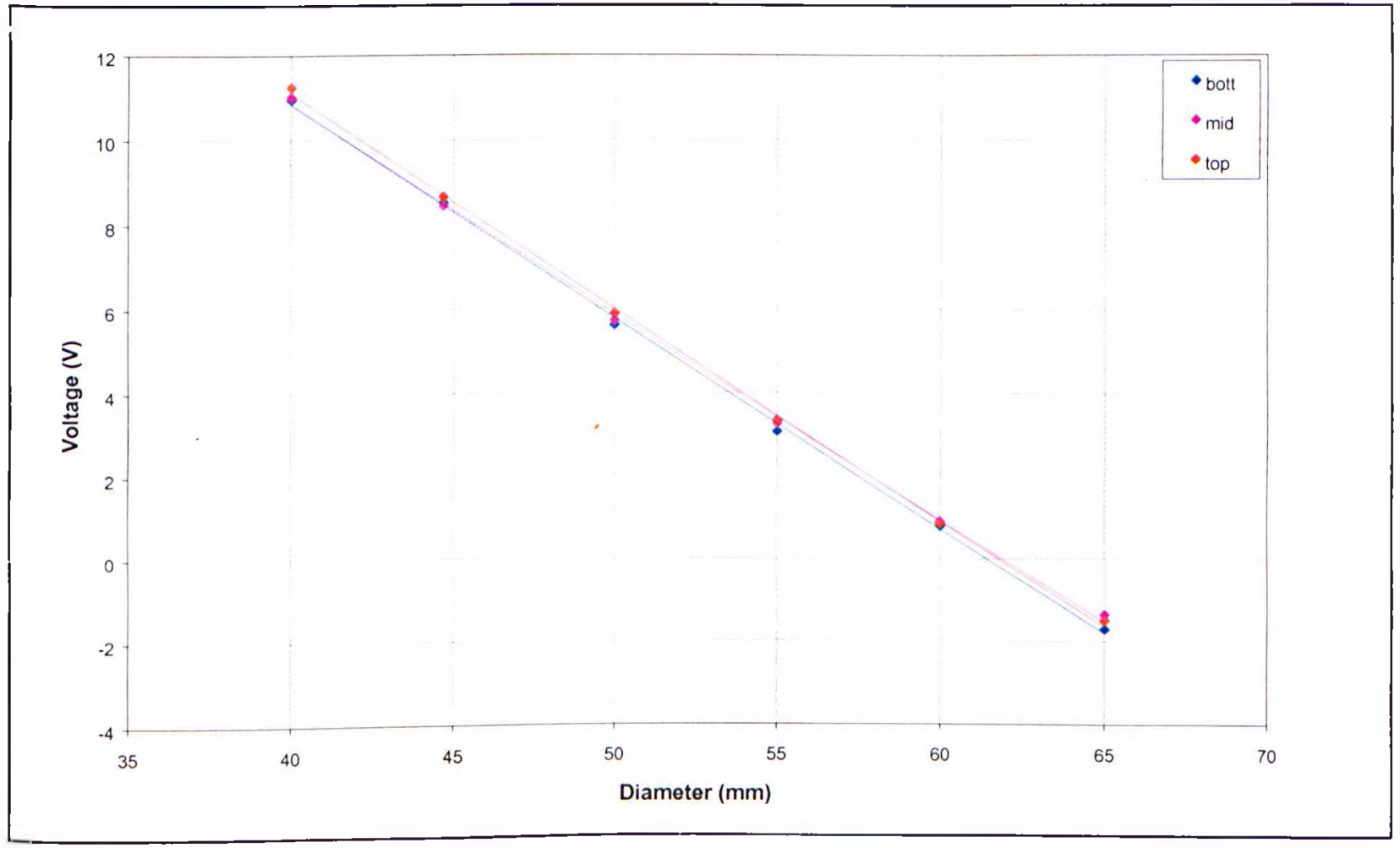

Figure 4.26: Calibration curve for the average of the top, middle and bottom set of four gauges in the tubular specimen testing rig 
causing the shorting with the specimen. The problem was overcome by changing the type of ceramic paste used. A similar effect of noise and shorting occurred sometime afterwards although, this time, it was caused by cracking of the ceramic casing around the metallic pins which held the extraction pipe sections together. The problem was overcome by replacing the ceramic coated metal pins with larger, entirely ceramic pins. The joining techniques employed before and after these modifications are shown in figure 4.27. All of these problems were finally overcome by using the aluminium oxide coating.

Another form of shorting still seemed to be occurring during heating, although it generally sorted itself out after some time at temperature. It is well known that the insulation used for the three zone furnace is extremely hydroscopic, and it was postulated that during initial heating the water was being driven out of the furnace where it could subsequently soak into the ceramic paste (or even the aluminium oxide coating) which isolated the gauges from the middle section of the extraction tube. In addition, the isolation material used in the coaxial cables becomes slightly conductive when wet. After holding at temperature for some time, the system dried out again and the problem disappeared. To minimise this effect, several containers of silica gel were introduced into the argon blanket gas, and the furnace was held at around $60^{\circ} \mathrm{C}$ in-between tests to reduce the amount of water re-adsorbed.

Almost the same symptoms again were the result of yet another unforeseen difficulty. Noise, sometimes followed by shorting occurred in some of the tests after several hundred hours of testing. The cause of this were spallations on the specimen which in severe cases could bridge

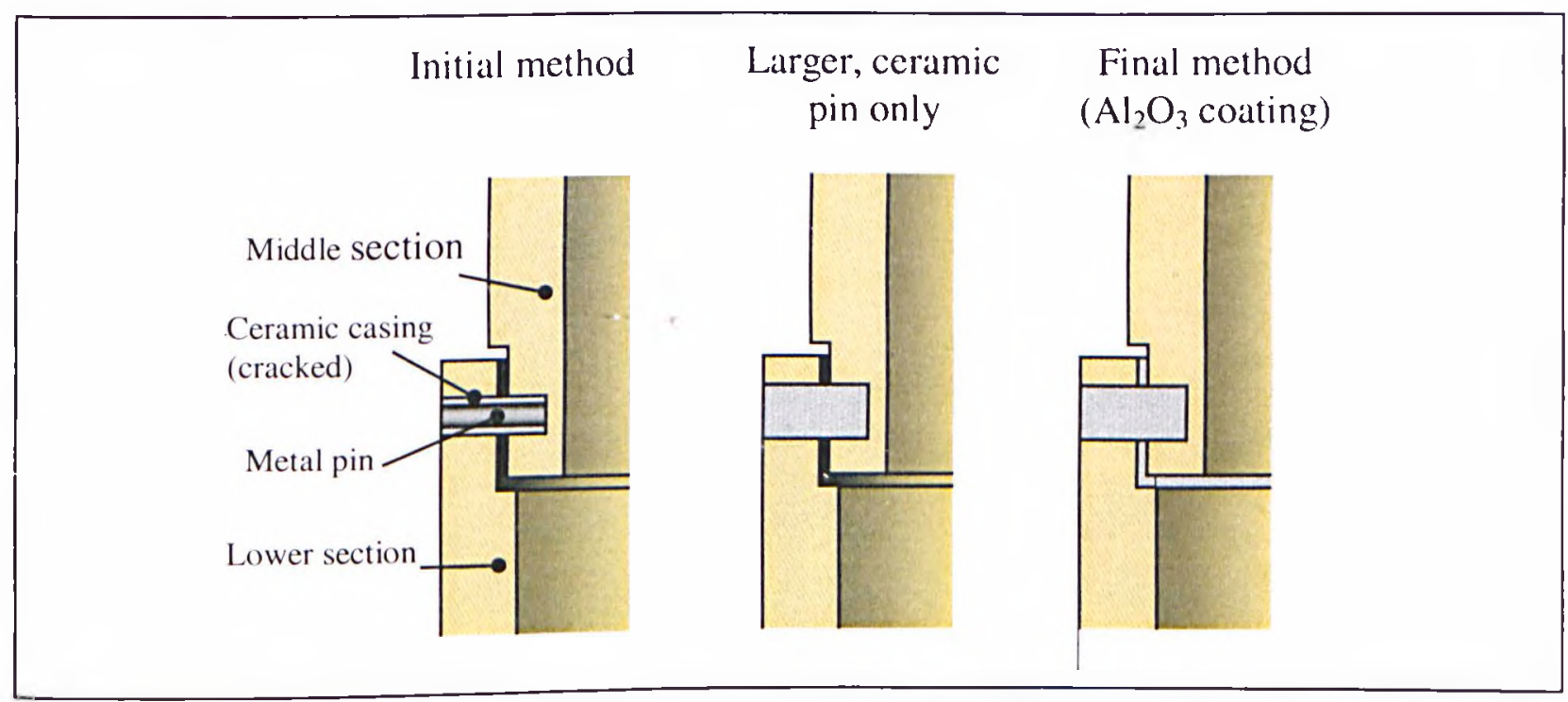

Figure 4.27: Joining techniques for connecting the middle and lower sections of the extraction tube and gange mounting. The same techniques are mirrored for connecting the top and middle sections 
the gap between the specimen and the gauges (Figure 4.28). During hydrogen exposure, a matt silvery grey finish appears on the tube, both on the inside and the outside surfaces. A similar phenomena has been noticed in the literature [Bramle.'32], [Looney'94], and on other specimens exposed in the work of this thesis. On the outside of the tube, this finish may actually peel of in thin strips, especially during bulging, in which case it can cause shorting. Further analysis of this surface layer can be found in Appendix A, where it is argued that the layer is a form of reduced oxide. To minimise the effect of the spallations, it was therefore decided to reduce the amount of oxide on the specimen surface. This was achieved by ensuring that the specimen was thoroughly cleaned before testing, and heating was delayed until the argon blanket gas was fully deoxidised. Whilst this seems to have reduced the problem somewhat, it did not always completely solve it, and as such it was necessary sometimes to stop an experiment and clean the specimen before restarting.

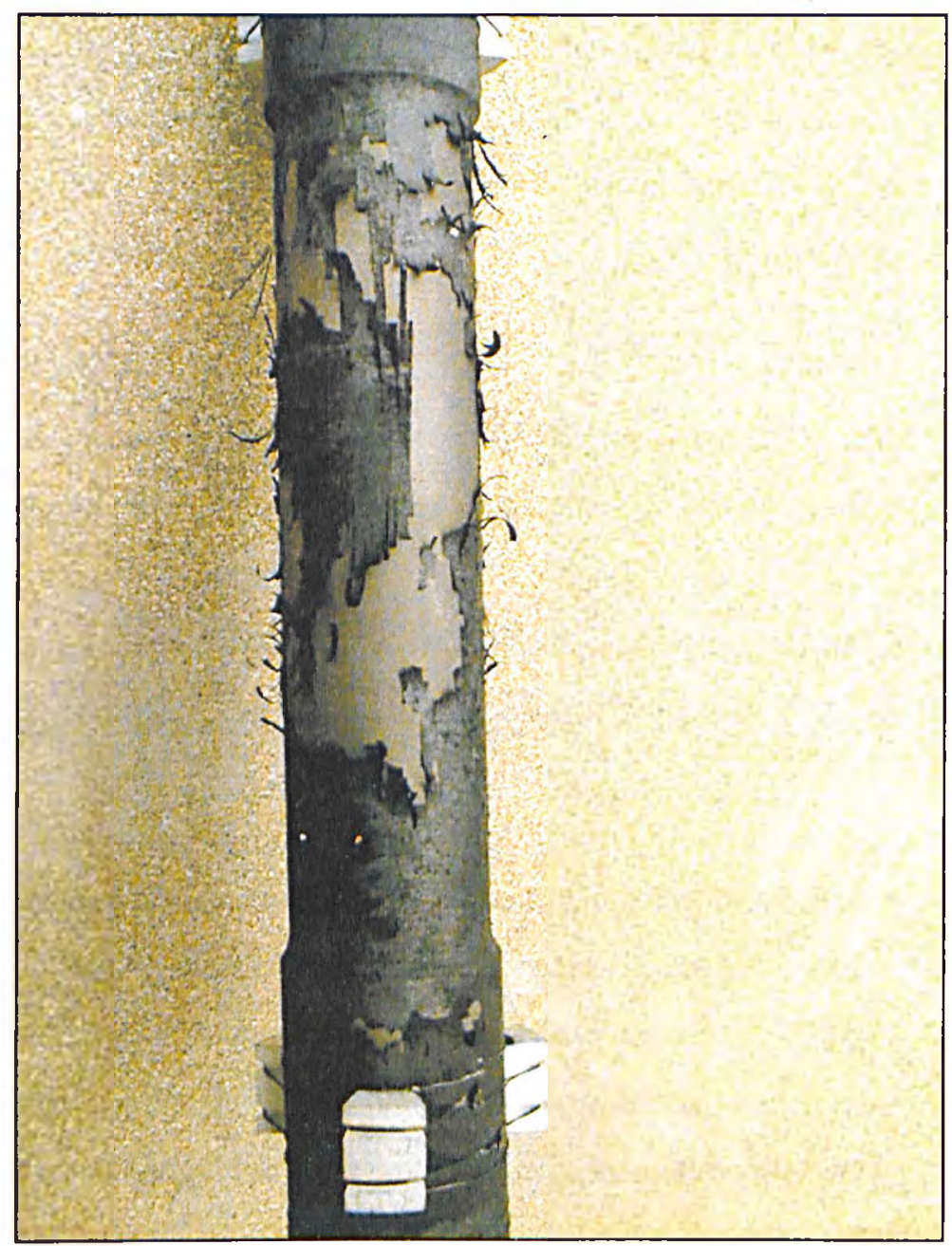

Figure 4.28: Surface spallation on the external side of a tube tested under 19.5bar hydrogen at $600^{\prime \prime} \mathrm{C}$ which caused an electrical short circuit between the specimen and the capacitance gauges 


\section{Testing Procedures}

\section{Specimen Mounting}

Before mounting the specimen, it was first necessary to position the ceramic spacers around the shoulders as shown in figure 4.25. Next the thermocouples were spot welded to the specimen shoulders, and one was positioned so that it was in contact with the centre of the gauge length. Finally, insulation material was positioned around the gas supply pipes to help keep good temperature control during the test. Once instrumented, the specimen was then mounted in the rig by two HIP high-pressure connectors at the top and the bottom. The gas outlet from the extraction tube was then fitted and the top of the furnace was covered with insulation material.

\section{Leak Testing}

Before going any further, the tube and gas infrastructure was leak tested by passing approximately 20 bar of helium through the system. Leaks (when present), were detected with a combination of the helium detector, Snoop soap solution, and pressure drop tests, which involved observing the change in pressure within the specimen when both the inlet and outlet valves were closed. Once all leaks had been fixed, the bell housing was put into place, and evacuation of the air commenced.

\section{Evacuation of Bell Housing}

Evacuation of the bell housing involved lowering the pressure to approximately $0.5 \mathrm{~atm}$., and back filling with argon gas. This procedure was repeated up to five times until the oxygen content of the bell housing was around $0.3 \%$. To further reduce the oxygen content, a steady flow of argon at about $1.2 \mathrm{~atm}$. was fed through the bell housing. After a few hours, the oxygen content was normally reduced to traces (within the resolution limits of the detector).

\section{Heating}

Once the oxygen content was sufficiently reduced, the furnace was turned on and allowed to heat up to around $500^{\circ} \mathrm{C}$. As a consequence of the large volume of metal within the furnace, this heating took several hours to complete. The final heating to $600^{\circ} \mathrm{C}$ then took place in small, well controlled steps, in which the individual temperature controllers were independently adjusted. To ensure an even temperature gradient, the specimen was normally left overnight to settle. 


\section{$\underline{\text { Alarms }}$}

Before pressurisation, the safety alarms were checked and set. Both fore and critical alarm levels were set, with the fore alarm being a warning only, and the critical alarm causing shut down of the experiment. The oxygen alarm levels of the bell housing were set at about $3 \%$ and $4.5 \%$ respectively. In addition, hydrogen alarms connected to the extraction tube, the bell housing, the garage and the control room were set at 5 and $10 \%$.

In addition to the gas detectors, alarms were also set for the hydrogen gas pressure. Both a low set point alarm, and a high set point alarm were set. Initially the low-pressure alarm was turned off to avoid shutting down of the furnace before any pressure was introduced. However, after pressurisation, this alarm was set at about 5-10 bars below the test pressure. In this way it should be normally sensitive enough to measure the drop in pressure just prior to failure, without being sensitive to small fluctuations in the test pressure. A high set point alarm was also set to prevent accidental over-pressurisation caused by compressor or controller failure.

\section{Pressurisation}

Finally, the hydrogen gas was slowly introduced. All hydrogen detectors were carefully monitored during pressurisation, and the pressure was introduced slowly, over a period of several minutes. Once at pressure the low-pressure alarm was set, and the test start time was recorded.

\section{Data Logging}

A reference book for each reinforced concrete cell was used to store information about each test, including start time, temperature, pressure, material etc. In addition, periodic readings of the actual temperature, pressure, and voltage signals from the capacitance transducer were recorded. However, the majority of data logging took place on an Olivetti ${ }^{\top M}$ computer running a data acquisition programme written in the Labview ${ }^{\top M}$ programming language. This programme read the time, temperature, pressure and extensometry signals every few minutes, and wrote the data to file at specified intervals (normally every $1 / 2-1$ hour). 


\subsection{Test Conditions}

The test conditions for all tests are given below. Note that all of the different types of test were carried out at $600^{\circ} \mathrm{C}$. This temperature was chosen as it allowed acceleration of both the creep rate and the hydrogen attack kinetics. In addition, it was the same temperature used in a recent study of hydrogen attack assisted creep crack growth of $2.25 \mathrm{Cr}-1 \mathrm{Mo}$ steel carried out at the JRC [Looney'94], which could allow for some comparison between the results.

\section{Uniaxial Creep in Air}

Four tests were performed, under the following stresses: $110,100,95,90 \mathrm{MPa}$

\section{Uniaxial Creep in Hydrogen}

All tests were carried out under a pressure of 220bar. 220bar was chosen at it was approximately the average pressure used in tube testing (see below). The following table summarises the loads and subsequent stresses used, bearing in mind the frictional force present on the load train. In addition, two of the tests were subject to a zero offset in their load due to drift of the load controller.

\begin{tabular}{|c|c|c|c|c|}
\hline $\begin{array}{c}\text { Applied Load } \\
{[\mathbf{N}]}\end{array}$ & $\begin{array}{c}\text { Load Offset* } \\
{[\mathbf{N}]}\end{array}$ & $\begin{array}{c}\text { Frictional Force } \\
{[\mathbf{N}]}\end{array}$ & $\begin{array}{c}\text { Specimen } \\
\text { Diameter } \\
{[\mathbf{m m}]}\end{array}$ & $\begin{array}{c}\text { Test Stress } \\
{[\mathbf{M P a}]}\end{array}$ \\
\hline 2143 & 100 & 200 & 4.980 & 104.9 \\
\hline 1948 & 100 & 200 & 4.980 & 94.9 \\
\hline 1900 & 0 & 200 & 4.995 & 86.8 \\
\hline 1850 & 0 & 200 & 5.005 & 83.9 \\
\hline
\end{tabular}

*For the first two tests a load offset had to be considered due to drift of the load controllers. This offset is approximately $100 \mathrm{~N}$, although there may be up to $\pm 30 \mathrm{~N}$ error in this.

In addition to these tests, an extra test was carried out in the rigs at a load of $1850 \mathrm{~N}$, but this time in an argon environment. When compared with control tests carried out in air, it allows some appraisal of the validity of taking $200 \mathrm{~N}$ as the frictional force (see Results section 5.3).

\section{Internally Pressurised Tubes}

Tubes were pressurised with hydrogen at 260,240,220 and 195bar hydrogen, and with argon at 260,240 and 220 bar for control experiments. 


\section{RESULTS AND DISCUSSION}

The following chapter describes the results determined through the experimental techniques discussed in chapter 4. Initially, the effect of hydrogen in the absence of applied stress on the test material is described, followed by a description of the material creep behaviour without hydrogen under both uniaxial and multiaxial stress. These initial sections provide baseline data against which the results of section 5.3, "Combined Creep-Hydrogen Attack Behaviour", can be compared. This section contains the only hoop strain creep curves determined from hydrogen pressurised tubes known to the author, rare uniaxial creep data in a hydrogen environment, as well as a detailed description of the damage mechanisms occurring. Some initial discussion is also presented in this chapter, regarding especially the correlation of the uniaxial and multiaxial test results. 


\subsection{Material Behaviour in Hydrogen Without Stress}

The majority of the work in this thesis is based around studying the effect of combined creep stress and hydrogen attack conditions in both uniaxial specimens and internally pressurised tubes. However, in order to see what effect creep has on hydrogen attack, and vice versa, it is first necessary to analyse the hydrogen attack phenomena in the test material, in the absence of stress. This section will describe the results of autoclave exposures, whilst the following sections will concentrate on the effect of creep stresses, both with and without the presence of hydrogen.

\section{Hydrogen Attack Cavitation in the Absence of Applied Stress}

Autoclave exposures were performed under the same conditions as the hydrogen-pressurised tubes (section 5.3). In fact, the insides of the tubes were used as the autoclaves, giving exposures ranging in time from 123 to 715 hours, with pressures from 260 to 195bar respectively at $600^{\circ} \mathrm{C}$. These pressures at such a temperature are well within the 'unsafe' region of the Nelson curves [API'97], although the Nelson curves do not consider the effect of time of exposure. The majority of exposures were performed on specimens measuring approximately $1 \mathrm{~cm}^{3}$.

After exposure, the specimens were sectioned, polished, etched with $5 \% \mathrm{Nital}$ and examined with both the optical microscope and the SEM. Within the conditions studied, no hydrogen attack cavitation was detected in the tempered British Steel (BS) material.

\section{Observation Limits / Artefacts}

Cavities of less than about $0.25 \mu \mathrm{m}$ diameter could not be positively identified in the work of this thesis. It was difficult to distinguish with certainty between carbide "shadows", inclusions and cavities at this size scale. For example, figure 5.1 shows manganese sulphide particles in two steels, one exposed for 715 hours in 195bar hydrogen, the other for 573hours in 220bar argon (both at $600^{\circ} \mathrm{C}$ ). These inclusions had been sectioned perpendicular to their length, and were identified as MnS using EDS spot analysis. They should not be mistaken for cavities.

\section{The Surface Decarburised Zone}

Despite the lack of cavitation, another form of hydrogen induced damage, surface decarburisation, was clearly seen in the autoclave-exposed specimens. Surface decarburisation 


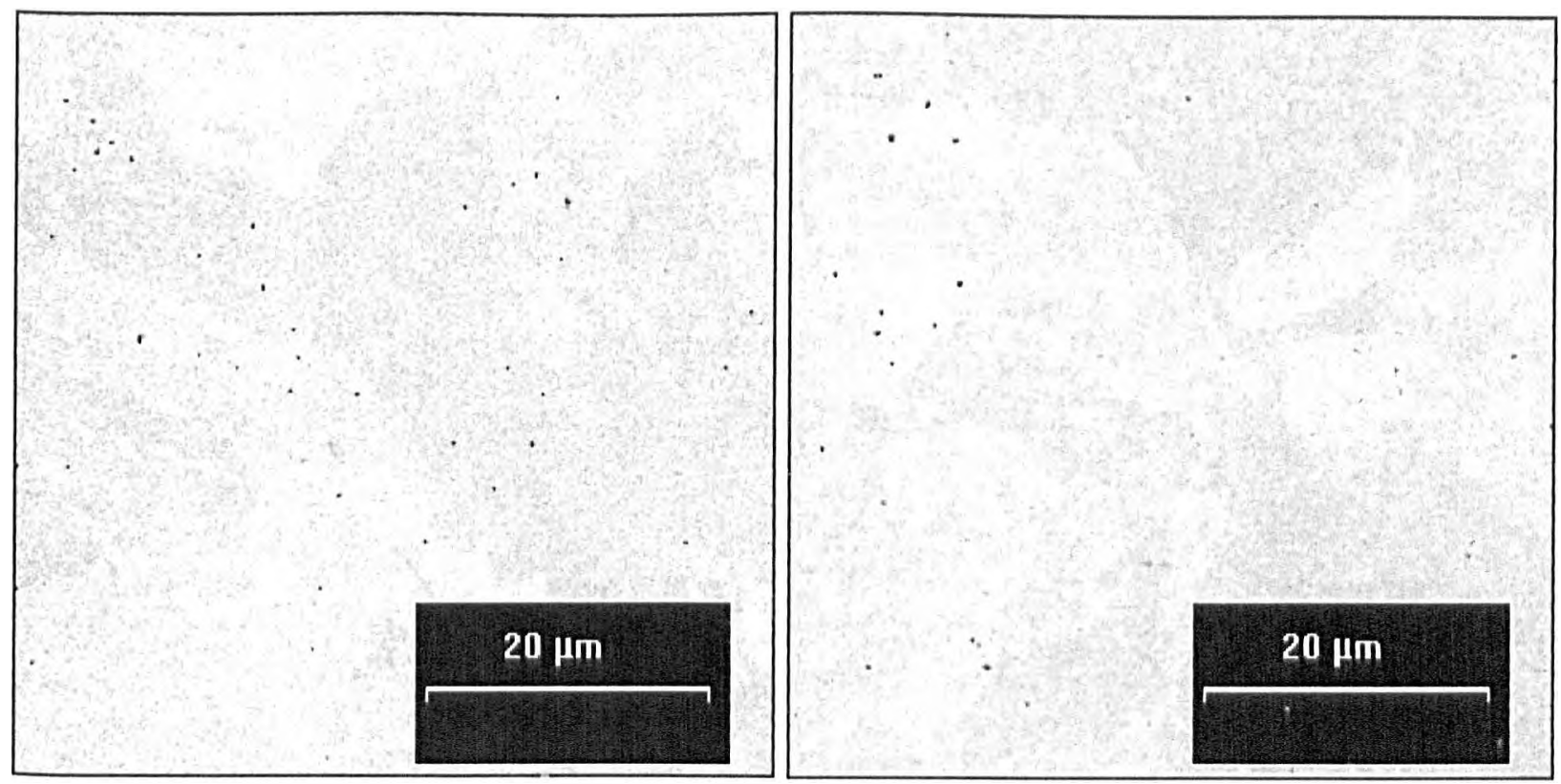

Figure 5.1: Manganese sulphide particles in material exposed to 220bars argon for 573hours (left), and exposed to 19.5bars hydrogen for 715 hours (right) at $600^{\circ} \mathrm{C}$. Note the absence of cavitation in the hydrogen exposed material and the potential to mistake MnS as small cavities

is often ignored in the hydrogen attack literature, its small size relative to the industrial pressure vessel wall thickness making it negligible in terms of its effect on creep strength. It is however a good place to study the hydrogen attack mechanism, after all, the decarburised surface is proof of the most fundamental phenomenon in hydrogen attack: the methane reaction. An exploratory study has been carried out to glean details of the hydrogen attack mechanism by exploiting the decarburised zone.

Observations were best made by sectioning, polishing and etching in 5\% Nital solution. With the naked eye, decarburisation can sometimes be seen as a thin shiny layer on the surface of the material, and is identified as a lighter coloured region with the optical microscope. The SEM shows very clearly the decarburisation, since individual carbides can be easily seen. Figure 5.2 compares the surface decarburised zone and the bulk of the specimen exposed for $715 \mathrm{hrs}$ under 195bar pressure. The number of carbides is drastically reduced near the surface. The depth of decarburisation is difficult to estimate, since there is in fact a gradient of carbon content from the surface inward [Masaok.' 82 ]. However, it was possible to determine for the temperature and pressures studied, that the depth of decarburisation depended mainly on time rather than hydrogen pressure.

Not all of the carbides near the surface were dissolved. Those which remained were examined closer to understand what it was which made them resistant to dissolution, and therefore 

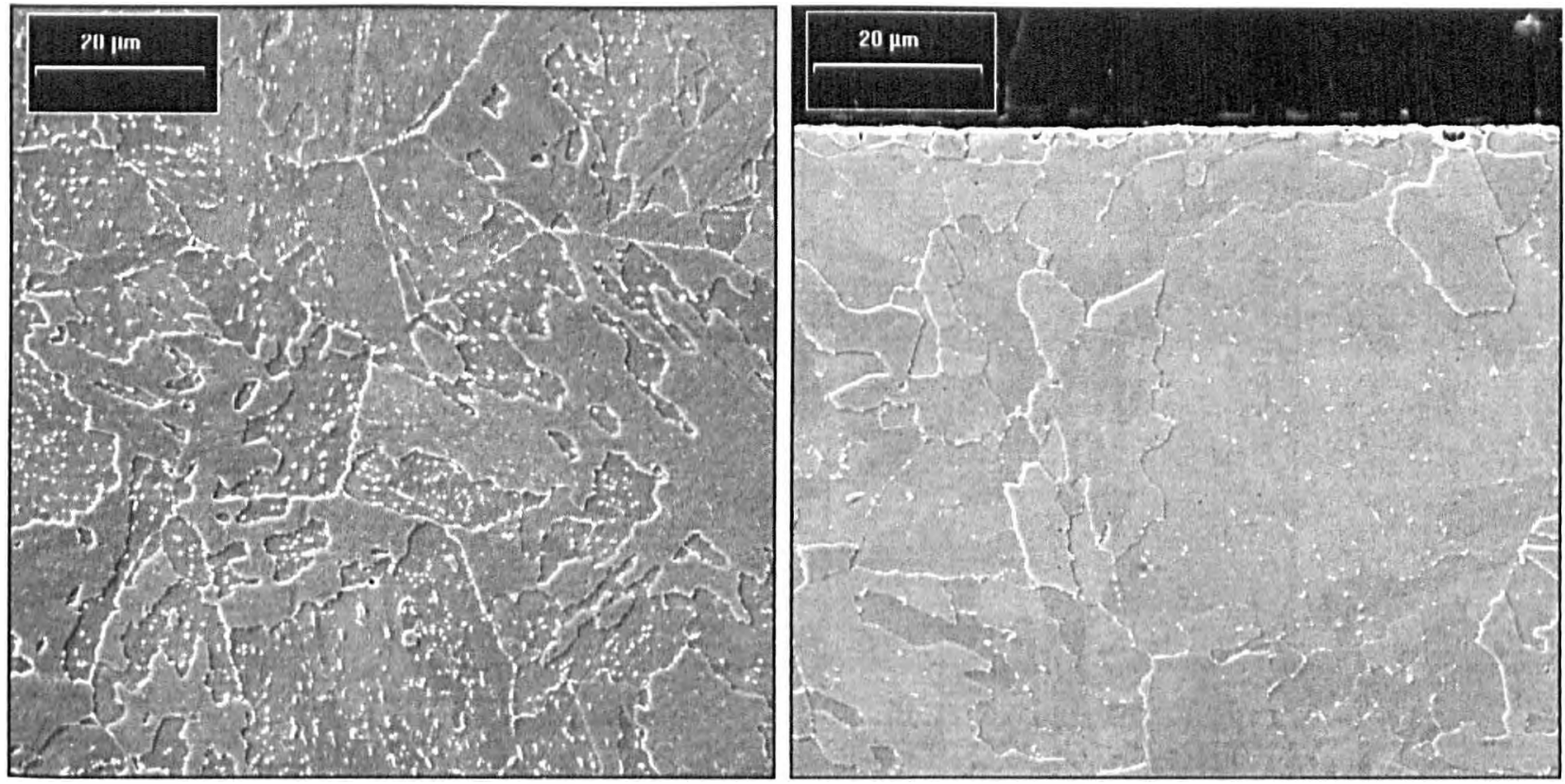

Figure 5.2: Comparison of the bulk (left) and surface decarburised zone right) of a specimen exposed to 19.5har hydrogen for 71.5hours. (etched in .5\% Nital and examined with the SEM)

resistant to hydrogen attack. Carbide extraction replicas were taken from the bulk and from the decarburised material at a depth of $4 \mu \mathrm{m}$. The depth was ascertained by careful micrometer readings and accurate weight measurements before and after polishing. Figure 5.3 compares the carbide situation in the bulk and in the surface of the specimen exposed to 195bar hydrogen for 715hours. A large decrease in the number of carbides is clearly seen. Systematic EDS' analysis and selected diffraction patterns show that the carbides in the decarburised zone have the $\mathrm{M}_{6} \mathrm{C}$ structure. The bulk material contains $\mathrm{M}_{6} \mathrm{C}, \mathrm{M}_{7} \mathrm{C}_{3}$ and traces of $\mathrm{M}_{2} \mathrm{C}$.

It is concluded that the most resistant carbide to dissolution in the $\mathrm{BS}$ steel is the $\mathrm{M}_{6} \mathrm{C}$ type, since no sign of the others remain in the decarburised zone. This is not surprising, as $\mathrm{M}_{6} \mathrm{C}$ is also the most stable carbide under long term tempering or ageing operations [Baker'59]. It may be possible to depth profile the steel by polishing back the surface little by little to see the order in which the other carbides appear in the decarburised zone. This might allow a stability ranking to be made which would be expected to closely follow the carbide stability found during ageing or tempering (see for example [Baker'59],[Yu'89]). However, this was beyond the scope of the thesis.

I Recent work at the JRC has shown that for a given steel composition the carbide chemical composition (determined with EDS) is directly related to the crystal structure, and can be used as a quick method for determining the carbide types in the steel |Manola. 981. 
DECARBURISED
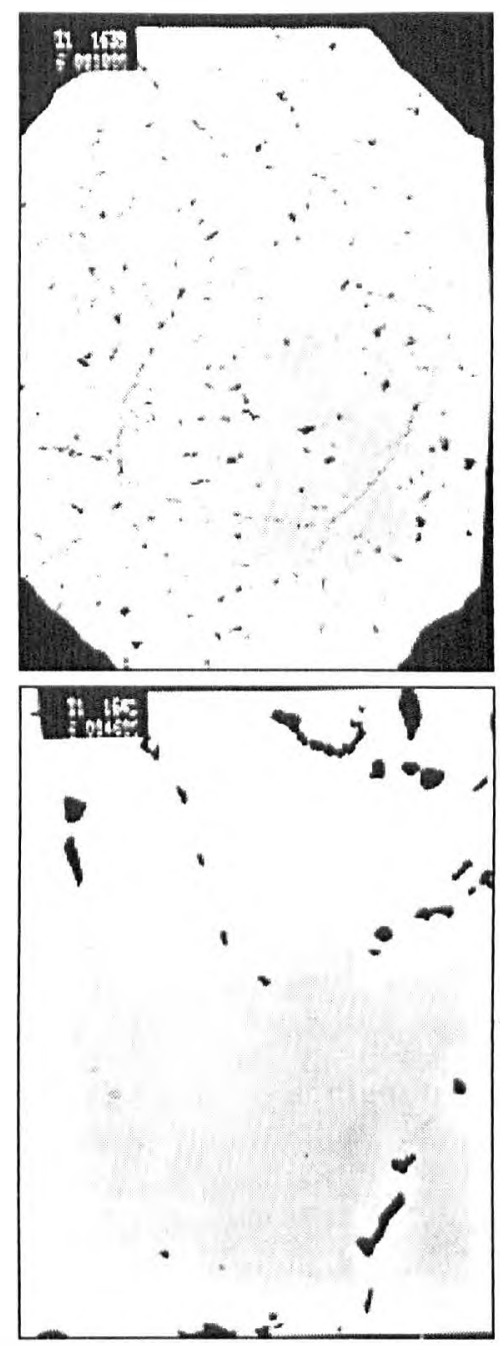
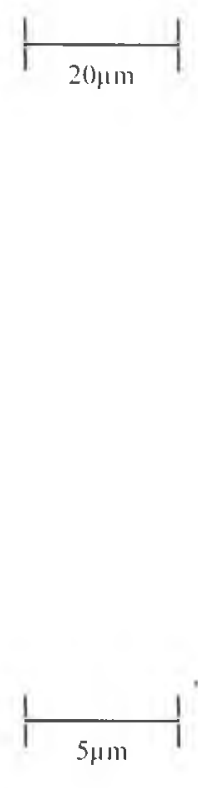

Figure 5.3: Comparison of extraction replicas taken from the decarburised surface and the bulk of a specimen exposed for 715 hours under 195 bar hydrogen. 


\subsection{Material Creep Behaviour in Air and Argon}

\section{Uniaxial Creep in Air}

Four uniaxial constant load creep tests have been carried out in an air environment at $600^{\circ} \mathrm{C}$. The stresses used were 110,100,95 and 90MPa. These data allow the basic material creep properties to be determined and later compared against creep in a hydrogen environment.

\section{Uniaxial Creep Curves}

Full creep curves were obtained for all tests as shown in figure 5.4. Throughout this thesis, strain will be written in terms of percentage engineering strain and time in units of hours. The creep curves show a minimal primary creep stage and very high ductility at this temperature. In general, the ductility decreases slightly with increasing test time, with the exception of the test at $100 \mathrm{MPa}$.

Figure 5.5 shows the material stress rupture plot and compares it with the ISO standard [ISO'81] for this material. The BS material has a somewhat lower rupture strength than the standard. This is probably due to the high tempering treatment which was applied to the material to reduce its' strength, resulting in a coarsening of carbides (section 4.1).

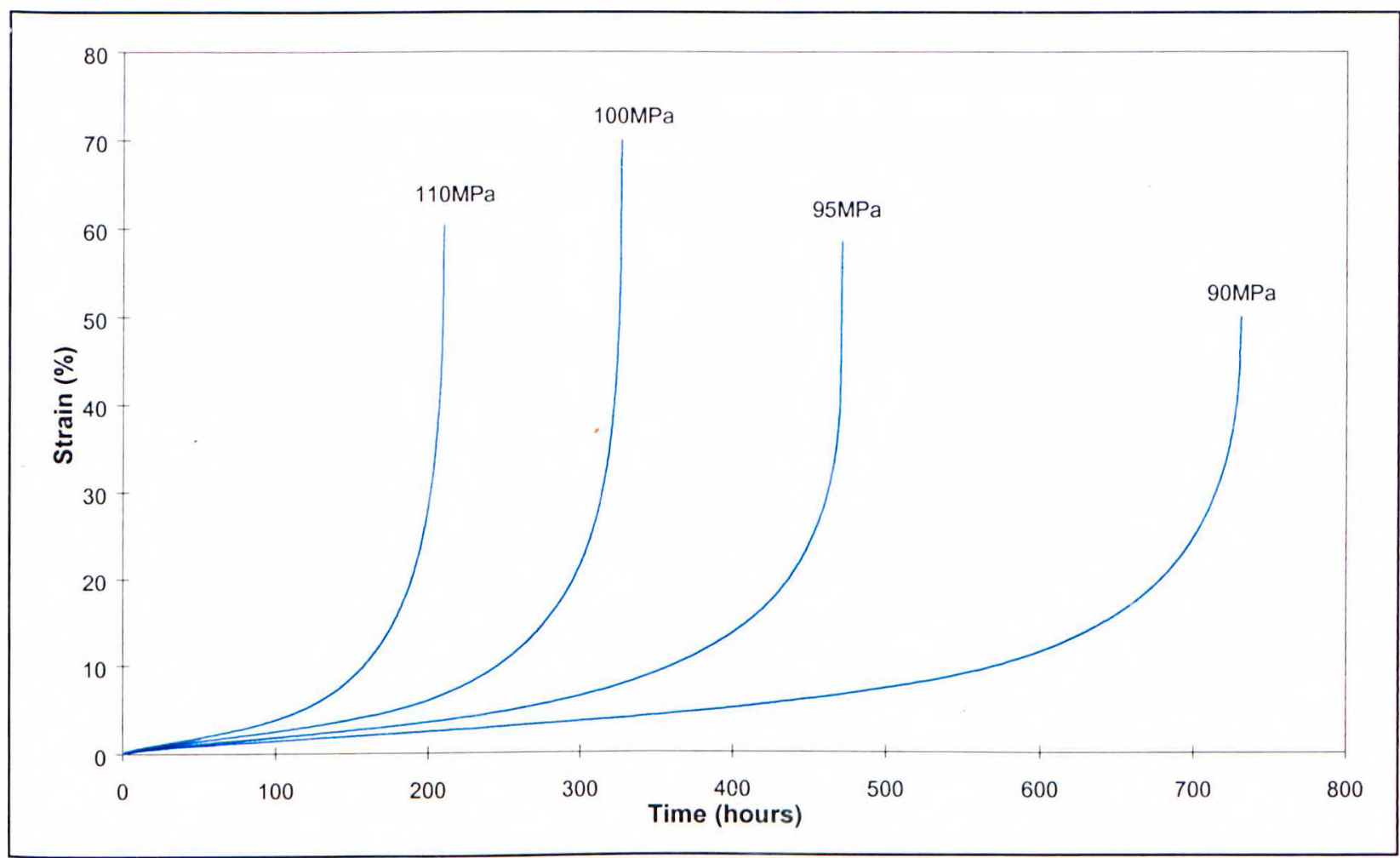

Figure 5.4: Uniaxial constant load creep curves of the tempered BS material in air at $600^{\circ} \mathrm{C}$ 


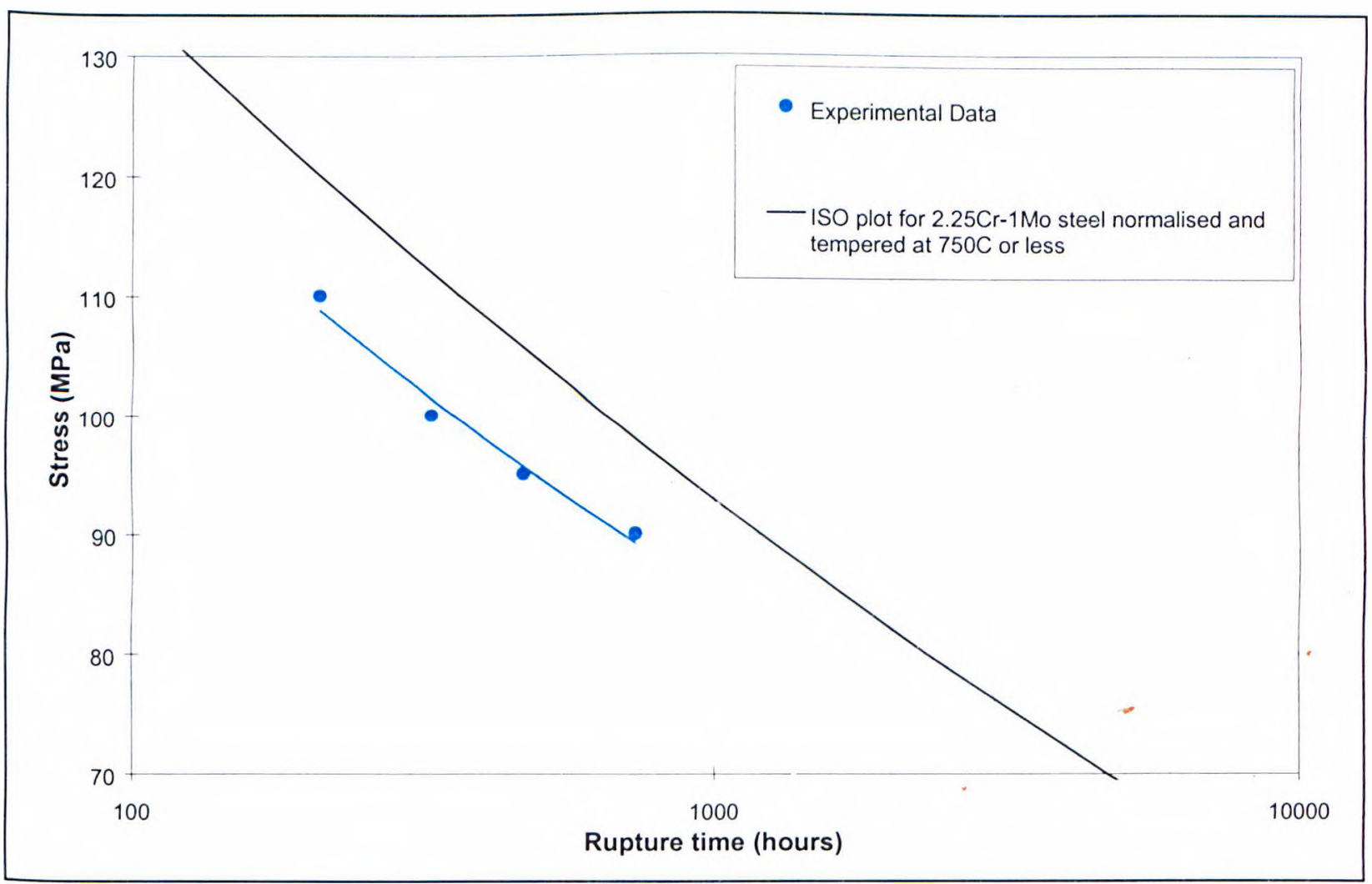

Figure 5.5: Stress-Rupture plot of the tempered BS material compared with the ISO rupture plot for 2.25Cr-1Mo steel normalised and tempered at $750^{\circ} \mathrm{C}$ or less.

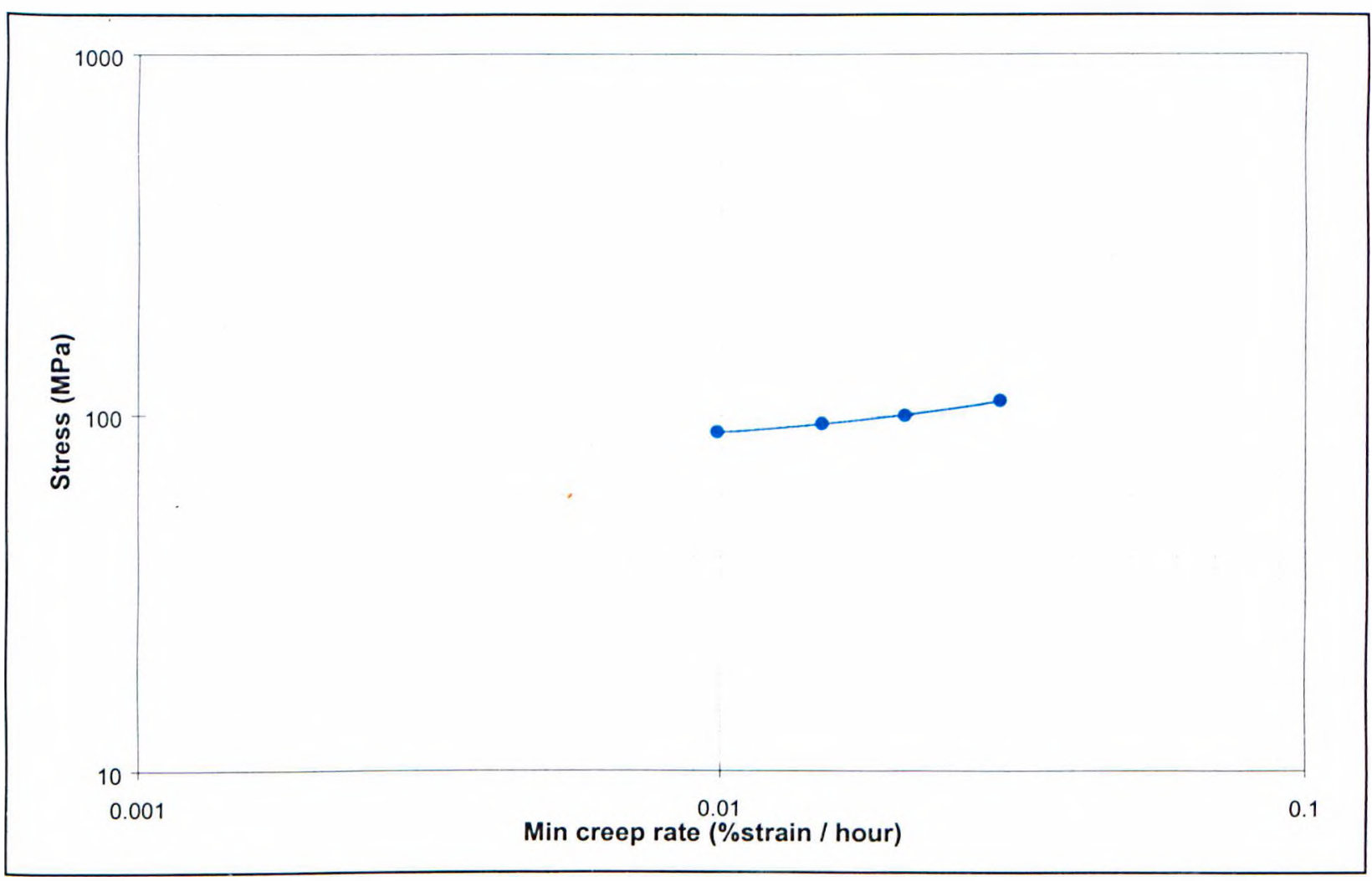

Figure 5.6: Log minimum creep rate vs log stress plot for the tempered BS material tested under constant load uniaxial tension 
The minimum creep rate was also obtained from the creep curves using the ASTM seven point method. This has been used to plot log rate vs log stress Norton plots (figure 5.6). Assuming that:

$$
\varepsilon_{\min }=K \sigma^{n}
$$

then from figure 5.6 it can be shown that $\mathrm{n}=5.46$ and $\mathrm{K}$ is $2.30 \times 10^{-13}$. These values (particularly $n$ ) are useful later when modelling and analysing the stress distribution with internally pressurised tubes (see section 6).

\section{Uniaxial Creep Ductility}

All specimens failed in a ductile manner, with large amounts of elongation and necking to give a small cross sectional area at failure (see for example figure 5.7). In fact, the reduction of cross sectional area (RA) for all the tests was greater than $90 \%$. However, unlike the strains to failure, there was no general trend to decrease the reduction of area with increasing test time.

\section{Metallographic Examination of Uniaxial Specimens:}

Sections of the specimens taken in the longitudinal direction were examined on the optical microscope to get more information on the failure mechanisms. Micrographs from the longest and the shortest term tests are shown in figure 5.8. It would appear that there is little difference in the micrographs, both showing high levels of grain deformation and little cavitation. Some

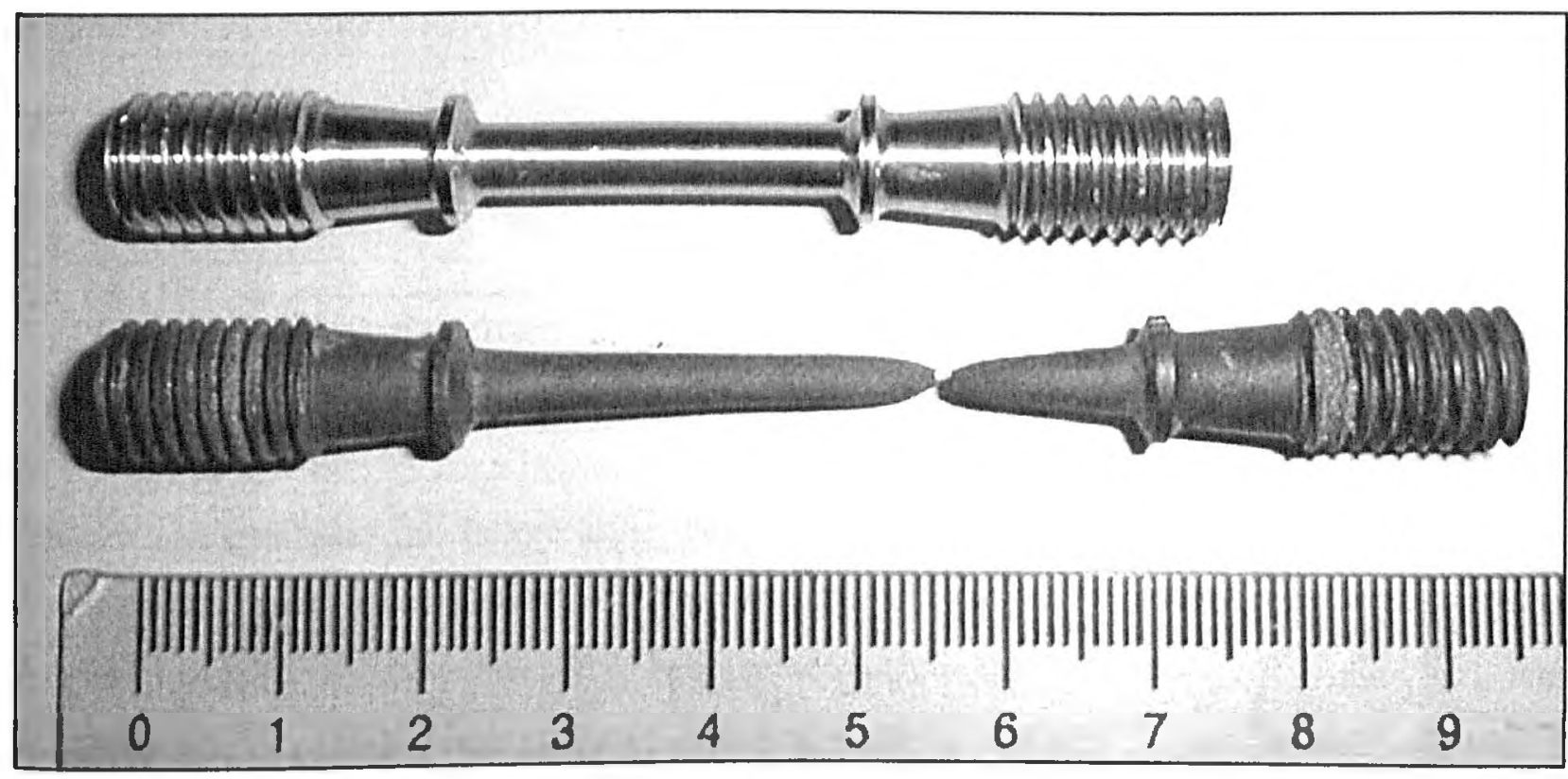

Figure 5.7: Top: Specimen before testing. Bottom: Uniaxial constant load creep specimen tested in air under a stress of 100MPa showing large amounts of ductility and necking to failure 

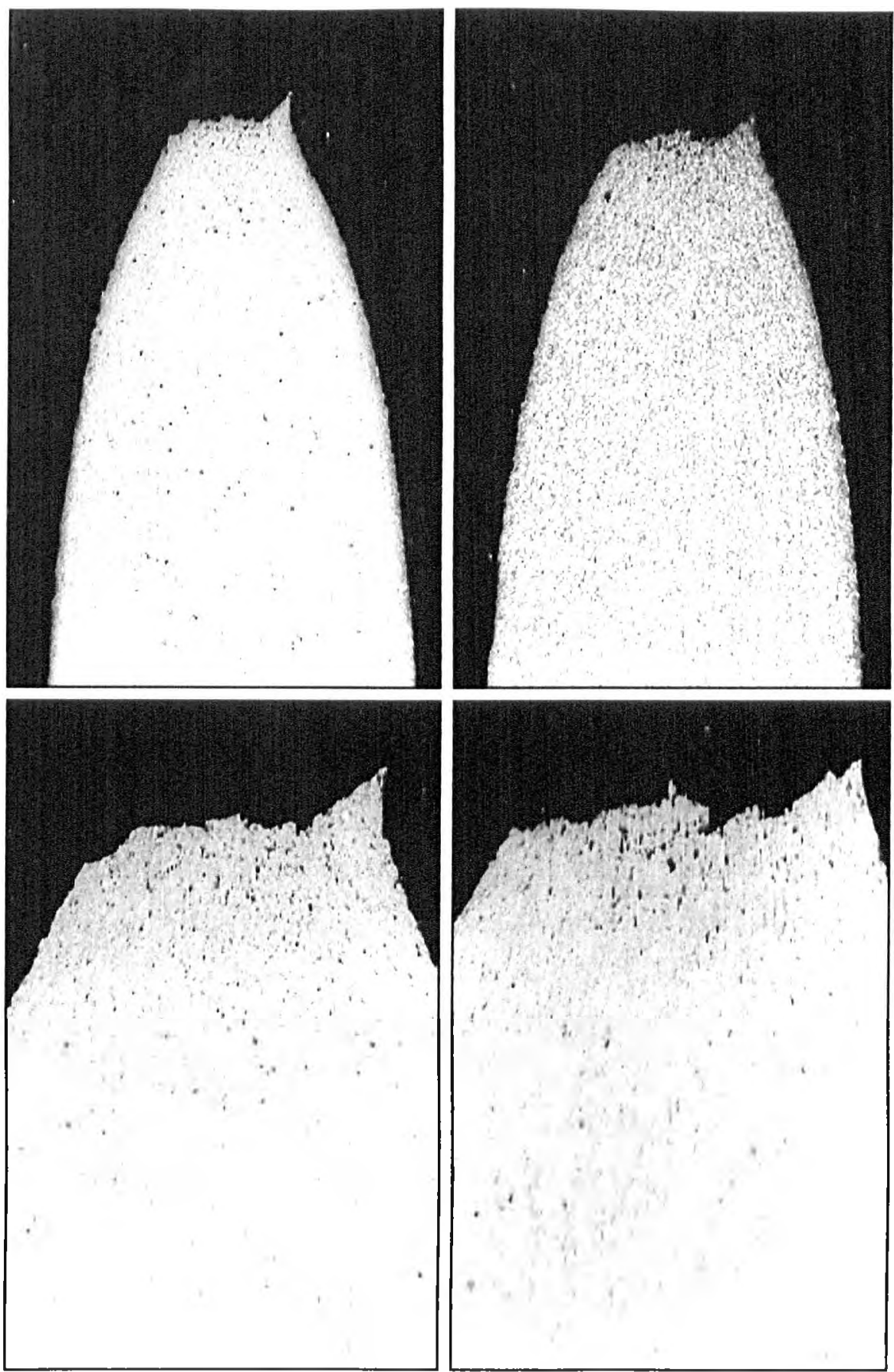

Figure 5.8: Optical micrographs of uniaxial creep specimens taken in the longitudinal direction (stress vertical). Left: specimen tested at llOMPa; Right: specimen tested at $90 \mathrm{MPa}$ (Magnification is $x 13$ Top and $\times 32$ Bottom)

cavities are seen near the failure zone, although these seem to be caused by the large local strains in this area. In addition, the only cavities seen in the bulk of the material tend to be at $\mathrm{MnS}$ inclusions which have cracked or become decohered during the creep life (figure 5.9). It is known that under longer testing times (i.e. lower stresses) this type of steel tends to undergo a transition to more brittle, grain boundary cavity dominated failure e.g.[Church'92], [Fields'80]. Importantly, under the given testing conditions the failure mechanisms appear to remain in the 


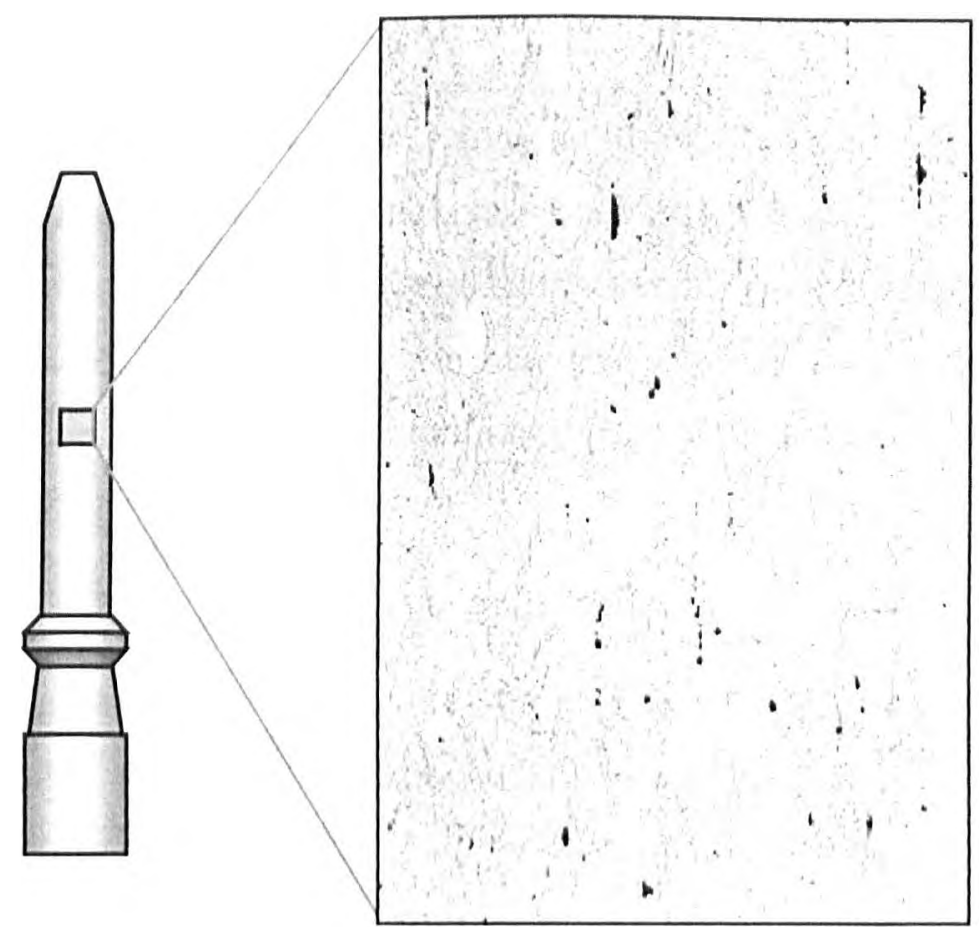

Figure 5.9: Typical damage in uniaxial creep specimens associated with decohesion and fracture of manganese sulphide particles (Mag. x160).

ductile regime. Therefore, any grain boundary cavitation seen in the work of this thesis is likely to be due predominantly to the presence of hydrogen, and not to the usual failure mechanisms of the given steel.

\section{Uniaxial Creep Test Summary}

The creep behaviour of the given material in air is characterised by high ductilities and relatively low strengths in comparison to the ISO standard. Failure appears to be due mainly to ductile thinning and tearing. There does not appear to be a lot of change in the failure mechanisms within the range of conditions studied. Failure times and ductilities are given in table 5.1.

\begin{tabular}{|c|c|c|c|}
\hline Stress [MPa] & $\begin{array}{c}\text { Failure time } \\
\text { [hours] }\end{array}$ & Failure strain [\%] & RA [\%] \\
\hline 110 & 209.7 & 60.4 & 91 \\
\hline 100 & 326.4 & 70 & 93 \\
\hline 95 & 470.8 & 59 & 94 \\
\hline 90 & 730.6 & 50 & 91 \\
\hline
\end{tabular}

Table 5.1: Summary of uniaxial creep test results in air 


\section{Argon Pressurised Tubes}

Three tubes were internally pressurised with $\arg$ on at $600^{\circ} \mathrm{C}$. The pressures used were 260,240 and 220bar, which correspond to mean diameter hoop stresses of 113.5, 104.7 and $96 \mathrm{MPa}$. Here, the mean diameter hoop stress has been used as an initial reference stress for the failure time of the tubes. Later, more accurate techniques will be discussed. Analysis of argon pressurised tubes allows the uniaxial and multiaxial results to be correlated, which will aid in future analysis of hydrogen pressurised tubes.

\section{Pressurised Tube Creep Curves}

The hoop strain of the pressurised tubes was measured in twelve locations with the capacitive displacement transducer described in section 4.3. Average hoop strains (based on the mean results from four gauges) were measured at the top, middle and bottom of the tube gauge length. Normally, maximum strain occurred near the middle of the tube, although on some tests the maximum strain was nearer the top. For clarity, the creep curves shown in figure 5.10 show only the maximum average strain measured.

The test at 240bar appears to have a much higher failure strain than the others. This test was allowed to creep until it burst open. Unfortunately, this type of burst failure caused damage to

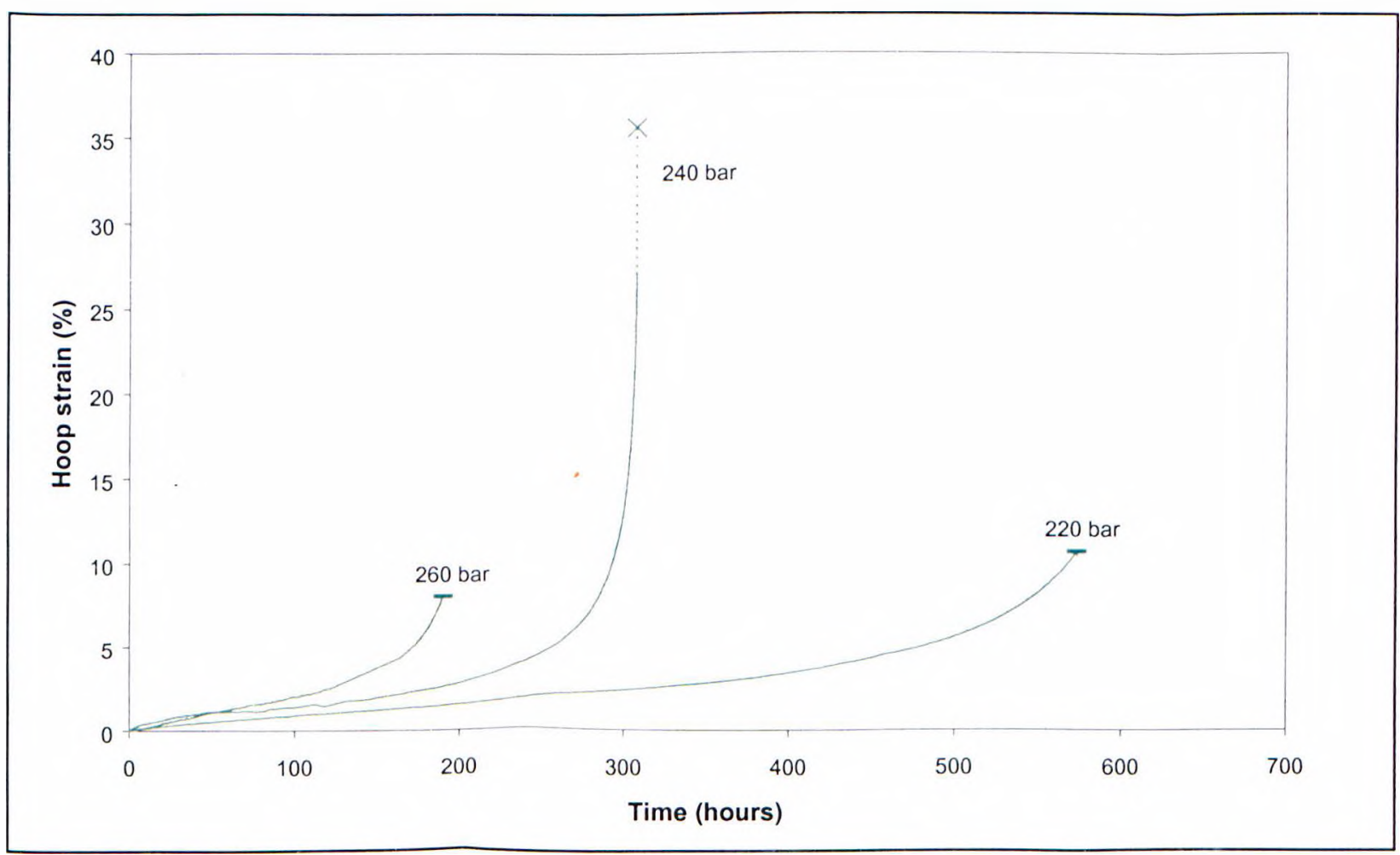

Figure 5.10: Maximum hoop strain vs time creep curves for argon pressurised tubes. The horizontal lines at the ends of the 260 and 220bar tests represent interruption of the tests to avoid bursting. Dotted lines represent strain to failure data was added manually after testing. 
the testing rig and, as a result, both the other tests were interrupted before bursting could occur.

The dimensions of these tubes were measured after testing with a micrometer from which strains were calculated. These strains were then compared to the strains measured by the capacitance gauges to allow for some evaluation of the extensometry. The results are given for both the argon and hydrogen pressurised tubes in Appendix B. The agreement between the capacitive measured strains and the micrometer results is very good, verifying the capacitance gauges and giving confidence in the results. The only exception is the $240 \mathrm{bar}$ argon pressurised tube which strained to a greater degree than indicated by the capacitance gauge. However, this was caused by the high strain rate at failure in combination with the insufficient frequency of the data-logging interval.

Figure 5.11 shows the pressure-rupture plot for the pressurised tubes. The exact failure time of the 260 and 240 bar tests is not know because they were interrupted at around $10 \%$ strain. However, examination of the creep curves in figure 5.10 suggests that interruption took place very close to failure. For example, at $10 \%$ strain, the tube tested at 240 bar was at $96 \%$ of its' time to failure. The rupture plot in figure 5.11 shows that all the data lie very close to a power law fit plotted through the data points. This suggests that the data set is consistent and can be used for comparison with other results in this thesis.

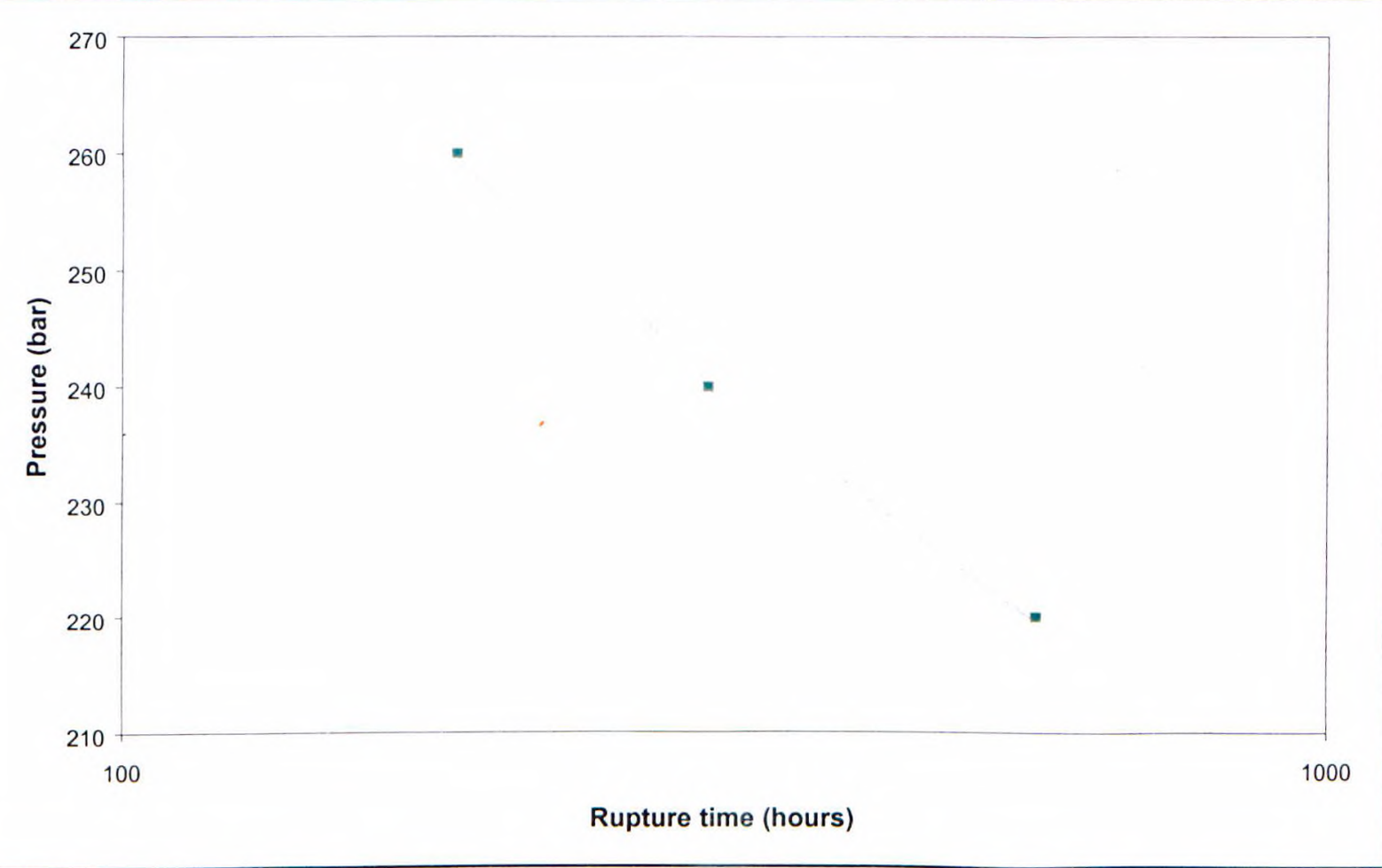

Figure 5.11: Pressure-rupture curve for argon pressurised tubes 


\section{Minimum Creen Rate}

The logarithm of the minimum creep rates of the pressurised tubes are plotted in figure 5.12 against test pressure. The ASTM seven point method could not be used to determine the minimum creep rate because there was too much noise in the early part of the creep curves. Instead, the rate was measured manually, and the error bars in figure 5.12 represent the uncertainty in the measured values. The pressure is not plotted in logarithmic scale to allow the data points to be seen better. The trendline is a best fit power law equation giving an ' $n$ ' value of 6.16 .

\section{Pressurised 7ube Ductility}

The tube burst at 240bar allows a good estimate of the material ductility to be made. Failure strain was relatively high at $35.6 \%$. In addition, wall thinning at the point of failure was up to $24 \%$. These numbers will be compared with ductility in hydrogen in section 5.3 . The other tubes also appeared to be ductile, with expected failure strains similar to the tube tested at 240bar. Photographs of the tubes after testing show some local bulging or non-uniform strain (figure 5.13). This bulging can be detected with the capacitance gauges. For example, figure 5.14 plots the average hoop strains in the top, middle and bottom parts of the specimen gauge length for the tube tested at $240 \mathrm{bar}$. The tube deforms uniformly until approximately 250 hours

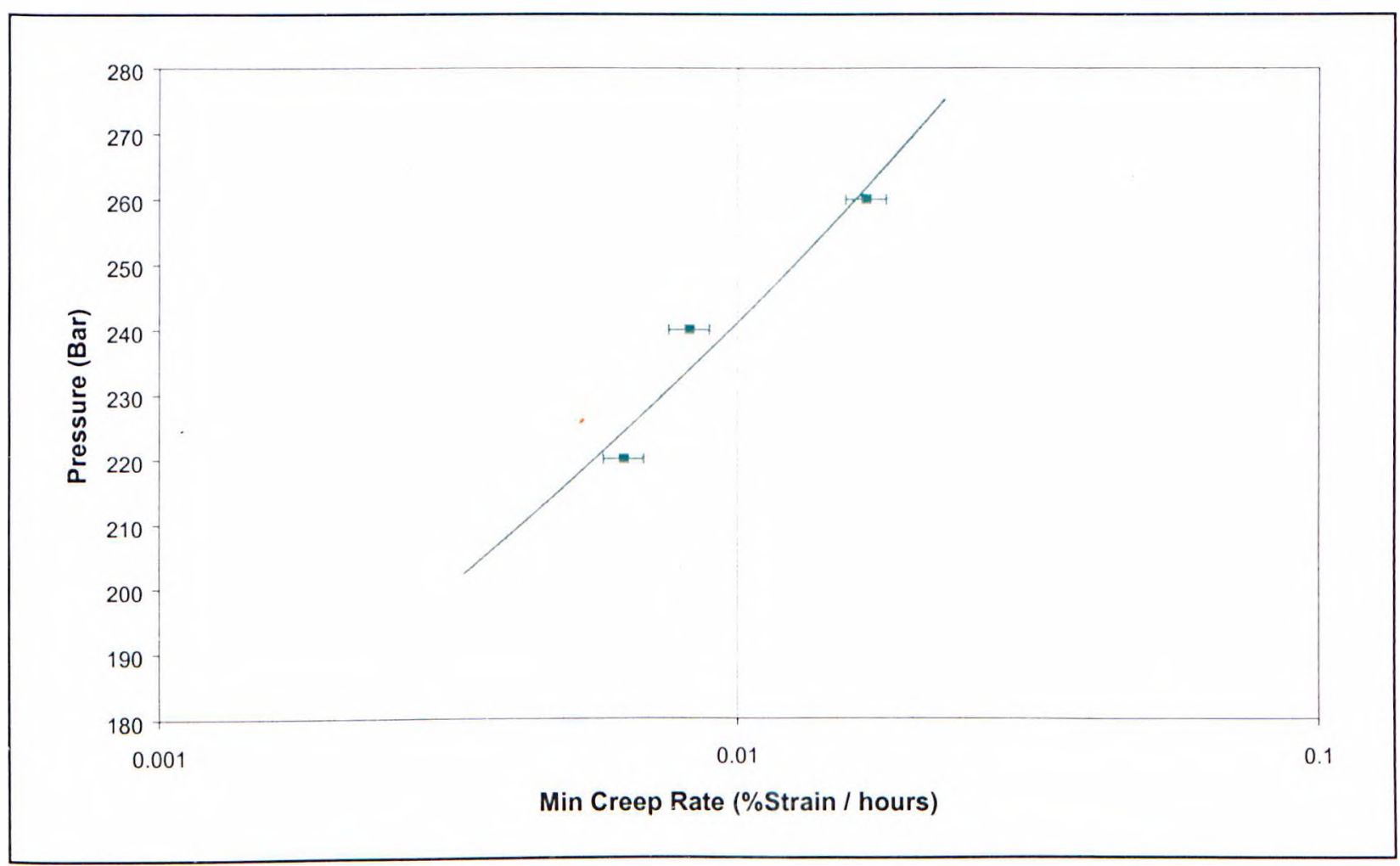

Figure 5.12: Log minimum creep rate vs internal pressure for argon pressurised tube tests. 
where a bulge begins to form between the middle and the top gauges. Thus, the stress redistribution and changes in geometry associated with bulging can be ignored for the majority of the lifetime.

\section{Metallography of Tubular Specimens}

Tubes for examination were sectioned both transversely and longitudinally. Normally, the first transverse section would be cut across the point of maximum strain, with additional sections being taken from other, less strained areas of the gauge length.

In general, metallographic examination of the tubes pressurised with argon reveals ductile behaviour. No cavitation is seen in any of the tubes, with the exception of the burst tube which showed small amounts of localised ductile voiding next to the fracture line. The burst failure occurred mainly as a result of local thinning followed by tearing of the remaining ligament. This thinning appears to have started from the outside of the tube wall and worked its way inwards (Figure 5.15). Examination of the transverse sections revealed some grain elongation in

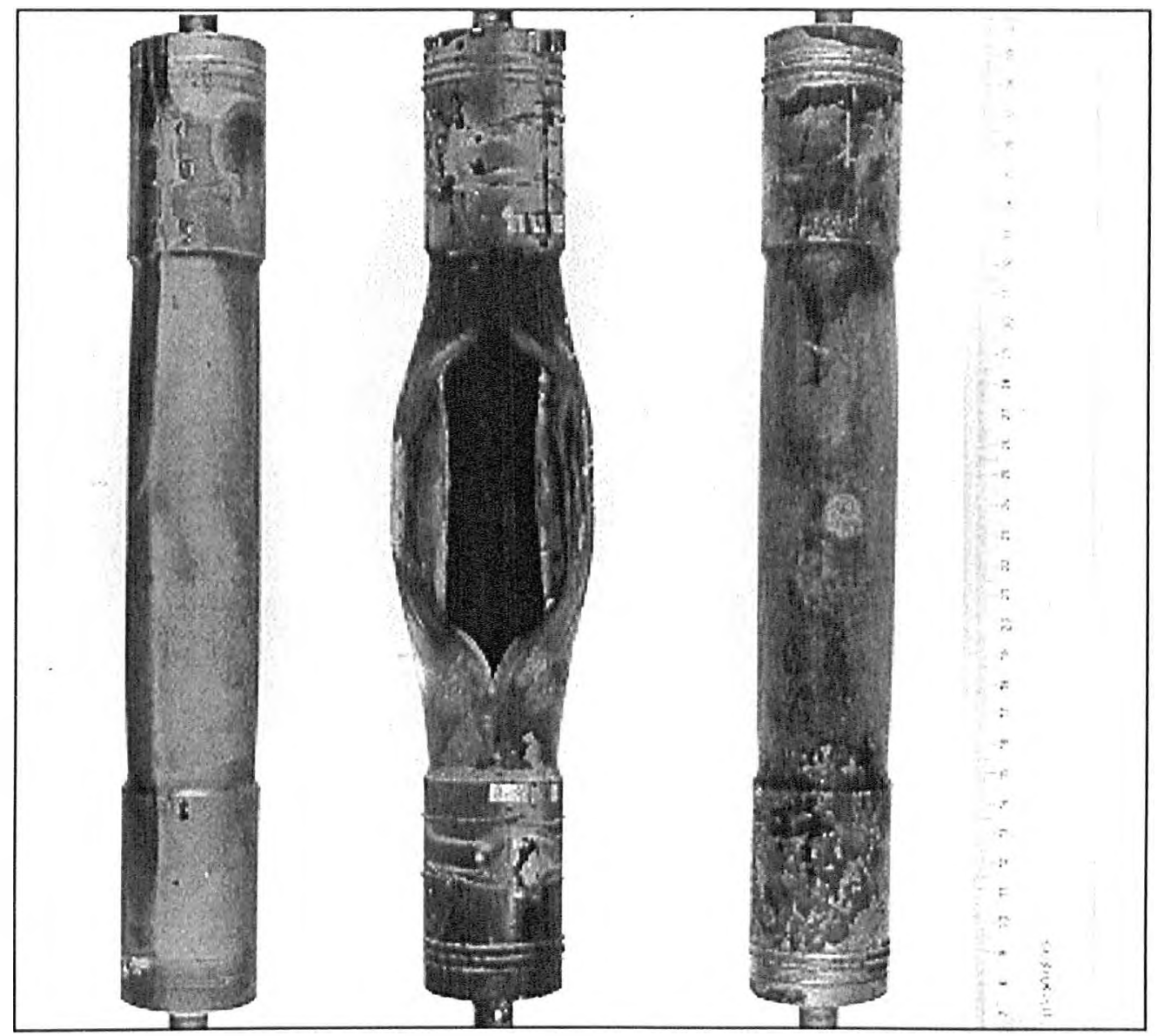

Figure 5.13: Post test photographs of tubes pressurised with argon (260 bar left, 240 bar middle, 220 bar right). Note the non uniform strain, particularly noticeable on the burst tube tested at 240 bar.(scale of ruler on right is $\mathrm{cm}$ ) 


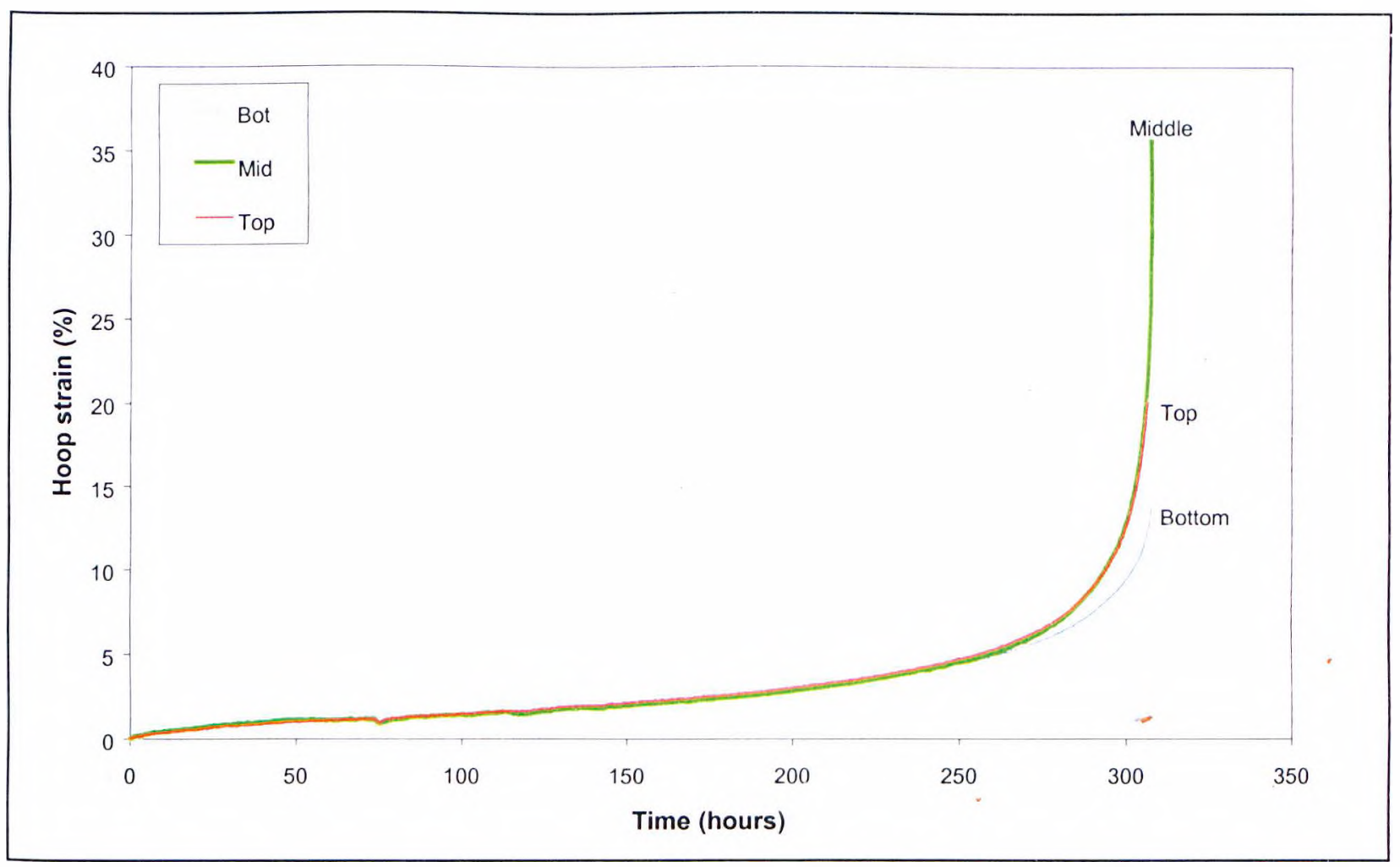

Figure 5.14: Average hoop strain measured at the middle, top and bottom sections of the gauge length of the tube pressurised with 240bar argon. Note uniform deformation until about 250hours where bulging begins to form between the top and middle gauges. Maximum failure strain is at the middle of the tube.

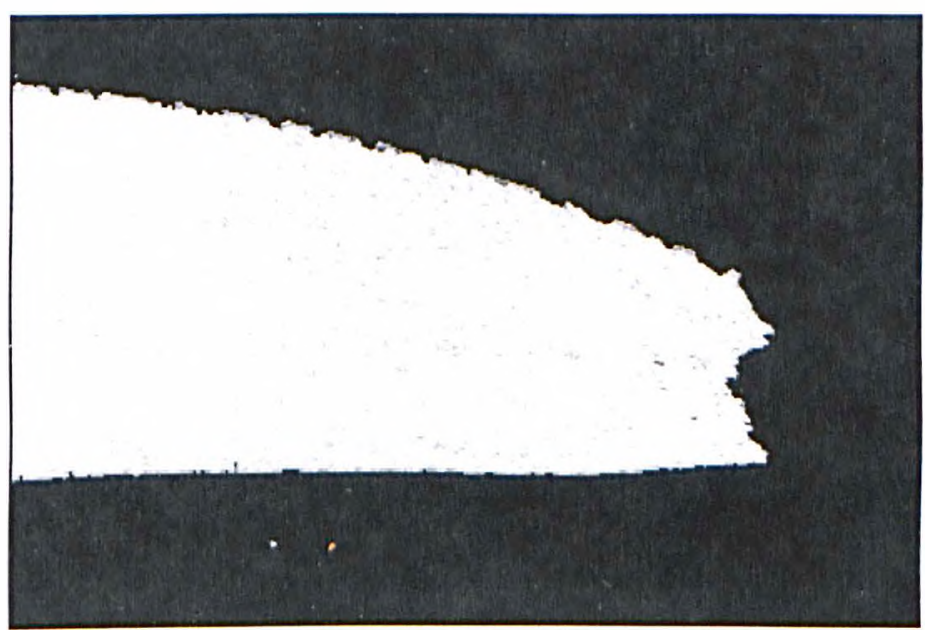

Figure 5.15: Transverse section of the failure in the tube burst with 240bar argon. Note the high ductility and thinning from the outside (Mag. x16)

the burst tube, however, elongation was harder to notice in the other tubes which had been interrupted at lower strain values (Figure 5.16).

Manganese sulphide particles were noticed in the material, with their length running parallel to the longitudinal direction. In this orientation, they look like small dots on the transverse 

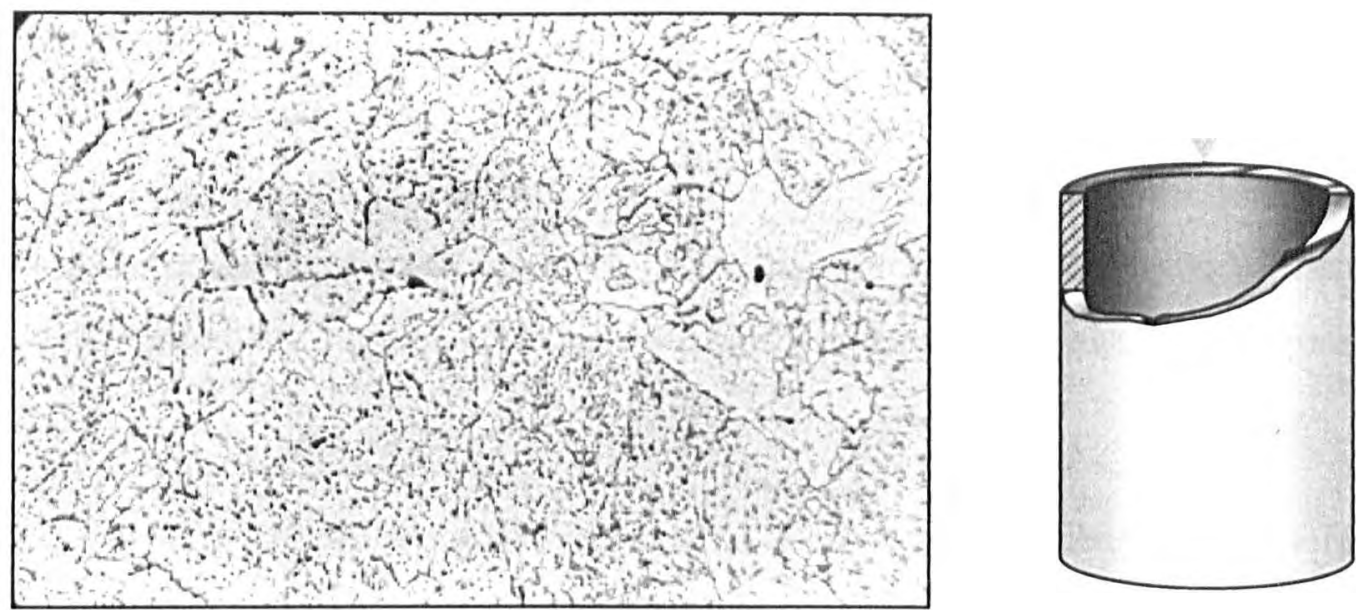

Figure 5.16: Transverse section at half wall thickness of the tube pressurised with 220bar argon. Hoop stress direction is horizontal. Note the lack of grain elongation and small undamaged MnS inclusions (Mag. x500).

sections, and do not appear to have been damaged by the hoop strain (Figure-5.16). Similarly, the longitudinal sections clearly show the MnS, again without any damage (Figure 5.17). This is in contrast to the uniaxial creep tests in air, where the MnS particles had generally fractured and had helped to form ductile voids. However, in the tube tests the strains were much lower, especially in the axial direction where the strain is approximately zero. In addition, the inclusions were aligned perpendicular to the hoop stress, which may have improved their resistance to cracking.
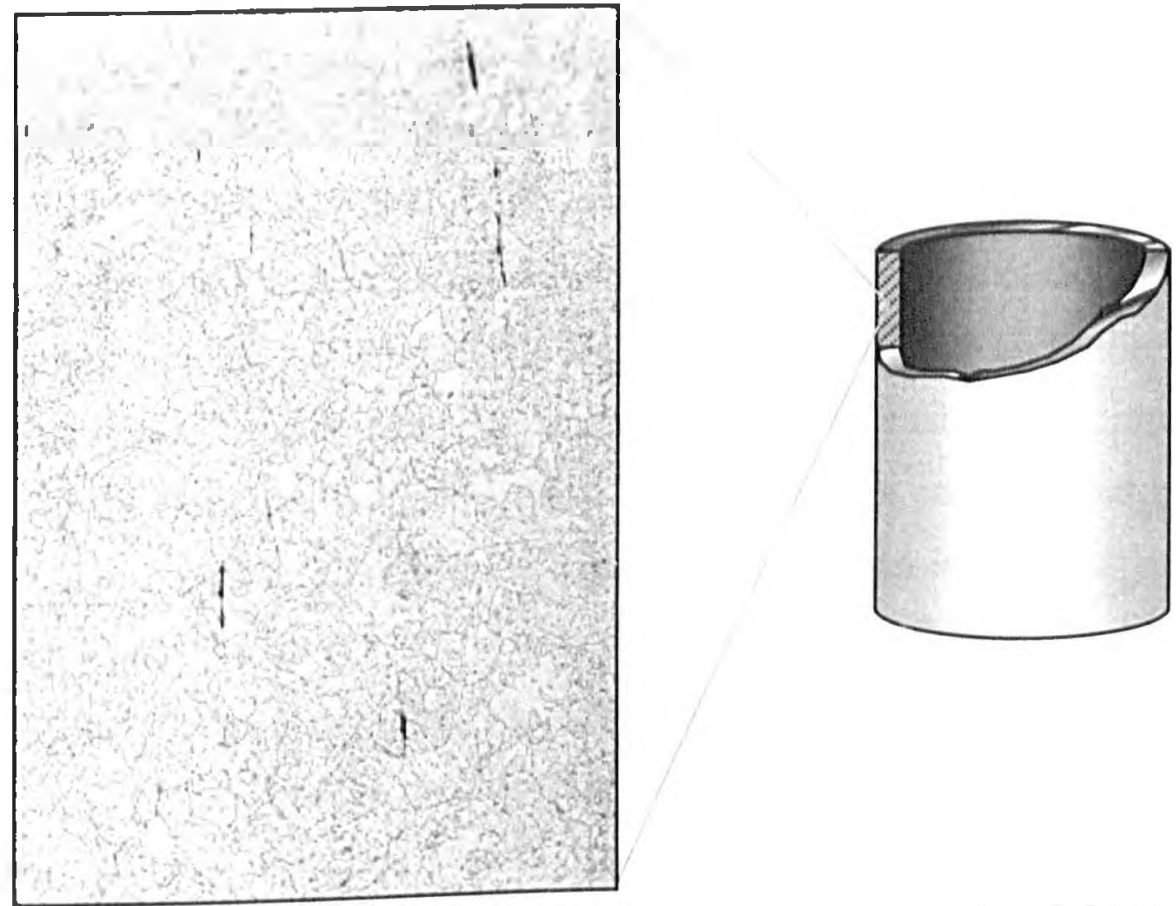

Figure 5.17: Mid-wall longitudinal section of the tube pressurised with 220bar argon, showing undamaged MnS inclusions parallel to the axial direction (Mag. x200) 
It is assumed that such inclusions had little influence of the creep behaviour in either uniaxial or pressurised tube tests, at least not until close to the end of life where they help to form ductile voids in the uniaxial specimens. They are mentioned here because any decohesion or fracturing would lead to the presence of small voids which could theoretically be nucleation points for the growth of hydrogen attack cavities when tests are performed under a hydrogen environment.

\section{Summary of Pressurised Tube Results}

All the tubes pressurised with argon showed ductile behaviour. Grain boundary cavitation was not observed, neither was void formation by decohesion or fracture of inclusions, except for very locally at the failure zone of the burst tube. Failure times and strains (or times/strains to test interruption) are given in the table 5.2 below.

\begin{tabular}{|c|c|c|}
\hline Pressure (bar) & Failure time (hours) & Failure Strain (\%hoop) \\
\hline 260 & $189.7^{*}$ & $8.0^{*}$ \\
\hline 240 & 307.4 & 35.6 \\
\hline 220 & $573.5^{*}$ & $10.7^{*}$ \\
\hline
\end{tabular}

*Interrupted test

Table 5.2: Argon pressurised tube test summary

\section{Correlation of Uniaxial Creep in Air, and Argon Pressurised Tubes}

\section{Rupture Correlation}

Rupture times of pressurised tubes are often estimated using a reference stress on the principal that a uniaxial specimen exposed to the same reference stress should fail in the same time. Two commonly used reference stresses are the mean diameter hoop stress and the skeletal point Von Mises stress [Cane'82b], [HTMTC'89]. When correlating internally pressurised tube behaviour, these stresses have little physical meaning, and are a rather empirical method of life prediction. The problem is worse when studying highly ductile materials (such as in the present work) because the high strains mean that the stress is ever increasing and rapidly becomes significantly different from the initial reference. This problem is compounded by the fact the stress increase for a given strain is different (faster) in tubes than in uniaxial specimens. One solution is to use characteristic stresses, which give a weighted average stress operating over the lifetime [Church'92]. However, the characteristic stress method requires constant stress uniaxial test data to be available. In the present work, there is the additional factor that the uniaxial tests were performed in air, and the tubes in argon. This can lead to errors if the amount of oxidation on the uniaxial specimens is great. For the relatively short testing times 
used, the oxidation did not appear to be significant, amounting to a layer only a few microns thick.

Given the above limitations, the tube rupture lives have been correlated using both the mean diameter hoop stress and the skeletal point Von Mises stress in figure 5.18 below. For these data, the mean diameter hoop stress gives the better correlation, with reasonable prediction of tube rupture lifetimes. This is in line with the work of Cane who suggested that under high pressures, tube lifetimes were best fitted with the mean diameter hoop stress [Cane'82]. The fit could be improved by choosing another reference stress (e.g Thick wall outer radius stress), or by using a rupture criteria based on a contribution of both hoop and Von Mises stresses as illustrated in section 2.6 [Hayhur.'84]. However, more accurate modelling of the creep behaviour will be carried out later in section 6, where prediction of the whole creep curve will be attempted, taking into account the stress increase with strain.

\section{Minimum Creep Rate Correlation}

Similar correlations to the rupture plot can be made for the minimum creep rates to determine how the principal strain in the tube can be predicted from uniaxial data alone. The resulting plot is shown in figure 5.19 for the mean diameter hoop stress and the skeletal point Von Mises

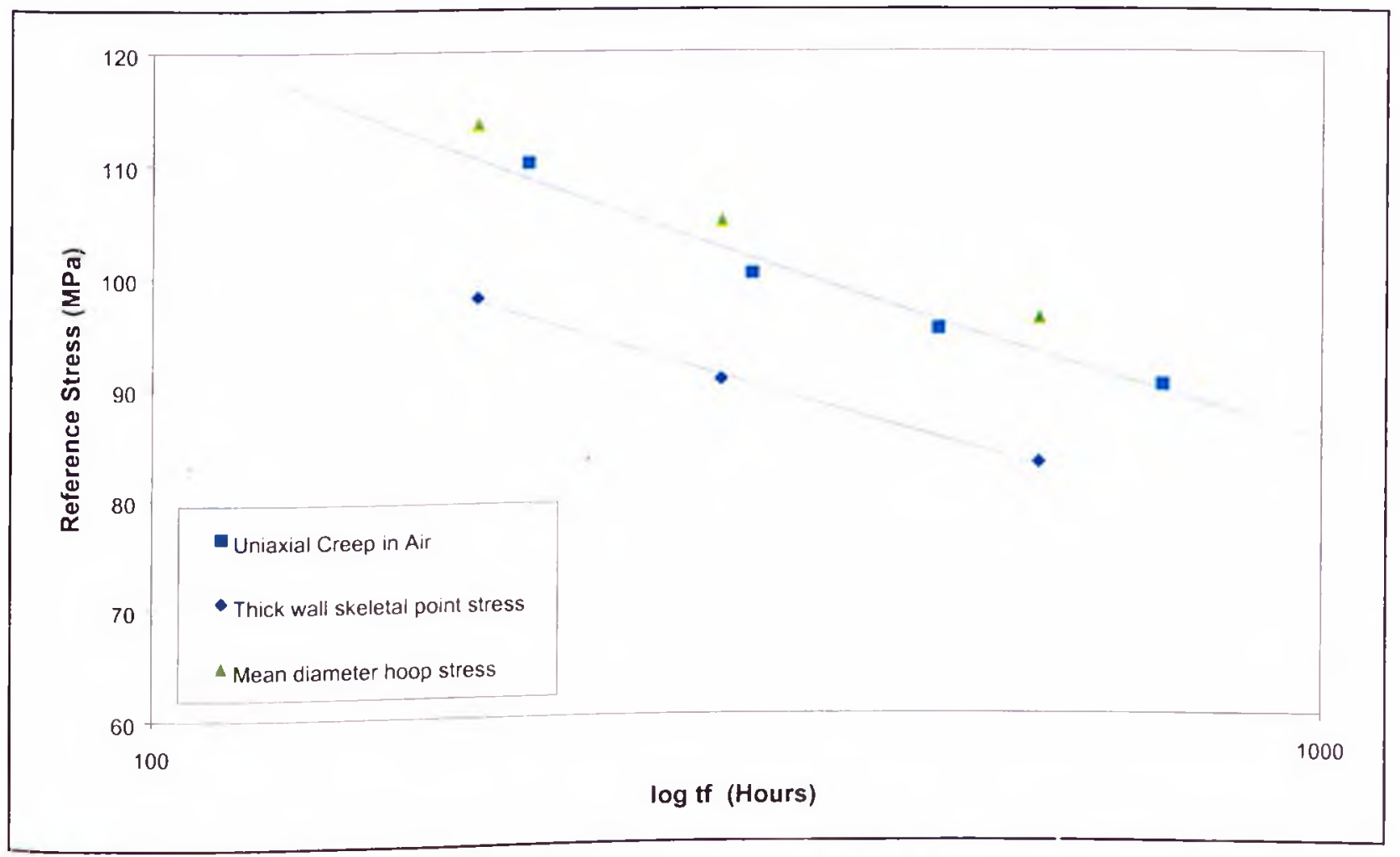

Figure 5.18: Correlation of pressurised tube ruphure data and uniaxial creep rupture using the mean diameter hoop stress and the skeletal point Von Mises stress. 


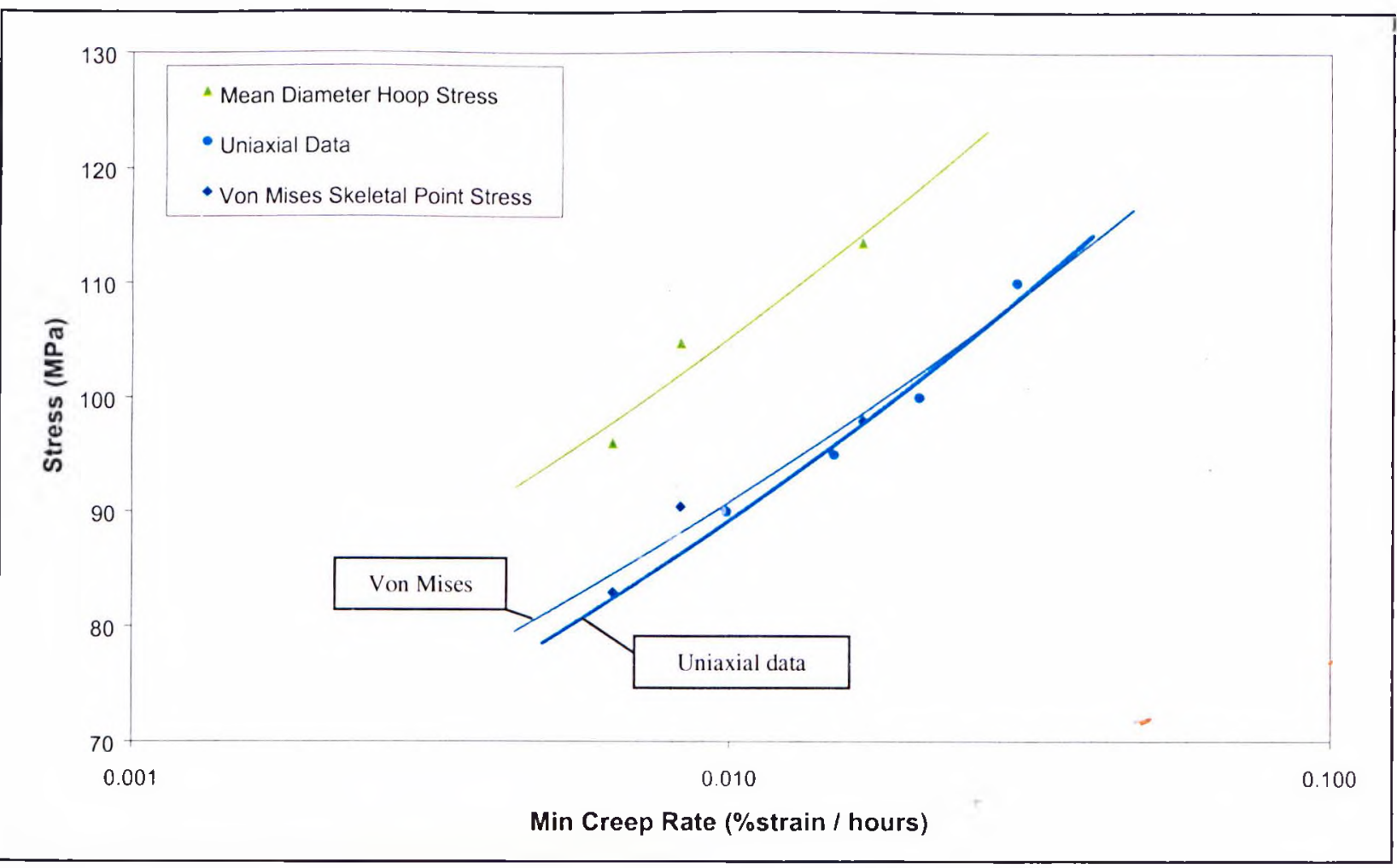

Figure 5.19: Correlation of minimum principal strain creep rate from argon pressurised tubes and uniaxial minimum creep rate using the mean diameter hoop stress and the thick wall Von Mises skeletal point siress.

stress. The skeletal point Von Mises stress clearly gives the better correlation and is often used in creep models to predict the strain rate behaviour e.g.[Church'92].

\section{Ductility Ratio}

Internally pressurised tubes normally have a failure strain somewhat lower that corresponding uniaxial test specimens due to the hydrostatic tensions which are present [Davis'59], [Manjoi.'75]. The reduction in ductility has been identified theoretically as ranging from 0.58 to 0.32 for a steel with an $n$ value of 8 , depending on which theory is used [Church'92]. For the results in this thesis, only the one tube which burst can be used for correlation purposes. The average failure strain for the uniaxial creep specimens was about $59.9 \%$. The burst tube had a failure strain of $35.6 \%$, giving a ratio of ductility of 0.59 which is close to one of the values above. The exact ratio found should not be given too much importance, especially given the wide range of theoretical values available and the averaging inherent in the measurement of hoop strain. It is perhaps more important to realise simply that the ductility has decreased by an amount which can be explained theoretically taking into account the multiaxial loading. 


\section{Metallographical Comparison}

Metallography from both uniaxial and tube tests confirmed very ductile behaviour. No significant difference was seen in the failure mechanisms between the two types of test, both showing large amounts of grain deformation leading to local necking and failure by local ductile voiding and tearing. The only difference was in the way in which $\mathrm{MnS}$ inclusions were damaged in the uniaxial specimens alone, although this may be due mainly to their orientation relative to the strain direction and the fact that uniaxial specimens strained to a greater extent than the tubes. 


\subsection{Combined Creep - Hydrogen Attack Behaviour}

\section{Uniaxial Creep in Hydrogen}

Four uniaxial constant load creep tests have been carried out within a 220bar hydrogen environment at $600^{\circ} \mathrm{C}$. The pressure was chosen as it was approximately the average pressure used in the internally pressurised tube tests (see later). Time restrictions meant that it was not possible to study the effect of hydrogen pressure in detail, so all of the tests were carried out under the same pressure (220bar). However, based on the Nelson curves the change in hydrogen attack with changing hydrogen pressure becomes small when the hydrogen pressure is greater than about 140bar. Furthermore, calculations of the methane pressure e.g. [VdBurg'96], show that the change in methane pressure with increasing hydrogen pressure is less pronounced when the hydrogen pressure is relatively high. Thus, it is ássumed that any errors caused by extrapolating the uniaxial data determined under $220 \mathrm{bars}$ to tubes pressurised with between 195 and 260bar should not be too large as a result of differences in hydrogen attack kinetics.

The stresses used in the hydrogen creep tests were subject to the frictional force mentioned in section 4. In addition, the load controller underwent a zero offset of approximately $100 \mathrm{~N}$ after the first two tests caused by drift. It will be shown in the following paragraphs how the error caused by these factors was minimised by performing a test with an inert environment in the hydrogen creep rigs (HCR) which could then be compared with the uniaxial data in air. Initially the applied loads were chosen to give similar stresses to those specimens tested in air.

\section{Determination of Applied Stresses}

It was calculated in section 4.2 that the frictional force on the HCR load train was approximately $-200 \mathrm{~N}$. In order to prove this, a specimen was tested in an argon environment in the HCR under an applied load of $1850 \mathrm{~N}$. Given the friction of $-200 \mathrm{~N}$, and the dimensions of the specimen, this corresponded to an estimated stress of 83.9MPa. The creep lifetime of this specimen was 1128hours. Assuming that the creep behaviour in air and in argon were the same, this data point was added to the rupture plot of the uniaxial creep tests in air. The resulting plot is shown in figure 5.20. The data from the specimen tested in argon sits on the rupture curve for tests in air. This verifies the assumption that the frictional force acting on the load train in the $\mathrm{HCR}$ is $-200 \mathrm{~N}$. Also shown in figure 5.20 are error bars on the argon data representing what the stress would have been if the friction was -100 or $-300 \mathrm{~N}$ instead of $-200 \mathrm{~N}$. 


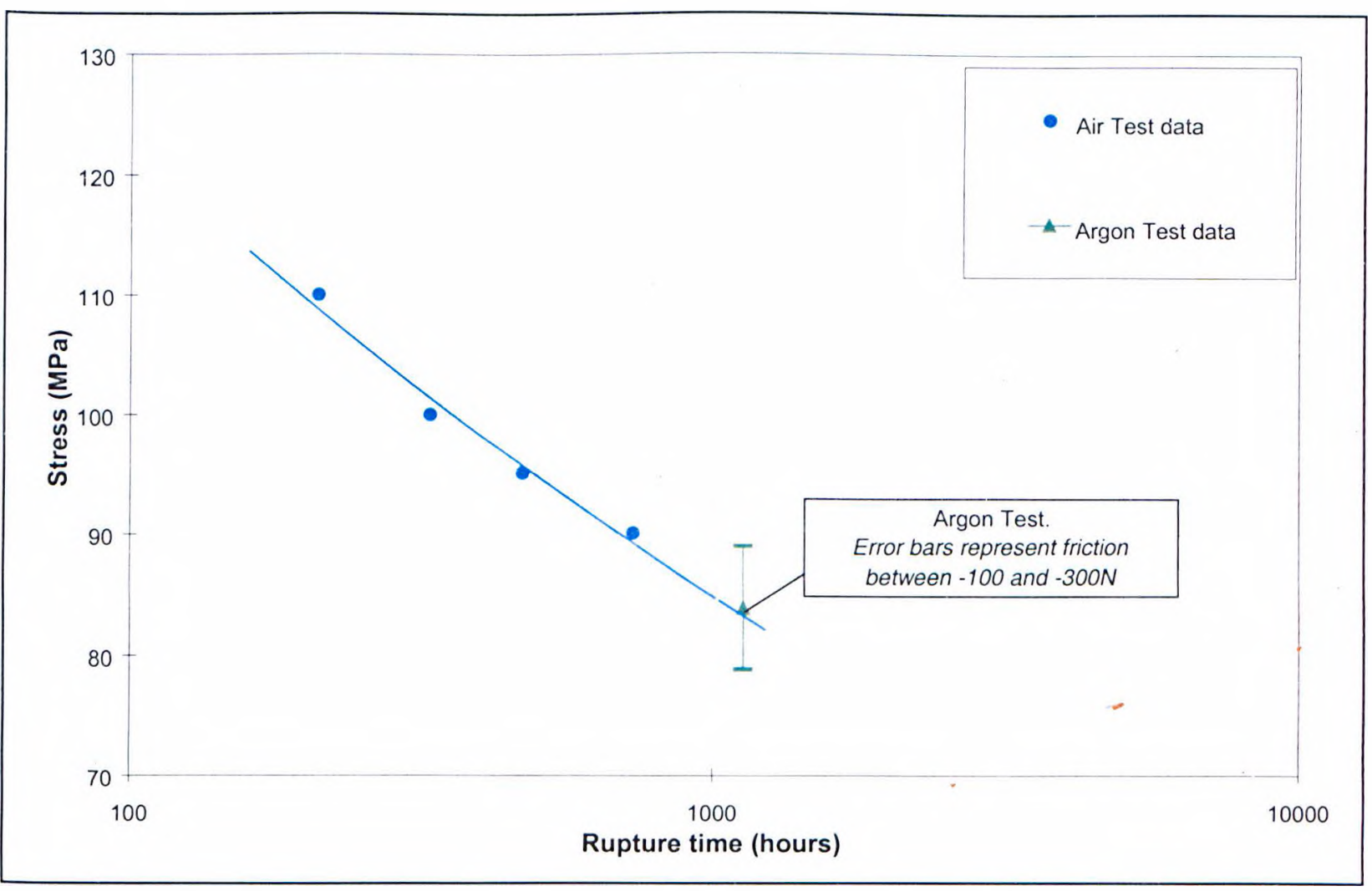

Figure 5.20: Stress rupture plot for uniaxial creep in air, showing the position of a test carried out in argon in the HCR whilst assuming a $-200 \mathrm{~N}$ frictional force. The position of the argon data point on the air rupture line verifies that $-200 \mathrm{~N}$ is the correct frictional force acting on the load train in the HCR.(error bars show the sensitivity to friction).

However, due to 'drift' of the loading mechanisms (possibly the electrical controllers), two of the tests in hydrogen still have some uncertainty in their stress values. The load offset was estimated at $100 \mathrm{~N}$, although it is expected that this value may be in error by up to $\pm 30 \mathrm{~N}$. As a result, plots containing data points from the two affected tests will be given error bars to represent this uncertainty, which amounts to approximately $\pm 1.5 \mathrm{MPa}$.

\section{Creep Curves in Hvdrogen}

Given the above considerations regarding the applied load, four tests were carried out under the following stresses: $104.9 \pm 1.5,94.9 \pm 1.5,86.8$ and $83.9 \mathrm{MPa}$. The corresponding creep curves are shown in figure 5.21. Also shown on the plot are the applied loads and any offsets used for calculating the stresses. It can be seen that the curves show little primary creep and that the strains to failure decrease with increasing test time.

On the 104.9 and the $94.9 \mathrm{MPa}$ curves, there are jumps in the creep rate occurring at about 75 and 220 hours. At these times, dumping of the active gas was triggered by an oxygen alarm in the bell housing. This occurred due to accidental depletion of the argon blanket gas supply. 


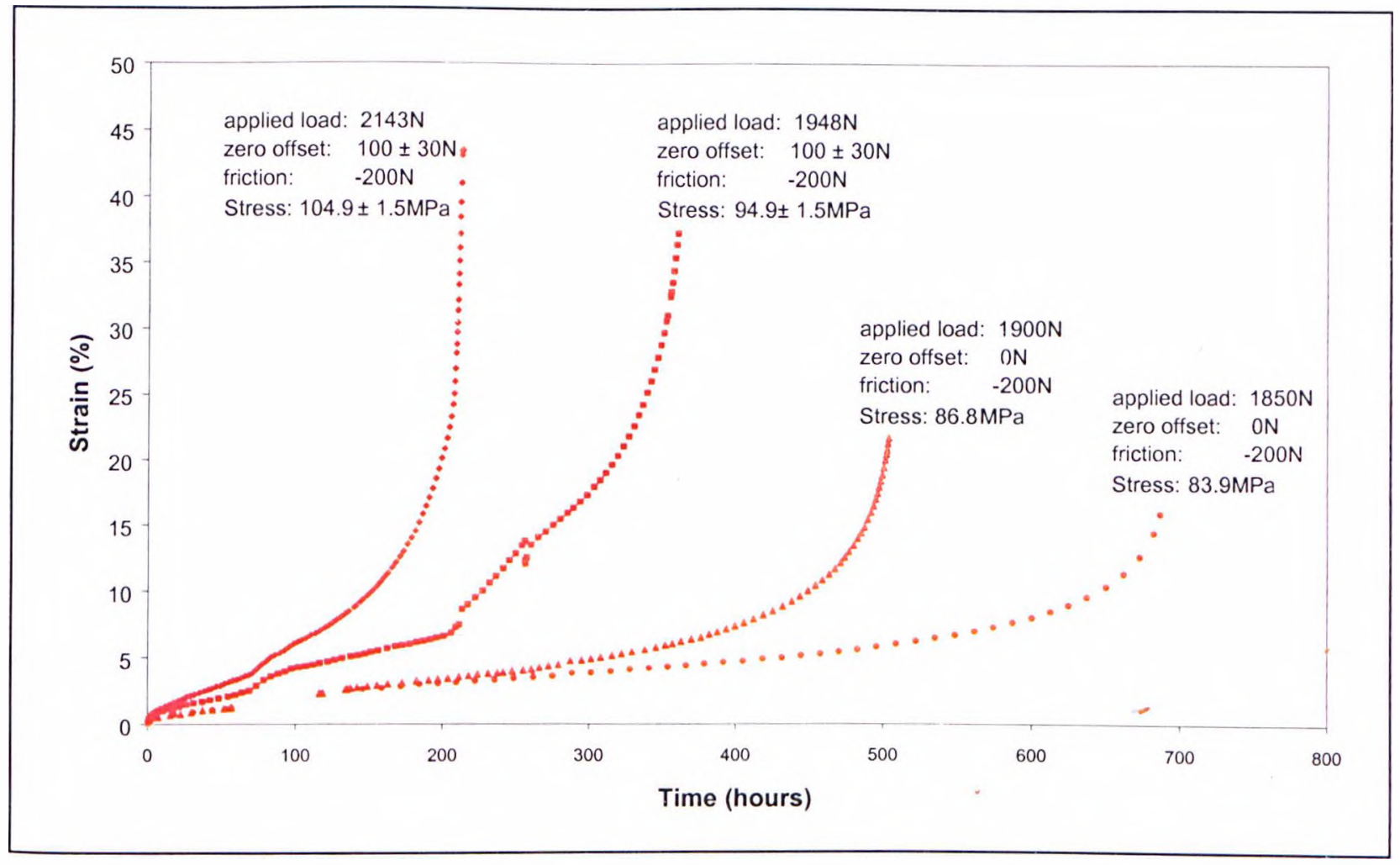

Figure 5.21: Constant load uniaxial creep curves for specimens tested under 220bar hydrogen at $600^{\circ} \mathrm{C}$

When the active gas is dumped, the frictional force exerted by the o-rings on the load train is reduced and, hence, the load on the specimen increases. This caused the creep rate to temporarily increase. The effect is most noticeable at about 220 hours on the 94.9 MPa curve. Thus, the failure time recorded for this curve may be slightly conservative, and the failure strain slightly excessive.

\section{Comparison of Creep Curves and Stress Rupture Behaviour With Air Tests}

Figure 5.22 plots the creep curves derived under both air and hydrogen conditions. In addition, the test carried out under an argon environment is also included. The first most obvious difference between the behaviour in air and hydrogen is in the strain to failure. The presence of hydrogen seems to have reduced the strains to failure, with the effect becoming greater as the test length is increased. It can also be seen that the rupture lifetimes are reduced in hydrogen. This is most noticcable when comparing the hydrogen and argon curves carried out under the same stress of $83.9 \mathrm{MPa}$. The change in rupture life is best observed however on the rupture plot in figure 5.23, where the decrease in rupture life under hydrogen is seen to be more severe under the longer term tests. This trend has not always been observed in the literature (see section 2.3 ). Thus, both the rupture life and the strain to failure are affected to a greater extent under longer term creep testing in a hydrogen environment. 


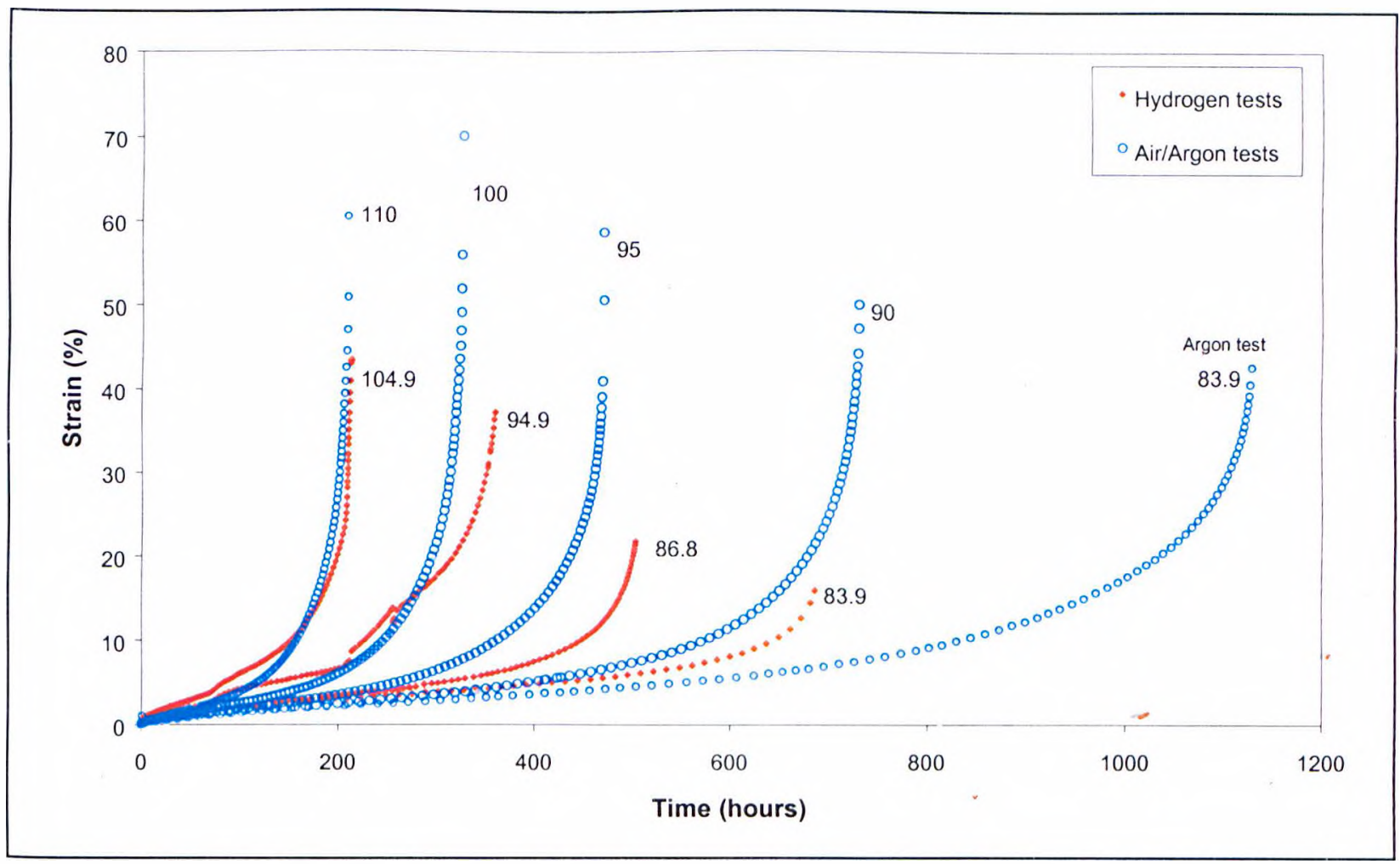

Figure 5.22: Uniaxial creep curves from both air/argon and hydrogen tests. The numbers next to the curves represent the applied stresses.

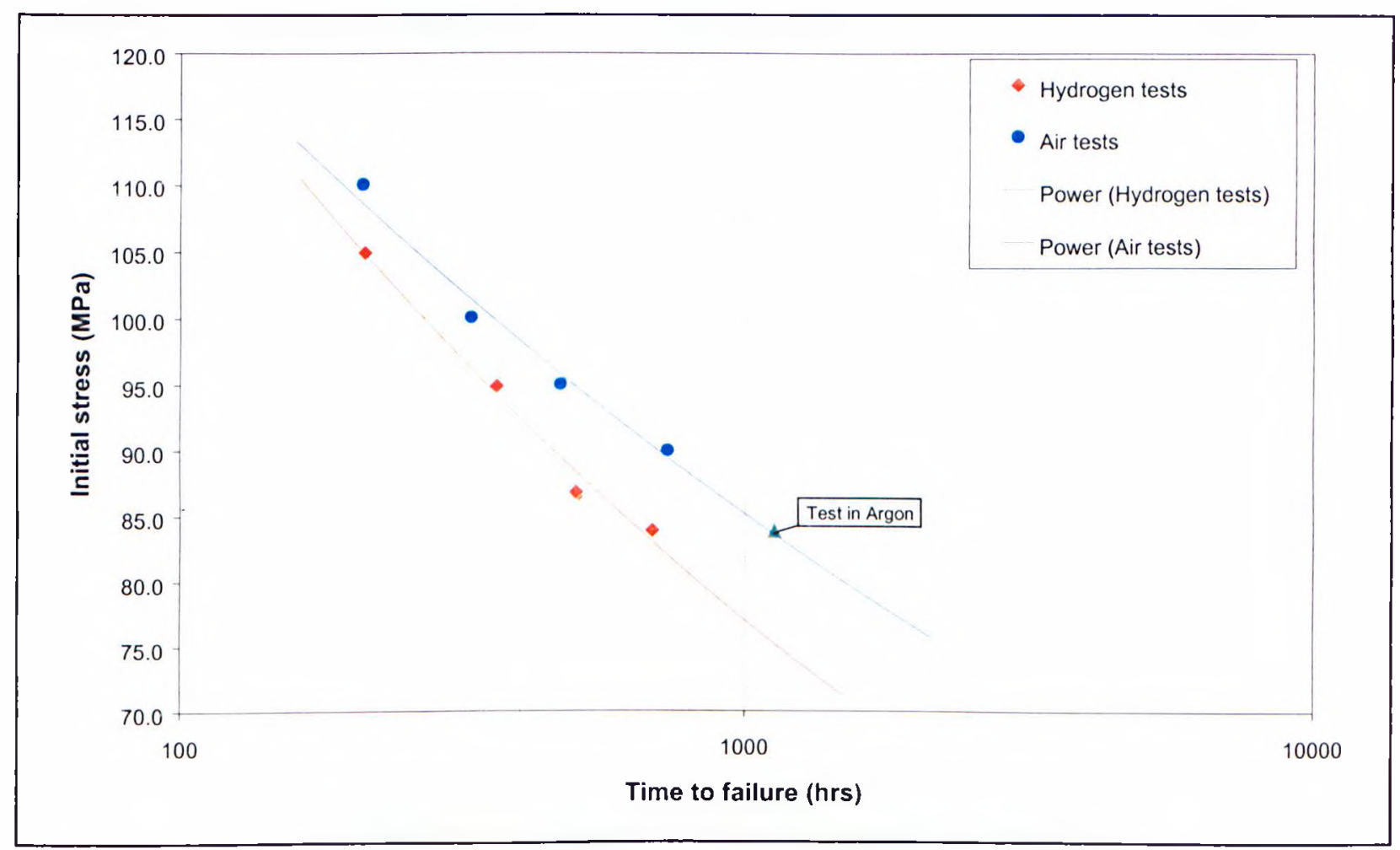

Figure 5.23: Comparison between the stress rupture plot for uniaxial creep in air and hydrogen. The plot has been extrapolated to show the increased effect of hydrogen at longer test times 


\section{Minimum Creep Rate in Hvdrogen}

The minimum creep rate was calculated from the creep curves and is plotted against stress in figure 5.24. Also included in figure 5.24 is the minimum creep rate data for uniaxial creep in air. The hydrogen tests show a consistently higher minimum creep rate for a given stress and also a slightly higher ' $n$ ' value (6.26 in hydrogen compared with 5.46 in air). The error bars represent the uncertainty in the manual measurement of the creep rates. The effect of hydrogen is to increase the minimum creep rate.

\section{Creep Ductility in Hvdrogen, and Comparison with Air}

The decrease in elongation with decreasing stress can be seen in figure 5.25, which shows a collection of photographs taken of the different specimens after failure. In addition, the amount of reduction of area (RA) can be seen to decrease with decreasing stress as well. Table 5.3 shows the values of strain to failure and reduction of area for creep tests in hydrogen. The effect of stress on the creep ductility is best seen in figure 5.26 where plots of strain to failure and RA are made against stress for both air and hydrogen tests. In addition, the figure shows that the ductility in hydrogen is significantly lower than for similar stress tests in air. Not only are the RA and strain to failure reduced in hydrogen, but they also exhibit a greater dependency on the

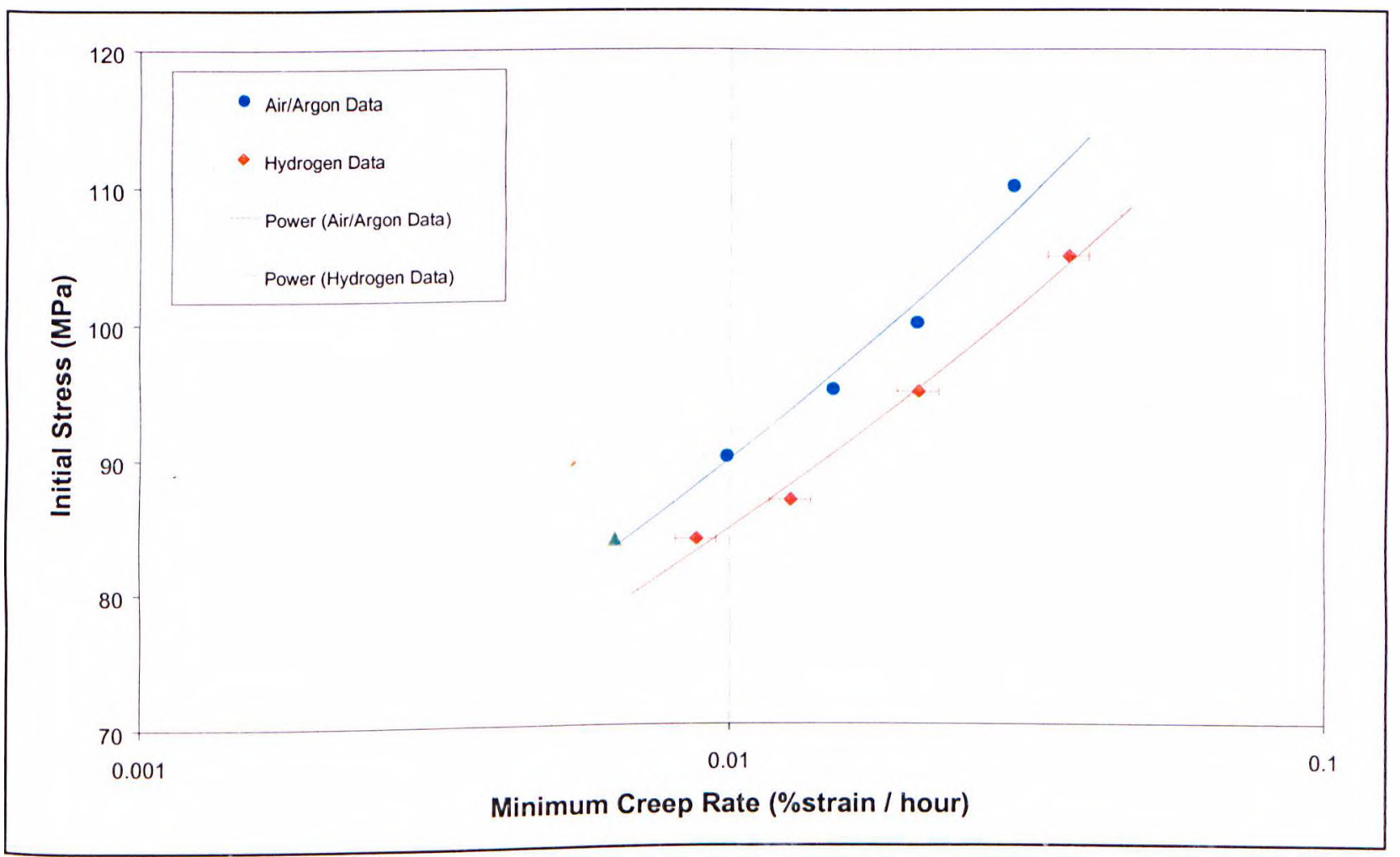

Figure 5.24: Minimum creep rates vs stress for hydrogen and air tests. Power law trendlines are also shown on the plot. Error bars represent the uncertainty in the measurement technique. 


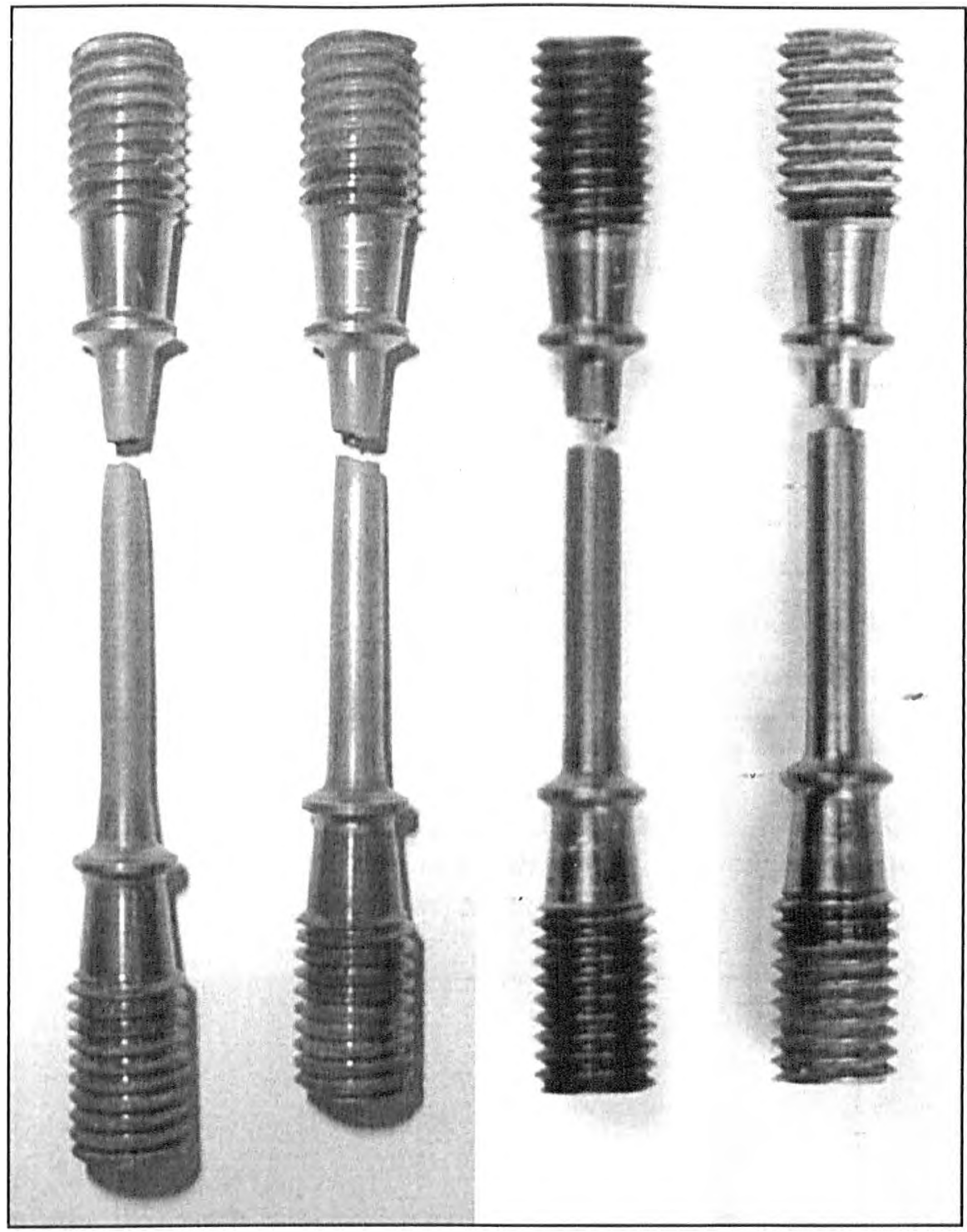

Figure 5.25: Uniaxial creep specimens tested in 220bar hydrogen under stresses of: (from left to right), $104.9 \pm 1.5,94.9 \pm 1.5,86.8$ and 83.9MPa.

test stress. For example, in air, the RA is more or less constant for all stresses. However, as the stress decreases, the RA for specimens tested in hydrogen decreases too. The reduction in the strain to failure with decreasing test stress is also more pronounced in hydrogen than in air. These results confirm similar observations from the literature, eg. [Norris'76], [Yokaga'84], [Ciuffr.'82]. The effect of hydrogen on ductility for a given stress is illustrated photographically by comparing the test specimens tested in argon and in hydrogen at $83.9 \mathrm{MPa}$ in figure 5.27.

\begin{tabular}{|c|c|c|}
\hline Stress (MPa) & RA (\%) & Strain to Failure (\%) \\
\hline 104.9 & 63 & 44 \\
\hline 94.9 & 40 & 37 \\
\hline 86.8 & 26 & 21 \\
\hline 83.9 & 15 & 16 \\
\hline
\end{tabular}

Table 5.3: RA and strain to failure for uniaxial creep specimens tested in hydrogen 


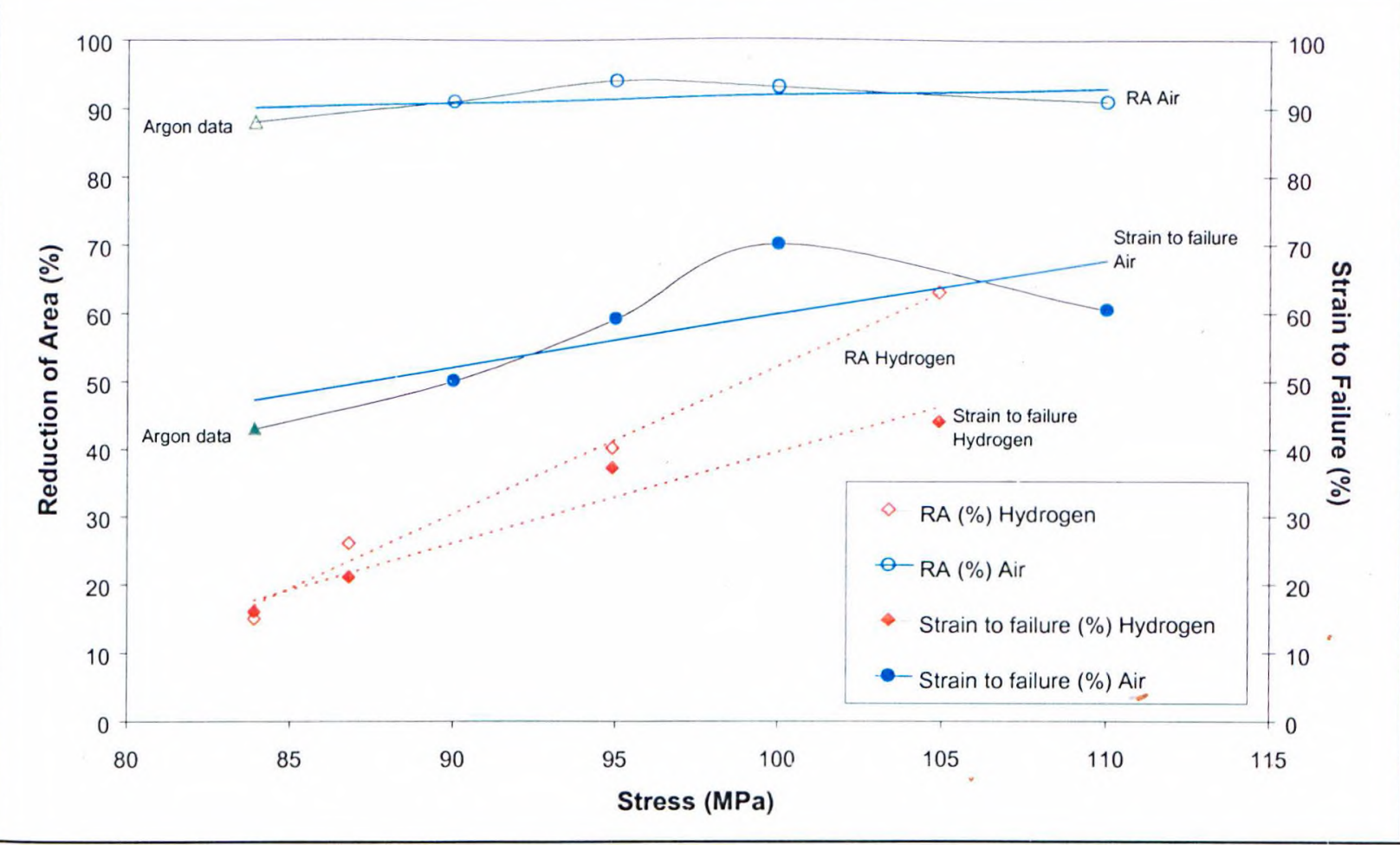

Figure 5.26: The effect of test stress on the strain to failure and reduction of area for uniaxial creep specimens tested in air and in hydrogen.

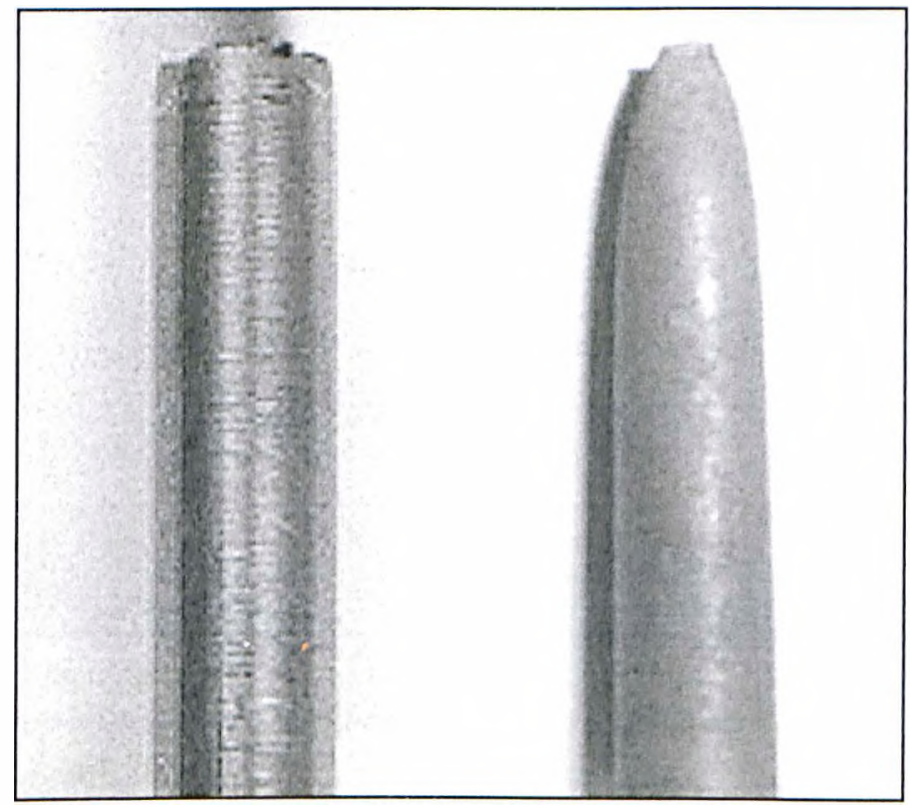

Figure 5.27: Close up comparison of the failure zones of creep specimens tested at 83.9MPa in hydrogen (left) and argon (right). Note the loss of ductility in the hydrogen test.

\section{Metallographic Examination of Uniaxial Hvdrogen Tests}

Each of the four specimens were sectioned both longitudinally and transversely. Examination of the longitudinal sections reveals extensive cavitation, predominantly on grain boundaries. The growth and subsequent link-up of these cavities is what leads to the reduced rupture lifetimes 

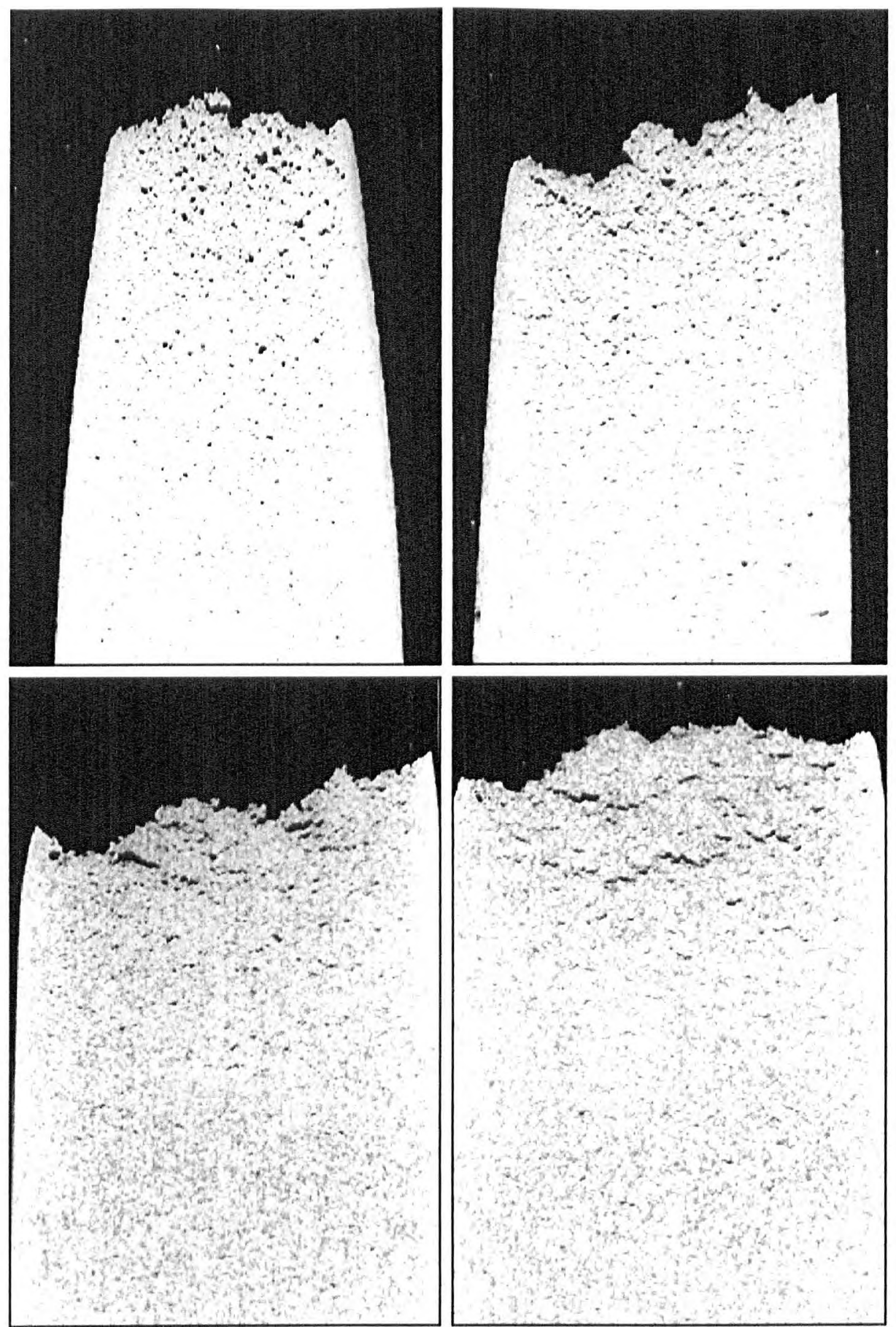

Figure 5.28: Low magnification (x13) optical microscopy photographs of longitudinal sections of the creep specimens tested in hydrogen. (Top left: 104.9MPa (212.9hrs); top

right: $94.9 \mathrm{MPa}$ (363.5hrs); lower left: $86.8 \mathrm{MPa}$ (503.2hrs); lower right: $83.9 \mathrm{MPa}$ 1686.2hrs]). Note the decrease in ROA with decreasing stress and the increased tendency for orientation of microcracks at $90^{\prime \prime}$ to the stress.

and ductilities. Such cavities are characteristic of hydrogen attack. However, the cavity morphologies and distributions vary between the specimens. Under the lower stress (longer time) tests, there is a trend towards preferential cavitation on those grain boundaries oriented perpendicular to the stress (figure 5.28). In addition, lower stresses produce small, crack-like 


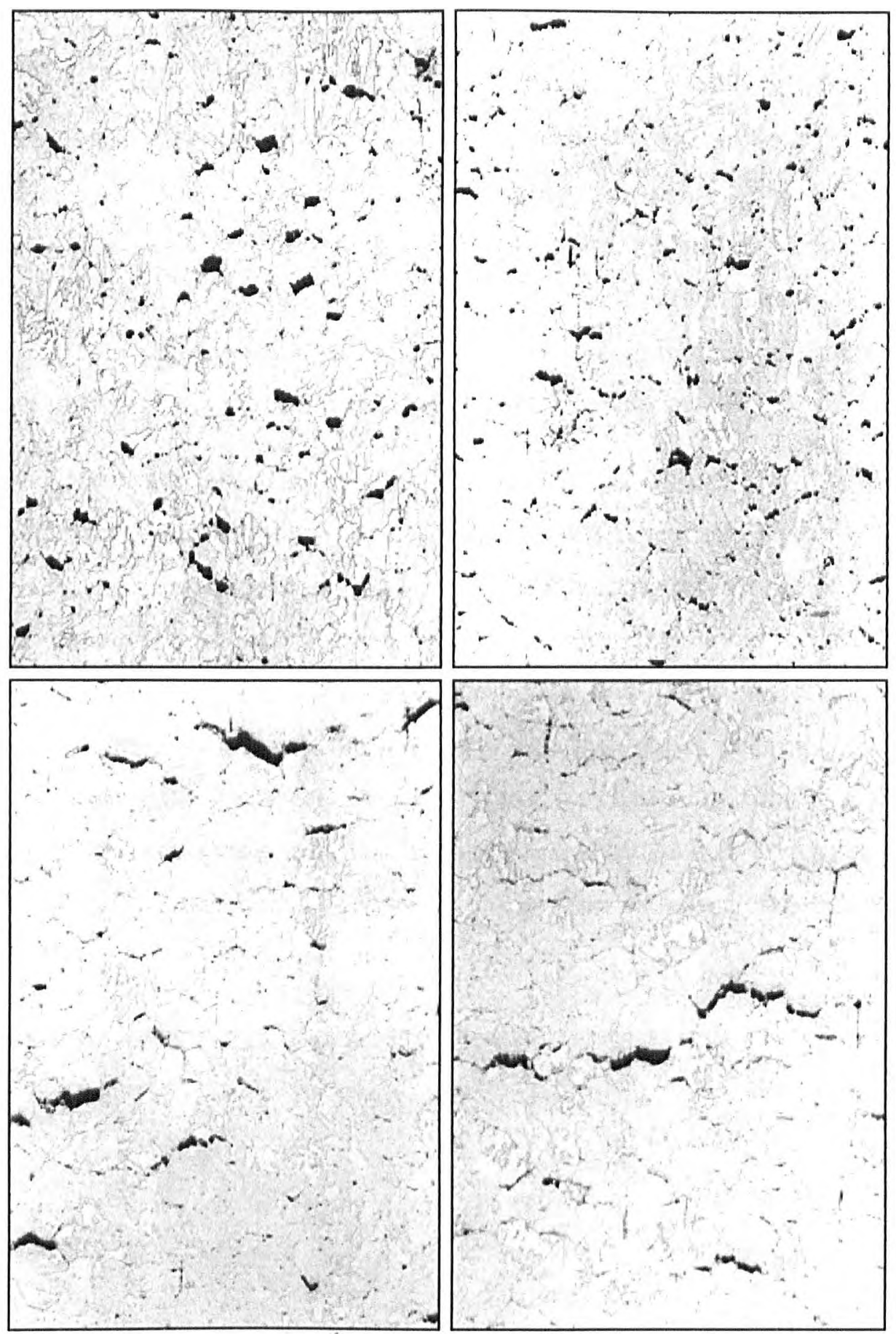

Figure 5.29: Close up (x130) optical microscope view of the cavities from longitudinal sections of the creep specimens tested in hydrogen. (Top left: 104.9MPa (212.9hrs); top right: $94.9 \mathrm{MPa}$ (363.5hrs/; lower left: $86.8 \mathrm{MPa}$ (503.2hrs); lower right: $83.9 \mathrm{MPa}$ (686.2hrs)). Note the change in cavity morphology and alignment with decreasing stress, where the stress direction is vertical. (Lightly etched with $5 \%$ Nital)

cavities, whereas higher stresses produce more rounded cavities scattered homogeneously throughout the material (figure 5.29). This change in cavitation is matched by a change in creep behaviour, with the lower stress tests producing more brittle ruptures. 
The tendency towards crack like voids at lower stresses is in contrast to the work of Chuang et al., who predict that under certain conditions cavity growth will become more crack like in the presence of high applied stresses [Chuang'79]. They argue that under higher stresses the grain boundary diffusive flux increases so that the rate of surface diffusion within the cavity controls the growth rate. When this happens, there is not enough time to form the equilibrium rounded cavity shape by surface diffusion, and the crack-like form predominates. Clearly then, a different mechanism is taking place in the current work. To attempt to clarify the situation, the SEM was used to look closer at the cavities in the creep specimens.

The SEM reveals that the crack like voids are in fact caused by the growth and link up of a large number of small grain boundary cavities (Figure 5.30). The actual shape of the individual cavities is in fact rather rounded. Under higher stress, the number of very small voids appears to be diminished relative to the lower stress case. Grain boundarics may have several cavities on them, yet they are unable to link-up so easily as under low stress because they are further spaced from each other. As a result, the cavities in the high stress material must grow much larger before they can coalesce which gives the material a chance to strain to a greater degree (Figure 5.31). Under low stress conditions, the grain boundary cavities are closer together, and can link up to form long microcracks relatively easily. Hence the material behaves in a more brittle manner.
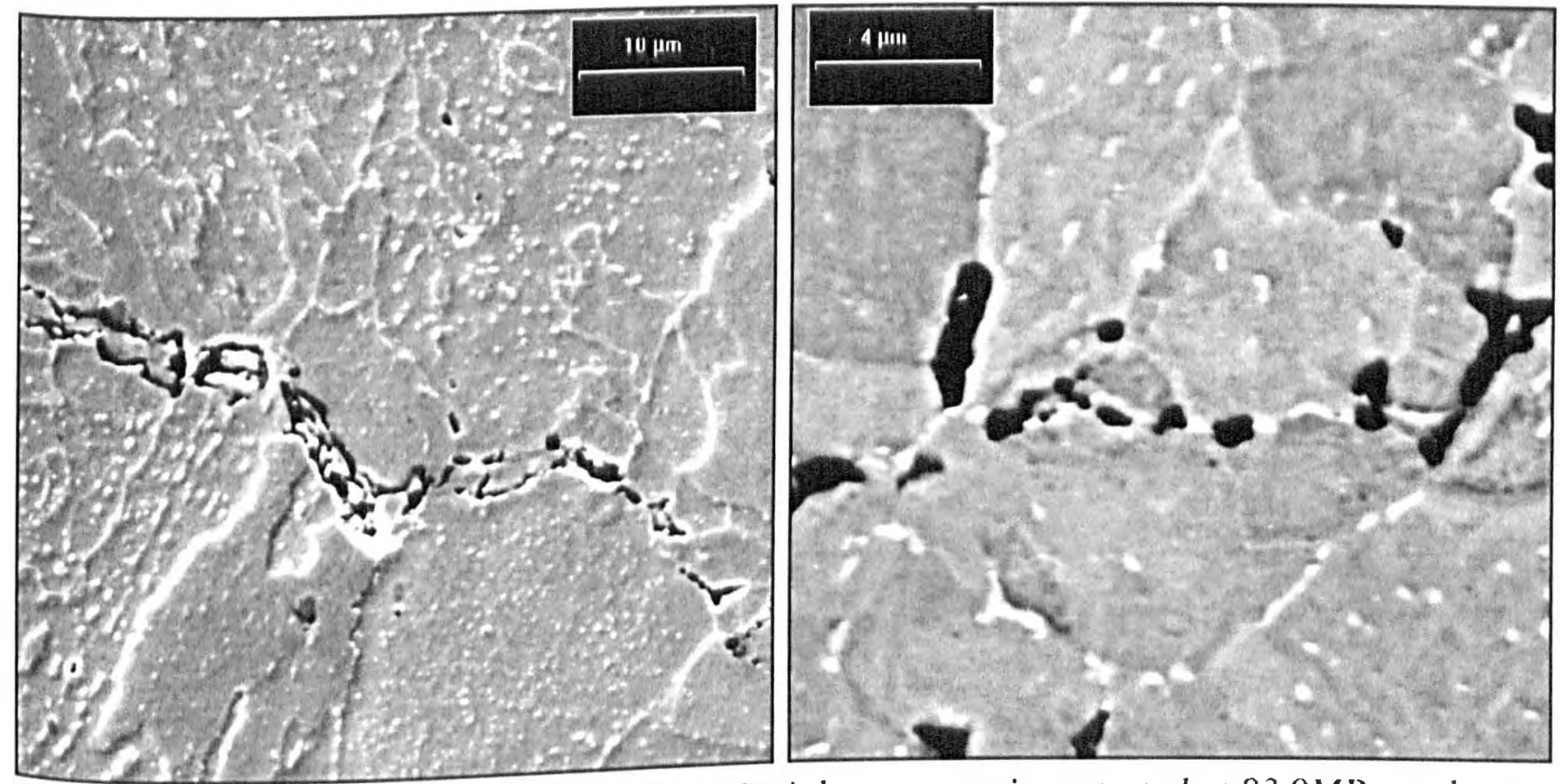

Figure 5.30: SEM micrographs of the uniaxial creep specimen tested at 83.9MPa under 220 bar hydrogen at $600^{\circ} \mathrm{C}$ for $686.2 \mathrm{hours}$, where the stress direcition is vertical. Showing rypicai grain boundary microcrack (left) caused by growth and link-up of small, individual grain boundary cavities (right) 


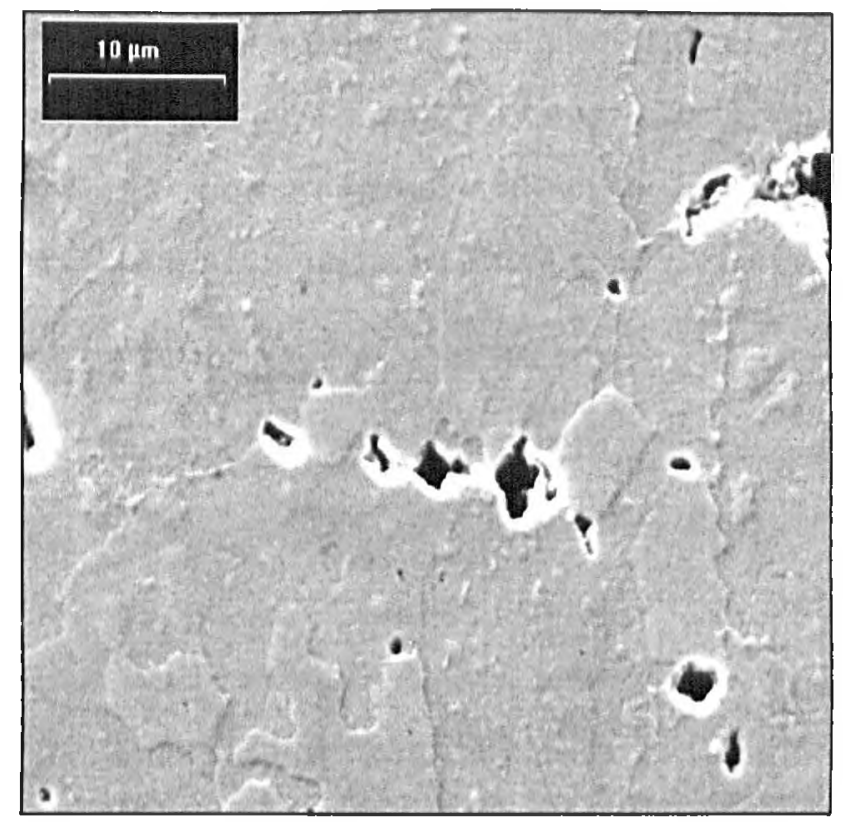

Figure 5.31: SEM micrograph from the specimen tested at 104.9MPa under 220bar hydrogen for 212.9 hours at $600^{\circ} \mathrm{C}$ (stress direction vertical). Note the relatively high spacing of the voids and their size attained before link up (cf figure 5.30)

The uniaxial creep specimens were also sectioned longitudinally through the shoulders. The thinnest part of the uniaxial creep specimen shoulders was about $6 \mathrm{~mm}$ diameter tapering up to about $9 \mathrm{~mm}$ next to the screw threads. Evidence of cavitation was barely visible in the specimen shoulders, in both the thickest and the thinnest sections, independent of the test stress. The small size and the sparse distribution of these cavities meant that it was not possible to observe any trends between the different specimens, or within the tapering shoulder itself.

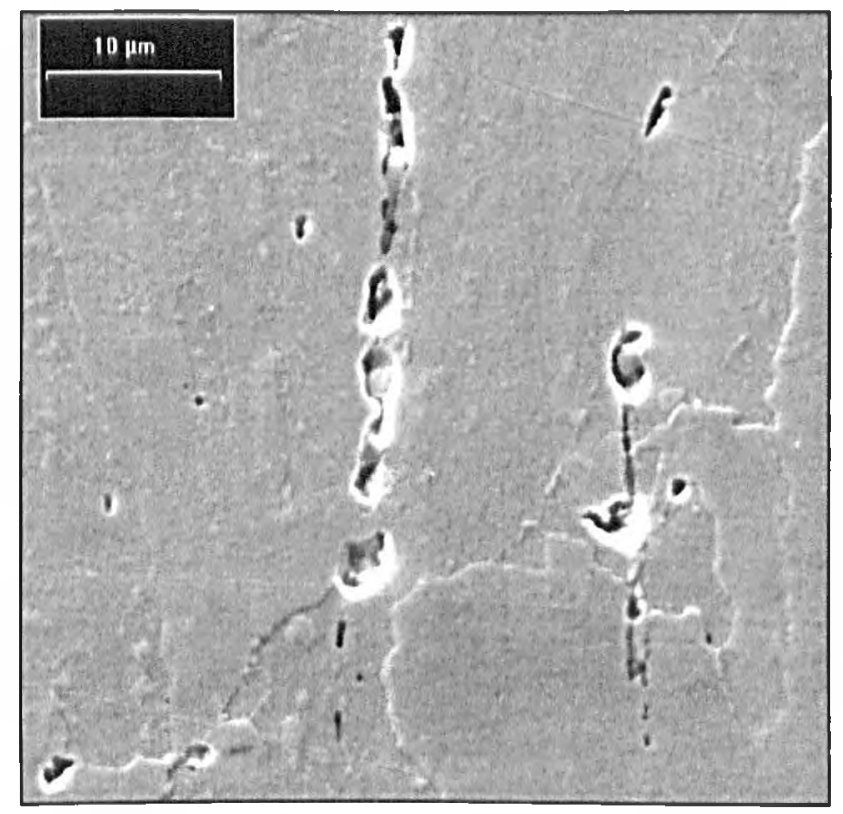

Figure 5.32: Hydrogen attack cavitation at MnS inclusions in the uniaxial creep specimen tested under 104.9MPa (stress vertical) 
Figure 5.32 shows cavitation around manganese sulphide particles aligned parallel with the applied stress. Cavitation of this sort was commonly observed, particularly in the higher stress tests where the $\mathrm{MnS}$ inclusions had often fractured. However, fracture appeared to be controlled mainly by cavitation situated on grain boundaries oriented at $90^{\circ}$ to the applied stress.

\section{Surface Decarburisation in Creep Specimens}

Surface decarburisation was observed in the creep specimens. The observable depth at failure ranged from about $100 \mu \mathrm{m}$ in the shortest term test to about $200 \mu \mathrm{m}$ in the longest test (N.B. there is in fact a gradient of carbon content from the surface inwards, so defining a depth to which it is apparent is subject to error). Cavitation was greatly reduced in the decarburised zone. This is to be expected as surface decarburisation and cavitation are competing processes [Rosent.'63]. As decarburisation proceeds, there is less carbon available in the surface area to form methane internally in voids, and thus cavitation is reduced. Figure 5.33 shows the lack of voids near the surface for the shortest and longest term tests. The decarburisation itself is not
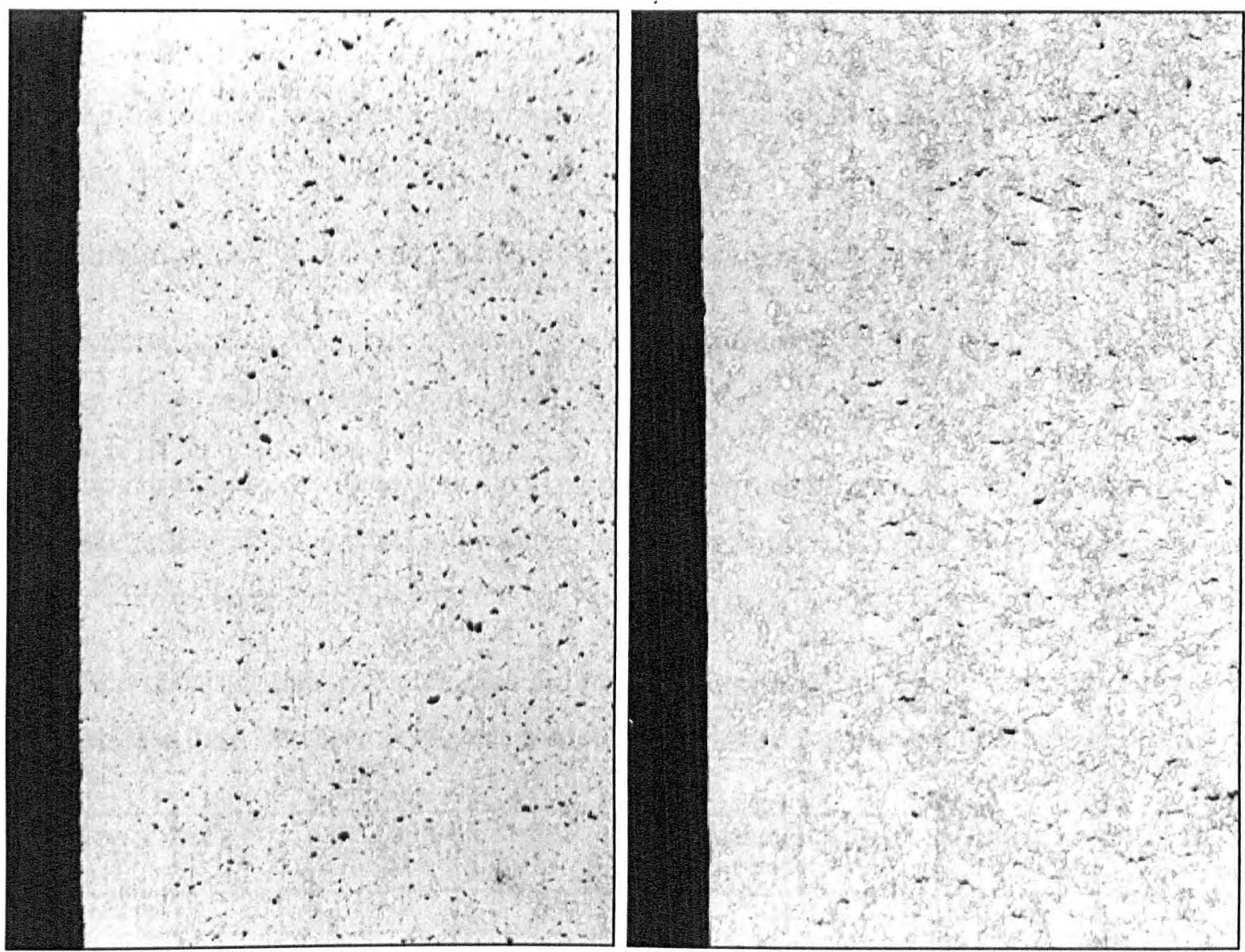

Figure 5.33: Surface decarburisation and the resulting loss of cavitation in uniaxial creep specimens tested in hydrogen. Left: tested at 104.9MPa for 212.9hours, Right: tested at 83.9MPa for 686.2hours. (Mag. x80) 
very evident in the photos due to the minimal etching which was performed to avoid corrosion and enlargement of the voids.

The decarburised zone has lower strength than the bulk material due to the lack of carbon. In industrial situations, this can normally be ignored due to the relative size of the decarburised layer compared with the thickness of the component. However, for small laboratory creep specimens operating at elevated temperatures, the depth of decarburisation could theoretically become sufficient to change the overall creep strength of the material. For the tests in this thesis the effect of decarburisation is thought to be small due to the relatively short term test durations. It must be remembered that decarburisation occurs continuously and the depths measured above are the depths at failure only. By the time the decarburised depth has reached significant levels, the bulk material is already heavily voided and also exhibits lower creep strengths. However, to be safe, tests of longer duration would probably be better performed on thicker gauge length specimens. The author is unaware of any work in the literature which has measured the creep strength of decarburised material.

\section{Summary of Uniaxial Creep Testing in Hydrogen}

- Creep lifetime is reduced in hydrogen compared with air. The effect is greatest at longer testing times.

- Minimum creep rate is higher in a hydrogen environment than in air.

- Elongation at failure and reduction of area are greatly decreased in hydrogen, with the effect being largest at longer testing times.

- Hydrogen attack cavitation was clearly observed in the specimens. The applied stress has greatly accelerated the damage processes (compare with section 5.1 where no cavities were seen without stress, and 5.2 where no cavities were seen in creep in air without hydrogen).

- Surface decarburisation retards cavitation. Under longer term testing conditions care must be taken to limit the size of the decarburised zone relative to the specimen dimension.

\begin{tabular}{|c|c|c|c|}
\hline Stress [MPa] & $\begin{array}{c}\text { Failure Time } \\
{[\text { [hours] }}\end{array}$ & $\begin{array}{c}\text { Strain to Failure } \\
{[\%]}\end{array}$ & $\begin{array}{c}\text { Reduction of Area } \\
{[\%]}\end{array}$ \\
\hline 104.9 & 212.9 & 44 & 63 \\
\hline 94.9 & 363.5 & 37 & 40 \\
\hline 86.8 & 503.2 & 21 & 26 \\
\hline 83.9 & 686.2 & 16 & 15 \\
\hline
\end{tabular}

Table 5.4: Summary of creep test data in hydrogen. 


\section{Hydrogen Pressurised Tubes}

Four tubes were pressurised with hydrogen gas at $600^{\circ} \mathrm{C}$ under internal pressures of 260, 240, 220 and 195 bar. These pressures correspond to mean diameter hoop stresses of 113.5, 104.7, 96.0 and 85.1 MPa respectively. The results show the first hoop strain creep curves from hydrogen pressurised tubes known to the author, and provide a detailed analysis of the damage which occurred in the tube wall.

\section{Comparison of Hvdrogen and Argon Pressurised Tubes Creep Curves}

Hoop strain vs time curves for the hydrogen pressurised tubes are shown in figure 5.34 alongside the creep curves obtained from argon pressurised tubes. As with the argon tests, only one tube was allowed to creep until it burst. The others were all interrupted at around 10\% strain. The creep rate is greater and the rupture time shorter for a given internal pressure in the hydrogen pressurised tubes. A pressure-rupture plot shows clearly the decrease in lifetime in figure 5.35, which confirms the results of tube burst testing found in the literature [Sticha'69], [Kolgat.' 59].

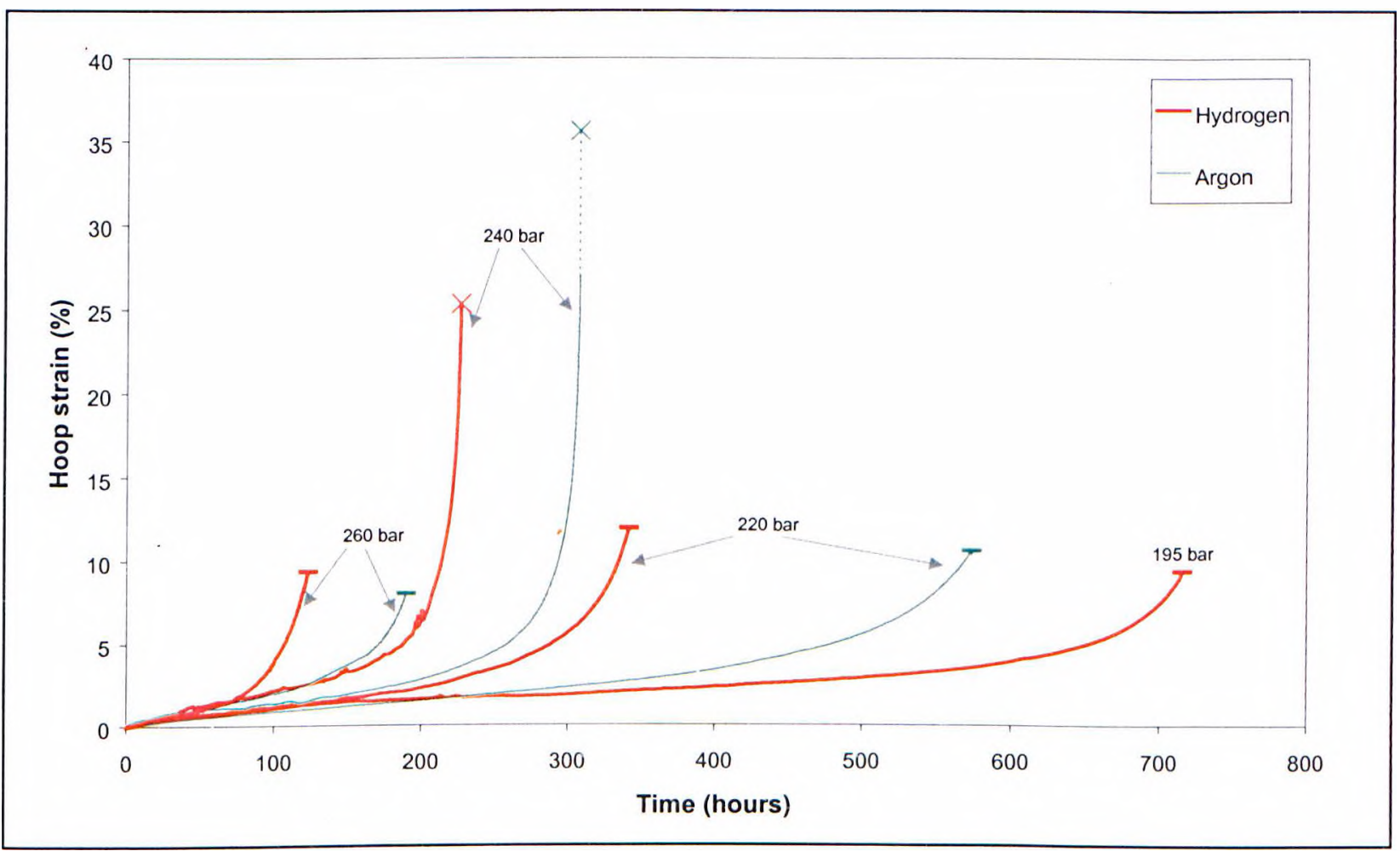

Figure 5.34: Hoop strain vs time creep curves for both hydrogen and argon pressurised tubes. The numbers represent the test pressures, and small horizontal lines at the end of the curves show that the test was interrupted prior to bursting. Note the decrease in rupture time for hydrogen pressurised tubes and increase in creep rate. 


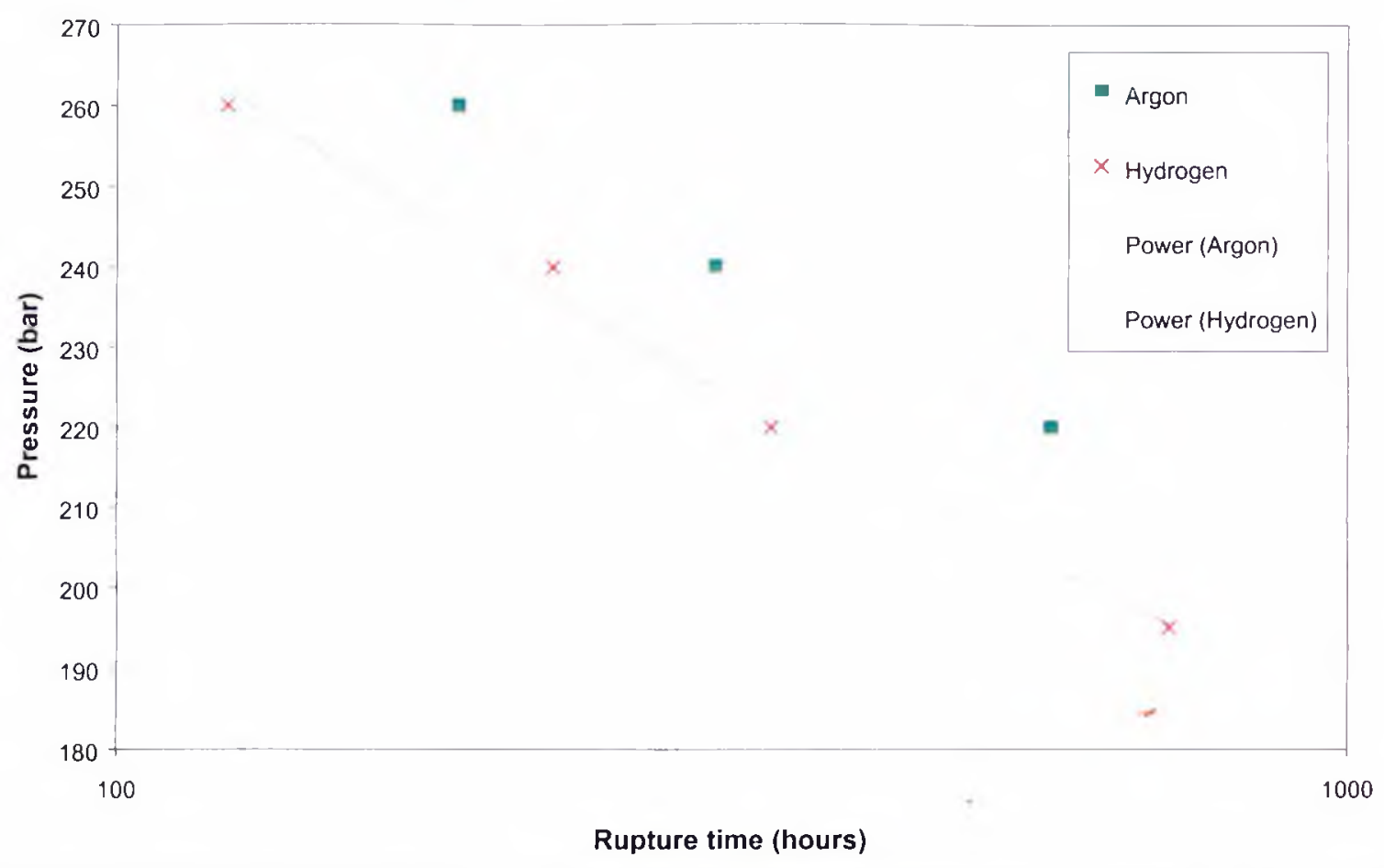

Figure 5.35: Pressure-rupture plots for hydrogen and argon pressurised tubes with power law curve fits. Note the clear reduction of lifetime when pressurising with hydrogen.

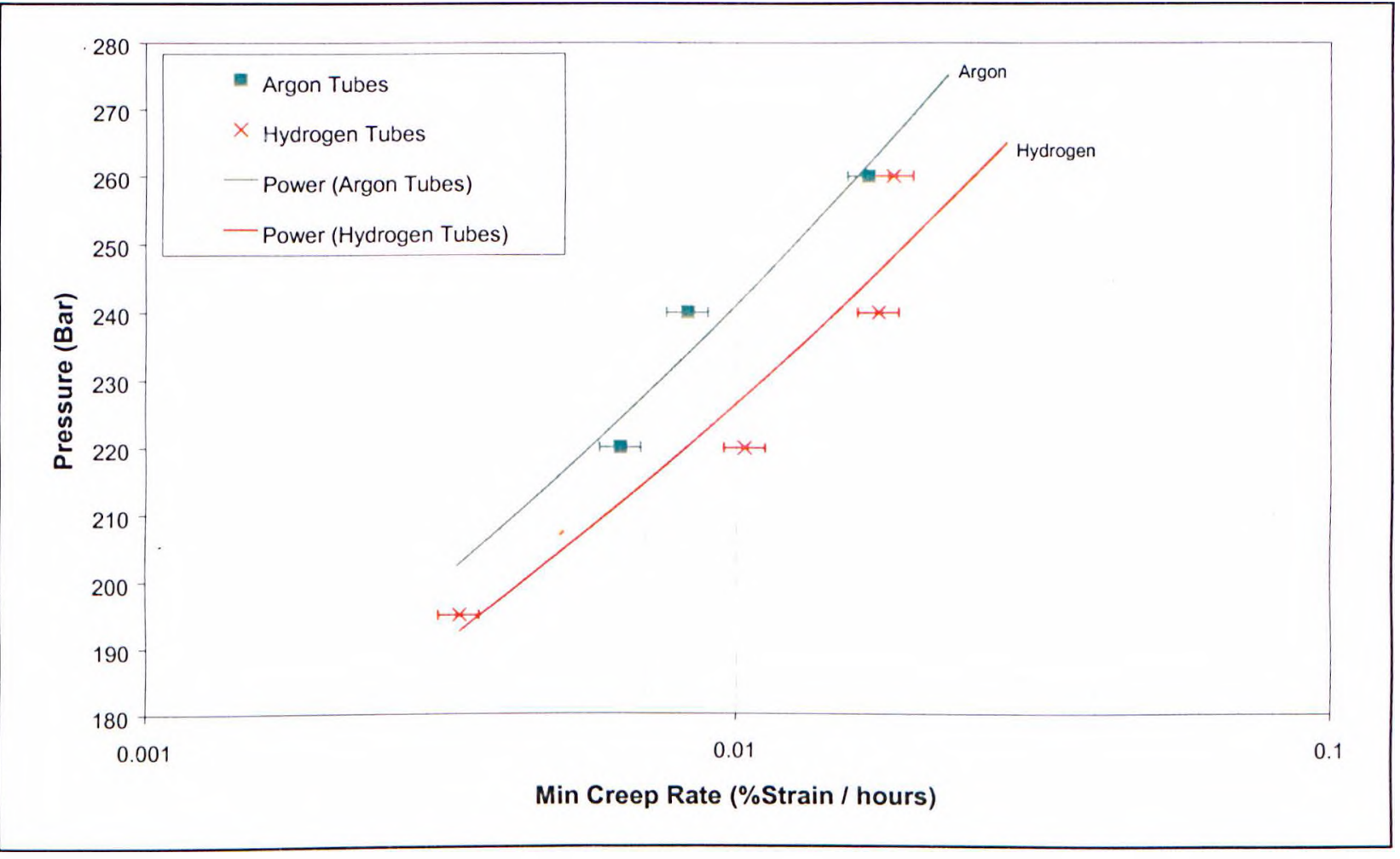

Figure 5.36: Minimum creep rate plot for tubes pressurised with hydrogen and argon. 
The slopes of the argon and hydrogen rupture curves are very similar, in contrast to the uniaxial rupture plot which showed a divergence at longer testing times. This could be caused by experimental scatter.

\section{Minimum Creep Rate}

Figure 5.36 shows the minimum creep rate plot for tubes pressurised with hydrogen and argon. Error bars represent the uncertainty in the manually measured creep rates. For a given internal pressure, the minimum creep rate is higher in hydrogen than argon. The slopes of the curves are similar ( $\mathrm{n}$ value in hydrogen is 7.54 , in argon 6.16), although there is some scatter in the data.

\section{Creep Ductility and Comparison with Argon Tests}

The creep curves show reasonably ductile behaviour within the conditions studied. Photographs

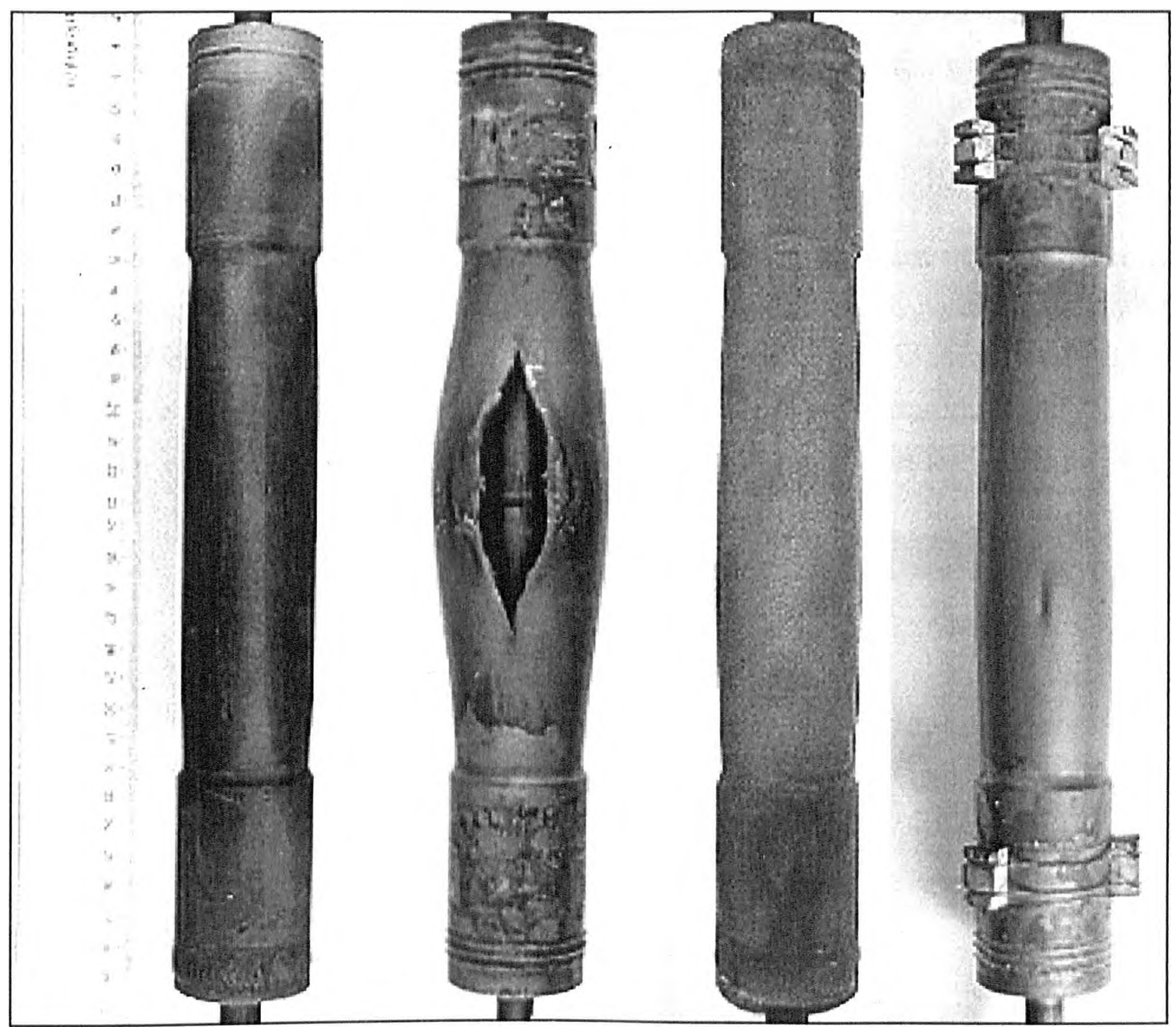

Figure 5.37: Photographs of the hydrogen pressurised tubes after testing. From left to right, pressurised with 260,240, 220 and 195 bars. Note the 195bar tube still has the ceramic spacers attached, and also shows a dip on the gauge length indicative of imminent failure 
of the tubes after testing are shown in figure 5.37. The tubes appear similar to the argon pressurised tubes (figure 5.13), except for the one tested at 195 bar which shows a depression on the gauge length, indicative of imminent failure.

The tubes burst with 240bar hydrogen and argon allow a direct comparison of failure ductility between the two pressurising mediums. The failure strain in hydrogen $(25.4 \%)$ is somewhat lower than the corresponding value in argon (35.6\%). The tube pressurised with 195bar hydrogen has a crack through virtually the entire wall thickness (figure 5.38). This tube was on the verge of bursting when the test was stopped, and thus gives an approximate strain to failure of $9.5 \%$. Analysis of the closest argon pressurised tube in terms of rupture time, being the one pressurised with $220 \mathrm{bar}$, shows no obvious damage at all at a similar strain of $10.5 \%$. However, the tube pressurised with 260 bar hydrogen also shows no serious damage at a strain of $9.4 \%$. It is therefore concluded that the effect of hydrogen pressurisation is to reduce the ductility (by an as yet undetermined amount), with the effect becoming greater under longer testing times. This trend is similar to that noticed for uniaxial creep specimens.

\section{Metallographic Damage Analysis}

The tube tested with 260 bar hydrogen, having a lifetime of only 123hours, showed little or no sign of hydrogen attack. Very few cavities were observed, and there was no easily discernible evidence of surface decarburisation. The microstructure was comparable to that of a tube tested in argon. Despite this the tube still failed much sooner than a tube pressurised with 260bar argon. This could be a result of experimental scatter, or it is conceivable that under the short

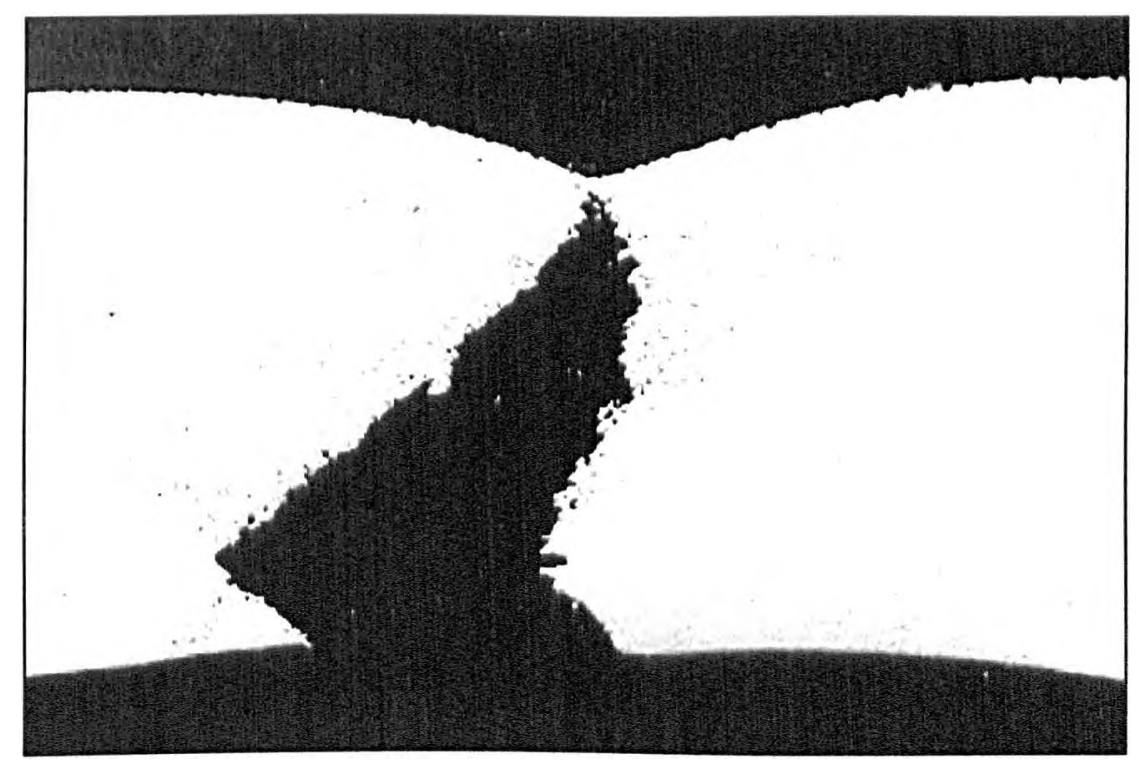

Figure 5.38: Transverse section of the tube pressurised with 195bar hydrogen, showing the through thickness crack (mag. x13). 
test duration a number of small cavities had grown that were too small to be observed. Theoretically, these could have had a weakening effect on the creep strength if their numbers were high enough. Alternatively, hydrogen itself may have an influence on the creep properties in the absence of any methane reaction. No evidence of this exists in the literature.

All of the other tubes showed cavitation near the inner surface. In general, very few cavities could be seen in the middle of the tube walls and none were observable near the outer surfaces. In effect, there was a gradient of cavitation observed, with cavitation being more severe near the inner wall. It was possible to define a rather subjective depth from the inner surface after which the size and number of cavities dropped off quite significantly. This depth was around $0.7-0.8 \mathrm{~mm}$ for all the tubes in which cavitation was observed, and is best illustrated for the 240 bar tube where the extra local strain near the rupture surface has caused the cavities to grow larger. This allows them to be seen under low magnification with the optical microscope (figure 5.39).

Cavities were generally noticed to be larger near the ends of cracks and in areas which had undergone large local strains. In other, less locally deformed areas, the cavity size, shape and distribution appeared to be similar between the 240, 220 and 195 bar tubes. Figure 5.40 compares typical SEM pictures taken from each of the above mentioned tubes at a position approximately $200 \mu \mathrm{m}$ from the inner surface. This position coincided with the position of maximum cavitation, since surface decarburisation tended to reduce the amount of cavitation

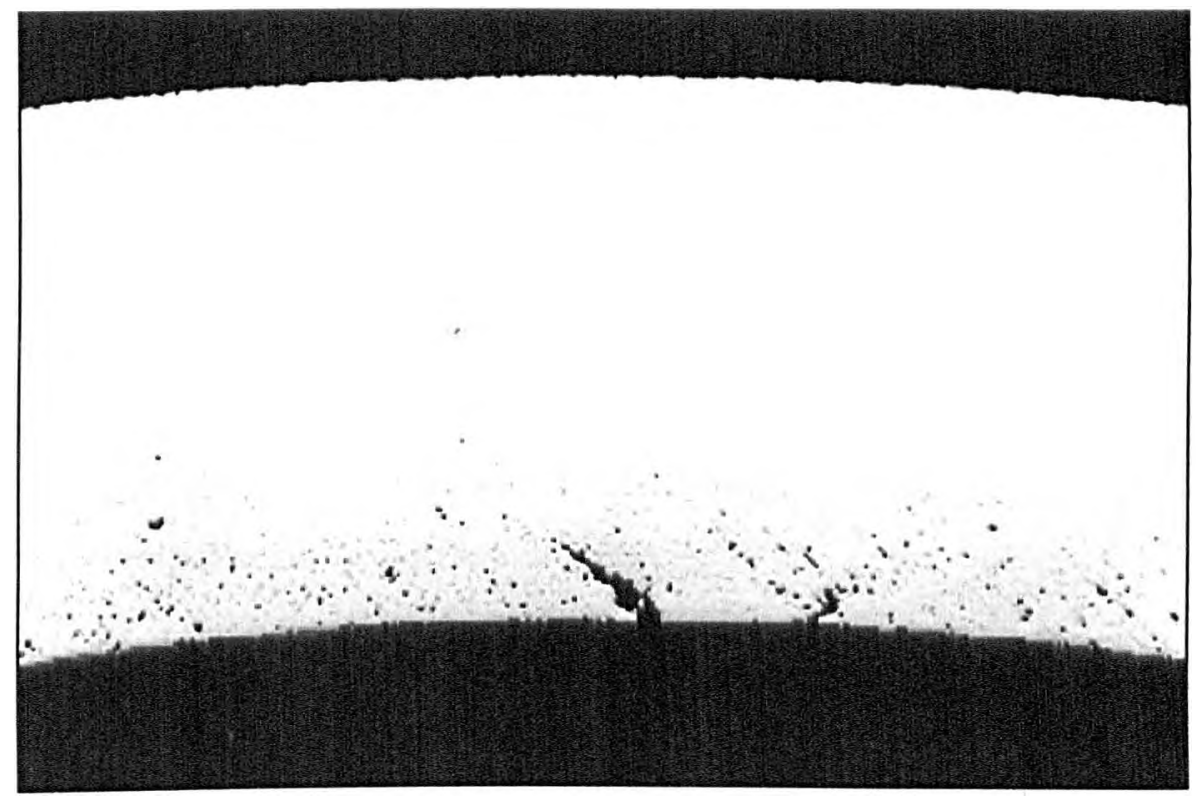

Figure 5.39: Transverse section of the tube pressurised with 240bar showing the cavity distribution and depth to which cavitation is significant. Note also coalescence of the cavities has lead to the formation of microcracks. (mag. xl6) 

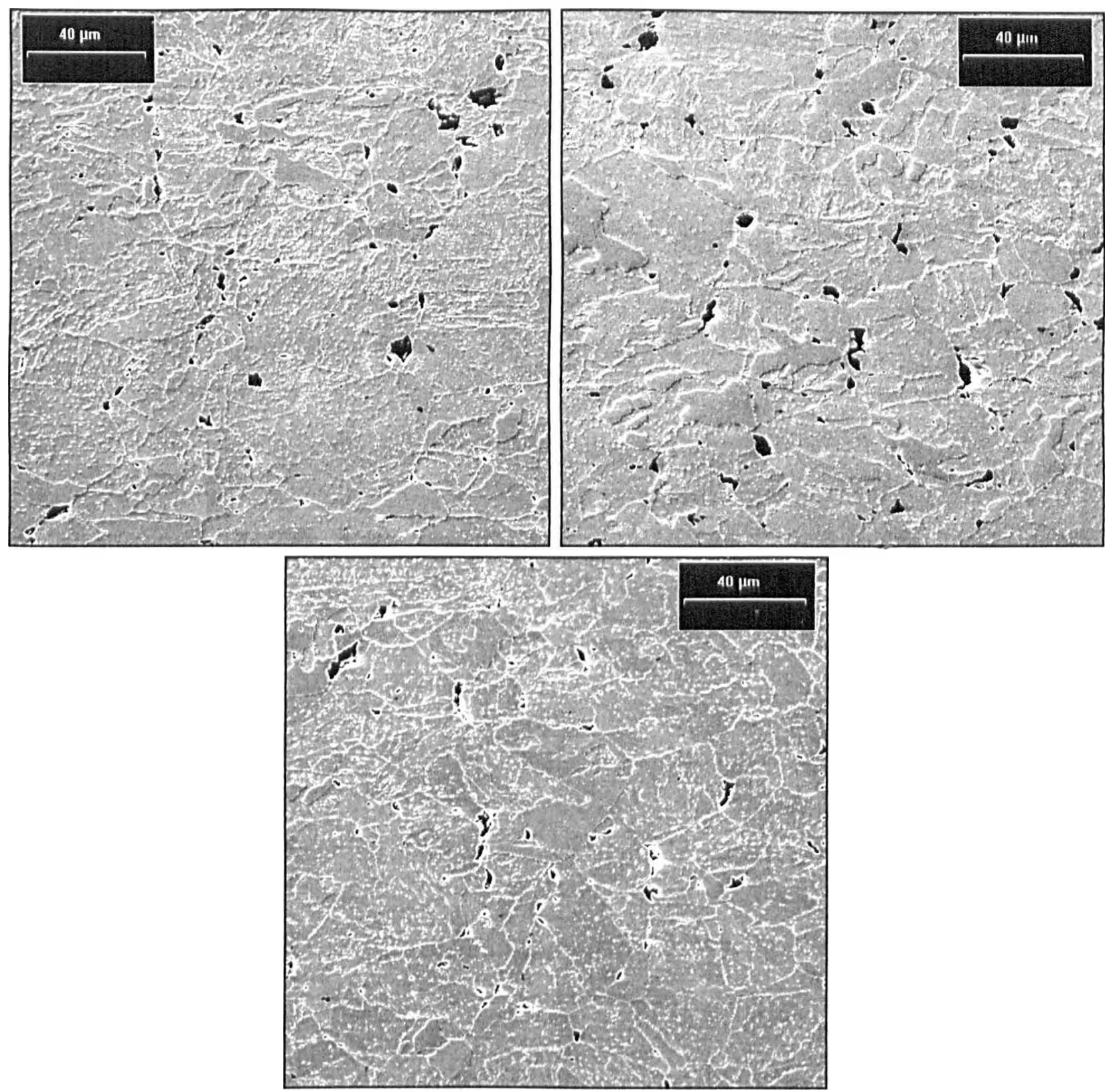

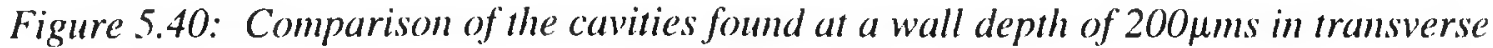
sections of the tubes pressurised with 240bar (upper left), 220bar (upper right) and 195bar (bottom) hydrogen. The non-uniform distribution makes finding trends difficult.

immediately next to the inner wall. The cavities range in size up to approximately $10 \mu \mathrm{m}$ with strings of cavities sometimes observed coalescing into larger voids. Cavity shapes are rounded, indicative of relatively fast surface diffusion [Chuang'79].

There is a tendency for coalescence of cavities to occur predominantly on those grain boundaries oriented at approximately $90^{\circ}$ to the hoop stress. Nevertheless, many other boundarics are cavitated regardless of their angle to the stress. The cavities are not uniformly scattered, with some grain boundaries having inter-cavity spacings of only 1 or 2 microns, whilst a large proportion of the boundaries are not cavitated at all. Spotting general trends in the 
cavitation between tubes was hampered by the non uniform cavity distribution, and the relatively small area in which cavities were seen. Given these difficulties, it is not easy to notice any significant differences in the observed microscopic cavity sizes and shapes between the 240, 220 and 195 bar tubes. This is in contrast to the observations for uniaxial creep, where a transition between the high and low stress tests was clearly noticed within a similar range of testing lifetimes.

The only clearly observed difference between the cavitation in the tubes was the increased tendency for the cavities in the 195bar tube to grow and coalesce on those grain boundaries oriented perpendicular to the applied stress. This alignment was best revealed in lower magnification photos taken with the optical microscope. For example, figure 5.41 compares optical micrographs of the tubes pressurised with 195bar and 220bar hydrogen (the 240bar pressurised tube looks much the same as the 220bar tube in terms of cavity alignment). This trend was also observed in the uniaxial specimens tested in hydrogen, although the trend there was more obvious.

Growth and coalescence of the cavities has led to the formation of microcracks which ultimately resulted in failure of the tubes. In the 240 bar tube, one crack propagated across the wall thickness, causing the tube to burst. Similarly, in the 195bar tube one crack had crossed about $95 \%$ of the wall thickness when the test was interrupted. These cracks will be referred to as "main" cracks in the following discussion. In all tubes except the 260bar tube, smaller cracks could be easily distinguished with the naked eye at the inner surface. Those small cracks which had attained a depth of around $0.5 \mathrm{~mm}$ or more will be referred to from now on as "secondary" cracks.
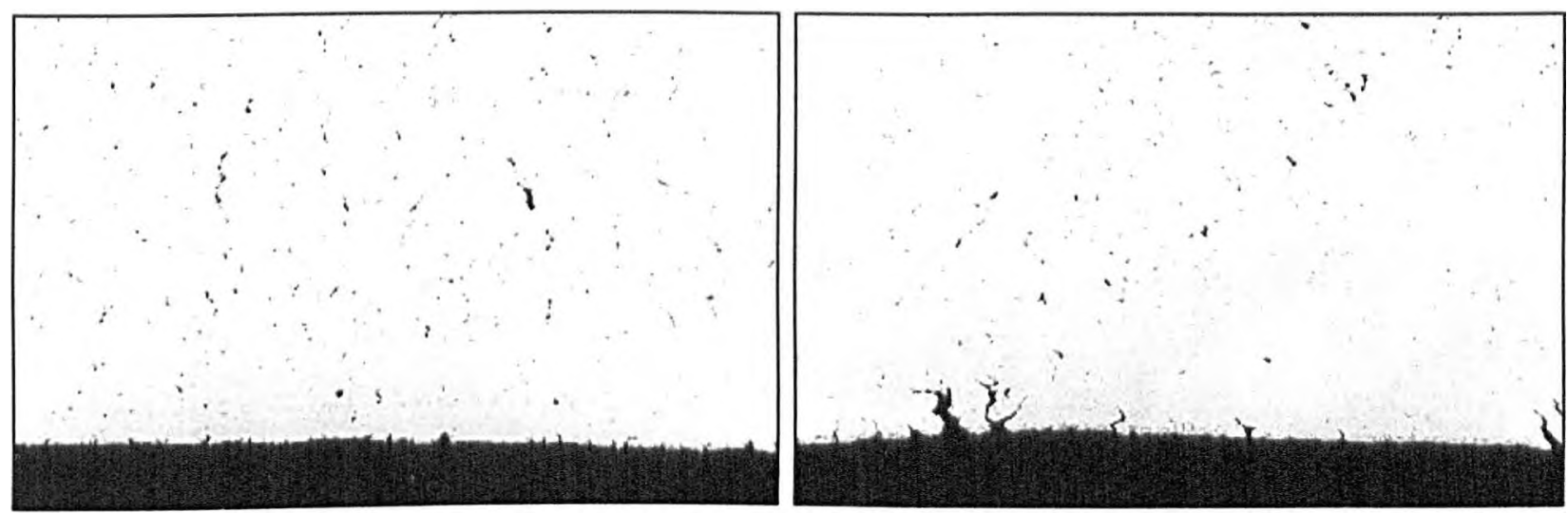

Figure 5.41: Comparison of optical micrographs showing transverse sections of the tubes pressurised with 19.5bar hydrogen for $71.5 \mathrm{hrs}$ (left) and 220bar hydrogen for 341hrs (right). Note the increased alignment of the cavities in the 195bar test perpendicular to the hoop stress. The hoop stress direction is horizontal. (mag. x11.5) 
In the tube pressurised with 240 bar hydrogen, seven such secondary cracks were observed when the tube was cut open for examination. The lengths of these cracks in the direction parallel to the gauge length ranged from about 1 to $8 \mathrm{~cm}$. The main crack had a length of some $10 \mathrm{~cm}$. In addition to the secondary cracks, there were also much finer, shallow surface cracks or depressions which were perceptible with the naked eye, often with similar lengths to the secondary cracks. These are referred to here as "valleys". The above features can be seen in figure 5.42. Closer examination of the cross section revealed that the secondary cracks had grown to depths of around 0.5-0.6 $\mathrm{mm}$ with an orientation of $45^{\circ}$ to the hoop stress direction Similarly, the main crack has a zigzag shape (figure 5.43).

The tube pressurised with 220bar hydrogen showed only two secondary cracks and no main crack. The secondary cracks nad a length parallel to the gauge of only $2 \mathrm{~mm}$ each. A small number of very fine "valleys" with lengths of a few mm's were also observed visually, although their small size made exact estimation of their lengths difficult. Closer examination of the cross section showed that the secondary cracks had depths of about $0.5 \mathrm{~mm}$ and were oriented at $90^{\circ}$

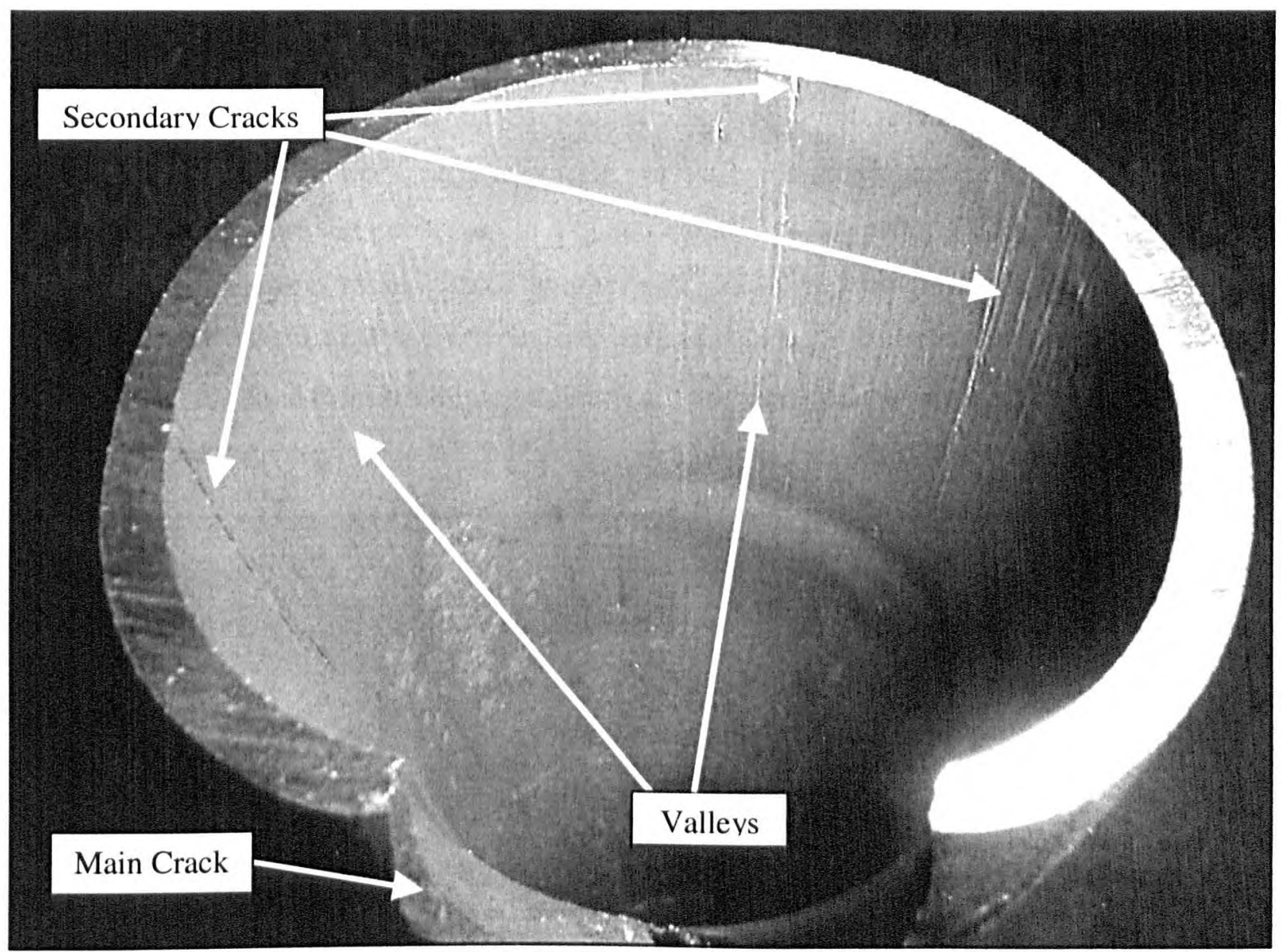

Figure 5.42: Photograph looking inside half of the tube burst with 240bar hydrogen. Note the presence of several secondary cracks, and smaller "valleys" 

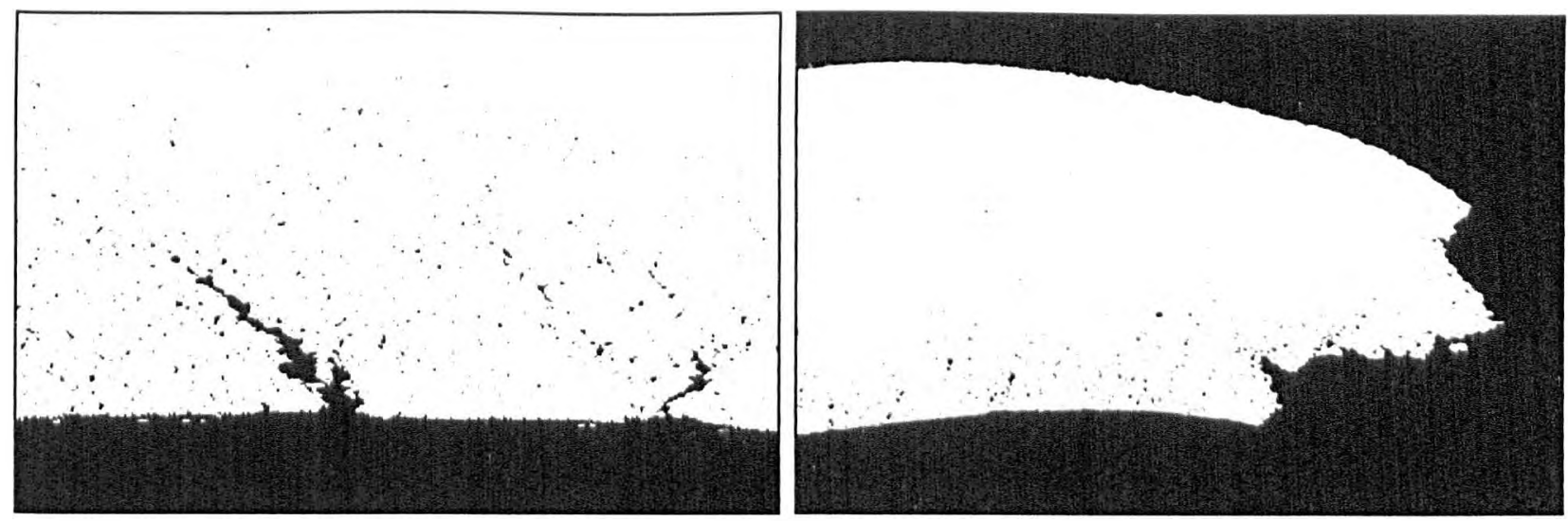

Figure 5.43: Preferential crack growth at 45" to the hoop stress in the tube pressurised with 240bar hydrogen. Left: typical secondary crack and 'valley' (mag. x35), and right: main crack with zigzag form (mag. x12)

to the hoop stress direction (figure 5.44). Cavitation was enhanced ahead of the crack tip.

Also apparent in the 220bar tube was the presence of a large number of very small surface cracks, each one having a depth of only about one or two grain facets. Some of these can be seen in figure 5.44. These microcracks were not observed in any of the other tubes tested. Similar style cracking was noticed in a study of the decarburised zone in unstressed samples [Masaok.'82], where it was suggested that the cracking occurred where the metal was decarburised to nearly zero carbon content, although no exact mechanism was determined. However, in the current work the cracks were not noticed in tubes pressurised with 260, 240 or 195 bars, neither were they observed in any autoclave exposed material. The secondary cracks probably initiated from these surface microcracks, although their presence does not seem to have greatly accelerated the secondary crack propagation in this tube. These microcracks could perhaps have initiated from machining defects, although no definite reason for their appearance has been found.
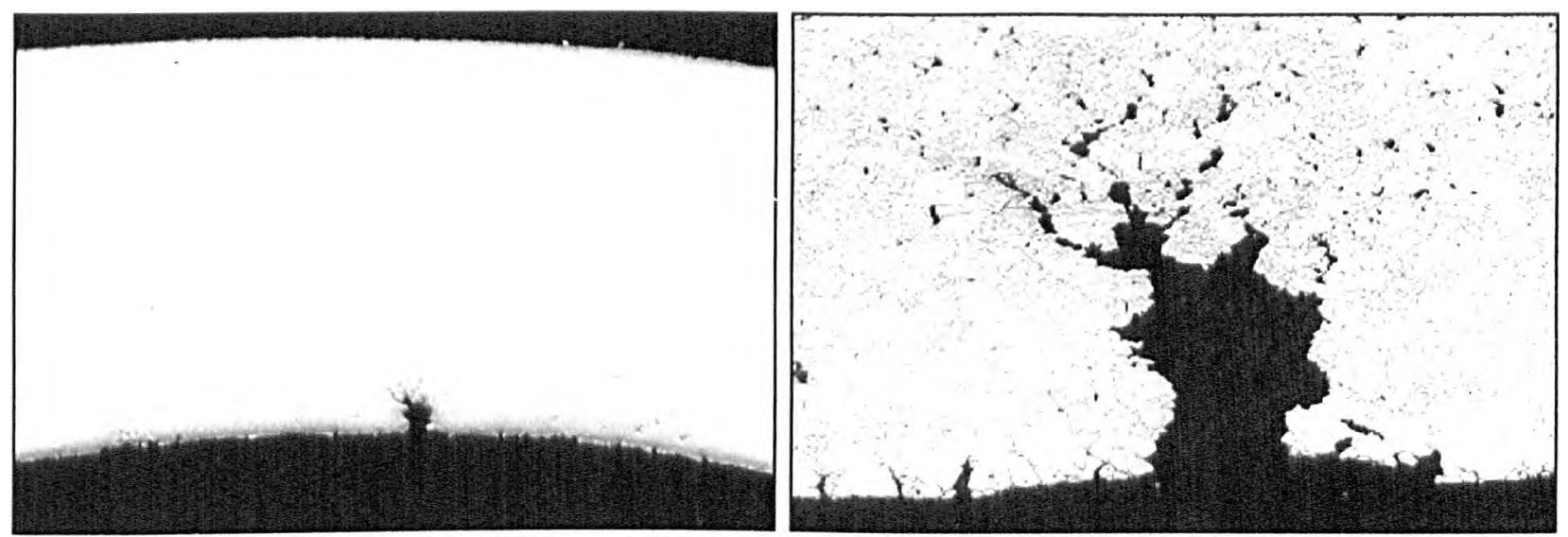

Figure 5.44: Secondary crack in the tube pressurised with 220bar hydrogen. Note the alignment at $90^{\circ}$ to the hoop stress and enhanced cavitation ahead of the crack tip. Left: mag. x12; right: $\operatorname{mag} x .80$ 
The tube pressurised with 195bar hydrogen showed a main crack with a zigzag shape as shown previously in figure 5.38. Two secondary cracks were also visible, having lengths parallel to the tube gauge of around $10 \mathrm{~mm}$ each, and depths of around $0.5 \mathrm{~mm}$. Further examination showed that these cracks were also growing at an angle of $45^{\circ}$ to the hoop stress, and showed projection of cavitation ahead of the crack tip. (figure 5.45).

There appeared to be some influence of the surface decarburised zone on crack initiation in the 195bar tube. For example, figure 5.45. shows clearly the decarburised surface as a lighter band. The crack faces in this band are relatively smooth compared with the more ragged intergranular appearance of the rest of the crack. Detailed examination of the tube revealed other features which were not obvious in the other tubes. Figure 5.46a shows what appears as a valley. on the inner surface to be a rather significant secondary crack within the material, with relatively ductile thinning occurring at the surface. Figure5.46b shows how a crack can initiate inside the material, and not from the inner surface. These findings can be explained in terms of the surface decarburised layer. Cavitation in this layer is reduced due to the lack of carbon. In addition, the expected lower strength of the decarburised layer means that stress redistribution in the tube wall probably results in a lower stress being supported by the decarburised zone. Both of these factors reduce the chance for cavity growth and coalescence to microcracks. Thus, cavitation is greater just beyond the decarburised zone so this is where the microcracks initiate. Growth of

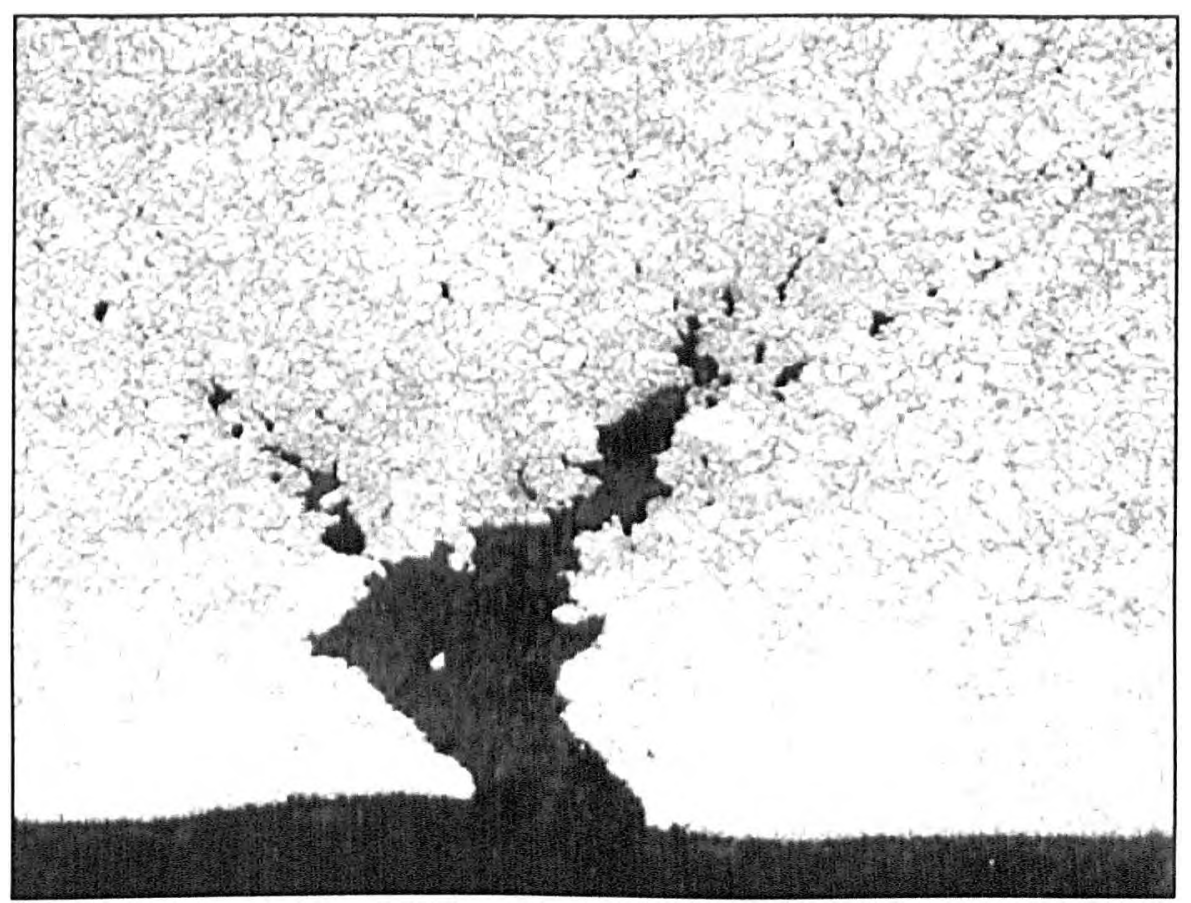

Figure 5.45: Secondary crack in the tube pressurised with 195bar hydrogen. Note the $45^{\circ}$ alignment to the hoop stress, projection of cavities ahead of the crack, and the surface decarburised zone (etched $5 \%$ Nital, mag. x100) 


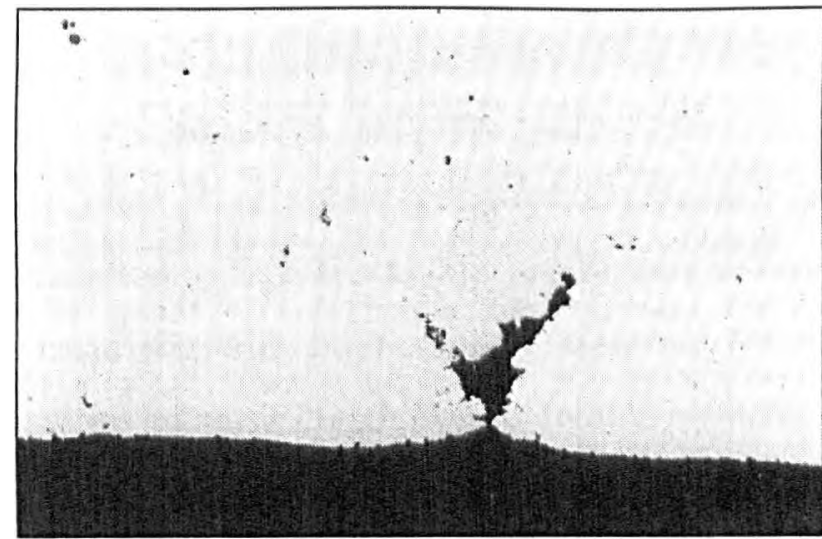

a)

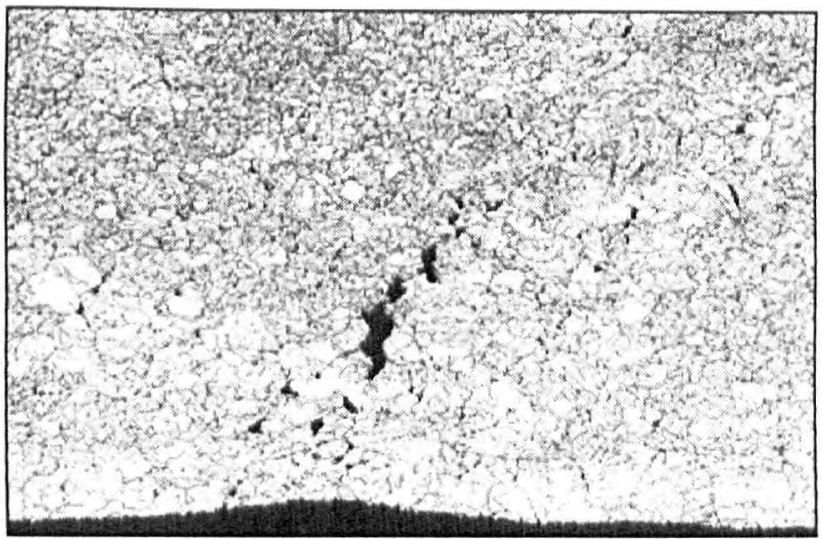

b)

Figure 5.46: The effect of surface decarburisation on crack initiation in the 195har tube. a) Appears as a valley on the swrace but has significant depth below the surface. Also shows relatively ductile thinning in the decarburised surface zone. (mag. x60); b) Crack initiation away from the surface, just beyond the decarburised zone (etched 5\% Nital. mag. x75)

the crack to the inner surface may then take place in a relatively ductile fashion, by shearing (figure 5.45) or by ductile thinning of the decarburised layer (figure 5.46a). This phenomenon was not obvious in the other tubes probably because the decarburised zone was not so large, due mainly to the shorter testing times involved. It is therefore expected that, under even longer testing conditions, the phenomenon could become yet more important. If this form of crack initiation is important under industrial conditions, then it may have some influence on the choice of NDE method to detect hydrogen attack damage, due to its sub-surface nature.

\section{Discussion of Damage Evolution in Hvdrogen Pressurised Tubes}

It is clear that, unlike tubes pressurised with argon, hydrogen pressurised tubes undergo serious damage which lowers their lifetime and ductility. This damage is typical of hydrogen attack; being surface decarburisation and grain boundary cavitation which leads eventually to cracking. The following discussion aims to bring together many of the points made under the previous heading of "Metallographic Damage Analysis" by proposing mechanisms for the attack evolution.

It is assumed that, on initial pressurisation, hydrogen gas quickly dissociates and enters the steel. A hydrogen concentration gradient is assumed to build up rapidly due to the fast diffusion rate of hydrogen. The concentration is high near the inside and low, or possibly zero on the outside of the tube. This hydrogen which has entered the steel can begin to react with carbon to form methane, and hence create hydrogen attack cavities by the normally accepted mechanisms. A cavitation gradient is observed which reflects the hydrogen concentration gradient across the 
tube wall. Hydrogen concentration is therefore important, yet the tubes pressurised with different pressures (different concentrations) all show similar depths to which cavitation is apparent. Those tubes pressurised with high hydrogen pressures would be expected to show cavitation to a greater depth than those pressurised with lower pressures. However, those tubes pressurised with high-pressure also had the shortest failure times which did not allow the cavities to grow much before failure occurred. Conversely, at the lower pressures the lower hydrogen concentration at a given depth slowed down cavity growth rates, but the longer test durations gave the cavities time to be seen to the same depth as the high-pressure tests.

At the same time, the methane reaction is occurring on the steel surface, depleting the carbon supply and creating a decarburised zone. The decarburised zone probably has lower strength due to the lack of carbides, and may therefore support less stress due to stress redistribution which can occur within the tube wall. Cavitation in the decarburised zone is generally restricted because of the low carbon content and reduced stress. It has been reported in the literature that surface decarburisation and cavitation are competing processes, with the amount of cavitation observed being dependent on the relative kinetics of the two processes [Rosent.'63]. Thus, a distribution of cavitation is observed in the tubes, with lower cavitation to a depth approximating that of the decarburised zone, followed by a maximum of cavitation just beyond the decarburised layer and a gradual depletion towards the outer wall. The effect of decarburisation on cavitation was best noticed in the tube pressurised with 195bars hydrogen, where the decarburised layer was deeper than in the other tubes (due to the longer time at temperature).

Cavity growth and coalescence leads to the formation of microcracks. Coalescence occurs preferentially on those grain boundaries oriented at $90^{\circ}$ to the hoop stress which highlights the importance of the MPS in controlling cavitation. The tendency for coalescence on $90^{\circ}$ boundaries is increased slightly under longer testing times. The initiation location of these cracks depends on the relative kinetics of cavity growth and surface decarburisation. In the tubes pressurised with 240 and 220 bar hydrogen, the cracks appear to initiate at or very near to the inner surface. However, because of the relatively fast decarburisation, the tube pressurised with 195bar initiated cracks at a short distance from the surface. Crack initiation seems to occur at lower macroscopic strains for longer term tests. This is in parallel to the increased loss of ductility with longer test times observed for uniaxial creep testing in hydrogen. The $240 \mathrm{bar}$ tube showed more secondary cracks than the others. One explanation for this is the burst type failure which may have opened several of the smaller valleys into significant secondary cracks. 
Once initiated, the cracks appear to grow relatively quickly in the direction parallel to the tube gauge length. This is because the material in this direction is already damaged with cavitation and provides an easy crack path. Similarly, the cracks also seem to be able to grow easily to a depth of about $0.5-0.6 \mathrm{~mm}$ which corresponds to the depth at which cavitation is most apparent. Once a secondary crack is open to the inner surface, it allows the surface conditions to be present at the crack tip, i.e. the hydrogen concentration at the crack tip is increased. In addition, a stress concentration will build up at the crack tip and, together with the increased hydrogen concentration, this causes cavities to be nucleated and grow in a region ahead of the crack tip, often in a string aligned at $45^{\circ}$ to the hoop stress (figure 5.45). Local cavity growth of this type is one of the reasons why the cavity distribution was so inhomogeneous. Initially, the cracks are aligned at $90^{\circ}$ to the hoop stress (figure 5.44). However it appears that once the crack length in the parallel direction to the gauge length becomes greater than a few mm's, then the crack orientation becomes $45^{\circ}$. This can be deduced by comparing the $90^{\circ}$ cracks in the $220 \mathrm{bar}$ tube and the $45^{\circ}$ cracks in the 240 and 195 bar tubes. The only noticed difference between the cracks was the shorter length in the gauge direction' for the 220bar tube. The crack length was short because the test was interrupted before failure, whereas the 240bar tube had burst, and the 195bar tube was on the verge of bursting when interrupted. It is proposed that the increased length of the cracks augments the stress concentration found around the crack. This stress concentration may then be responsible for driving the crack at a $45^{\circ}$ angle, and demonstrates a change in controlling stress from MPS to shear.

Once one of the secondary cracks attains a depth greater than $0.6 \mathrm{~mm}$ or so, it propagates rapidly across the wall and causes failure. This can be deduced because none of the secondary cracks were deeper than $0.6 \mathrm{~mm}$. It is suspected that the high stress concentration ahead of such cracks, and the cavitation projected ahead of them cause the main crack to grow very rapidly, so that ductile failure mechanisms take over once the crack is more than about one third of the way through the wall.

\section{Summary of Hydrogen Pressurised Tube Testing}

- Tubes pressurised with hydrogen exhibit shorter lifetimes and higher creep rates than tubes pressurised with argon.

- Tube ductility is reduced in hydrogen.

- Failure occurs by cracking, initiated from the growth to coalescence of cavities. 
- Initial cavity initiation/growth is controlled by the maximum principal stress in the tube. This is most apparent in longer term tests.

- Cavitation is predominant in all of the tubes to a depth of around $0.75 \mathrm{~mm}$ or about $17 \%$ of the original wall thickness.

- Cavity coalescence leads to the formation of microcracks, which shortly after initiation become oriented at $45^{\circ}$ to the maximum principal stress due to the stress concentrations which surround them.

- Cavitation is enhanced around crack tips due to the stress concentration and the increased hydrogen concentration at the crack tip.

- Secondary cracks grow easily to a depth of about $0.6 \mathrm{~mm}$ through the heavily cavitated area. Cracks larger than this seem to grow very quickly across the tube wall to cause failure.

- Surface decarburisation under long testing times can hinder cavity formation near the surface, thus changing the crack initiation process.

\begin{tabular}{|c|c|c|c|}
\hline Pressure [bar] & $\begin{array}{c}\text { Mean Diameter } \\
\text { Hoop Stress [MPa] }\end{array}$ & $\begin{array}{c}\text { Rupture Time } \\
\text { [hours] }\end{array}$ & $\begin{array}{c}\text { Failure Hoop } \\
\text { Strain [\%] }\end{array}$ \\
\hline 260 & 113.5 & $123^{*}$ & $9.4^{*}$ \\
\hline 240 & 104.7 & 226 & 25.4 \\
\hline 220 & 96.0 & $342^{*}$ & $12^{*}$ \\
\hline 195 & 85.1 & $715^{*}$ & $9.5^{*}$ \\
\hline
\end{tabular}

* Interrupted test

Table 5.5: Summary of hydrogen pressurised tube test data.

\section{Comparison Between Uniaxial and Tube Tests in Hydrogen}

\section{Damage Observations}

Several differences in the damage evolution between uniaxial specimens and pressurised tubes have already been mentioned. The evolution with decreasing stress from well spaced, homogenous cavities to small, closely spaced cavities on boundaries at $90^{\circ}$ to the stress, which was clearly seen in uniaxial specimens was not so obvious in the tube tests, despite similar testing lifetimes. Comparisons of the microstructures reveal that, in general, the cavities appear more numerous and larger in uniaxial specimens for similar test lifetimes (figure 5.47). However, variation in the testing conditions (table 5.6) mean that it is difficult to explain the cavity size differences in terms of stress or hydrogen pressure which are interrelated in the 
pressurised tubes. Also apparent in figure 5.47 is the increased homogeneity of cavitation in the uniaxial specimens when compared with tube tests.

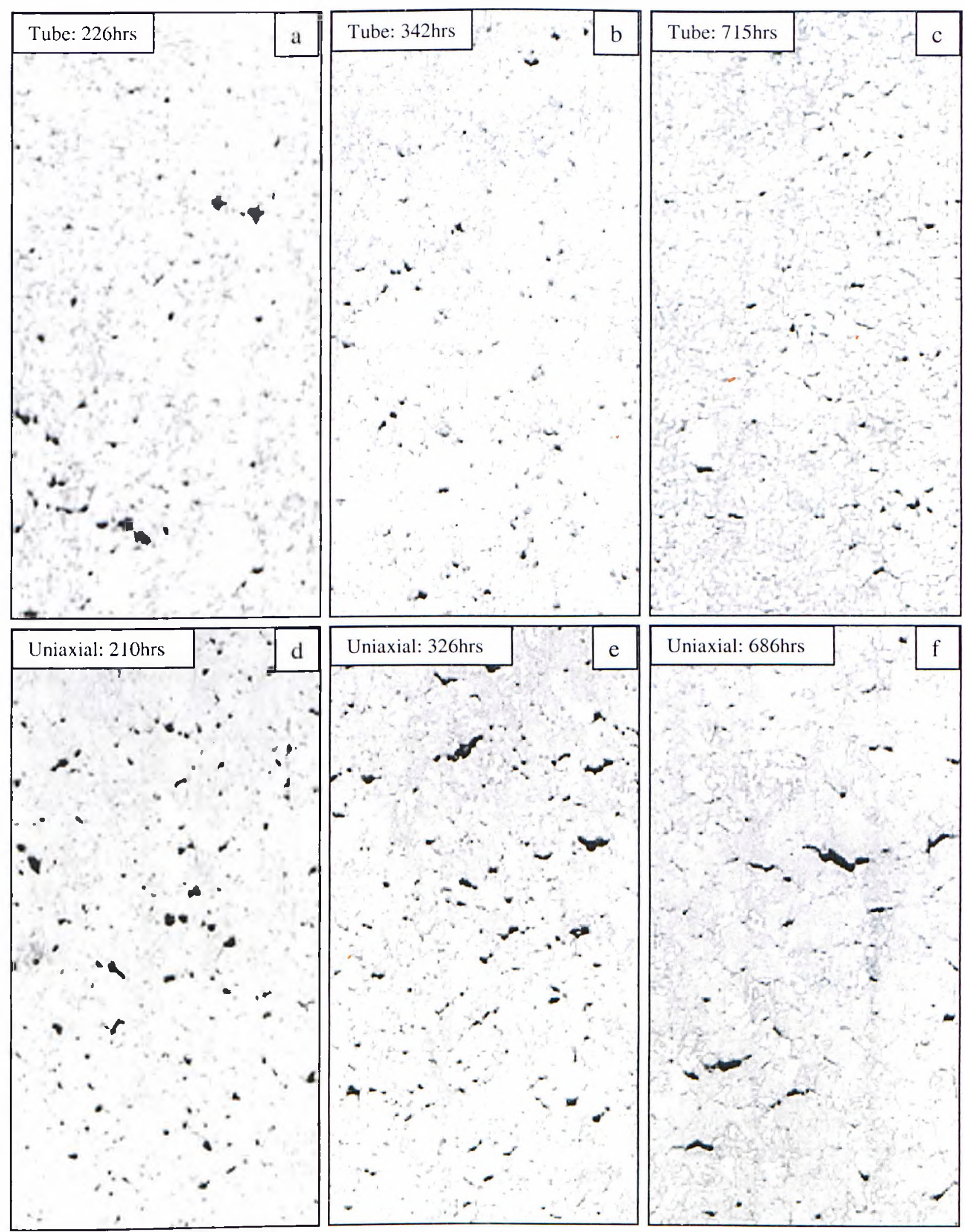

Figure 5.47: Comparison of cavitation in tubular(a-c) and uniaxial (d-f) specimens for similar testing times. The principal stress direction is vertical. Note the increased homogeneity, size and number of cavities in the uniaxial specimens for a given testing time. (All specimens etched in 5\% Nital, mag. x100) $200 \mu \mathrm{m}$ 


\begin{tabular}{|c|c|c|c|c|c|c|c|}
\hline \multicolumn{4}{|c|}{ Uniaxial } & \multicolumn{5}{c|}{ Tulses } \\
\hline $\begin{array}{c}\text { Hydrogen } \\
\text { Pressure } \\
{[\text { Bar] }}\end{array}$ & $\begin{array}{c}\text { Initial } \\
\text { Stress } \\
{[\mathrm{MPa}]}\end{array}$ & $\begin{array}{c}\text { Rupture } \\
\text { Time } \\
\text { [hours] }\end{array}$ & $\begin{array}{c}\text { Failure } \\
\text { Strain [\%] }\end{array}$ & $\begin{array}{c}\text { Hydrogen } \\
\text { Pressure } \\
{[\text { Bar] }}\end{array}$ & $\begin{array}{c}\text { Initial } \\
\text { Mean } \\
\text { Diameter } \\
\text { hoop stress } \\
{[\mathrm{MPa}]}\end{array}$ & $\begin{array}{c}\text { Rupture } \\
\text { Time } \\
{[\text { hours] }}\end{array}$ & $\begin{array}{c}\text { Failure or } \\
\text { Interrupted } \\
\text { Hoop } \\
\text { Strain [\%] }\end{array}$ \\
\hline- & - & - & - & 260 & 113.5 & 123 & 9.4 int \\
\hline 220 & 104.9 & 210 & 44 & 240 & 104.7 & 226 & 25.4 \\
\hline 220 & 94.9 & 326 & 37 & 220 & 96.0 & 342 & 12.0 int \\
\hline 220 & 86.8 & 503 & 21 & - & - & - & - \\
\hline 220 & 83.9 & 686 & 16 & 195 & 85.1 & 715 & 9.5 int \\
\hline
\end{tabular}

Table 5.6: Comparison of testing conditions for the uniaxial and tubular specimens tested with a hydrogen environment. Those tests with similar rupture times are shown highlighted in the table. Since stress increases with strain, only the initial stress is included in the table.

Mean diameter hoop stress is used for the tubes because of its simplicity and success in correlating rupture behaviour in tubes pressurised with argon.

For a given test time, the level of damage in the tubes appears lower than in uniaxial specimens. This would suggest that the 'damage' tolerance of the tubes is lower than for uniaxial specimens. However, figure 5.47 shows only the general.damage situation in the tubes, whereas the presence of secondary cracks clearly indicates that the damage may be locally much higher in the tubes. For example, figure 5.48 shows the cavitation damage observed in a small area of the tube pressurised with 195bar hydrogen. This shows much greater cavitation than figure $5.47 \mathrm{c}$, and shows similar, if not greater, cavitation than in figure 5.47f. Thus, in small, localised

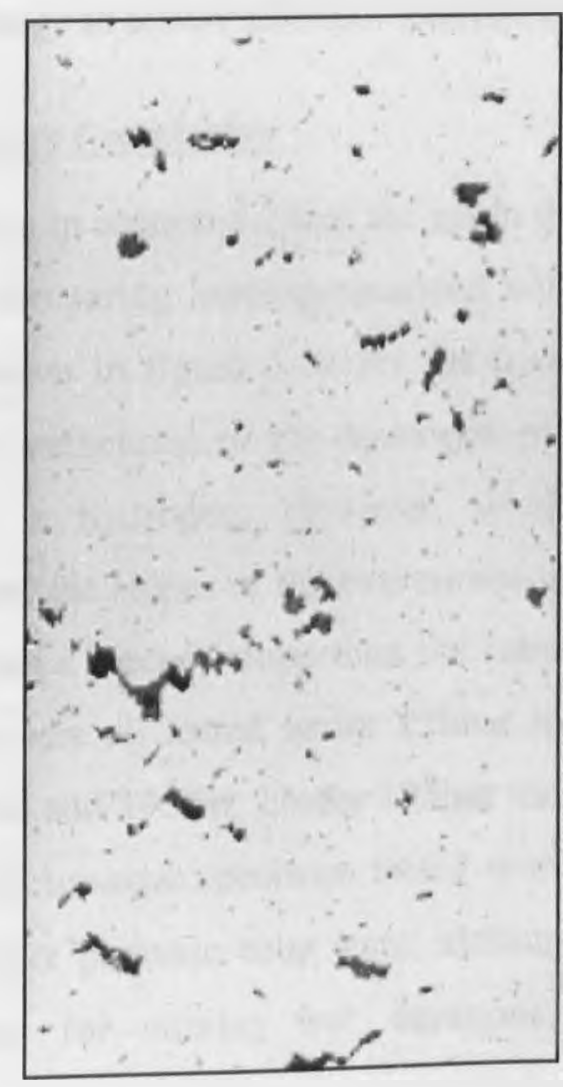

Figure 5.48: Locally enhanced cavitation in the tube pressurised with 195bar hydrogen (715hrs). Compare with figure $5.47 \mathrm{c}$ and 5.47f. The principal stress direction is vertical (mag. $x 100$ ) 
areas of the tubes, the cavity damage situation is similar to or worse than the uniaxial case. Locally enhanced cavitation of this sort is responsible for initiating the secondary cracks which lead ultimately to failure of the tube and is one of the main differences in the behaviour of uniaxial and tubular specimens. Uniaxial specimens exhibit uniform damage throughout the whole of the gauge thickness and length (with the exception of the decarburised zone and the region immediately next to the fracture). Tubes however are able to support local damage which may be higher than the average damage in the tube.

The lower numbers and sizes of cavities seen elsewhere in comparison with the uniaxial situation may be a result of stress redistribution. As the inside of the tube becomes damaged, it wants to strain faster for a given applied stress. However, the relatively healthy material towards the outside of the tube wall restrains the inside. The result is that the stress on the inside of the tube will drop, thereby lowering the driving force for cavity nucleation and growth. Similar stress redistribution may also allow the tube to exhibit the non uniform cavitation.

To summarise, the main difference between uniaxial and tubular specimens is the distribution of damage with uniaxial specimens having much more uniform damage distributions. Similarities exist between the damage at failure in uniaxial specimens and the local damage near secondary cracks in tubes. Thus, for similar test times and stresses, there appears to be a critical damage to failure for both uniaxial and tube tests.

\section{Creep Rupture Correlation}

It was shown in section 5.2 that the mean diameter hoop stress provided a reasonable reference stress for comparing tubes pressurised with argon and uniaxial specimens tested in air. The same is shown in figure 5.49 for the hydrogen tests. The mean diameter hoop stress gives reasonable predictions of the hydrogen pressurised tube behaviour from uniaxial creep tests performed in hydrogen. However, when comparing the rupture behaviour in an inert environment, the slopes of the two curves were approximately equal. Here the uniaxial creep in hydrogen has a steeper slope than the tubes. A possible explanation is that the uniaxial creep specimens were all tested under 220 bar hydrogen. The tubes on the other hand were tested between 260 and 195bar. Under 195bar the tube may have experienced lower hydrogen attack kinetics than uniaxial specimen tested with 220 bar hydrogen. The opposite could also be true for the higher pressure tube tests, although the previous discussion quoted similar damage observations for similar test durations, independent of the test pressure conditions. 
Alternatively, the different behaviour may be due to experimental scatter. More detailed analysis and predictions of the rupture time will be made in section 6 of the thesis.

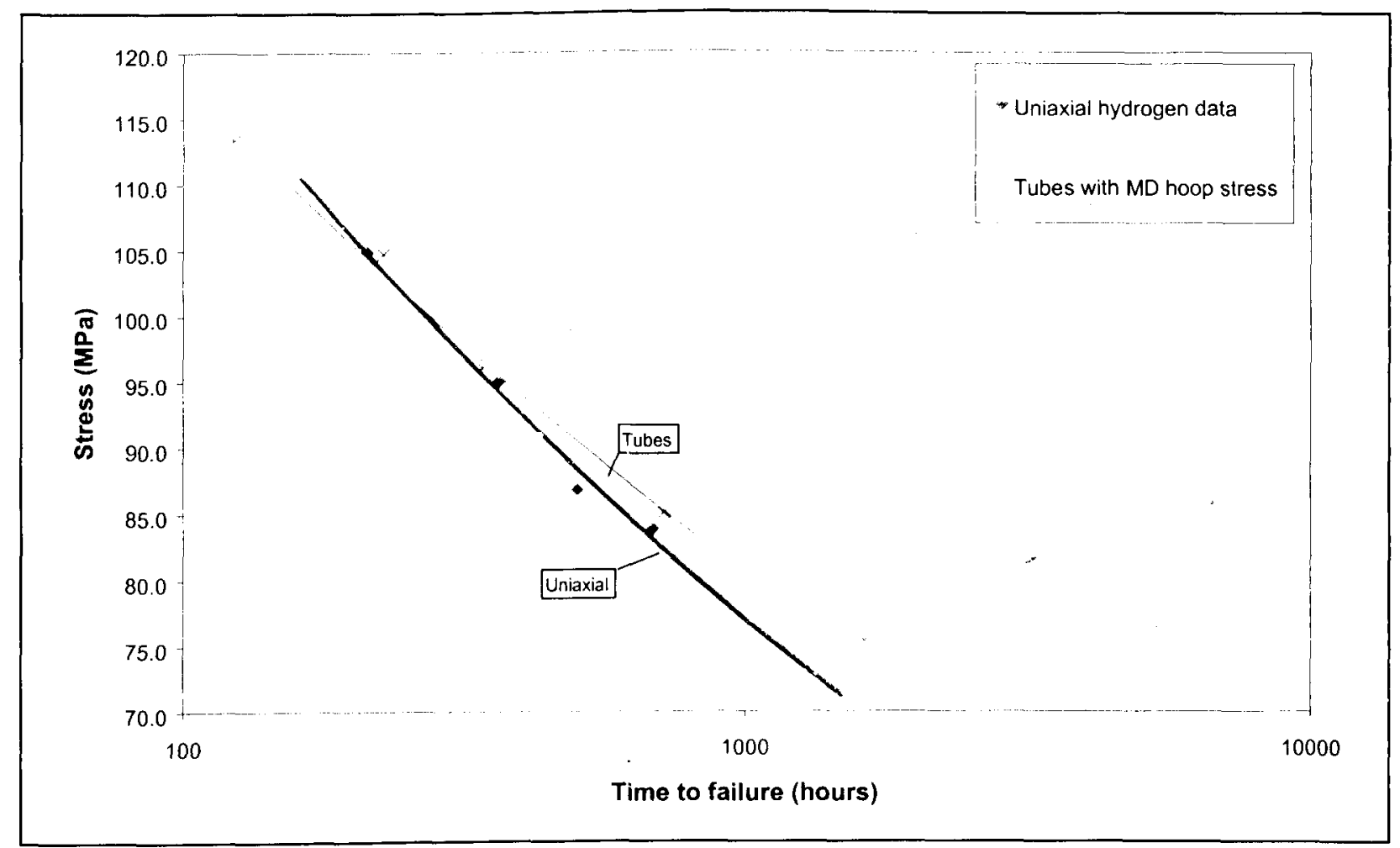

Figure 5.49: Correlation of uniaxial and hydrogen pressurised tubes rupture times using the mean diameter hoop stress.

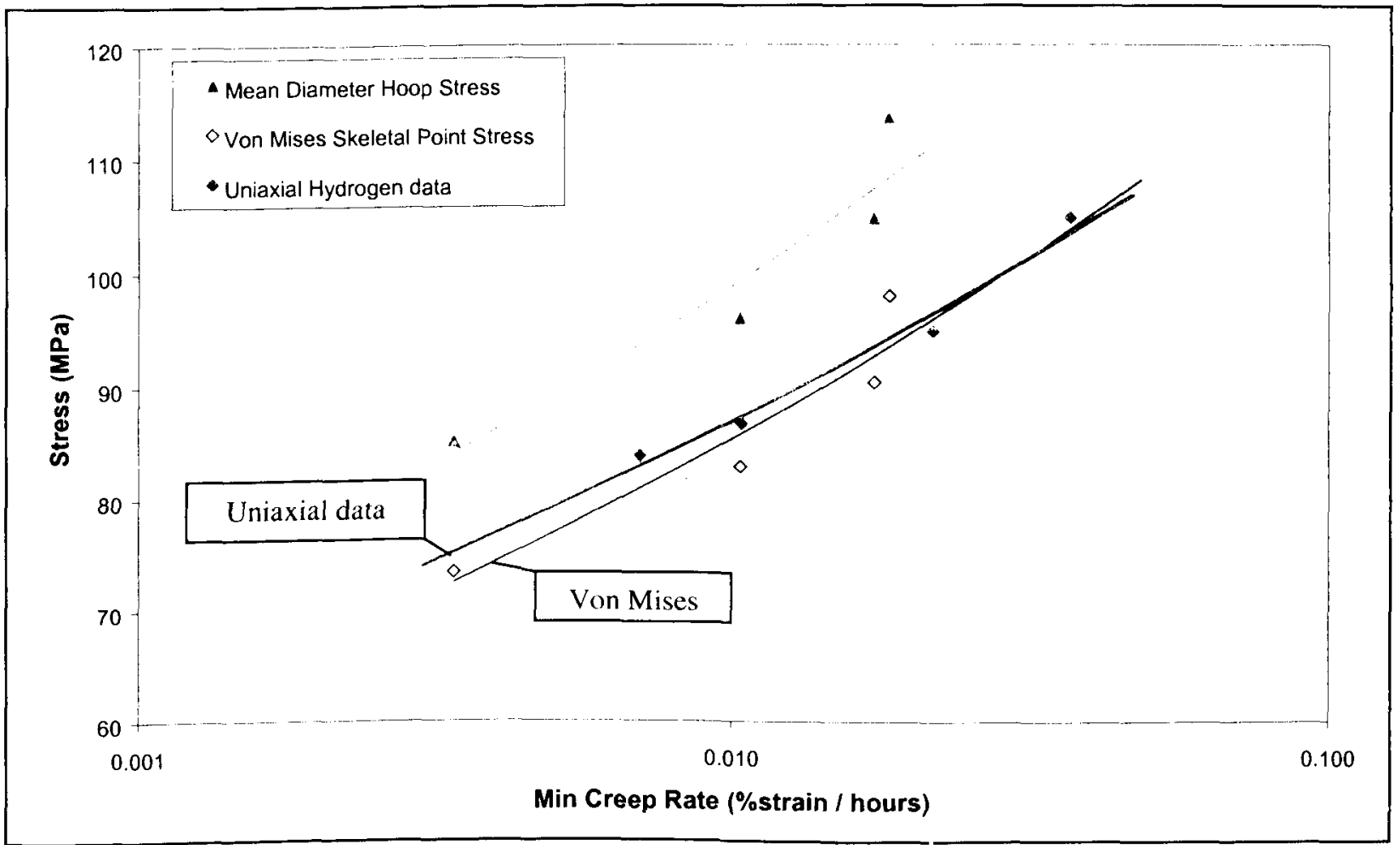

Figure 5.50: Correlation of minimum principal strain creep rate from hydrogen pressurised tubes and uniaxial minimum creep rate in hydrogen using the mean diameter hoop stress and the thick wall Von Mises skeletal point stress. 


\section{Minimum Creep Rate Correlation}

Figure 5.50 shows the correlation of the minimum creep rate for uniaxial creep specimens tested in hydrogen and tubes pressurised with hydrogen. Similar to the argon/air correlation, the skeletal point Von Mises stress is shown to give a good reference. 


\section{CREEP CURVE MODELLING}

The modelling approach used here is based around fitting uniaxial creep curves with a curve fitting equation, then predicting the creep behaviour of internally pressurised tubes from these uniaxial curve fits. The internally pressurised tube tests presented in section 5.3 act as benchmark verification tests for the predictions. Further development of the model could be applied to plant components.

Initially, the creep curve fitting routines will be discussed in relation to uniaxial creep data in air and hydrogen environments. The model parameters determined for uniaxial creep in air will then be used to predict the hoop strain creep curves of argon pressurised tubes by making certain assumptions about the material behaviour under multiaxial stresses. A more complex method will then be used to predict the creep behaviour of hydrogen pressurised tubes by using a concentric cylinder model. 


\subsection{Uniaxial Creep Curve Fitting}

\section{Selection of Modelling Technique}

As described in section 2.6, two possible modelling techniques were considered for the current work. The Theta projection method (equation 6.1) has proven reliability and accuracy for extrapolation, see for example [Evans'82], [Loghma.'96], [Hayhur.'85], [Taylor'90]. It also has the advantage of being a relatively straightforward strain vs. time relationship. The alternative method is the Continuum Damage Mechanics Model (CDM) which has also shown some success in predicting the behaviour of low alloy steels, and has been much used at the JRC Petten e.g. [Cane'82], [Braam'94], [Dunne'90], [Hayhur.'84], [Church'92]. The attractiveness of the CDM approach is the potential to relate the arbitrary damage parameter to some form of physical damage actually occurring in the material. Several similar forms of the equations exist, with equation 6.2 showing those proposed by Dunne [Dunne'90].

$$
\begin{aligned}
& \varepsilon=\theta_{1}\left(1-\exp \left(-\theta_{2} \mathrm{t}\right)\right)+\theta_{3}\left(\exp \left(\theta_{4} \mathrm{t}\right)-1\right) \\
& \frac{\mathrm{d} \varepsilon}{\mathrm{dt}}=\mathrm{Kt}^{-\mathrm{m}}\left(\frac{\sigma}{1-\omega}\right)^{n}, \quad \frac{\mathrm{d} \omega}{\mathrm{dt}}=\mathrm{At}^{-\mathrm{m}} \frac{\sigma^{\nu}}{(1-\omega)^{\phi}}
\end{aligned}
$$

\section{Constant Load / Constant Stress Considerations}

Both of the above mentioned models are normally used to fit creep curves determined under constant stress, with a creep curve prediction being made for any given stress. However, the creep curves in the current work were all performed under the more common constant load conditions. Under constant load conditions, the stress is continuously rising with increasing strain. Fitting and extrapolating uniaxial constant load creep curves with the constant stress model does not cause too many problems. After all, both models are essentially just curve fitting routines, and the increase in stress with strain is the same for all the uniaxial specimens. The problem occurs when trying to predict the creep behaviour of pressurised tubular components from uniaxial data. The increase in stress with strain in tubes occurs faster than in uniaxial specimens, meaning that the uniaxial constant load curve fits cannot be simply mapped onto the stress-state in the tubular components. When constant stress uniaxial curves are available the problem disappears. Components whose stress state varies continuously with strain (ie pressurised tubes, constant load uniaxial specimens) can be modelled by splitting the life of the component into small increments of time during which constant stress conditions are 
applicable, and iterating over the lifetime. The smaller the increments, the more accurate the prediction. However, this option was not available for the current work due to the lack of constant stress data.

Instead, it is necessary to use a technique suggested by Hurst and Rantala [Hurst'98] which is essentially the reverse of the procedure described above. The uniaxial creep data is initially fitted by assuming that it is constant stress data. The model is then used to predict what the constant load creep curve would look like for the same initial stress by using the iterative approach mentioned above. This new curve will have a shorter lifetime. The parameters of the model are then adjusted until the constant load (iterative) prediction matches the original experimental creep data. Unfortunately, this method induces rather non-linear behaviour in the models, where changing one parameter tends to affect the behaviour of another. This makes the method difficult to optimise computationally. Nevertheless, simple trial and error can produce very good curve fits given some experience and patience.

\section{Theta Projection vs. CDM for the Current Work}

Another problem now arises: because of the non-linearity and difficulty to find the parameters under constant load, the number of parameters in the model becomes a major factor in its' selection. The theta method is normally considered to have only four 'theta' parameters. However, in practice, each theta is in fact a function of stress (for a given temperature), requiring two parameters to define it (equation 6.3).

$$
\theta_{i}=g_{i}(\sigma)=\exp \left(a_{i}+b_{i} \sigma\right)
$$

where $a_{i}$ and $b_{i}$ are parameters to be determined. There are therefore eight parameters $\left(a_{1-4}\right.$ and $\left.b_{1-4}\right)$ to be fitted when using the Rantala technique in conjunction with the theta projection method [Evans'82]:

$$
\frac{\mathrm{d} \varepsilon}{\mathrm{dt}}=\mathrm{g}_{1}\left(\sigma_{\text {true }}\right) \mathrm{g}_{2}\left(\sigma_{\text {true }}\right) \exp \left\{-\mathrm{g}_{2}\left(\sigma_{\text {true }}\right) \mathrm{t}\right\}+\mathrm{g}_{3}\left(\sigma_{\text {true }}\right) \mathrm{g}_{4}\left(\sigma_{\text {true }}\right) \exp \left\{\mathrm{g}_{4}\left(\sigma_{\text {true }}\right) t\right\}
$$

where $g_{i}(\sigma)$ is defined in equation $6.3, \sigma_{\text {true }}=\sigma_{0} \cdot \exp (\varepsilon)$ with $\sigma_{0}$ equal to the initial stress and $\varepsilon$ as the true strain (for uniaxial creep).

The CDM model exists in several forms. At the JRC, the most commonly used version is shown in equation 6.2. Replacing $\sigma$ with $\sigma_{0}(1+\varepsilon)$ for uniaxial creep, where $\varepsilon$ is absolute strain converts the model to the constant load version. There are now only six parameters to find. 
Six parameters are still too numerous to fit by trial and error in such a non-linear system. However, it has been found that the value of ' $\mathrm{m}$ ' does not change significantly between constant load and constant stress predictions. In addition, $\mathrm{K}$ and $\mathrm{n}$ are closely related to the Norton law, and $\mathrm{n}$ should be greater than or equal to $v$, otherwise the failure strains will increase with decreasing stress, which is generally not the case.

Given these considerations, it was decided to opt for the CDM approach in the current work. Ideally, constant stress data would have been available, or a computerised solver could have been made to allow easier determination of the model parameters when using the Rantala method. This was beyond the scope of the current work. It was also hoped that the damage parameter could be related to some form of physical damage (i.e. hydrogen attack cavitation) occurring in the material.

\section{CDM Time Dependent Primary Creep Approach}

Equations 6.2 have been criticised for using the time dependent factor $t^{-\mathrm{m}}$ to account for primary creep. Primary creep is normally considered to be caused by strain hardening effects in the material, independent of time. However, the $t^{-\mathrm{m}}$ approach has been widely used because it allows the CDM coupled equations to be solved for constant stress conditions. In the current work, this makes little difference, since the equations will be solved numerically anyway to account for constant load. In addition, the lifetimes of various tests under different stresses will vary by large amounts. This means that the function $t^{-m}$ will have widely different values in different creep curves of different lifetimes. The result is that the model becomes over-sensitive to the ' $\mathrm{m}$ ' parameter, with small changes in $\mathrm{m}$ causing many of the other model parameters to be heavily re-adjusted to new values to maintain good curve fits.

\section{CDM Tertiary Only Approach}

One commonly used alternative is the tertiary only form, in which the $\mathrm{t}^{-\mathrm{m}}$ is absent. This is the original form of the equations and it assumes that there is no primary creep. Changing to this form has several advantages. For example, $\mathrm{K}$ and $\mathrm{n}$ now refer theoretically to the Norton law constants. This now leaves only three parameters to be found, which is relatively easy. However, in the current work it was found to be extremely difficult to fit the slope of the rupture curve unless either $v>n$ or $K$ and $n$ were changed from the Norton law constants. If $v>\mathrm{n}$ then the failure strains increase with decreasing stress which is generally not the case. The only alternative was changing $\mathrm{K}$ and $\mathrm{n}$ from the Norton constants, which eliminated one of the 
simplifying factors for using the tertiary only model. In addition, the tertiary only model does not truly capture the creep curve shape.

\section{CDM Strain Dependent Primary Creep Approach}

The third approach, suggested here, is to replace the $\mathrm{t}^{-\mathrm{m}}$ for primary creep with $\varepsilon^{-\mathrm{m}}$ as shown in equations $6.5 \mathrm{a}$ and $\mathrm{b}$. This is more realistic, as it now implies that primary creep is controlled by strain hardening events. In addition, the strain range in each creep curve is similar, meaning that each creep curve is affected in a similar way by the $\varepsilon^{-m}$ function. This makes the model more stable with respect to the $\mathrm{m}$ parameter, making it easier to fit all the other parameters too. The main problem with this method is that the equations cannot be solved for constant stress conditions. However, in the current work, numerical iterative solutions are being used anyway, so this does not hinder at all the use of the model.

$$
\begin{aligned}
& \frac{\mathrm{d} \varepsilon}{\mathrm{dt}}=\frac{K \sigma^{\mathrm{n}}}{(1-\omega)^{\mathrm{n}}} \varepsilon^{-\mathrm{m}} \\
& \frac{\mathrm{d} \omega}{\mathrm{dt}}=\frac{A \sigma^{\nu}}{(1-\omega)^{\phi}} \varepsilon^{-\mathrm{m}}
\end{aligned}
$$

$\mathrm{K}, \mathrm{n}, \mathrm{m}, \mathrm{A}, \mathrm{v}, \phi$ are material parameters to be found, $\omega$ is the arbitrary damage parameter, $\varepsilon$ is strain, $t$ is time and $\sigma$ is stress. One of the best features of this model is that primary creep is accounted for by the $\varepsilon^{-m}$ function which implies a strain controlled hardening of the material during primary creep. This is more realistic than the $\mathrm{t}^{-\mathrm{m}}$ which has been used in the past [Dunne'90].

\section{Parameter Fitting}

Equations $6.5 \mathrm{a}$ and $6.5 \mathrm{~b}$ need to be solved together to find a relation between strain and time. In addition, the true stress under constant load conditions for uniaxial creep is approximated by the expression:

$$
\sigma=\sigma_{0}(1+\varepsilon)
$$

where $\sigma_{0}$ is the initial stress. This needs to be substituted for the stress term in equations $6.5 \mathrm{a}$ and $b$. Analytical solution of the equations is not possible, so an iterative numerical solution is used. Ideally, a computerised solver would be able to find the six material parameters such that the error between the predicted creep curves and the experimental data is minimised. However, 
due to the non solvability of the equations, and the high amounts of non linearity when using constant load conditions, this was not considered feasible within the scope of the current work. Instead, a "manual" or trial and error fitting procedure was developed, based on a similar technique suggested by Rantala [Hurst'98], and is shown in Appendix C.

\section{Curve Fits}

Using the techniques in Appendix $\mathrm{C}$, the following parameter sets were found to give good fits of the uniaxial creep curves for bott air and hydrogen environments (table 6.1). Also included in the table is the damage level at failure $\omega_{\mathfrak{f}}$, which is not necessarily taken as 1 (see Appendix C).

\begin{tabular}{|c|c|c|}
\hline Parameters & Creep in Air & Creep in Hydrogen \\
\hline $\mathrm{K}$ & $4.9 \times 10^{-15}$ & $2.3 \times 10^{-15}$ \\
\hline $\mathrm{A}$ & $4.9 \times 10^{-16}$ & $5.5 \times 10^{-15}$ \\
\hline $\mathrm{n}$ & 6.2 & 6.2 \\
\hline$V$ & 6.188 & 5.75 \\
\hline$\phi$ & 2.2 & 3.7 \\
\hline $\mathrm{m}$ & 0.6 & 2 \\
\hline$\omega_{\mathrm{f}}$ & 0.62 & 0.853 \\
\hline
\end{tabular}

Table 6.1: CDM parameters for uniaxial creep curve fits in air and in hydrogen

The fitted creep curves and the rupture curves are compared with the experimental data in figures 6.1 - 6.4. Curve fits are generally good, with excellent fitting of the rupture plot in air being achieved. The hydrogen curve fits suffer mainly from the fact that the strain to failure does not decrease with increasing test time to the same extent as the experimental data. It was extremely difficult to fit both the strain to failure and the time to failure in this case. Similar problems were encountered when using the tertiary-only form of the CDM model, and it is thought that the simplistic damage evolution equation may be responsible for this inaccuracy. Despite this, the curve fits are still reasonable, and accurate prediction of the strain above a few percent is not one of the most pressing issues in assessing the lifetime of industrial components.

Initially, the hydrogen curves were fitted by assuming that the basic material properties remained unchanged from creep in air, and that only the damage evolution was different. This meant that $K, n$ and $m$ were kept constant and only $A, v$ and $\phi$ were changed. However, using this method it was not possible to fit well the hydrogen curve shapes. One of the main changes 


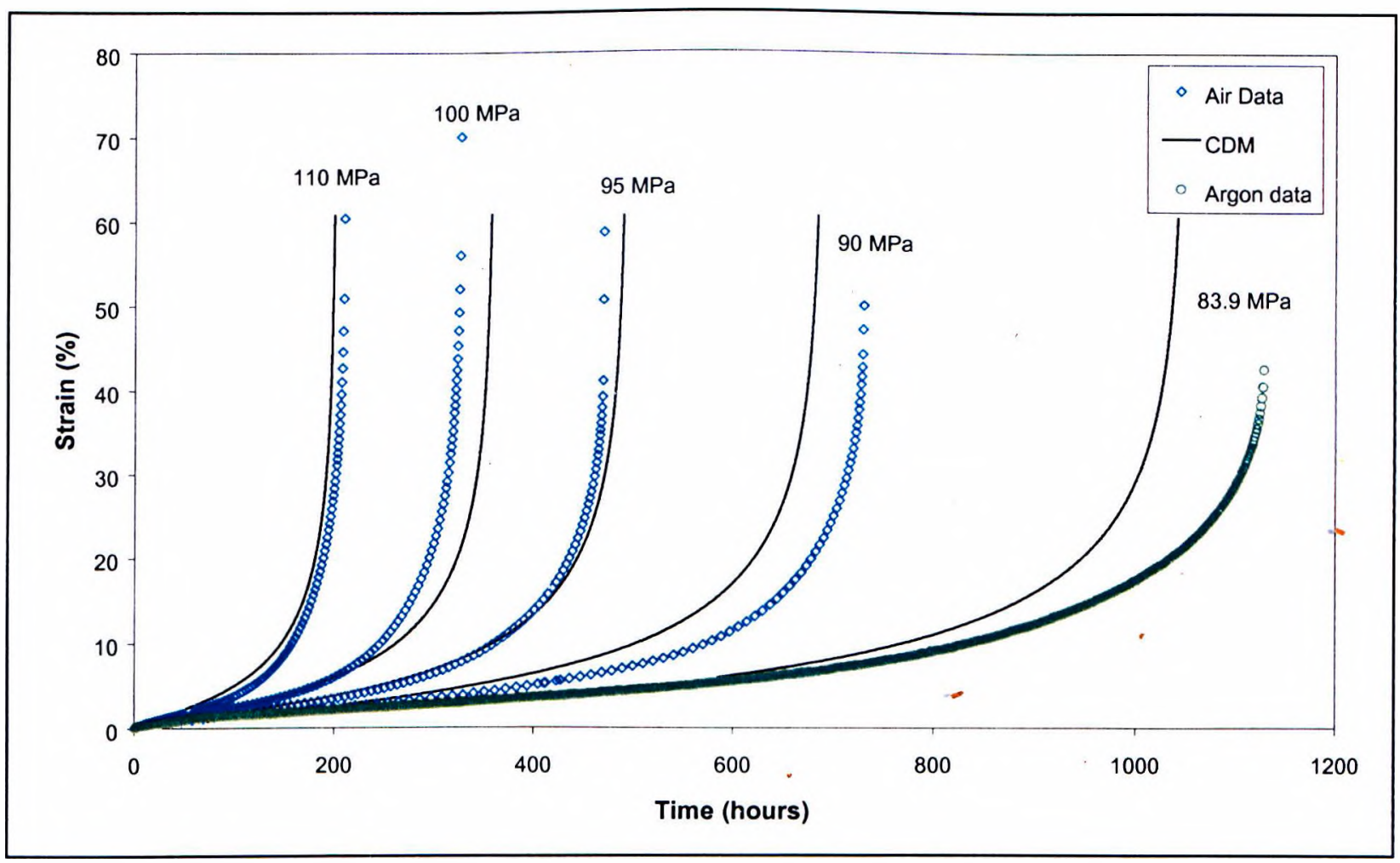

Figure 6.1: CDM fits of uniaxial creep data in an air environment. Also included is a creep test performed under in argon

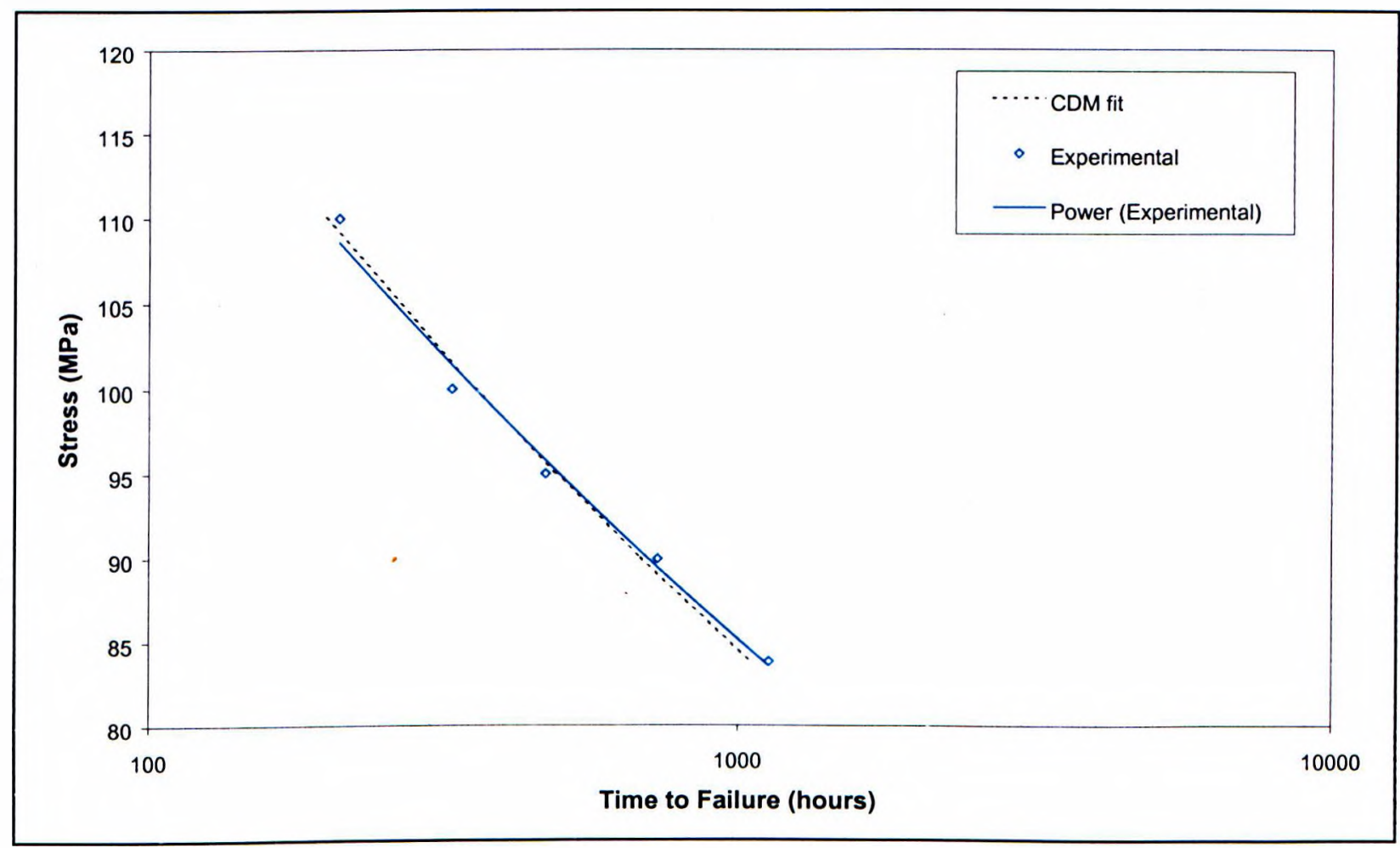

Figure 6.2: CDM fit of stress rupture plot for uniaxial creep in an inert environment.

appears to be in the amount of primary creep, with hydrogen tests showing greater amounts than tests in air. This meant that ' $\mathrm{m}$ ' had to be changed which in turn meant that some of the other parameters i.e. ' $\mathrm{K}$ ' also had to be changed. This also had the effect of altering the damage 
at failure, and explains why the parameter sets are so different between air and hydrogen. In addition, the damage evolution equation (6.5b) is the same for both air and hydrogen, whereas it is likely that the way in which the damage progresses under hydrogen attack is somewhat different to the situation in air.

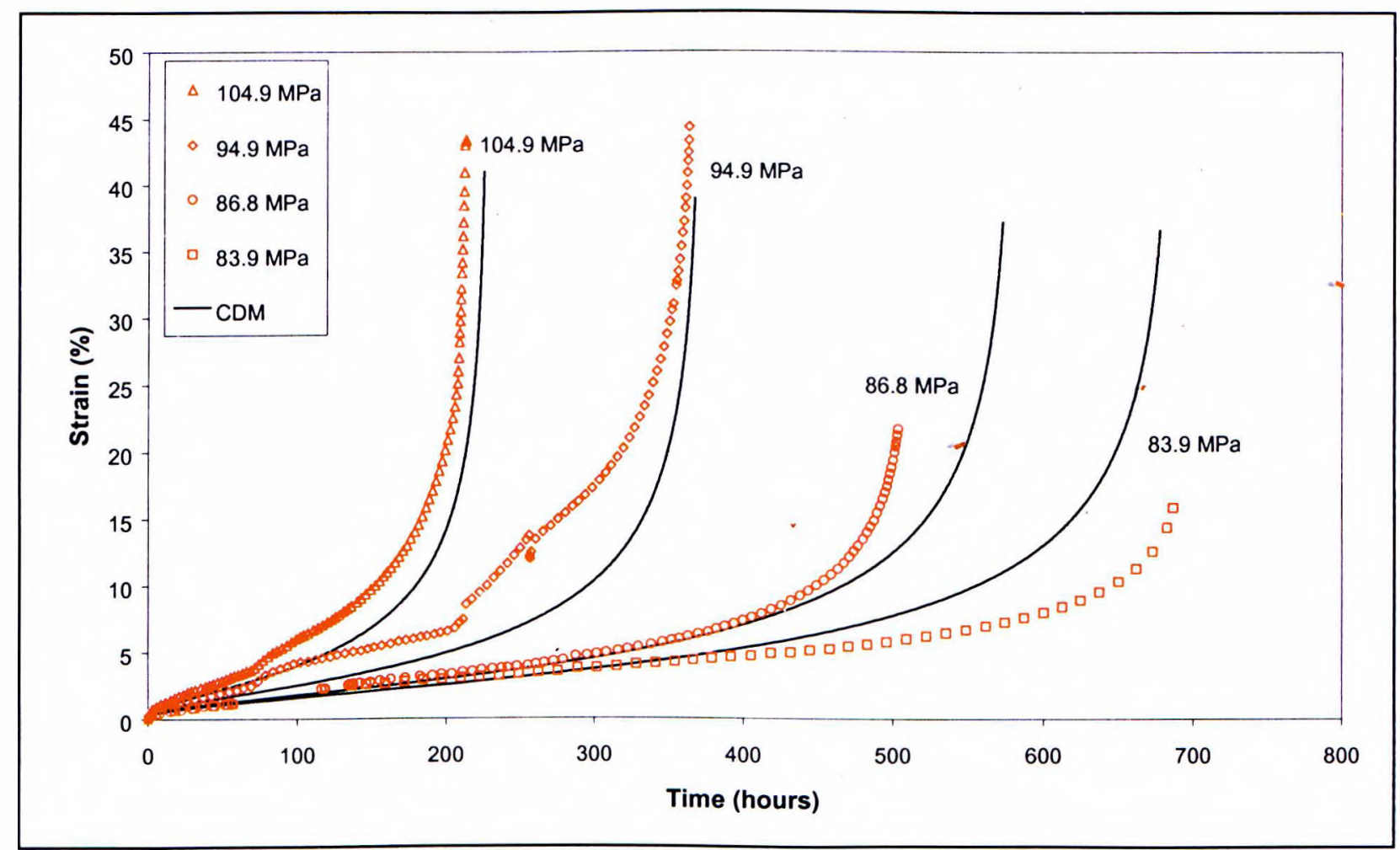

Figure 6.3: CDM curve fits for uniaxial creep tests in a 220bar hydrogen environment

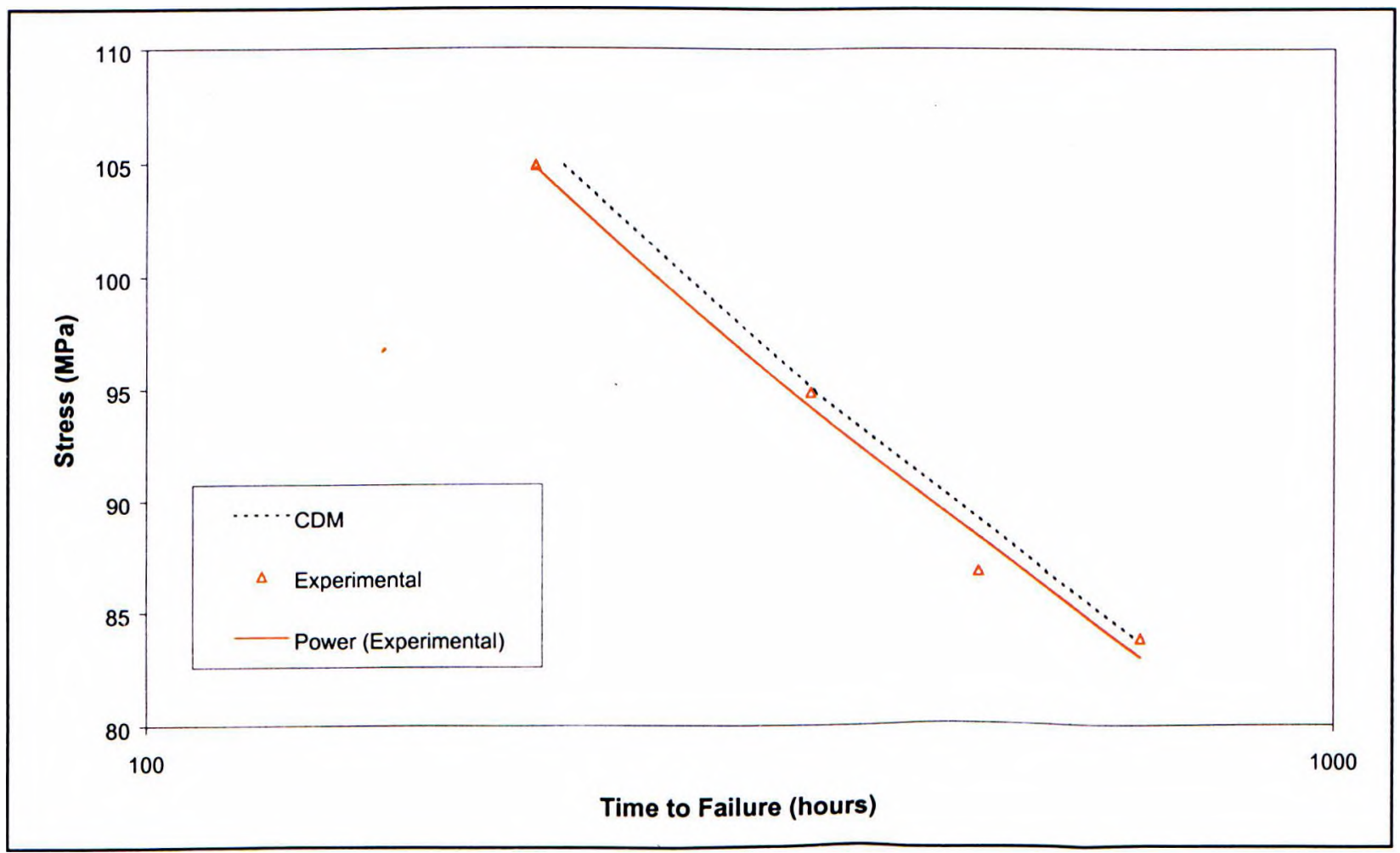

Figure 6.4: CDM fit of stress rupture plot for uniaxial creep in 220bar hydrogen 


\section{Alternative Method For Hydrogen Curve Fits}

The damage caused by hydrogen attack can perhaps be considered as additional to the damage which occurs in uniaxial creep specimens tested in air. The damage parameter for air tests represents a variety of damage mechanisms which could include, for example, microstructural changes and non uniform necking. It is assumed that these damage processes will still operate under a hydrogen environment.

In hydrogen attack, the main form of damage is grain boundary cavitation. CDM models specifically for diffusional and creep growth of cavities exist in the literature [Cocks'82],[Ashby'84]. The damage evolution and strain rates predicted by these models could theoretically be added to the CDM model for creep in air. In this way, the damage associated with hydrogen attack is kept separate from the normal ductile damage, facilitating the relationship between metallographic cavity observations and the hydrogen attack damage parameter.

Initial trials of this method proved difficult to implement successfully, and have not been pursued further in this thesis. 


\subsection{Predictions of Tubular Hoop Strain Creep Behaviour}

The CDM model is readily adapted to the multiaxial stress case, its form changing slightly as shown below:

$$
\begin{aligned}
& \frac{\mathrm{d} \varepsilon_{1}}{\mathrm{dt}}=\frac{3}{2} \frac{\mathrm{K} \sigma_{\mathrm{MSDC}}^{\mathrm{n}-1}}{(1-\omega)^{\mathrm{n}}}\left[\sigma_{1}-0.5\left(\sigma_{2}+\sigma_{3}\right)\right] \varepsilon_{1}^{-\mathrm{m}} \\
& \frac{\mathrm{d} \omega}{\mathrm{dt}}=\frac{\mathrm{A} \sigma_{\mathrm{MSRC}}^{\mathrm{v}}}{(1-\omega)^{\phi}} \varepsilon_{1}^{-\mathrm{m}}
\end{aligned}
$$

where $\sigma_{\mathrm{MSDC}}$ is the multiaxial stress deformation criteria and $\sigma_{\mathrm{MSRC}}$ is the multiaxial stress rupture criteria. $\sigma_{1}, \sigma_{2}$, and $\sigma_{3}$ are the principal stresses, namely the hoop, axial and radial stress in the tube, and $\varepsilon_{1}$ is the hoop strain. For most cases the Von Mises stress is considered most meaningful in controlling deformation [Johnso.'51], and is therefore used as the MSDC. The Maximum Principal Stress (MPS), or in this case the hoop stress, is often used for the MSRC, although many forms of the MSRC exist, including those which include a contribution from both the Von Mises stress and the MPS [Hayhur.'84], [Cane'81b]. By keeping the parameters $\mathrm{K}, \mathrm{A}, \mathrm{n}, \mathrm{v}, \phi$, and $\mathrm{m}$ the same as the uniaxial case, it should be possible to predict the creep behaviour of the tubes by careful selection of the MSDC and MSRC alone.

\section{Thick Wall and Thin Wall Stress Distributions}

\section{Thin Walled Tubes}

To find the MSDC and MSRC, the hoop and Von Mises stresses need to be found for the tubular geometry in question. For thin walled tubes, the solutions are simple, namely:

$$
\sigma_{\mathrm{h}}=\frac{\mathrm{Pd}_{\mathrm{m}}}{2 \mathrm{t}}, \quad \sigma_{\mathrm{i}}=\frac{\sigma_{\mathrm{h}}}{2}, \quad \sigma_{\mathrm{r}}=0, \quad \sigma_{\mathrm{VM}}=\frac{\sqrt{3}}{2} \sigma_{\mathrm{h}}
$$

where $\sigma_{\mathrm{h}}, \sigma_{\mathrm{a}}, \sigma_{\mathrm{r}}, \sigma_{\mathrm{VM}}$ are the hoop, axial, radial and Von Mises stresses in the tube, $\mathrm{P}$ is the internal pressure, $d_{m}$ is the mean diameter, and $t$ is the wall thickness. It is assumed that there is no stress distribution across the wall of the tube. This makes the choice of the MSDC and MSRC rather simple. These equations have been quoted as being valid only when the mean diameter to thickness ratio is greater than at least 10:1 [Hender.'82]. In the current work, the ratio is only 8.72 . However, industrial hydrocracking vessels may have dimensions of about $280 \mathrm{~mm}$ thickness by $3600 \mathrm{~mm}$ diameter which would class them as thin walled e.g.[Nuovo]. 


\section{Thick Walled Tubes}

Equations 2.30 in the literature review give the stress distributions in thick walled tubes during steady state creep (assuming the Norton law is representative of creep). In this case, there is a distribution of stresses across the tube wall. A position must then be chosen from where the MSDC and the MSRC can be taken. The skeletal point is often used as it is independent of the creep exponent ' $n$ ' for the given material (where $n$ is derived from the Norton law and is not necessarily equal to $\mathrm{n}$ in the CDM equations), and does not change during stress redistribution from the elastic to creep stress states. Equations 2.30 only give the stress distributions during steady state creep, whereas in reality the stress distribution may change during primary "and tertiary creep [Johnso.'61], and due to variable damage in the tube wall. Thus, when calculating the damage parameter $\omega$, it is necessary to assume that damage is homogenous across the tube wall. When using finite element methods, the strain rate and damage rate can be calculated at individual points within the tube wall for the local stress conditions. The work in this thesis is however based upon simpler predictive methods, using a single representative stress for deformation and one for rupture.

\section{Argon Pressurised Tubes}

\section{Application of the Model}

The six material parameters are already known for uniaxial creep in air. The philosophy of the modelling approach states that the parameters are the same in the multiaxial case, with only the stress terms changing. Thus, the first step in predicting the tube behaviour is to choose the MSDC and MSRC. These are entered into equations 6.7 and 6.8, and an iterative procedure is used to predict the creep curve shape for the hoop strain. The procedure is very similar to that of the uniaxial case (Appendix $C$, step 2) except that as the tube strains the true stress must be recalculated from the new tube dimensions, and cannot follow the simple $\sigma=\sigma_{0}(1+\varepsilon)$ equation which is used for uniaxial creep. For the thick walled case, the steady state stress distribution is assumed to be present from the beginning of the test. This assumption can be borne out with finite element simulations which show that the redistribution time from elastic to creep stress distributions is relatively rapid (figure 6.5) (see also [Taira'68], [Johnso.'61]).

\section{Thin Walled Predictions}

Assuming the stresses in the tube could be accurately described by the thin wall equations, predictions were made by assuming that the MSDC was the Von Mises stress, and the MSRC 


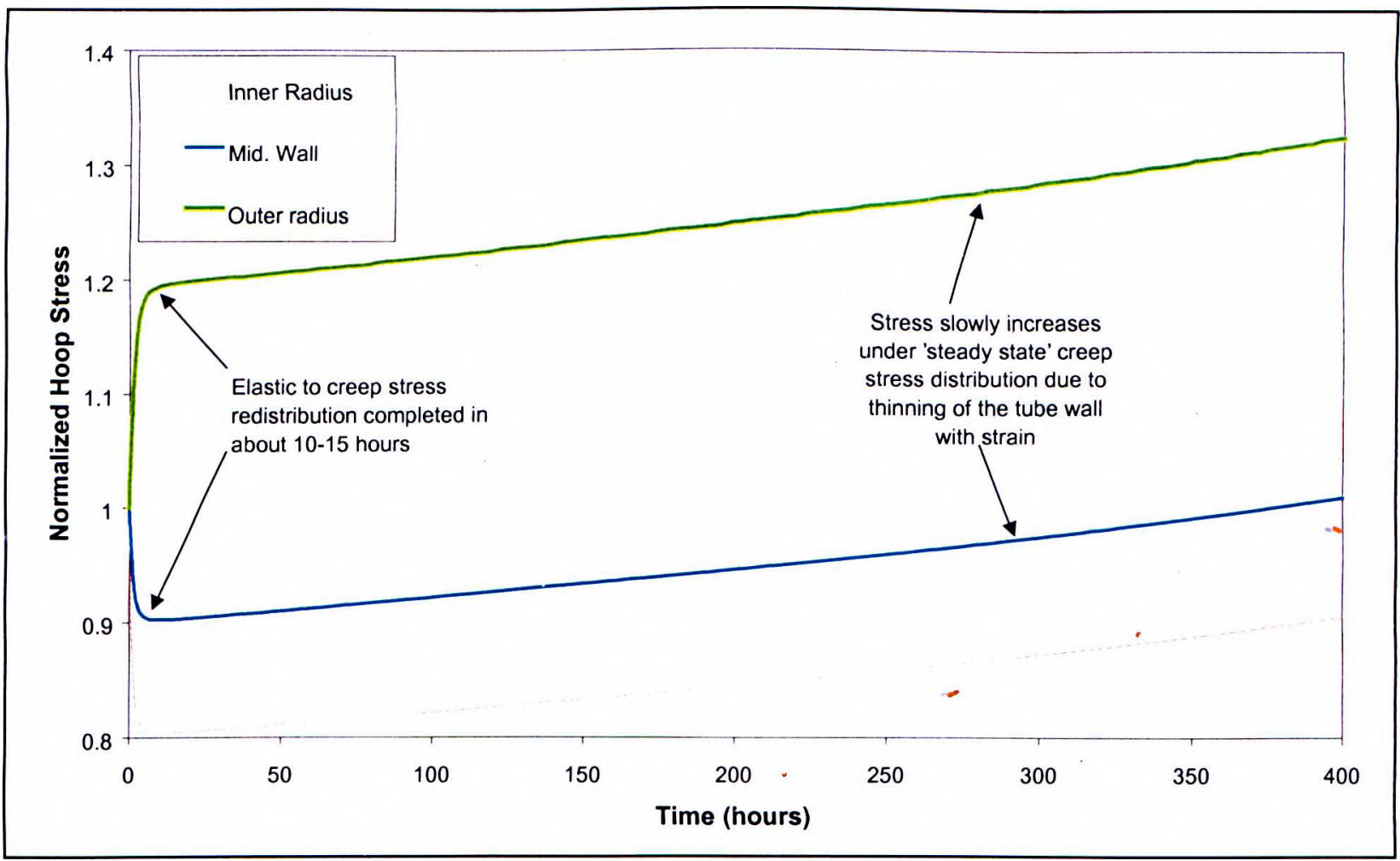

Figure 6.5: Finite element simulation showing hoop stress redistribution from the initial elastic to creep distributions for the current material and specimens. The above curves assume the Norton law and an internal pressure of $24 \mathrm{MPa}$

was the MPS (similar to the simple reference stress derived in section 5.3). Some adjustment can be made to the MSRC, which is often taken as a combination of MPS and Von Mises stresses. The simplest form of combination is shown below [Hayhur.' 84 ]:

$$
\sigma_{\mathrm{MSRC}}=\alpha \cdot \sigma_{\mathrm{MPS}}+(1-\alpha) \cdot \sigma_{\mathrm{VM}}
$$

where $\alpha$ is a constant between 0 and 1 . To avoid using the extra parameter $\alpha$, it was decided to leave the MPS as the controlling MSRC (ie. $\alpha=1$ ). It was found that the mean diameter hoop stress equation produced creep rates which were too high, and failure times which were too low. As a result, the thin wall equations were modified so that the mean diameter hoop stress was replaced by the inner diameter hoop stress shown below:

$$
\sigma_{\text {id.hoop }}=\frac{P_{i}}{2 t}
$$

where $d_{i}$ is the inner tube diameter. This stress has also been used for predicting the behaviour of other 2.25Cr-1Mo steel tubes pressurised with argon [Church'92]. The reason for its success may be that it approximates quite closely to the thick wall Von Mises skeletal point stress, whereas the mean diameter hoop stress is much higher (for example, for a tube pressurised with 
220bar, the mean diameter hoop stress is $11 \mathrm{MPa}$ higher than the inner diameter hoop stress). The predicted hoop strain curves are plotted with the experimental data in figure 6.6. The corresponding predicted pressure-rupture curve is shown in figure 6.7. Good predictions are obtained, particularly for the early parts of the creep curves where strain is relatively low. Other predictions of $2.25 \mathrm{Cr}-1$ Mo pressurised tube creep behaviour found that the best fits were obtained when $\alpha$ (equation 6.10) was around 0.5 [Church'92], although in that case the material heat treatment was different, and a different form of the CDM model was used.

\section{Thick Walled Predictions}

The thick walled predictions are somewhat more complex, as a variety of choices exist for the MSDC and the MSRC. Common choices of stresses include the skeletal point.stresses, the inner diameter Von Mises stress (position of maximum Von Mises stress), and the outer diameter hoop stress (position of maximum hoop stress). Given the ability to change the MSRC according to equation 6.10 means that there are a large variety of stress combinations which could be used to predict the tube behaviour. To minimise the amount of arbitrary choosing, the initial predictions were made using the skeletal point Von Mises stress for the MSDC (see section 5.3 for simple reference stress calculations) and the skeletal point MPS for the MSRC. The predicted curves are shown in figure 6.8. The predictions are quite good, particularly in the early part of the curves, although there is a trend to consistently overestimate the failure time which is demonstrated in the pressure-rupture plot (figure 6.9).

Figures 6.10 and 6.11 show that carefully choosing different MSDC and MSRC combinations can also result in very similar, good predictions. It suggests that the predictions are insensitive to the MSDC and MSRC. This is not the case however, since certain combinations do not work at all, for example, putting the MSDC as the outer radius Von Mises stress, and the MSRC as the inner radius MPS gives rupture lifetimes a factor of two too high. Thus there are a given number of MSDC-MSRC combinations which will work adequately well, which puts some doubt as to what are really the controlling multiaxial stresses. This is a major problems when using the thick wall stress distributions with simple models which do not take account of the local conditions in the tube in the same implicit way as finite elements. 


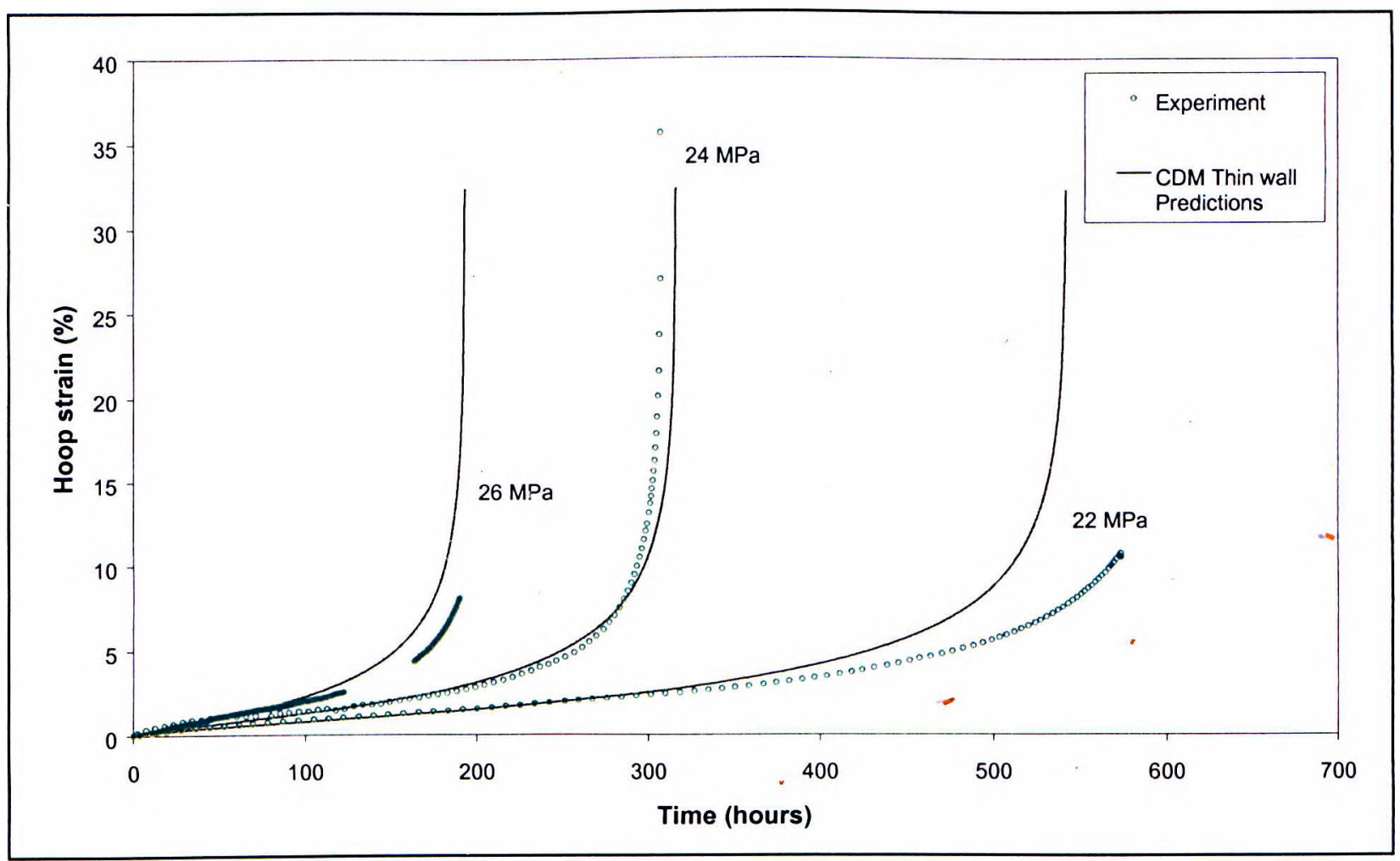

Figure 6.6: CDM predictions of argon pressurised tubes using thin wall assumption. The $M S D C$ is the Von Mises stress, and the MSRC is the MPS.

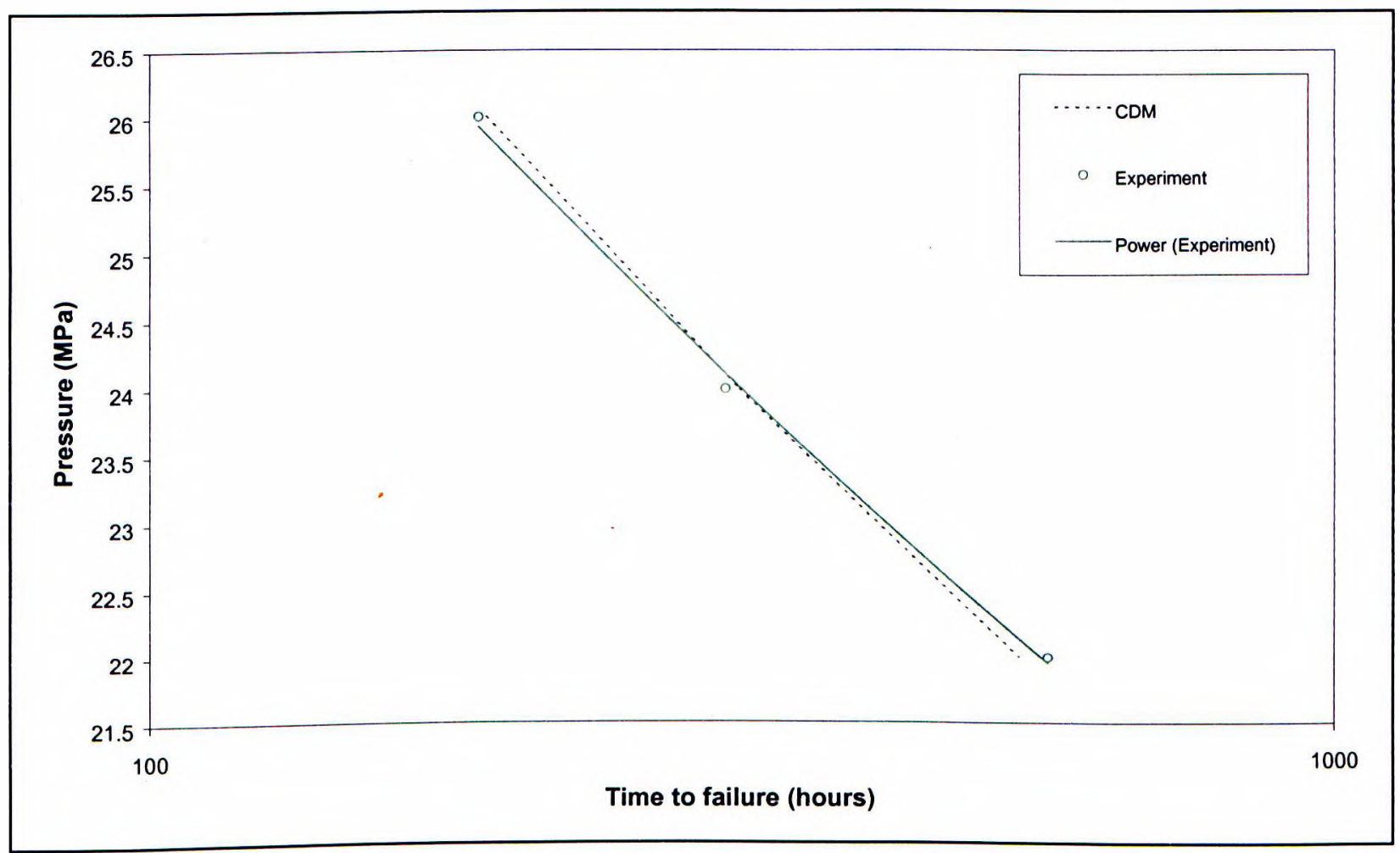

Figure 6.7: CDM thin wall prediction of pressure - rupture plot for tubes pressurised with argon 


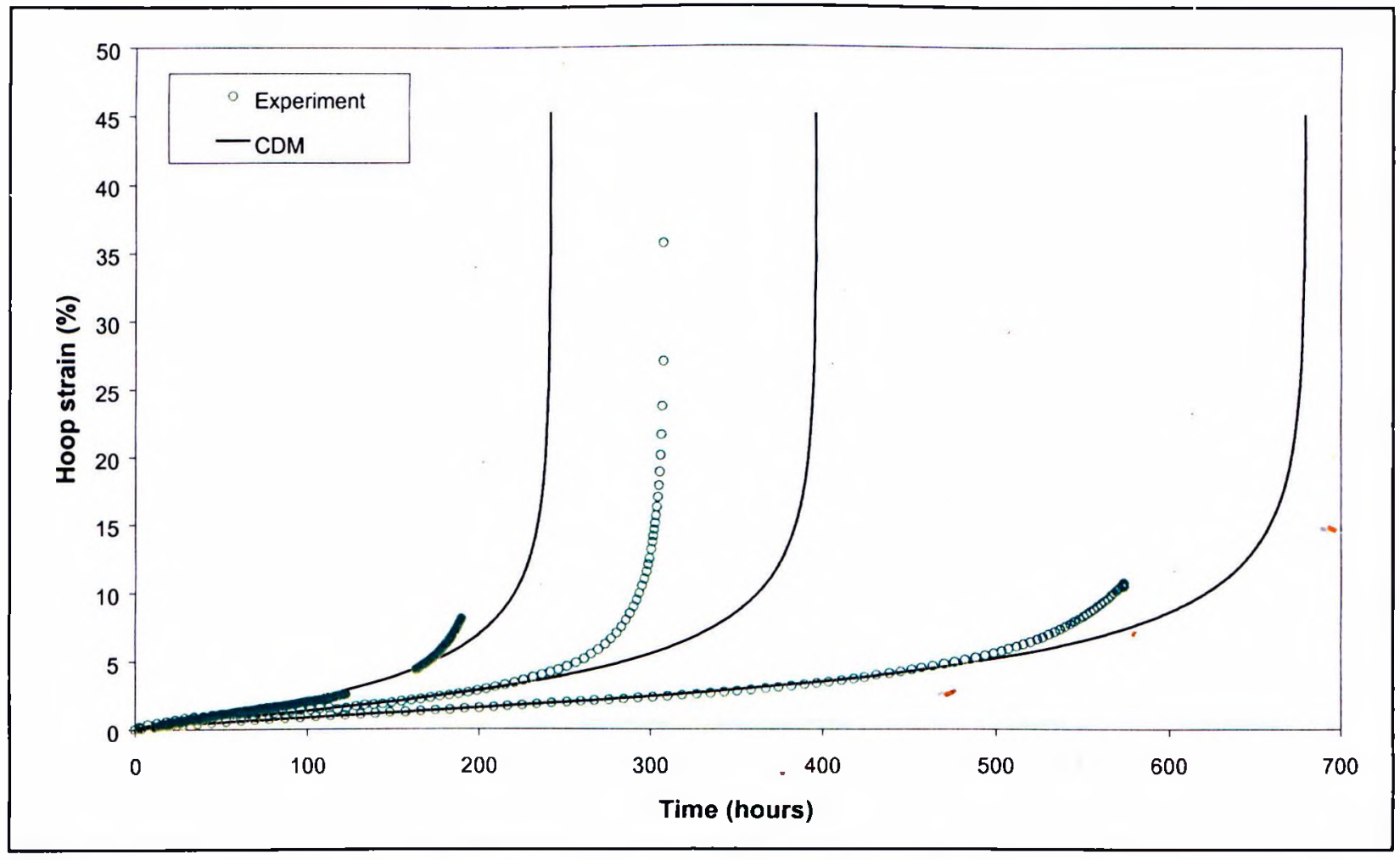

Figure 6.8: CDM predictions of argon pressurised tubes using thick wall assumptions. The MSDC is the skeletal point Von Mises stress, and the MSRC is the MPS at the skeletal point.

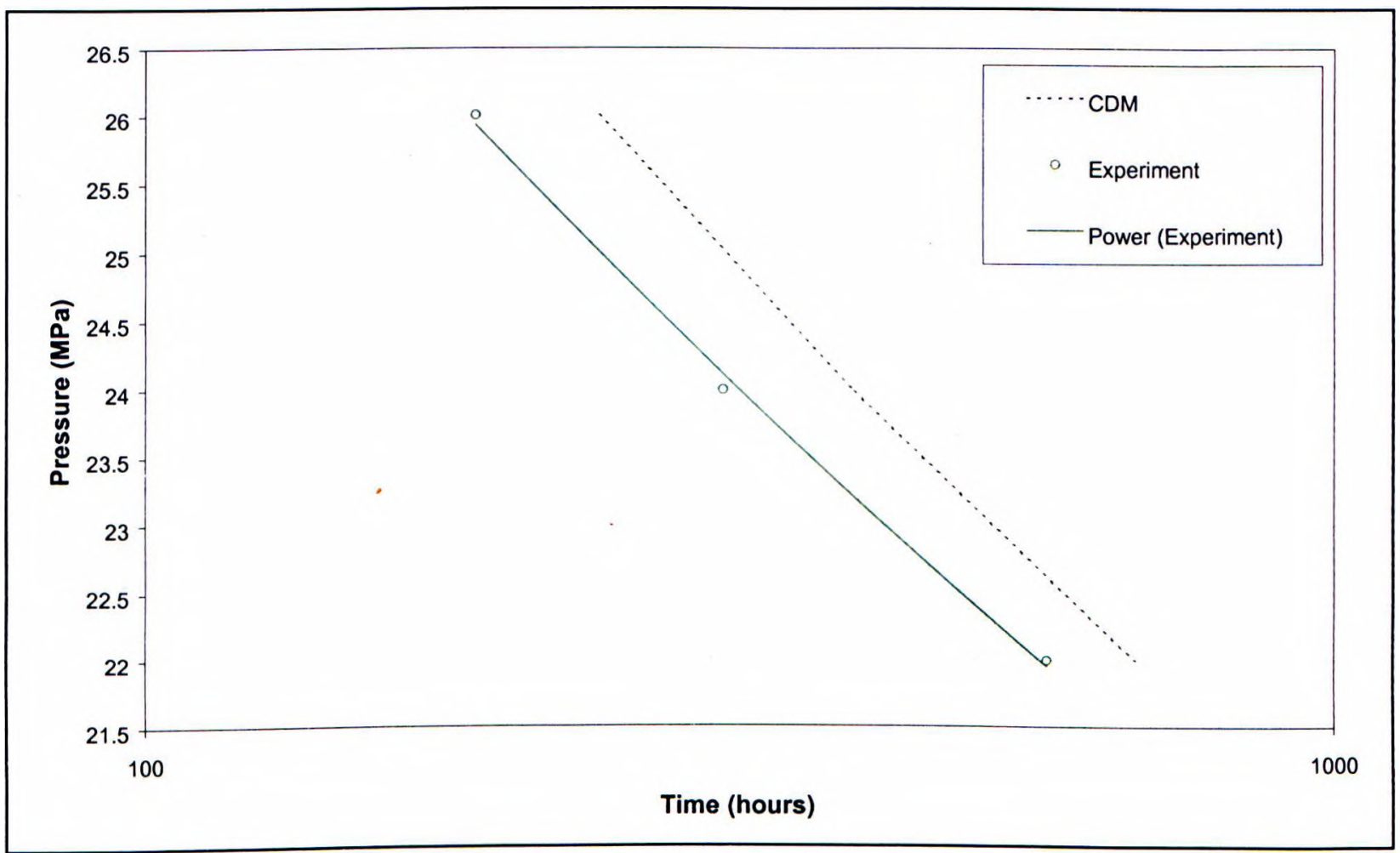

Figure 6.9: CDM thick wall prediction of pressure - rupture plot for tubes pressurised with argon 


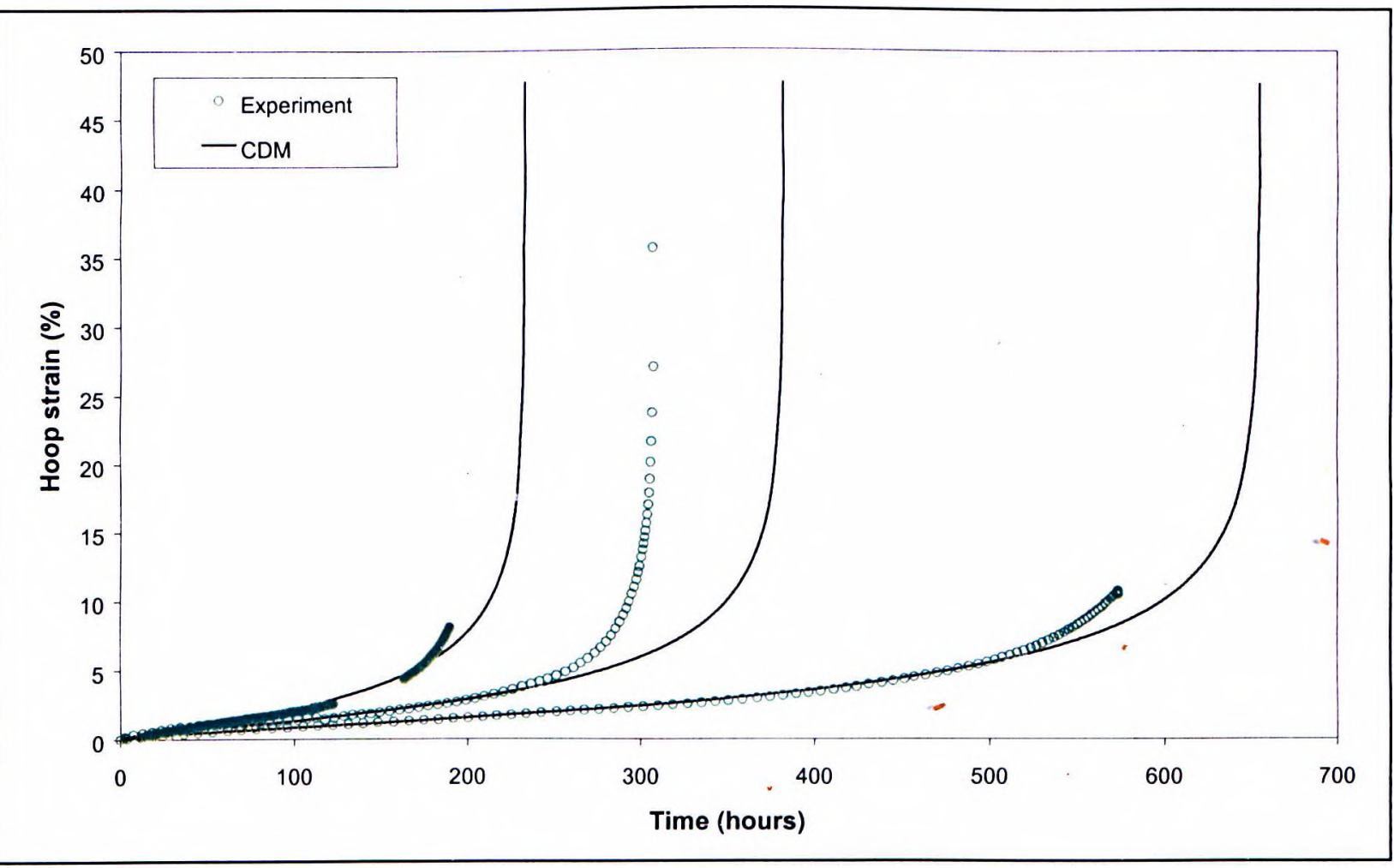

Figure 6.10: Altemative thick wall CDM prediction of Argon pressurised tubes. The MSDC is the inner radius Von Mises stress, and the MSRC is $0.32 x M P S+0.68 x$ Von Mises at the outer radius (i.e. $\alpha=0.32$ ).

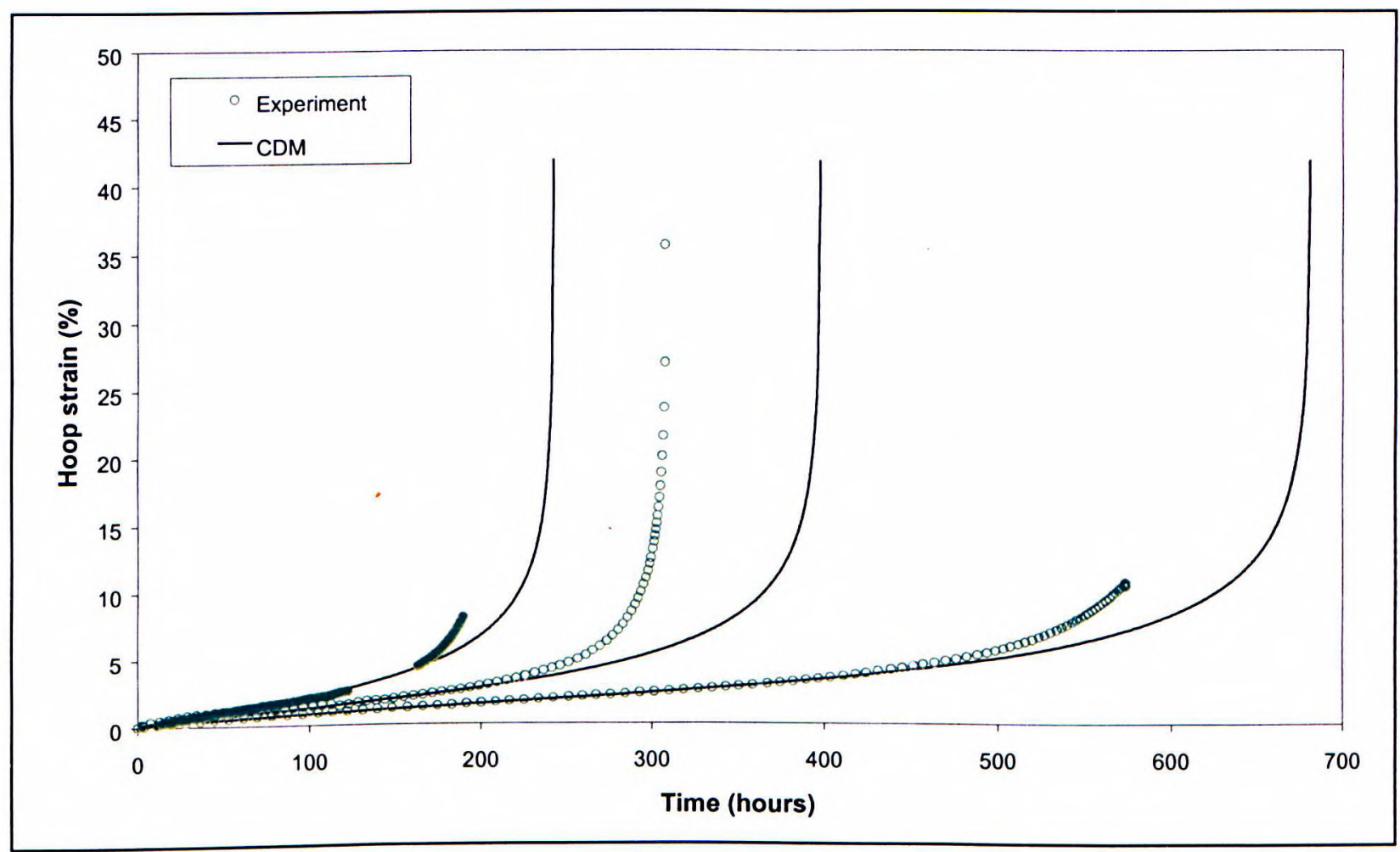

Figure 6.11: Alternative thick wall CDM prediction of Argon pressurised tubes. The MSDC is the inner radius Von Mises stress, and the MSRC the MPS at the skeletal point. 


\section{Hydrogen Pressurised Tubes}

\section{General Methodology}

The metallographic results in section 5.3 show that there is a gradient of hydrogen attack damage from the inner tube wall to the outer. During creep, this is expected to result in stress redistribution due to the different material properties across the tube wall, which must be considered in the model. There also exists a gradient of hydrogen concentration across the tube wall, being highest at the inside and low (zero to a first approximation) at the outer wall. Within the scope of the current work, it was only possible to determine material CDM parameters for uniaxial creep in two situations: without hydrogen, and under $22 \mathrm{MPa}$ hydrogen. These datà are used to approximate to the range of internal test pressures and hydrogen concentrations in the tubes by making the following assumptions:

1. Hydrogen attack kinetics at $22 \mathrm{MPa} \mathrm{H}_{2}$ (uniaxial çase) are similar enough to to the kinetics when using between 19.5 and $26 \mathrm{MPa} \mathrm{H}_{2}$ (tube test pressures) to avoid large errors in the modelling. According to the Nelson curves for $2.25 \mathrm{Cr}-1 \mathrm{Mo}$ steel, the difference in hydrogen attack with increasing pressure above a pressure of 14MPa is small [API'97]. In addition, methane pressure calculations suggest that the increase in methane pressure with hydrogen pressure becomes less as hydrogen pressure increases e.g. [VdBurg'96].

2. The damage gradient in the tubes can be simplified by assuming there is an inner region to a depth of $0.75 \mathrm{~mm}$ which can be considered attacked, and an outer region where the material properties are the same as those tested in air or argon. This depth corresponds approximately to the depth at which cavitation could be easily observed with the optical microscope in the tubes (section 5.3).

The resulting model consists of two concentric cylinders, the inner one which behaves according to the model parameters determined for uniaxial creep in hydrogen, and the outer behaving according to the parameters for creep in air. This is illustrated in figure 6.12. Solution of the model proceeds by calculating the expected strain rates in each cylinder. The stress in the outer and inner cylinders are then redistributed so that the strain rates of the two cylinders (or at least the strain rate at the interface between the two) is the same. Once the stress has been found, the damage evolution can be calculated and the next iteration step can follow. 


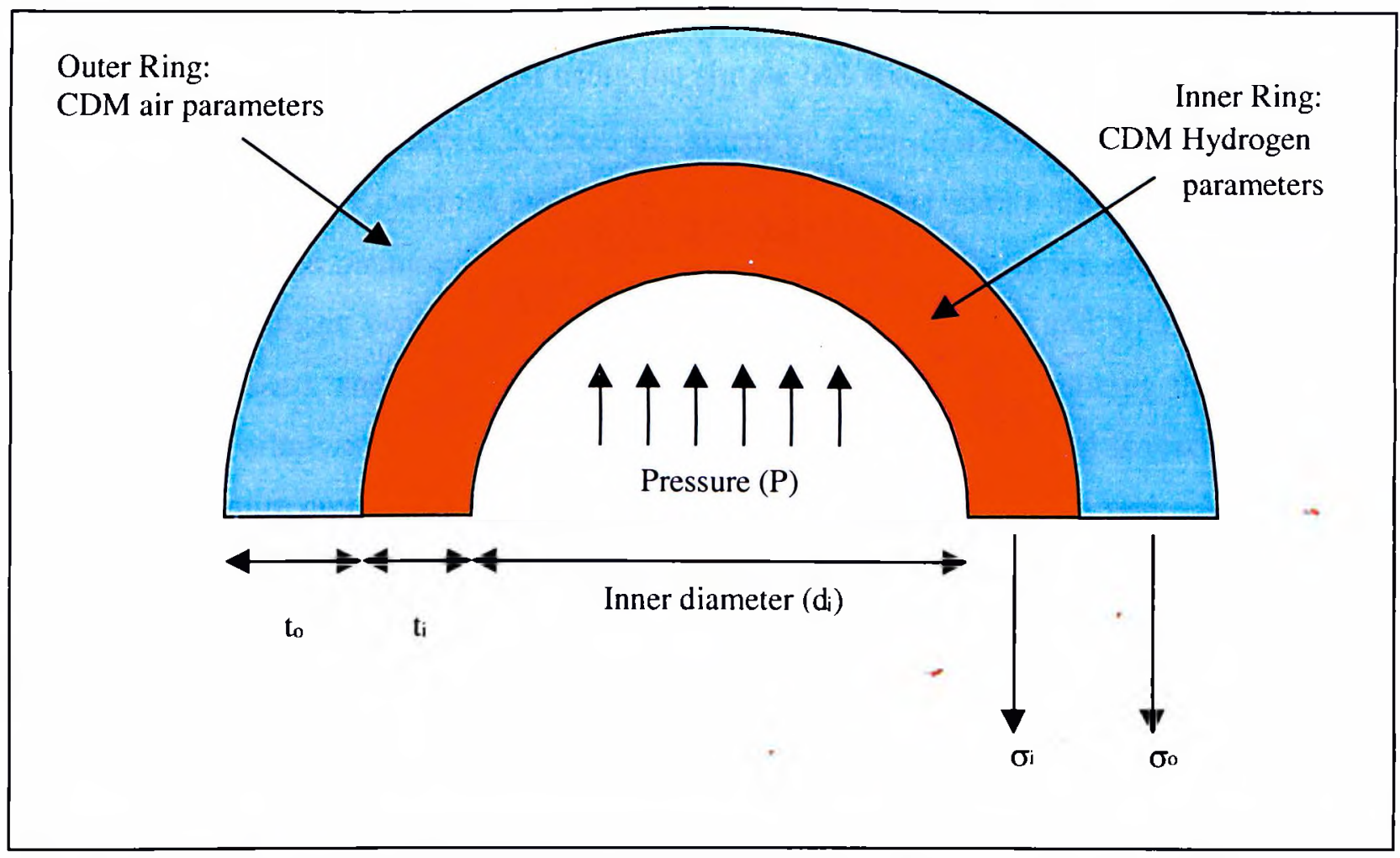

Figure 6.12: Simplification of the hydrogen pressurised tubes for modelling purpose. $\left(t_{0}\right.$ and $t_{i}$ refer to the thickness of the two concentric cylinders with $\sigma_{o}$ and $\sigma_{i}$ referring to the outer and inner cylinder hoop stresses respectively.)

\section{Thin Wall Concentric Cvlinders}

This method is essentially the same as that used by Taylor in the study of carburisation of alloy 800 tubes [Taylor'87]. The tube is split into two concentric rings as shown in figure 6.12. A balance of forces is approximated between the internal pressure (P) and the hoop stresses in the cylinder walls $\left(\sigma_{0}, \sigma_{\mathrm{i}}\right)$ :

$$
\text { P.d } d_{i}=2\left(\sigma_{i} t_{i}+\sigma_{o} t_{o}\right)
$$

Rearrangement gives:

$$
\sigma_{i}=\frac{\left(\frac{P d_{i}}{2}-\sigma_{0} t_{0}\right)}{t_{i}}
$$

The axial and Von Mises stresses are easily calculated from this using equations (6.9). Substituting equation 6.13 in the CDM expression for the strain rate of the inner cylinder (eqn.(6.7)) and using the hydrogen parameters gives the inner strain rate as a function of the outer cylinder stress, $\sigma_{0}$. Similarly, the strain rate for the outer cylinder (air parameters) can also 
be found a function of $\sigma_{\mathrm{o}}$. The strain rates in the inner and outer cylinders are then equalised by changing $\sigma_{0}$. This was accomplished using the simple MS Excel Goal Seek function. Thus the stress redistribution is calculated. A small increment of strain $(0.025 \%)$ is taken during which the stress distribution is assumed constant. Knowing the strain rate, a time increment can be calculated so that the increase in damage can be found for both the inner and outer cylinders from the CDM damage evolution equations. The time, strain, damage and tubular dimensions can then be updated, and the iterative procedure begins again by calculating the new stress redistribution. Failure is considered to occur when the critical damage level is reached in either cylinder. The entire procedure was automated using MS Excel spreadsheets with a Visual Basic Macro to trigger the Goal Seek function for each iteration.

The results from the thin wall concentric cylinder model are shown in figure 6.13. The thickness of the inner cylinder was taken as $0.75 \mathrm{~mm}$, the Von Mises stress was assumed to be the MSDC and the MPS was the MSRC for both cylinders. The resulting curve fits are reasonably good, although there is a tendency to overestimate the lifetime as shown in the predicted rupture plot. (figure 6.14).

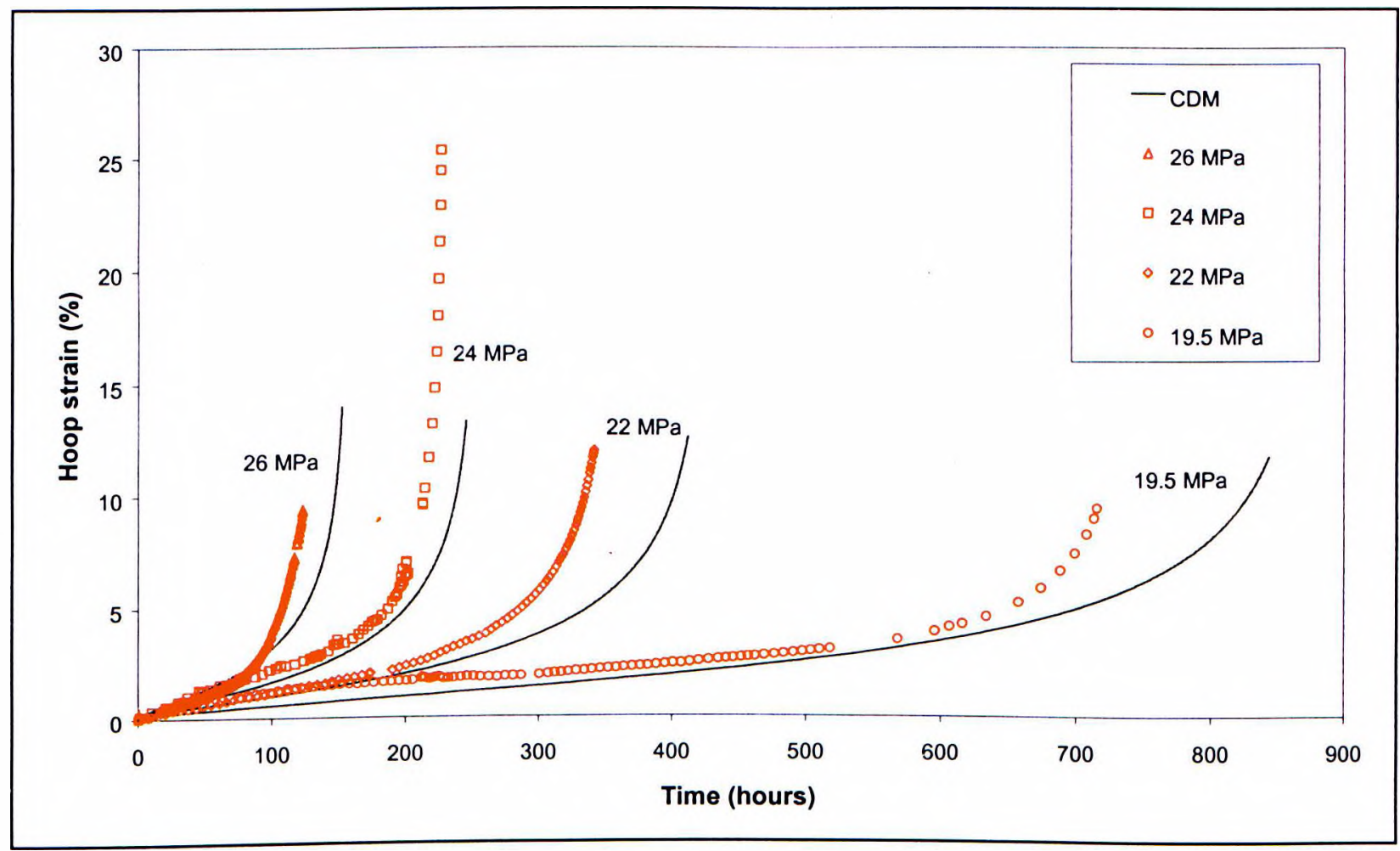

Figure 6.13: CDM predictions of hydrogen pressurised tubes using thin wall assumptions with the concentric cylinder model. The MSDC is the Von Mises stress, and the MPS is used for the MSRC. 


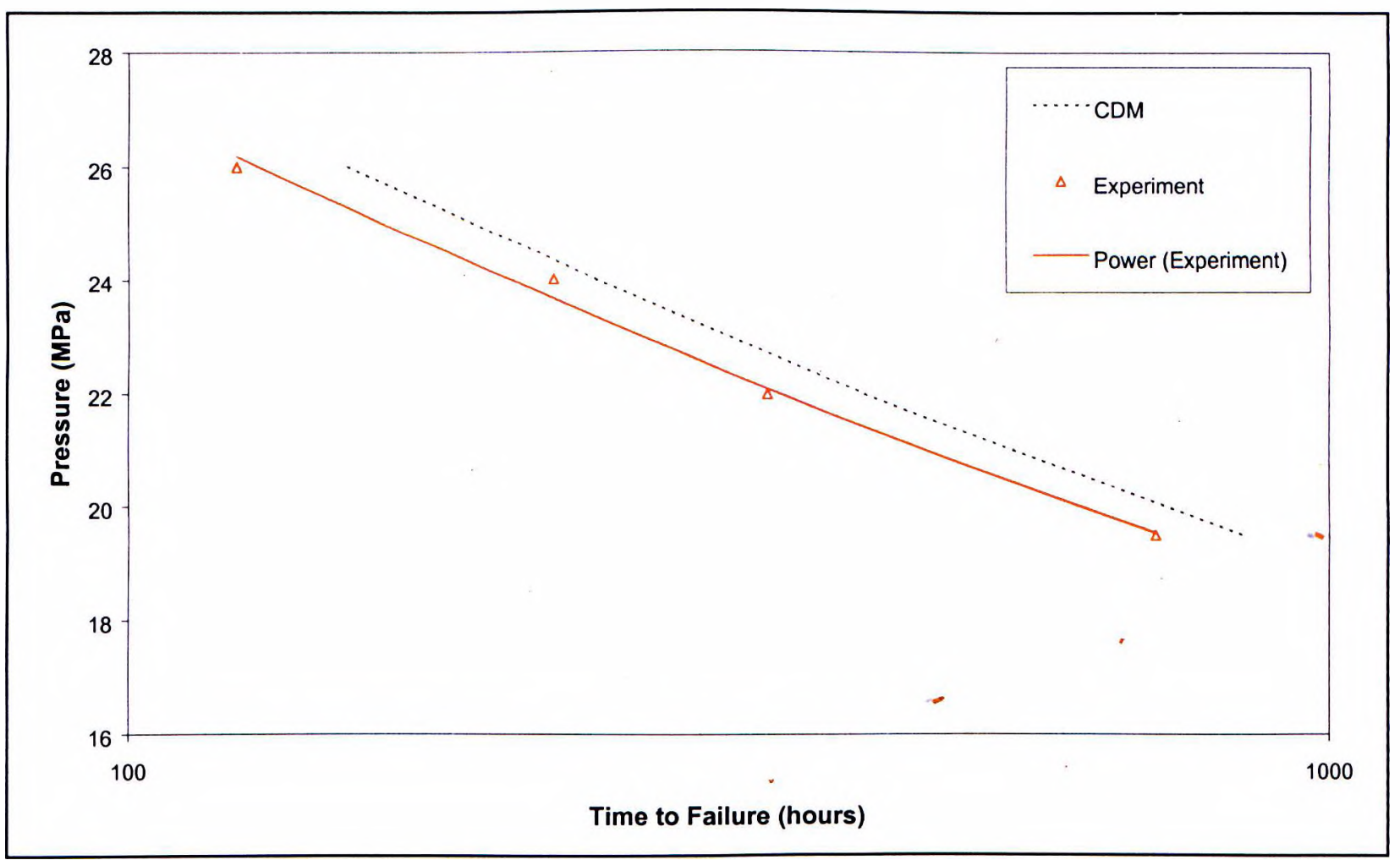

Figure 6.14: CDM predicted pressure-rupture plot for hydrogen pressurised tubes, using thin wall assumptions in the concentric cylinder model

The model has been validated by entering the CDM parameters for creep in air into both inner and outer cylinders. The resulting creep curve was the same as the prediction for argon pressurised tubes presented in figure 6.5.

\section{Thick Wall Concentric Cvlinders}

The thick wall method works in a somewhat more complex manner, although the principal of the concentric cylinders remains the same. The calculation procedure is given in Appendix D.

When using the thick wall model, there are many choices available for the input parameters. For example, the position of the MSDC and the MSRC in the inner and outer cylinder, and the type of MSRC in each cylinder (i.e. the $\alpha$ value from equation 6.10). There are a very wide range of possible combinations. To reduce the amount of arbitrary choice, initial predictions were based on the following assumptions:

- The MSDC is the Von Mises Stress at the skeletal point. This was used successfully for the argon pressurised tubes, and is a common MSDC. 


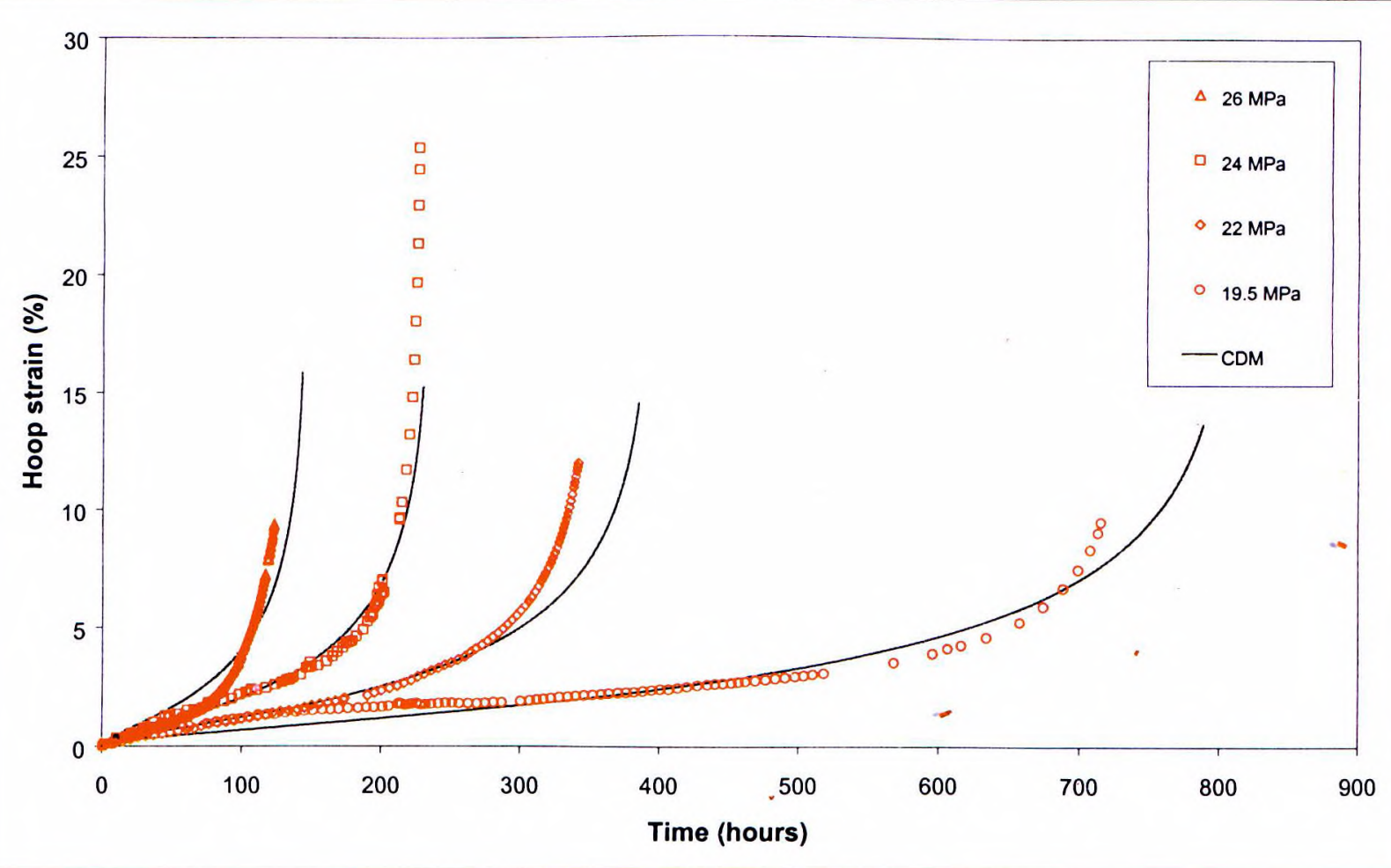

Figure 6.15: CDM predictions of hydrogen pressurised tubes using thick wall assumptions with the concentric cylinder model. The MSDC is the Von Mises stress at the skeletal point of each cylinder, the inner cylinder MSRC is the inner wall MPS, and the outer cylinder MSRC is the skeletal point MPS.

- The MSRC for the inner cylinder is taken at the inner wall to reflect the higher hydrogen concentration and damage there (surface decarburisation is neglected). The MPS is used because it was shown metallographically to be important in aligning the cavities.

- The thickness of the inner cylinder is $0.75 \mathrm{~mm}$ to reflect the observed cavitation, and to remain consistent with the thin wall predictions and metallography.

- The MSRC for the outer cylinder is chosen as the MPS at the skeletal point, the same as that used for the prediction of argon pressurised tubes.

The creep curve predictions are shown in figure 6.15, and give rather good fits to the experimental data. There is a tendency to overestimate slightly the rupture time, similar to the thin wall predictions as shown in the rupture plot (figure 6.16).

The main problem with the thick wall model is that it does not necessarily predict the same behaviour as a single wall model when CDM parameters for air are entered into both the inner and outer cylinders. In a single wall model, damage is considered to be homogenous across the tube wall, its value being calculated from the MSRC. Thus, no stress redistribution occurs 


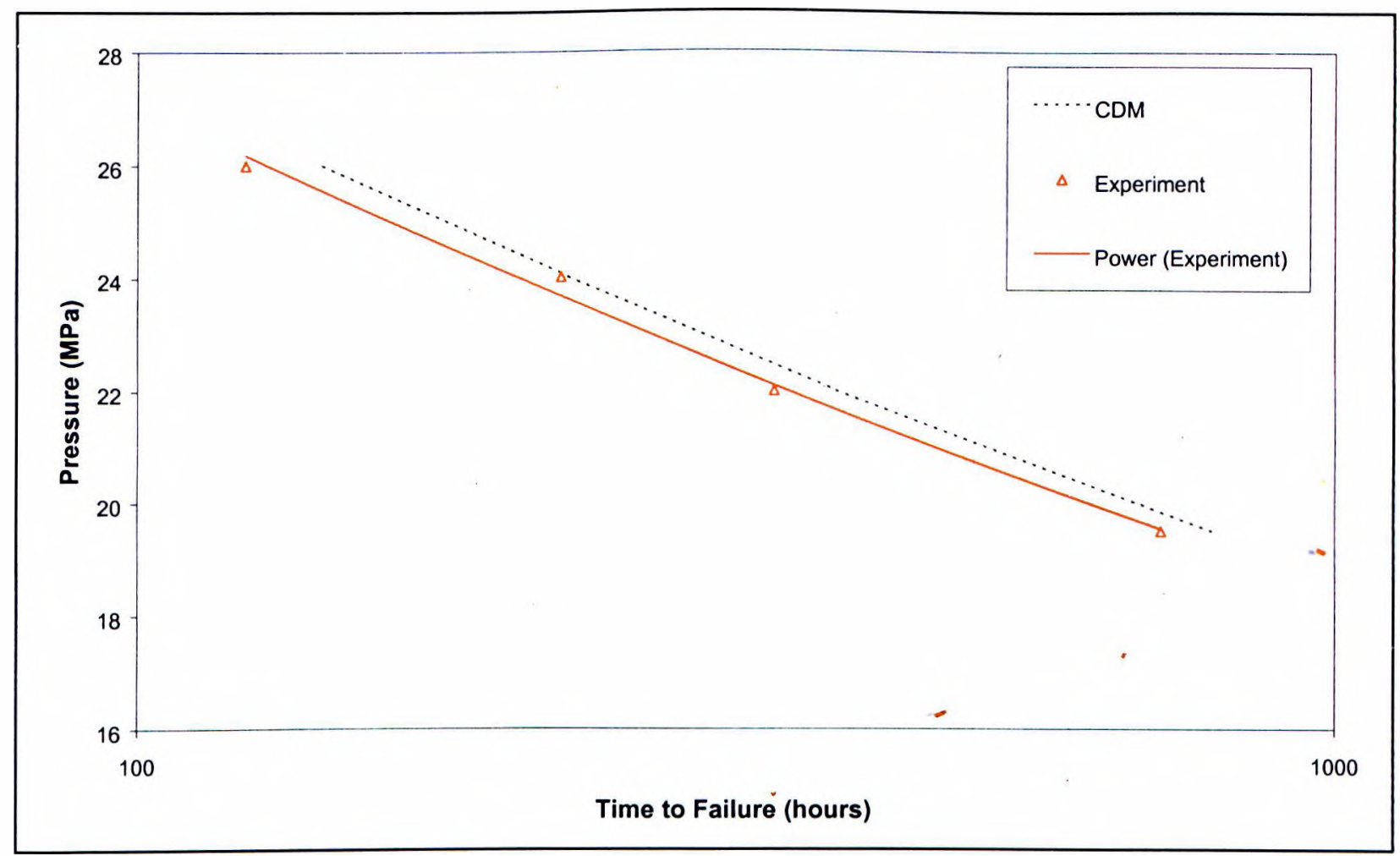

Figure 6.16: CDM predicted pressure-rupture plot for hydrogen pressurised tubes, using thick wall assumptions in the concentric cylinder model.

(excluding the redistribution from elastic to steady state creep situations). With the concentric cylinders two MSRC are used, one for the outer and one for the inner cylinder. Unless these are the same (i.e. they are both taken at the interface between the cylinders and they both have the same $\alpha$ value), then the damage will be different in each cylinder and stress redistribution will occur. The only way therefore that the single wall and concentric cylinder approach will be the same is if the MSRC for the inner cylinder, the outer cylinder and the single wall tube are all taken at the same position corresponding to the interface between the cylinders. Unfortunately, in the current work, choosing this position does not result in good predictions of the experimental data. 


\subsection{Damage Parameter - Metallography Relations}

This section of the modelling chapter aims to find some relationship between the damage parameter, $\omega$, used in the CDM equations, and the actual physical damage occurring in the hydrogen exposed creep specimens. The discussions below will concentrate on uniaxial test specimens.

\section{Theory and Assumptions}

It is assumed that the damage in hydrogen exposed creep specimens is a combination of the damage which would happen normally during creep without hydrogen, and the additional damage which arises due to hydrogen attack. The normal or 'ductile' damage, which occurs without hydroger, is manifested through a combination of mechanisms such as carbide coarsening and local necking. They are arbitrarily summed together in the CDM $\omega$ parameter when fitting the creep curves. The additional damage due to hydrogen attack is caused by grain boundary cavitation, and it is assumed that the CDM omega parameter for hydrogen fitted creep curves is the sum of the part played by normal 'ductile' damage mechanisms, and the part played by cavitation (eqn. 6.14). Surface decarburisation is neglected in this analysis due to its relatively small size compared with the specimen.

$$
\omega_{\mathrm{CDM} \mathrm{H}} \text { curve fit }=\omega_{\text {ductile }}+\omega_{\mathrm{H}_{2}}
$$

\section{Techniques for Finding the Ductile and Hydrogen Damage Proportions}

The following paragraphs describe the techniques used to determine the separate ductile and hydrogen damage parts of the omega parameter. Due to time restrictions, it was not possible to perform interrupted creep tests in hydrogen within the work of this thesis. Therefore, the only record of damage in the material which can be related to the omega parameter is that at failure. The evolution of damage is therefore not discussed in detail.

\section{Determining the Ductile Damage in Hydrogen Exposed Creep Specimens.}

It is assumed that the amount of damage which occurs in a hydrogen exposed creep specimen due to ductile damage mechanisms alone $\left(\omega_{\text {ductile }}\right)$ is approximated by that which would have occurred in a creep specimen tested in air at the same stress for the same time as the corresponding hydrogen creep test. This can be determined easily from the CDM curve fitting model for creep in air by inputting the hydrogen test stresses and their lifetimes. The lifetimes 
used are not the experimental hydrogen test lifetimes but rather the CDM hydrogen curve fit lifetimes. These were chosen because the object of the current work is to relate the model to the damage in the material (the CDM fitted lifetimes are similar to the experimental data and a parameter study showed that in this case it made very little difference whether the experimental data or the CDM fitted values were used).

The ductile damage determined using the above method cannot be directly applied to the total damage occurring in hydrogen exposed specimens (eqn. 6.14). The problem arises because the critical omega parameters at failure are different in the air and hydrogen curve fits $\left(\omega_{\mathrm{fair}}=0.62\right.$; $\omega_{\mathrm{f}_{2}}=0.85$ [see table 6.1]). To overcome this, the omega values for ductile and hydrogen damage must be normalised with respect to the critical omega values at failure. For example, dividing the ductile damage $\omega_{\text {ductile }}$ determined above, by 0.62 , would give the proportion of ductile damage to failure at the given time.

Table 6.2 shows the calculated ductile omega parameter determined by the above methods. The rightmost column shows the normalised value which represents the proportion of the total damage in the hydrogen exposed test specimens which was caused by ductile damage mechanisms, i.e. $\omega_{\text {ductile }}$ in equation 6.14. For example, a creep specimen tested in air with a stress of $104.9 \mathrm{MPa}$ for 225 hours would have been damaged to $68 \%$ of its critical failure damage level. The same conditions led to failure in a hydrogen environment. Thus, it is assumed that, at failure, the specimen was damaged $68 \%$ by ductile mechanisms, with the remaining $32 \%$ being caused by hydrogen attack cavitation. The following paragraphs will discuss a technique which aims to relate this predicted hydrogen damage to metallographic analysis of the test specimens. It is interesting to note from table 6.2 that the proportion of damage due to ductile mechanisms decreases with increasing test time. This reflects the increasing amounts of hydrogen attack seen in the creep specimens as described in section 5.3.

\begin{tabular}{|c|c|c|c|}
\hline $\begin{array}{c}\text { Stress used for creep } \\
\text { testing in hydrogen } \\
(\mathbf{M P a})\end{array}$ & $\begin{array}{c}\text { CDM fitted failure } \\
\text { time for creep in } \\
\text { hydrogen (hours) }\end{array}$ & $\begin{array}{c}\text { (Ductile) omega values } \\
\text { for the given stress and } \\
\text { time, determined from } \\
\text { CDM curve fits of } \\
\text { creep tests in air }\end{array}$ & $\begin{array}{c}\text { Normalised ductile } \\
\text { omega value w.r.t. } \\
\text { critical omega at } \\
\text { failure in air tests } \\
\left(\omega_{\text {ductilc }}\right)\end{array}$ \\
\hline 104.9 & 225 & 0.42 & 0.68 \\
\hline 94.9 & 367 & 0.36 & 0.58 \\
\hline 86.8 & 573 & 0.33 & 0.53 \\
\hline 83.9 & 666 & 0.31 & 0.50 \\
\hline
\end{tabular}

Table 6.2: Calculations of the normalised ductile omega parameter for determination of the proportion of damage in hydrogen exposed specimens due to ductile damage mechanisms. 


\section{Metallographic Determination of the Hydrogen Attack Damage}

One commonly used method for determining a damage parameter from metallographic evidence of cavitation is the 'A' parameter [Shamma.' $87 \mathrm{~b}$ ], [Furtad.'97]. The ' $A$ ' parameter is basically a representation of the number fraction of cavitated grain boundary facets in the material. For the current work, the rules determined by Shammas were followed for the determination of the ' $A$ ' parameter; listed below and illustrated in figure 6.17 [Shamma.' $87 \mathrm{~b}$ ]]:

1. An intersected grain boundary is only observed between the first triple point on either side of the intersection. If the boundary extends beyond the field of view then the point at which it leaves is treated as the triple point.

2. A grain boundary is classified as DAMAGED if it contains one or more cavities (or microcracking) along its observable length including cavities centred on the triple point itself, otherwise the boundary is UNDAMAGED. If in doubt as to whether a feature is a cavity or not it is disregarded.

3. Multiple intersections with the same boundary are each counted and are classified with the damage state of the whole boundary.

4. Intersections with triple points count as one boundary intersection. The classification of DAMAGED or UNDAMAGED is determined by a 'majority vote' of damage states of the three jointed boundaries.

The ' $\mathrm{A}$ ' parameter is calculated as the ratio of damaged grain boundaries to total grain boundaries intersected by a line drawn parallel to the stress direction (figure 6.17).

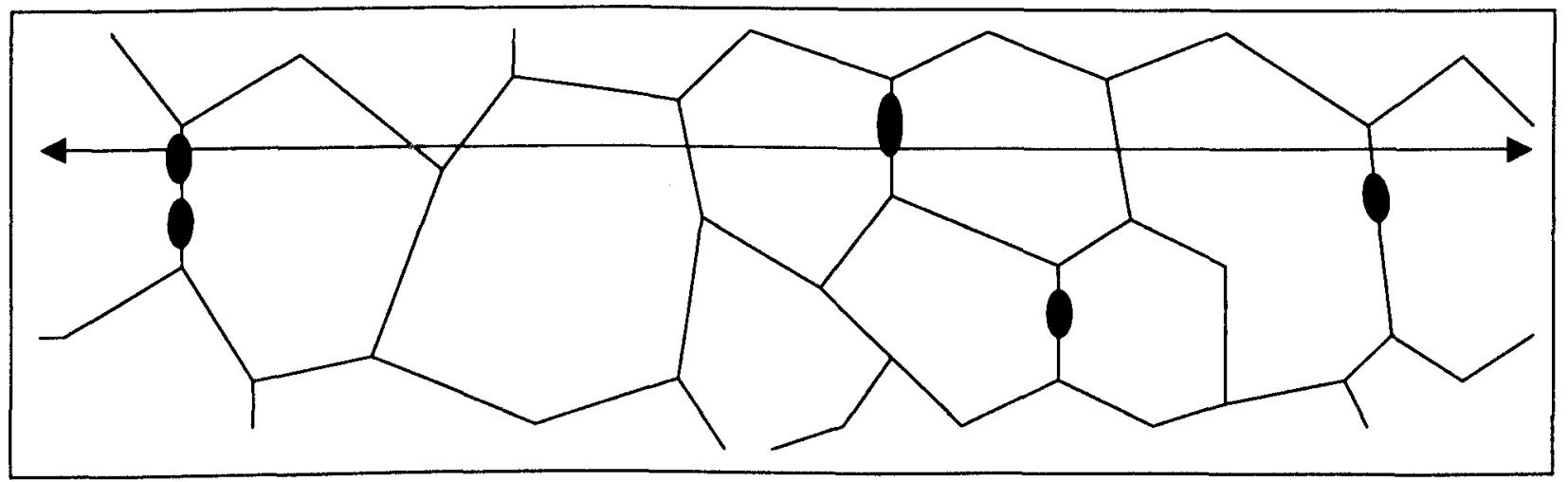

Figure 6.17: Illustration demonstrating measurement of the 'A' parameter. The double headed arrow is aligned parallel to the stress. It crosses 7 grain boundaries, three of which are cavitated, giving an 'A' parameter of $3 / 7$ or about 0.43 . 
Measurements of the 'A' parameter were made at many positions along the specimen gauge length. There did not appear to be any significant differences.

Once the ' $A$ ' parameter has been determined, it is then converted into the damage parameter, omega. Some authors [Stamm'93] suggest that stereological considerations should be taken into account when performing this conversion. However, a much simpler technique is to simply equate the 'A' parameter with the omega parameter [Shamma.'87b]. Since no cavitation was observed in creep tests in air (within the given conditions) it is assumed that all of the cavitation is due to the influence of hydrogen, and the hydrogen attack omega value, $\omega_{\mathrm{H}_{2}}$, can be taken as equal to the ' $A$ ' parameter. However, the 'A' parameter is normally related to omega when omega is taken as equal to 1 at failure (as is normally the case when using the CDM equations). Thus the omega values must be normalised, similar to the ductile case, by the critical damage at failure for the hydrogen exposed specimens. This means dividing the measured values by 0.85 . The results of the ' $A$ ' parameter measurements are shown in table 6.3.

\begin{tabular}{|c|c|c|}
\hline Hydrogen test stress (MPa) & $\begin{array}{c}\text { Average measured 'A' } \\
\text { parameter at failure }\end{array}$ & $\begin{array}{c}\text { Normalised 'A' parameter w.r.t. } \\
\text { the critical omega at failure. } \\
\left(\omega_{\mathrm{l}_{2}}\right)\end{array}$ \\
\hline 104.9 & 0.37 & 0.45 \\
\hline 94.9 & 0.41 & 0.49 \\
\hline 86.8 & 0.43 & 0.52 \\
\hline 83.9 & 0.45 & 0.54 \\
\hline
\end{tabular}

Table 6.3: Measured 'A' parameter values from creep tests in hydrogen, and conversion to the normalised hydrogen attack damage omega parameter for determination of the proportion of hydrogen attack damage at failure.

The proportion of damage caused by hydrogen attack can be seen to increase with decreasing test stress or increasing test time. This reflects the increased amounts of hydrogen attack damage observed under longer test times.

The proportion of damage due to hydrogen calculated with the ' $\mathrm{A}$ ' parameter (table 6.3) can be compared with that predicted from calculations of the ductile damage proportion (table 6.2) by subtracting the ductile damage proportion from 1. This comparison has been made in figure 6.18. Under lower stresses the value determined with the ' $A$ ' parameter, and that predicted using the CDM equations are quite similar. Under higher stresses, there is some discrepancy between the values. The reason for this could be in the way that the ' $A$ ' parameter is calculated. For example, figure 6.19 shows two grain boundaries which are cavitated to different extents. These represent the types of damage seen under high and low stress tests (section 5.3). The low stress situation is clearly more damaged, yet the ' $A$ ' parameter would treat both of these grain 


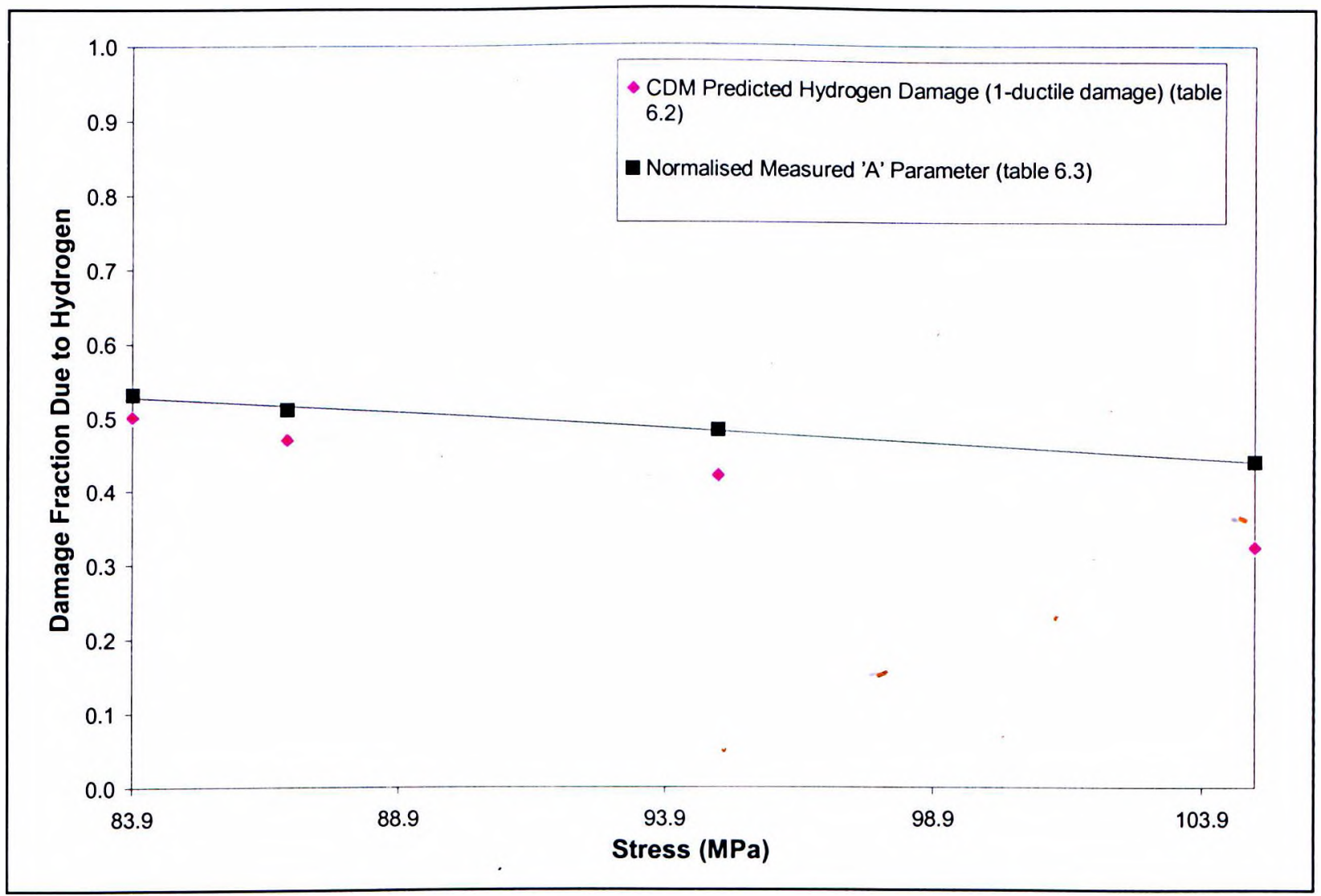

Figure 6.18: Comparison between the hydrogen damage fraction at failure in uniaxial test specimens calculated using the 'A' parameter and predicted using the CDM curve fitting equations.

boundaries in the same way. This could explain why the ' $A$ ' parameter overestimates the proportion of hydrogen attack damage in high stress tests.

\section{$\underline{\text { Summary }}$}

The simple use of the ' $A$ ' parameter here gives a reasonable impression of the level of hydrogen attack damage in the material at failure, particularly under lower stress/longer term tests. For more accurate predictions, it may be necessary to include a measure of the extent of cavitation on a single grain boundary facet rather than just the number of cavitated grain boundary facets. The technique proposed here should be validated at all stages of the creep test, and not just at failure. 


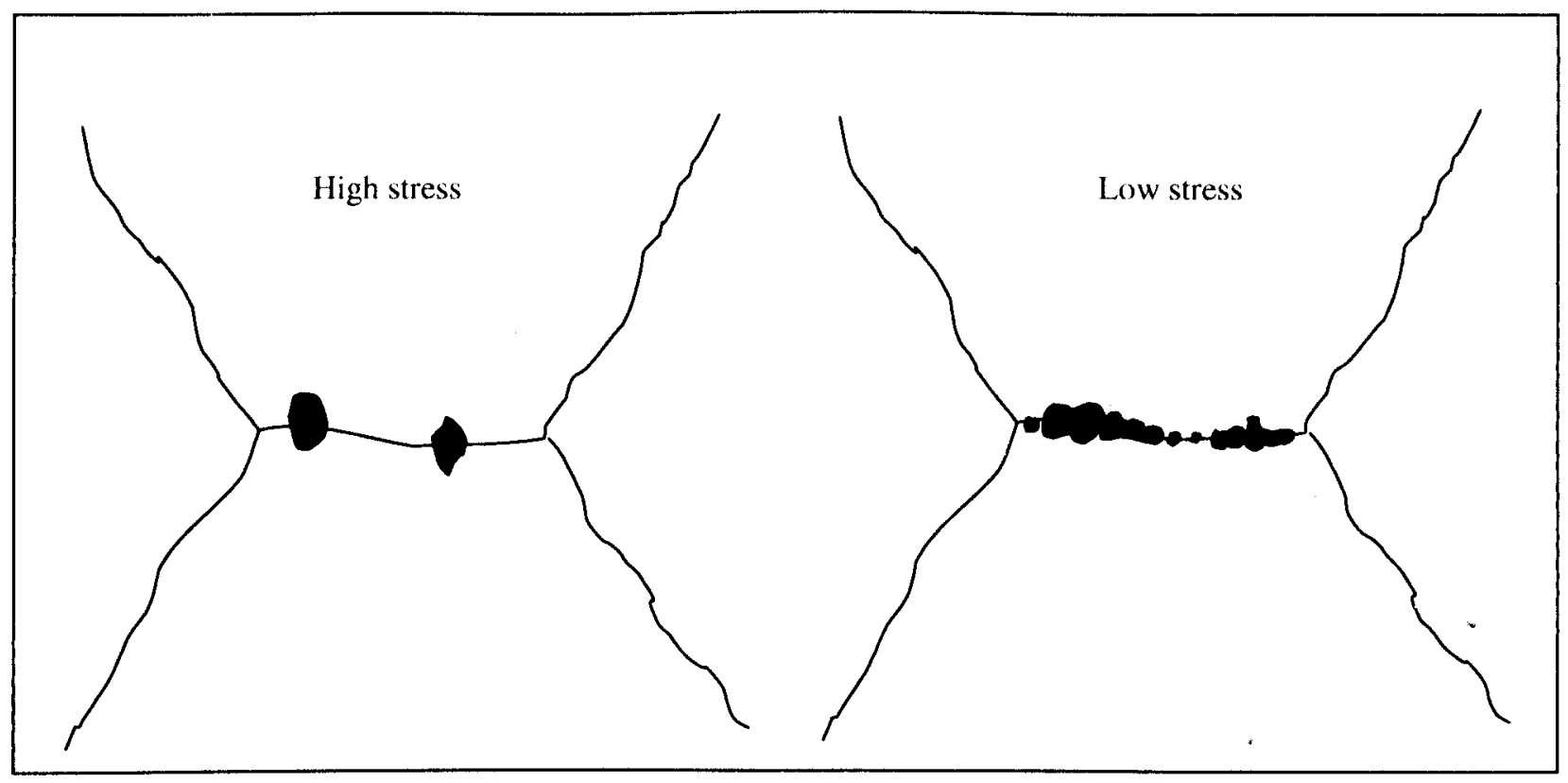

Figure 6.19: Illustration to show the difference in grain boundary cavitation in high and low stress tests. Note that the low stress test shows a grain boundary which can support virtually no stress, whilst the high stress situation shows a grain boundary which can still transmit stress. The 'A' parameter treats both the same. 


\section{GENERAL DISCUSSION}

The following chapter aims to bring together the results presented in chapter 5 in order to gain some useful information from them. Initially, the cavitation observed in creep tests in hydrogen will be discussed in terms of the driving forces for cavity growth, namely the external stress and the internal methane pressure. Results from chapter 6 concerning modelling will be incorporated to attempt to determine the effect of the multiaxial stressing in the tubes tests. A brief discussion then follows on some of the observations of surface decarburisation mentioned in chapter 5 . 


\subsection{The Effect of Combined Creep Stress and Hydrogen Attack on Cavitation}

The main characteristic of hydrogen attack damage is grain boundary cavitation. Cavitation is also a form of damage often associated with creep. In fact, most models of hydrogen attack cavitation in the literature use equations originally derived for the case of creep cavitation, modified to account for an internal methane pressure rather than, or together with, an externally applied remote stress [Shewmo.'76], [Sagues'78], [Shih'82], [Sundar.'81], [Shewmo.'87], [VdBurg'96]. Furthermore, it has been suggested that the cavity nuclei are the same for both hydrogen attack and creep processes [Lopez'83]. Hydrogen attack in the absence of applied stress is an internal pressure driven cavity growth phenomena, and creep damage (without hydrogen) is an externally applied stress driven cavity growth phenomena. The combination of the two can increase the driving force for cavity growth. The effect of applied stress on hydrogen attack and, similarly, the effect of hydrogen attack on creep are both related directly to the influence of internal pressure and external stresses on cavity nucleation and growth. The results presented in section 5 will now be discussed in those terms.

\section{Cavitation Under External Stress Only and Internal Methane Pressure Only}

\section{Cavitation Under External Stress Only (uniaxial creep in air-section 5.2)}

Within the conditions studied, no grain boundary cavitation was observed in the creep specimens tested in air. It is expected that under lower stress/longer testing times, cavitation would become obvious in this steel and would dominate fracture [Church'92], [Fields'80], whereas under the short term conditions studied, failure was dominated by deformation and ductile failure mechanisms.

\section{Cavitation Under Internal Pressure Only (autoclave exposures section 5.1)}

Within the conditions studied, no cavitation was observed in the autoclave hydrogen exposed material. However, it is expected that longer exposure times would reveal cavities, as has been observed elsewhere for similar steels [Manola.'98].

\section{The Effect of Time on Limiting Cavity Observations}

It was determined for the examination techniques used in the current work, that it was not possible to positively identify a metallographic feature as a cavity unless its diameter was greater than approximately $0.25 \mu \mathrm{m}$. Below this size, it became difficult to distinguish between 
inclusions, carbide shadows and etching relief (see section 5.1 for examples of observation artefacts). The initial diameter considered for nuclei has been stated for quenched and tempered 2.25Cr-1Mo steel as being between $0.01 \mu \mathrm{m}$ and $0.18 \mu \mathrm{m}$ [Shewmo.' 83 ], with a critical size for growth estimated at $0.02 \mu \mathrm{m}$ [Lopez'83]. The cavities must therefore grow substantially before they can be clearly seen. Thus, the lack of observed cavities for both pure internal methane pressure and pure external stress does not necessarily mean that there is no cavitation, but could merely indicate that under the given conditions the exposure time was not long enough to make them visible.

\section{Cavitation Under Combined Stress and Internal Methane Pressure}

\section{The Effect of Uniaxial Stress on Cavitation (for a given methane pressure)}

Cavitation was clearly seen in the gauge lengths of the uniaxial creep specimens tested in a hydrogen environment under similar exposure conditions to the autoclave tests. Therefore, the combined effect of stress and internal methane pressure was to produce cavities under conditions where neither the stress nor the methane pressure alone was able, i.e. the application of stress to a material undergoing hydrogen attack can greatly accelerate the cavity growth rate. Similarly, creep strength and ductility in a hydrogen environment are reduced due to the accelerated growth of cavities.

The specimen shoulders, where the stress (and strain) is much lower, showed no observable cavitation. The time of exposure and the methane pressure in the shoulders and the gauge length are the same. Thus, it appears that the reason for increased cavitation in the gauge length was the higher stress, promoting greater cavity growth rates for a given methane pressure. The internal methane pressure for uniaxial test conditions (hydrogen pressure 22MPa) was estimated as 106.2MPa (Appendix E).

\section{The Effect of Multiaxial Stress on Cavitation}

The tubular specimens pressurised with hydrogen also produced cavitation, whereas control testing with argon had not. Since the stress state in the tube is multiaxial, it should be possible to determine if the increase in the stress triaxiality had any effect on the cavity growth rate. Analysis of the tubular behaviour is complicated because of the non uniform cavity distribution observed and the hydrogen gradient across the tube wall (and hence methane pressure gradient $\sim$ discussed later). In addition, it is expected that some stress redistribution occurs due to the non uniform damage. 
A comparison between the effect of uniaxial and multiaxial stress can be made by comparing the stresses in the two types of test and correlating this with the cavity observations. This has been done by comparing the maximum principal stress because metallographic evidence shows cavities growing preferentially on those grain boundaries at $90^{\circ}$ to the MPS. Due to the cavity gradient in the tubes (high near the inner wall - low near the outer wall), it is necessary to define a depth in the tube wall where the cavitation can be compared with the uniaxial case. This depth has been taken as $0.37 \mathrm{~mm}$ since in this area cavitation was at, or near, a maximum severity in most of the tubes studied (surface decarburisation reduces cavitation immediately next to the inner wall). It is assumed that the MPS at this point can be calculated accurately from the thick wall concentric tube model described in section 6. To account for any stress redistribution and stress increases due to strain, the weighted average stress operating throughout the lifetime was calculated. This average stress is plotted against the exposure time in figure 7.1 for each of the tubes studied. The number next to each of the points represents the estimated methane pressure at that position within the tube wall (Appendix E). Also shown in the figure are the average uniaxial gauge length stresses (taking into account stress increase

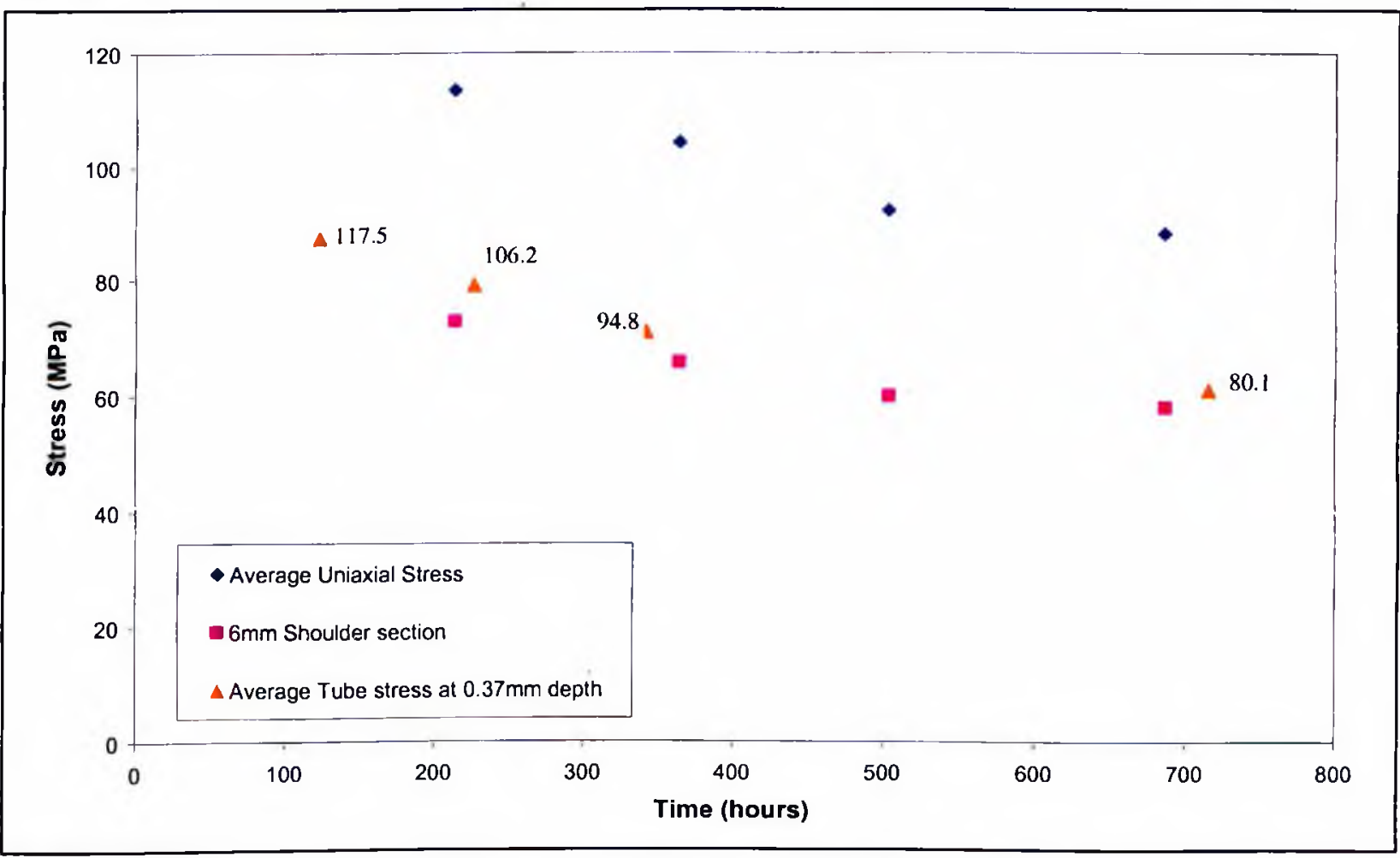

Figure 7.1: Average stresses plotted vs. time for uniaxial test stresses, uniaxial specimen shoulder stresses, and tubular MPS stresses at $0.37 \mathrm{~mm}$ wall depth. Numbers represent the estimated methane pressures at the given wall depth. 
with strain) and the estimated stresses operating at the thinnest part of the uniaxial specimen shoulders (ignoring potential stress concentration effects).

The exposure time for three of the tubes (labelled 106.2, 98.4, 80.1) are very similar to three of the uniaxial tests. This allows the effect of the multiaxial stress to be compared without complications due to different exposure times. The calculated average MPS in these tubes at the position of maximum cavitation is somewhat lower than the average uniaxial stresses for similar exposure times. In fact, the average MPS in the tubes corresponds quite well to the estimated stress in the thinnest part of the uniaxial specimen shoulders. No cavitation was observed in the uniaxial shoulders, and it was stated in section 5.3 that the cavity situation in the tubes appeared locally as severe, if not more severe, than in the gauge length of the uniaxial specimens. In addition, the methane pressures are generally lower in the tubes at the position of maximum cavitation than in the uniaxial specimens (see later for discussion of the effect of methane pressure). This strongly suggests that, for a given MPS, the cavities were able to grow faster in the tubes than in uniaxial specimens. The MPS is therefore important in cavity nucleation (due to the alignment of cavities at $90^{\circ}$ to it), but it alone does not control the cavitation. It is then reasonable to suggest, without going into details of the cavity growth mechanisms, that the higher stress triaxiality found in the tubes accelerated the cavitation. This is similar to the findings of Cane who studied creep cavitation (without hydrogen) in $2.25 \mathrm{Cr}$ 1Mo steel [Cane'81].

It should be noted that the tube which failed in 123 hours did not show cavitation damage, despite having lower creep strength than a tube pressurised with argon at the same pressure. This is probably due to the short time of the test which did not allow the cavities to grow to sizes where they were observable, yet may have allowed them to have some influence on the creep properties. The differences in the creep properties may also be due partially to experimental scatter.

\section{The Effect of Hydrogen/Methane Pressure on Cavitation}

As mentioned above, there is a hydrogen concentration gradient which builds up across the tube wall. To a first approximation, the effective pressure at the inner tube wall is equal to the applied test pressure, and at the outer wall the effective pressure is 0 . This will cause the methane pressure to drop across the tube wall as well (see Appendix E for methane pressures).

The tubular specimens showed a gradient of cavitation, being higher near the inner wall (excluding surface decarburisation) and dropping off towards the outer wall. Cavities were 
scarcely seen at the middle of the tube wall thickness. This cavitation gradient could be potentially explained by either the stress distribution across the tube wall, or by the hydrogen concentration gradient. The MPS is shown to be higher towards the outer wall under steady state creep conditions [Bailey'51] and, according to the model (section 6), this stress will become even higher away from the inner wall due to stress redistribution. This stress gradient is the opposite to the cavitation gradient, and given cavity nucleation appears to be mostly controlled by the MPS and that the effect of stress has been demonstrated above, it seems unlikely that the stress distribution in the tubes is the reason for the observed cavity distribution.

The cavity gradient in the tuhe is then most probably determined by the hydrogen concentration gradient, showing that the higher the hydrogen concentration (and hence higher hydrogen and methane pressures), the higher the cavity growth rate. More quantitative measurements were beyond the scope of this work. The hydrogen attack in the tubes is determined by the local conditions of methane pressure and stress.

\section{Changes in Cavitation Observations for Differing Test Duration}

There was a difference noted in the cavitation observed in high stress-short term tests and low stress-long term tests. These differences were most apparent in uniaxial creep due to the better homogeneity of cavitation, but were also noticeable in the tubes. Under high stress-short term testing, the cavities tended to be large but more spaced out on the grain boundary facets, whereas the longer term tests showed smaller, more closely spaced cavities on the boundary facets, leading to almost complete decohesion of the grains and consequent brittle failure. The following paragraphs present possible explanations of the observed behaviour.

It has been proposed in the literature that hydrogen attack in $2.25 \mathrm{Cr}-1 \mathrm{Mo}$ steel nucleates from pre-existing sites in a given steel. However, in creep, it is often assumed that nucleation is continuous, caused by grain boundary sliding events. At high stresses, the sliding rate decreases relative to the macroscopic creep strain rate [Cane'79]. Thus, the high stress tests will have less new nuclei than lower stress tests for a given creep strain. This may explain why there appeared to be more cavities in the lower stress tests (and resulting lower ductility). The larger size of the cavities in the high stress tests may well be caused by the higher strains at failure which would open up the cavities further, and also by the increased diffusional cavity growth mechanisms which would be expected under such conditions. Conversely, the lower stress tests would nucleate larger numbers of cavities with slower growth rates resulting in the observations of strings of small, closely spaced cavities. 
Similar observations were made for autoclave hydrogen exposures of a carbon steel and a low carbon steel in the absence of external stress (Figure 7.2). The carbon steel develops a very high methane pressure which causes very rapid cavity growth initiating almost exclusively from preexisting nucleation sites situated at the poorly cohered manganese sulphide-matrix interface. These cavities grow very rapidly until the steel is totally decarburised resulting in several areas with very large cavities. In contrast, the lower methane pressure in a low carbon steel causes relatively slow cavity growth rates on grain boundaries. In these steels it has been suggested that nucleation is continuous [Vitove.'64], being caused by the tensile stress which is set up on the grain boundary as a neighbouring cavity grows. Thus, in this steel, strings of small cavities are observed on a few grain boundaries before the steel becomes decarburised and the reaction stops.

Hydrogen attack models which consider only pre-existing nuclei may have problems to predict the types of behaviour described here.
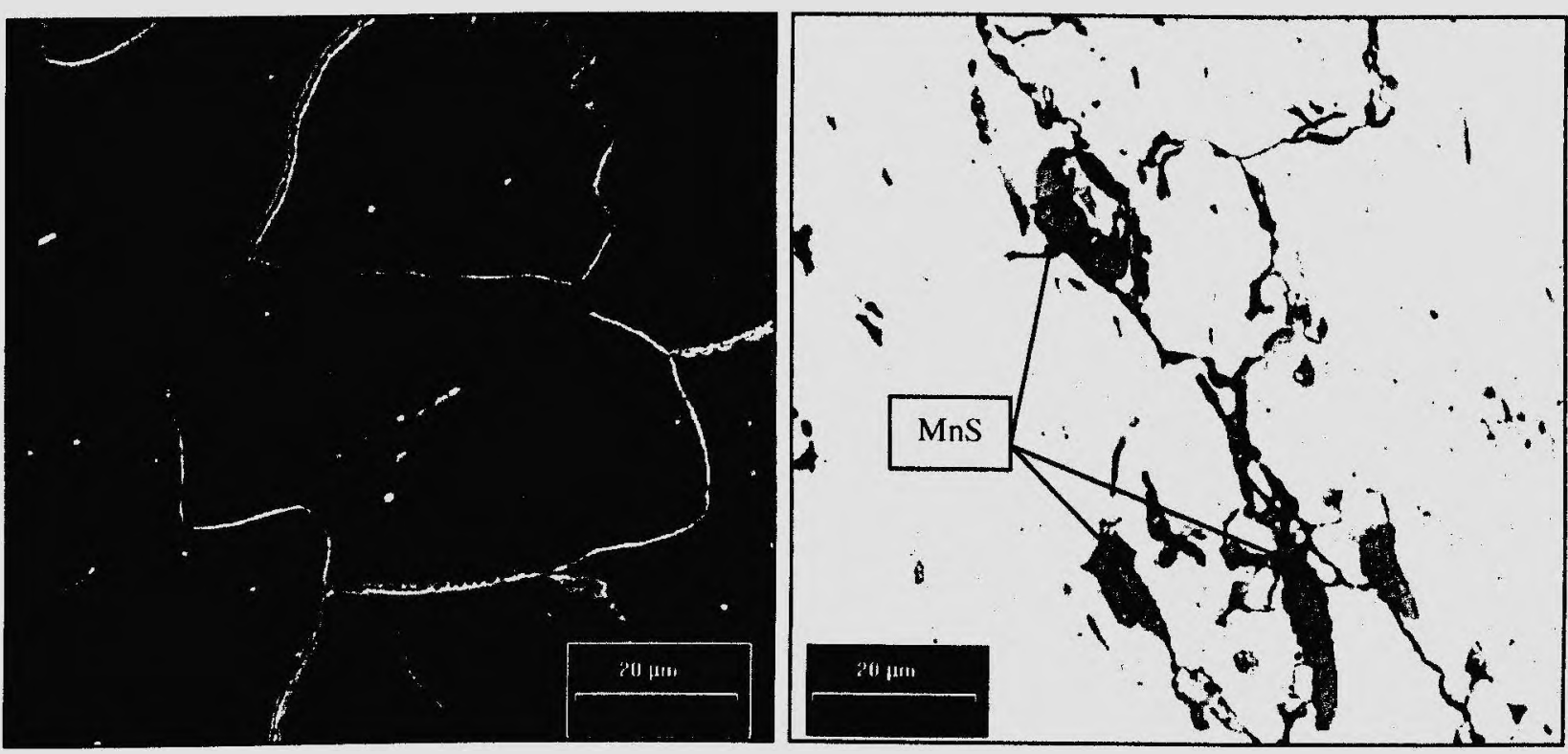

Figure 7.2: Low carbon steel (left) and plain carbon steel (right) exposed in an autoclave for 395 hours under 200bar hydrogen at $600^{\circ} \mathrm{C}$. Note the difference in morphology of attack between the specimens, related to the different methane pressures. The carbon steel micrograph was taken with back-scattering electrons to show up the MnS inclusions (dark grey). 


\subsection{The Importance of Surface Decarburisation}

Surface decarburisation was observed in all of the tests performed in the work of this thesis. The following discussion aims to show how the decarburised zone can be used to learn about the hydrogen attack phenomena, and how it can effect the results of laboratory tests.

\section{Surface Decarburisation as a Tool for Understanding Hydrogen Attack.}

\section{General Observations}

During surface decarburisation, the methane reaction occurs on the steel surface between dissolved carbon and the hydrogen environment. The methane formed on the surface is free to escape so, unlike internal cavities, no equilibrium methane pressure builds up. The driving force for the reaction is therefore continuous, and a carbon depleted region forms at the steel surface. Equilibrium dictates that the carbides in this region dissolve to replace the lost matrix carbon. The newly released carbon can then diffuse to the surface where it reacts with hydrogen, and a continuous decarburisation process occurs. Importantly, the decarburised zone shows that carbides can dissolve readily, and that the dissolved carbon can diffuse over relatively large distances to reach the steel surface (up to about $200 \mu \mathrm{m}$ observed in the current work). This suggests that hydrogen does not have to react directly with the carbides in the steel to form methane in internal voids, as has been sometimes assumed in the literature. It is further suggested here, that the absence of surface decarburisation means the absence of the methane reaction, and therefore (theoretically) the absence of hydrogen attack.

\section{Surface Decarburisation and Carbide Stability}

It was shown in section 5.1 how the decarburised zone could be used to study the carbide stability in a given steel, and a depth profiling technique was suggested for ranking the carbide stabilities. It was found in the current work that the $\mathrm{M}_{0} \mathrm{C}$ type was the most stable carbide, which is also usually considered as stable with respect to tempering and ageing. This stability should correspond with lower carbon activity, and hence lower methane pressures and lower cavity growth rates.

The depth of decarburisation (for given exposure conditions) reflects the kinetics and thermodynamics of the methane reaction, crucial for hydrogen attack. Hence, the depth of decarburisation may potentially be used as an indicator of the methane reaction rate and, in conjunction with other information, the hydrogen attack cavitation susceptibility. 


\section{The Effect of Surface Decarburisation in Laboratory Creep Tests}

\section{The Effect of Decarburisation on Uniaxial Creep Testing}

For a given temperature, hydrogen pressure and steel, the depth of decarburisation depends on mainly on time. Thus, long term creep tests are more susceptible to the effects of decarburisation than shorter ones. It is assumed that the decarburised zone has a lower creep strength than the base material due to the lower carbon content [Woodhe.'65]. If the zone becomes too large relative to the specimen diameter, it will effect the overall creep strength of the specimen. In the current work, it is believed that the depth of decarburisation in the longest term tests did not significantly effect the creep strength, especially during the early parts of the test when the decarburised zone is still growing. However, care should be taken when performing longer term or higher temperature tests to ensure that the specimen diameter stays large relative to the zone.

\section{The Effect of Decarburisation on Internally Pressurised Tube Testing}

Under long term testing, microcracks in the internally pressurised tube tests began to initiate from within the tube wall instead of from the inner surface. This was caused by the lower cavitation and increased ductility of the decarburised zone. Whilst it is not expected that this greatly affected the test results, it could have more dramatic consequences under longer term test conditions. In addition, should this mechanism of damage occur in industrial pressure vessels, it may effect the choice of NDE method used to detect cracks due to their sub-surface nature. 


\section{SUMMARY AND CONCLUSIONS}

\subsection{Main Conclusions}

The following points list the major conclusions from the work of this thesis. It should be noted that these conclusions apply to the testing of the given steel under the given exposure conditions. Further work, expanding on the results presented in this thesis, may allow the conclusions to be applied more generally to the case of hydrogen attack and creep.

\section{Experimental Testing}

- Full, accurate creep curves can be determined safely using the specifically designed hydrogen creep rigs developed during the present work. Stable hydrogen pressures of up to $220 \mathrm{bar}$, temperatures over $600^{\circ} \mathrm{C}$, and uniaxial loads of up to $10 \mathrm{kN}$ are possible in the rigs, allowing for the detailed study of the effect of hydrogen attack on creep behaviour.

- It is believed that the first hoop strain creep curves from hydrogen pressurised tubes have been determined accurately and safely using the internally pressurised tube testing facility developed during the thesis. A unique 12-gauge capacitive displacement transducer allows the maximum hoop strain to be measured more accurately than conventional methods. Stable pressures up to 270 bar hydrogen at a temperature of $600^{\circ} \mathrm{C}$ have been safely used, with accurate strain readings up to $35 \%$ hoop strain. The rig allows for the study of hydrogen attack in conditions which simulate closer the industrial situation than uniaxial tests alone.

\section{Observations on Combined Creep - Hydrogen Attack Interaction}

- Creep rupture lives and ductility are decreased in the presence of high pressure hydrogen in both uniaxial specimens and internally pressurised tubes, relative to tests in inert environments. The effect is greater under longer test duration. The minimum creep rate is increased in hydrogen. The deterioration of the creep properties is caused by the accelerated growth of methane-filled grain boundary cavities.

- Cavities grow preferentially on those grain boundaries oriented perpendicular to the applied uniaxial stress, demonstrating the importance of the stress. In internally pressurised tube tests, the cavities show some preferential growth on those boundaries perpendicular to the maximum principal stress. This confirms the importance of the stress in accelerating the 
cavity growth rate and suggests that the MPS is dominant in determining the cavity nucleation in multiaxial conditions.

- Tubular components fail from localised cavity coalescence leading to microcrack initiation at the inner wall of the tube. One crack propagates across the tube wall, resulting in failure. Tubes tested with argon gas failed by ductile thinning, initiating from the outer tube wall.

- In creep tests, there is a competition between ductile damage mechanisms and brittle intergranular cavitation. Under high stress tests, the ductile damage mechanisms are dominant, resulting in higher ductility and lower cavity counts, whereas cavitation dominates under lower stress conditions.

- Cavitation is more uniform in uniaxial specimens than in tubes. For similar lifetimes but lower principal stresses, the cavitation may appear on average to be less in the tubes than in the uniaxial specimens. However, locally the cavitation damage in the tubes may be as severe, or worse in the tubes. Thus, in tubular components the local conditions control the failure.

\section{The Effect of Stress, Hydrogen Pressure and Time on Cavitation}

- The application of a uniaxial tensile stress to a steel under hydrogen attack conditions greatly accelerates the growth rate of methane-filled cavities, confirming theory and limited experimental evidence from the literature.

- The accelerating effect of stress on the methane-filled cavity growth rate appears to be greater under the higher stress triaxiality found in internally pressurised tubes. Whilst the MPS appears to influence cavity nucleation, it does not appear to control the later growth of the cavities.

- The effect of exposure time on cavitation is to allow the cavities to grow to sizes at which they can be observed and which can affect the properties of the material.

- The effect of hydrogen concentration/pressure is shown by the cavitation gradient which exists across the wall of the internally pressurised tubes. Higher hydrogen pressures lead to faster cavity growth rates.

\section{Surface Decarburisation}

- Cavitation is hindered in the decarburised zone. 
- Under longer term creep testing at high temperatures in hydrogen, the depth of the surface decarburised zone may affect the overall creep strength of the test specimens.

- Surface decarburisation in internally pressurised tubes caused the microcracks to initiate away from the inner surface. Should this mechanism occur in industrial situations, then it may effect the choice of NDE required to detect such cracks due to their sub-surface nature.

- Hydrogen does not have to react directly with carbides in the steel to produce methane, and will readily react with dissolved carbon, as demonstrated by the decarburised zone.

- The most stable carbide against hydrogen attack in the steel studied was $\mathrm{M}_{6} \mathrm{C}$. The decarburised zone can be used to determine this.

- The depth of decarburisation can potentially be used to give relative information on the hydrogen attack resistance of steels.

\section{Creep Modelling}

- A strain hardening primary creep form of the CDM equations has been successfully developed for the fitting of uniaxial creep curves obtained under constant load conditions. Good curve fits for both creep in air and hydrogen have been determined.

- The CDM model has been successfully applied to the prediction of the creep behaviour of both argon and hydrogen pressurised tubes. Hydrogen pressurised tubes were modelled by assuming a concentric cylinder model.

- The damage parameter, omega, from the uniaxial CDM model can be related to the 'A' parameter for cavitation. The relation works best under longer term testing conditions when cavitation is more dominant.

- The minimum creep rates in hydrogen pressurised tubes can be correlated with uniaxial creep data in hydrogen by using the Von Mises skeletal point stress. The rupture times are correlated by taking the mean diameter hoop stress for reference. These are the same reference stresses used to correlate argon pressurised tubes with uniaxial creep data in air.

- The reference stress techniques and the CDM model may be potentially used to extrapolate laboratory data to real industrial components provided that the correct correlation can be found between the high stress, high temperatures used in the laboratory to the less severe industrial conditions. 


\subsection{Suggestions for Further Work}

The current work has shown qualitatively the effect of stress, time and hydrogen pressure on hydrogen attack. A particular temperature was chosen in order to restrict the test matrix. For useful application to the case of hydrogen attack in industrial pressure vessels, more quantitative information is required. This can be achieved by performing a larger testing matrix of various pressures, stresses and temperatures in the testing facilities already developed. The following points are suggested guidelines for performing future studies.

- Allow plenty of time in laboratory studies to ensure cavitation becomes visible. This will permit better comparison between the various tests and may help to show to what extent stress increases cavitation relative to the autoclave style exposures (without stress). Alternatively, different metallographic techniques and higher resolution imaging could be used to study the growth of cavities too small to be seen in the current work. Such work could also be useful for studying the nucleation of cavities which appears to be important in determining the extent of attack under various stresses.

- Make sure specimens are large when performing long term creep tests at high temperature to minimise the effect of surface decarburisation. It is estimated that the $5 \mathrm{~mm}$ gauge diameters used in the current work would probably be too small in tests lasting above about 1000 hours at $600^{\circ} \mathrm{C}$.

- Due to the relatively high ductility of the material used in the current work, the stress increased significantly during testing, making analysis and modelling more complex. For better quantification of the effect of stress, it may be better to use constant stress testing where possible.

- Due to limitations on laboratory test duration (e.g. high costs), higher temperatures than those used in industry were used to accelerate the tests. To confirm the models and to ensure no major mechanistic differences occur, it may be necessary to perform some extra tests at lower temperature. Also, some tests at lower stresses should be studied, being more representative of the industrial situation.

- Minimise the interrelation of parameters, i.e. in the tube tests the stress was increasing with strain, the stress was controlled by the pressure and the rupture time was determined by the stress. In addition, a gradient of hydrogen pressure existed in the material. This makes 
quantitative analysis difficult. Different testing techniques might be considered such as constant stress testing, notched bars and biaxial tension.

- The effect of multiaxial stress in particular should be examined closer. Internal pressure tests with combined end loading, bending, notched bars or biaxial tension for example could be used to determine the effect of varying amounts of stress triaxiality.

- Interrupted tests would be extremely useful for determining the cavity growth rate and the evolution of the damage. This data could be used for verifying models of cavitation, and to test the relationship between the ' $A$ ' parameter and the CDM damage parameter found in the current work. This relation should also be tested under different exposure conditions.

- The full importance of the methane pressure and the effect of carbide stability thereon has still not been demonstrated experimentally. Techniques for,measuring the methane pressure, for example, melt degassing, should be developed.

- The current work studied base material only. This has allowed some general observations to be made and models to be developed. However, the most critical part of most components are welds, which are also known to be more susceptible to hydrogen attack. Using the observations and models developed here as baseline data, it may be useful to extend the work to the study of combined creep and hydrogen attack of welds. Similarly, many industrial pressure vessels contain an inner cladded layer. The effect of this on the hydrogen attack kinetics has not been fully analysed. 


\section{APPENDIX A}

\section{Surface Changes on Internally Pressurised Tubes}

\section{Observations}

All internally pressurised tubes tested in the work of this thesis have shown a matt grey surface finish on their outer wall after testing. This surface finish has been observed for both internal argon, and hydrogen pressure tests. However, some differences exist between the layers formed during argon and hydrogen pressurisation. In the case of hydrogen pressure, the surface tends to have a much lighter colouration than in argon. For argon pressurised tubes, the colour is often nearly black. In addition, the surface tends to spall off in a thin layer under hydrogen, whereas the surface is always strongly adherent to the tube in the case of argon.

Some of the spalled surface from a hydrogen pressurised tube was examined in more detail. It was found that the underside of the layer was a black colour, in contrast to the lighter upper surface. SEM analysis shows that the spalled surface has double layer structure (figure Al). The outer layer has a very porous appearance, whilst the inner is heavily cracked in a regular fashion (figure A2). EDS analysis shows that the outer layer consists of virtually pure iron, whilst the inside also contains a lot of chromium, molybdenum and oxygen. The total thickness of the two layers together is approximately $20 \mu \mathrm{m}$.

\section{$\underline{\text { Discussion }}$}

It has been shown that steels operating under low partial pressures of oxygen may form a
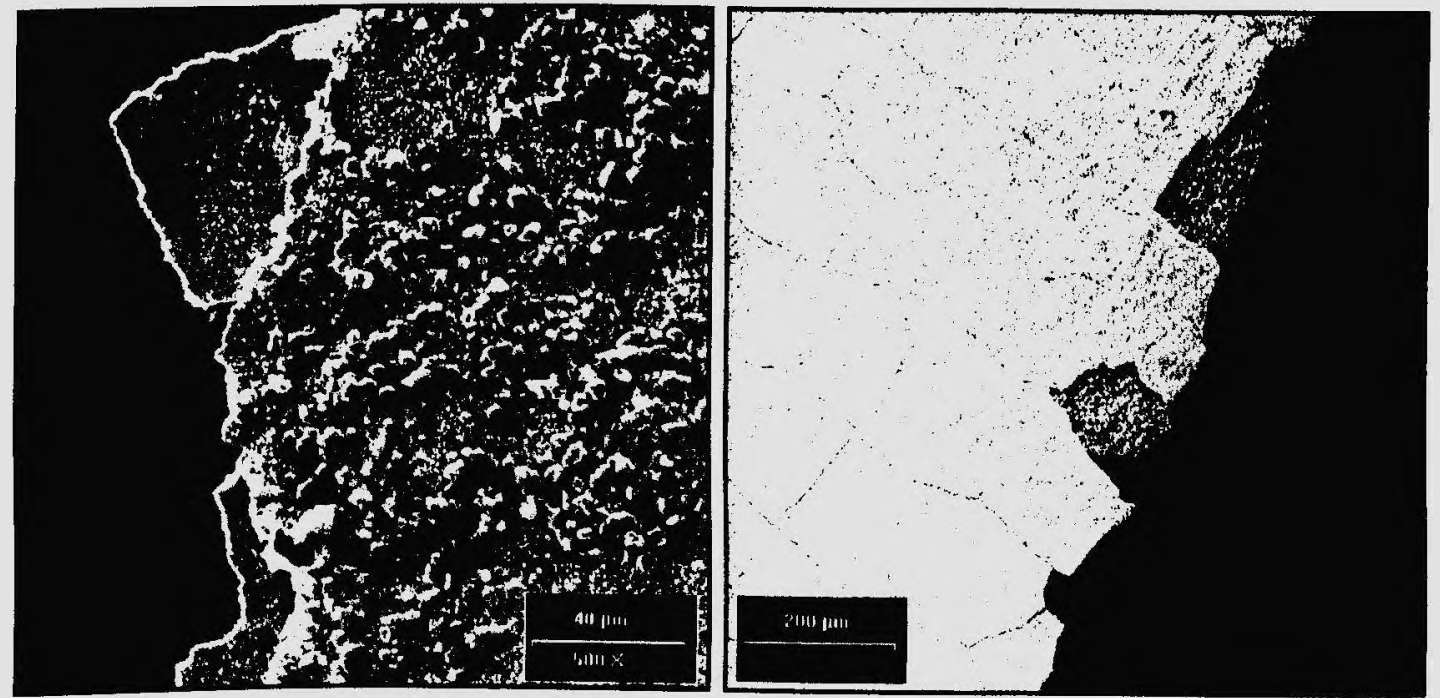

Figure A1: SEM micrographs of the spalled layer from the outer wall of the tube tested at 220 bar internal hydrogen pressure for 342 hours. Outermost layer on top (left), and innermost layer on top (right) 

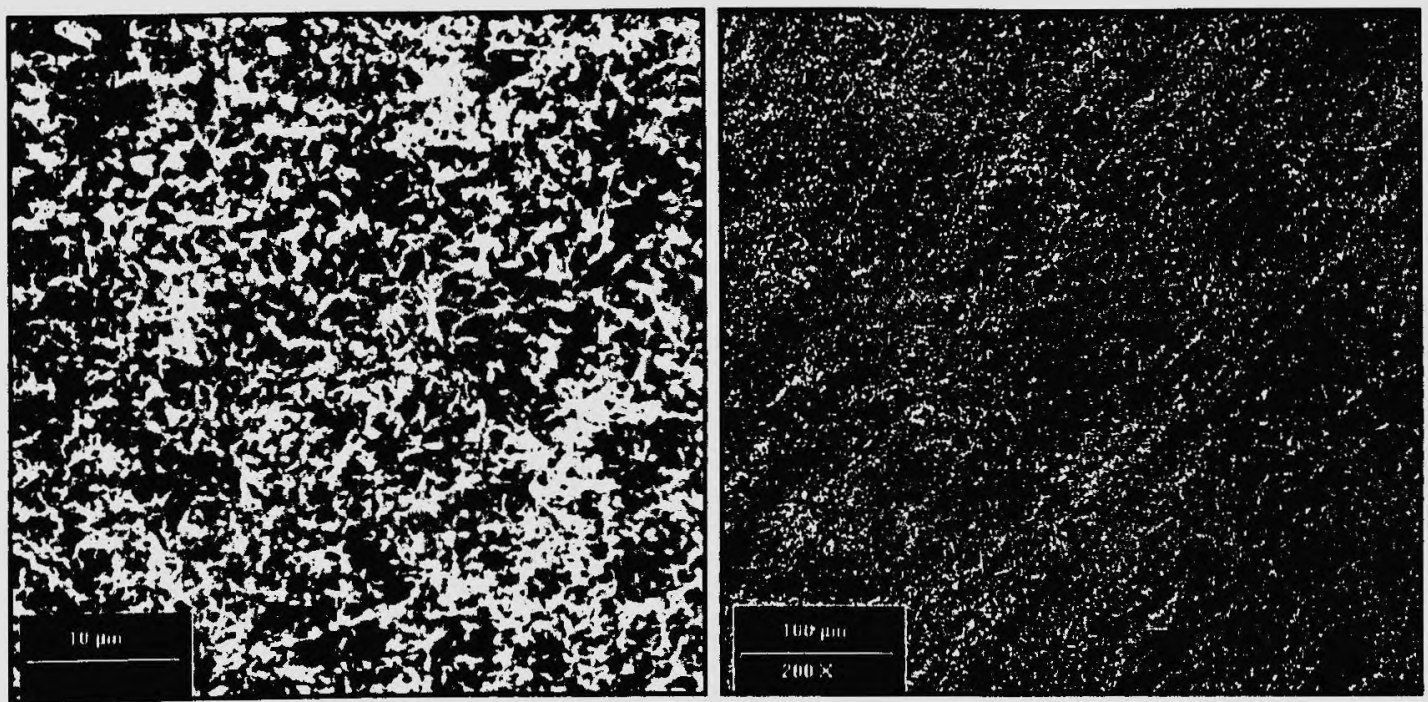

Figure A2: Close up SEM micrographs of the spalled layer from the outer wall of the tube tested at 220bar internal hydrogen pressure for 342hours. Porous outermost layer on top (left), and cracked innermost layer on top (right)

multilayer oxide consisting of magnetite $\left(\mathrm{Fe}_{3} \mathrm{O}_{4}\right)$ on the outside and spinel $(\mathrm{FeCr}$ Oxide) on the inside [Forres.' 80 ]. During tube testing, the tubes are surrounded by an argon blanket gas containing only a low oxygen partial pressure. Under these conditions, it seems reasonable that a thin double layer surface oxide can form, as described above. Magnetite has a black colour, which can explain the surface seen on argon pressurised tubes (fig. A3a).

However, under internal hydrogen pressure, it was observed that the outer layer had a lighter colour, with SEM analysis demonstrating that it consists of pure iron. It is suggested here that, during exposure, hydrogen can diffuse through the tube wall where it is able to reduce the outer layer of magnetite to pure iron, thereby creating the porous, lightly coloured outer surface. The spinel underside is not reduced, (at least not to the same degree) since the presence of high chromium and molybdenum contents help to stabilise the oxide. Finally, the reason why the surface tends to spall off may be because the diffusing hydrogen collects underneath the layer until the pressure causes it to fall away (fig. A3b).

The layer is important when using the capacitance gauges as the spallations can bridge the gap between the specimen and the gauges, thereby causing a short circuit and making the strain measurements meaningless. In addition, the layer may be important if it slows down the rate at which diffused hydrogen can leave the outer surface of the tube. In this case, the hydrogen concentration in the steel at the outer wall may be higher than otherwise expected. Normally, the hydrogen concentration is taken as zero at the outer wall. If this is false, then it may have consequences for hydrogen attack modelling and lifetime predictions. 


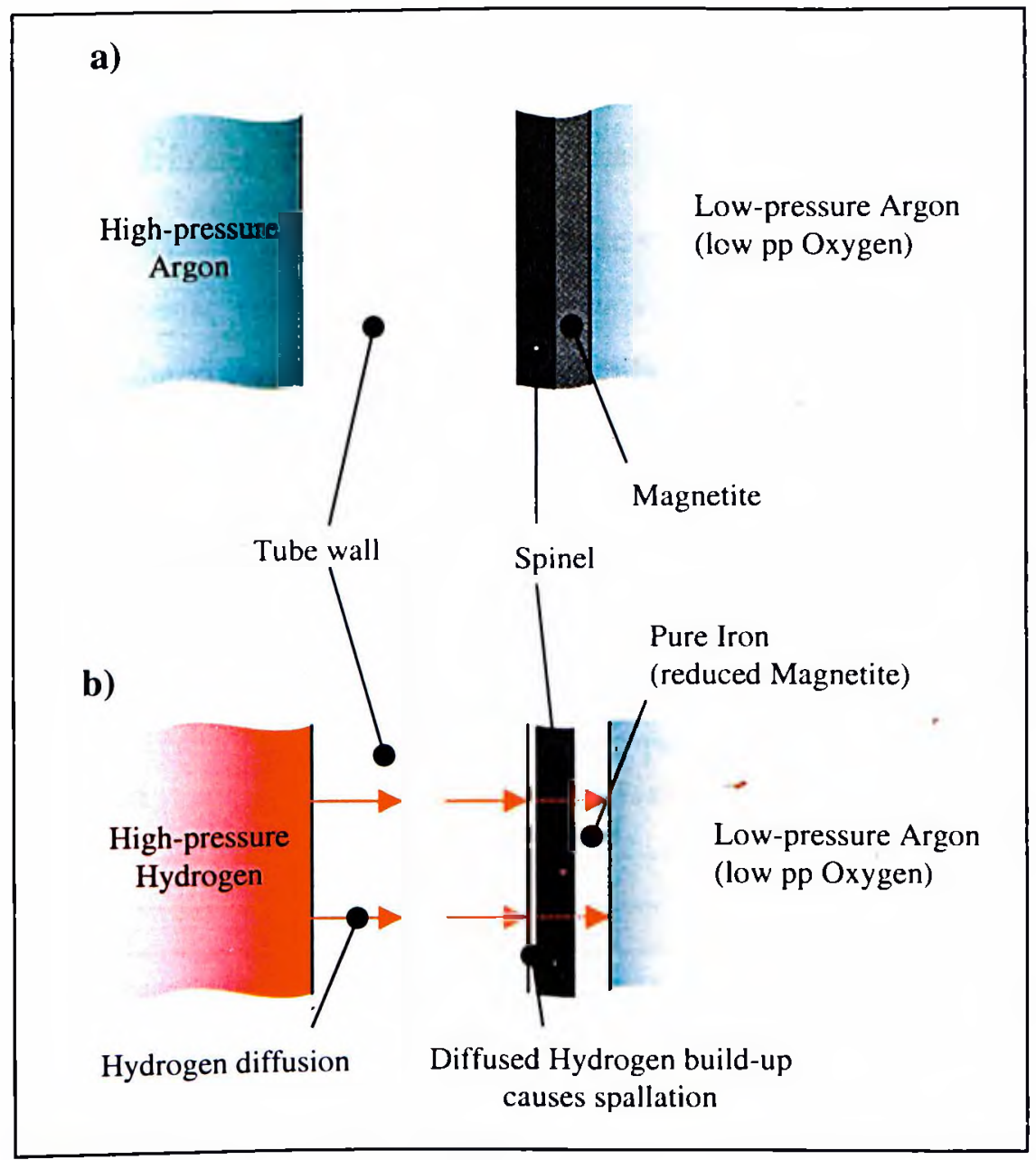

Figure A3: a) schematic illustration of the oxide layers forming during an internally pressurised tube test, with argon as the pressurising medium, and b) the reduction of these oxides and the spallation induced under internal hydrogen pressure 


\section{APPENDIX B}

\section{Capacitive Hoop Strain Measurement Validation}

The capacitive gauges used to measure the hoop strain of internally pressurised tubes had been carefully calibrated against known sized rods. However, calibration was not possible under conditions which simulated the surface changes described in Appendix A, nor was it practical to allow calibrations over long periods of time (several hundred hours) to test the stability of the device. To ensure that the strain measurements were accurate, tubes were measured manually with a micrometer after testing to compare against the final strain measured with the capacitive gauges. The comparison between the two sets of readings is given below in table BI.

\begin{tabular}{|c|c|c|}
\hline Tube Tested & $\begin{array}{c}\text { Maximum Average Hoop } \\
\text { Strain by Capacitance } \\
\text { Gauges (\%) }\end{array}$ & $\begin{array}{c}\text { Manual Micrometer Hoop } \\
\text { Strain Measurements (\%) }\end{array}$ \\
\hline $\mathrm{H}_{2} 260 \mathrm{bar}$ & 9.4 & 9.0 \\
\hline $\mathrm{H}_{2} 240 \mathrm{bar}$ & 25.4 & 26.3 \\
\hline $\mathrm{H}_{2} 220 \mathrm{bar}$ & 12.0 & 911 \\
\hline $\mathrm{H}_{2} 195 \mathrm{bar}$ & 9.5 & 9.3 \\
\hline $\mathrm{Ar} \mathrm{260} \mathrm{bar}$ & 8.0 & 9.0 \\
\hline $\mathrm{Ar} 240 \mathrm{bar}$ & 27.0 & 35.6 \\
\hline $\mathrm{Ar} 220 \mathrm{bar}$ & 10.5 & 10.7 \\
\hline
\end{tabular}

Table B1: Final hoop strains for internally pressurised tubes measured with the capacitance gauges, and with a micrometer after testing.

The table shows that in general the agreement between the capacitance gauges and the manually measured strains is good. The only significant difference occurs for the tube pressurised with 240 bar argon. This tube burst open at failure. At failure, the creep rate of this tube was very high, but data was only being logged by the extensometry every half hour. It is to be expected therefore that the final strain measurement is somewhat lower than the real strain. A similar problem was expected for the tube tested with 240 bar hydrogen but, as luck would have it, the tube burst shortly after a data point was logged so that little error is incurred. Normally, the logging interval is increased near to failure to prevent this problem from happening. However, when failures take place during the night or at the weekend, it is not possible to increase the logging rate. 


\section{APPENDIX C}

\section{Parameter Fitting of the CDM $\varepsilon^{-\mathrm{m}}$ Model}

$$
\frac{\mathrm{d} \varepsilon}{\mathrm{dt}}=\frac{\mathrm{K} \sigma^{\mathrm{n}}}{(1-\omega)^{\mathrm{n}}} \varepsilon^{-\mathrm{m}} \quad(\mathrm{Cl}) \quad \frac{\mathrm{d} \omega}{\mathrm{d} t}=\frac{A \sigma^{\nu}}{(1-\omega)^{\phi}} \varepsilon^{-\mathrm{m}}
$$

\section{Step 1}

Set $m=0$ and $\sigma=\sigma_{0}$, where $\sigma_{0}$ is the initial stress, and assume that failure occurs when $\omega=1$. This converts the model into a constant stress tertiary creep model, which can be easily solved to allow the parameters to be found analytically [Dunne'90]. Following this routine gives an initial starting set of parameters in a non subjective manner.

\section{Step 2}

Convert the model to a tertiary creep constant load model by keeping $\mathrm{m}=0$ and replacing $\sigma$ with $\sigma_{0}(1+\varepsilon)$. A strain time plot can then be found by splitting the creep curve up into lots of small increments of strain (or time) during which constant stress behaviour is assumed.

a) Start at $\mathrm{t}=0, \varepsilon=0.01 \%, \omega=0, \sigma=\sigma_{0}$ (N.B. strain cannot start at zero if $\mathrm{m}>0$, so a small initial strain is always assumed which could perhaps be considered as elastic strain)

b) Use equation $\mathrm{Cl}$ and the initial parameters $(\mathrm{K}, \mathrm{A}, \mathrm{n}, \mathrm{v}, \phi, \mathrm{m})$ to find the current strain rate $\mathrm{d} \varepsilon / \mathrm{dt}$.

c) A strain increment is chosen (often $0.025 \%$ ) which allows a time increment to be found: $\Delta \mathrm{t}=\frac{\Delta \varepsilon}{\mathrm{d} \varepsilon / \mathrm{dt}}$

d) The damage rate $\mathrm{d} \omega / \mathrm{dt}$ is found from equation $\mathrm{C} 2$ with the current paramters.

e) A damage increment is calculated for the current time increment by: $\Delta \omega=\frac{\Delta t}{d \omega / d t}$

f) Update the time, strain and damage with the increments $\Delta t, \Delta \varepsilon, \Delta \omega$. Calculate the new stress $\sigma=\sigma_{0}(1+\varepsilon)$.

g) Repeat the sequence from $b$ ) until the $\omega=\omega_{f}$, where $\omega_{f}$ is the damage at failure (normally considered as 1, but can be different: see Step 4)

The above sequence has been written into a MS Excel spreadsheet for ease of use, allowing the parameters to be changed quickly to see their effect on the curve shape. 


\section{Step 3}

The fitted curve will now be a conservative fit of the experimental data, having higher creep rates and lower times to failure. Adjust the parameters $K, A, n, v, \phi$ and re-run Step 2: a-g until the CDM and experimental curves fit better. In general $n$ should be $\geq v$ otherwise the strain to failure will increase with increasing test time. The greater the difference between $n$ and $v$, the greater the change in failure strain with test time. $v$ also has an effect on the slope of the rupture curve, whilst $A$ tends to effect the intercept of the rupture curve. $K$ and $n$ are used for fitting the creep rate in the early stages of the test. $\phi$ controls mainly the curvature of the creep curve. It is difficult to give each parameter a definite role because the constant load model is ratker nonlinear. However, the above guidelines are still helpful.

\section{Step 4}

Find the critical damage at failure $\omega_{\mathrm{f}}$, by fitting the curve shape better. When failure is assumed to occur at $\omega=1$ then the curve shape probably has too much curvature in the tertiary region, ie. the increase in creep rate is too sharp near to failure. By changing $\phi$ (perhaps the other parameters will need small adjustment after changing $\phi$ ), the curve shape can be better fitted. However, as the curve looses its sharpness, it will cause the failure strains to be too high, possibly several hundred percent. Choosing a suitable failure damage truncates the curves so that the strains to failure can be better fitted. This is a reasonable thing to do since omega is often described as being a pseudo loss of area, implying that $\omega=1$ represents necking to an infinitely long wire. Failure rarely occurs like this, rather damage progresses until some critical value when the material shears or tears in a sudden manner. Choosing a critical value of $\omega$ reflects this mechanism, as well as providing better curve fits.

\section{Step 5}

Fit primary creep. So far the curve fits have kept $m=0$, meaning no primary creep. Increasing $\mathrm{m}$ gives greater amounts of primary creep. By changing $\mathrm{m}$ from 0 , it will be necessary to change the other parameters again to maintain good curve fits. However, the other parameters should not have to change too much from their currently optimised values. This is one of the advantages of the $\varepsilon^{-\mathrm{m}}$ primary creep method over the $\mathrm{t}^{-\mathrm{m}}$ method. The $\varepsilon^{-\mathrm{m}}$ method requires less re-adjustment of the other parameters, probably because the strain range of each creep curve is similar, almost independent of test stress, whereas the time varies dramatically with stress. For 
the current work, primary creep is relatively small, so the fitting procedure works quite well and readjustment of the other parameters $K, A, n, v$, and $\phi$ is kept to a minimum. It may be necessary to repeat Step 4 to find a new critical damage. For creep curves with greater proportions of primary creep it may be better to start fitting $\mathrm{m}$ from the beginning since introduction of the parameter at this late stage could require much larger re-adjustments. 


\section{APPENDIX D}

\section{Calculation Procedure of The Thick Walled Concentric Cylinder Model for Predicting Hydrogen Pressurised Tube Creep Behaviour.}

\section{Concepts}

The model is based on the concentric tube model described for thin wall stress distributions in section 6.2. When using thick wall tubes, the stress distribution becomes more complex and must be calculated by assuming that the inner cylinder is pressurised from the inside $\left(\mathrm{P}_{\mathrm{i}}\right)$ and is constrained by an effective pressure on the outside $\left(\mathrm{P}_{\mathrm{o}}\right)$. The outer cylinder is considered to be under an internal pressure only, equal to $P_{0}$.

The stress distribution through the wall of the outer cylinder can be found from the Bailey equations for steady state creep [Bailey'51]:

$$
\sigma_{h}=P_{o} \frac{\frac{2-n}{n}\left(\frac{r_{o}}{r}\right)^{2 / n}+1}{\left(\frac{r_{o}}{r_{i}}\right)^{2 / n}-1} \quad \sigma_{r}=P_{o} \frac{1-\left(\frac{r_{o}}{r}\right)^{2 / n}}{\left(\frac{r_{o}}{r_{i}}\right)^{2 / n}-1} \quad \sigma_{a}=P_{o} \frac{\frac{1-n}{n}\left(\frac{r_{0}}{r}\right)^{2 / n}+1}{\left(\frac{r_{0}}{r_{i}}\right)^{2 / n}-1}
$$

Where $\sigma_{\mathrm{h}}, \sigma_{\mathrm{r}}$ and $\sigma_{\mathrm{a}}$ are the hoop, radial and axial stress; $r_{o}$ and $r_{i}$ are the outer and inner tube radii; $r$ is a radial position within the tube wall, $P_{o}$ is the pressure, and $n$ is the Norton law stress exponent of creep rate (not necessarily equal to $\mathrm{n}$ in the CDM equations). The stress distribution for the inner wall is calculated from equations for the steady state stress distribution derived from the above when there are both internal and externally applied pressures [Johnso.'61]:

$$
\sigma_{h}=\frac{P_{i}\left[\left(\frac{r_{i}}{r_{0}}\right)^{\frac{2}{n+1}}-\frac{(n-1)}{(n+1)}\left(\frac{r_{i}}{r}\right)^{\frac{2}{n+1}}\right]-P_{o}\left[1-\frac{(n-1)}{(n+1)}\left(\frac{r_{i}}{r}\right)^{\frac{2}{n+1}}\right]}{\left[1-\left(\frac{r_{i}}{r_{0}}\right)^{\frac{2}{n+1}}\right]}
$$




$$
\begin{aligned}
& \sigma_{n}=\frac{P_{i}\left[\left(\frac{r_{i}}{r_{0}}\right)^{\frac{2}{n+1}}-\frac{n}{(n+1)}\left(\frac{r_{i}}{r}\right)^{\frac{2}{n+1}}\right]-P_{s}\left[1-\frac{n}{(n+1)}\left(\frac{r_{i}}{r}\right)^{\frac{2}{n+1}}\right]}{\left[1-\left(\frac{r_{i}}{r_{0}}\right)^{\frac{2}{n+1}}\right]} \\
& \sigma_{r}=\frac{-P_{i}\left[\left(\frac{r_{i}}{r}\right)^{\frac{2}{n+1}}-\left(\frac{r_{i}}{r_{0}}\right)^{\frac{2}{n+1}}\right]-P_{0}\left[1-\left(\frac{r_{i}}{r}\right)^{\frac{2}{n+1}}\right]}{\left[1-\left(\frac{r_{i}}{r_{0}}\right)^{\frac{2}{n+1}}\right]}
\end{aligned}
$$

When using thick wall tubes the strain also varies across the wall, being higher at the tube bore than at the outer wall. The hoop strains at given radial positions can be calculated as shown in figure D1, where the dotted lines represent the tube after straining a given amount.

If $r_{i}$ and $r_{o}$ represent the radii before straining, and $R_{i}$ and $R_{0}$ represent the tube radii after a small amount of strain then, assuming no axial strain and no volume change, the cross sectional area of the tube remains the same, such that:

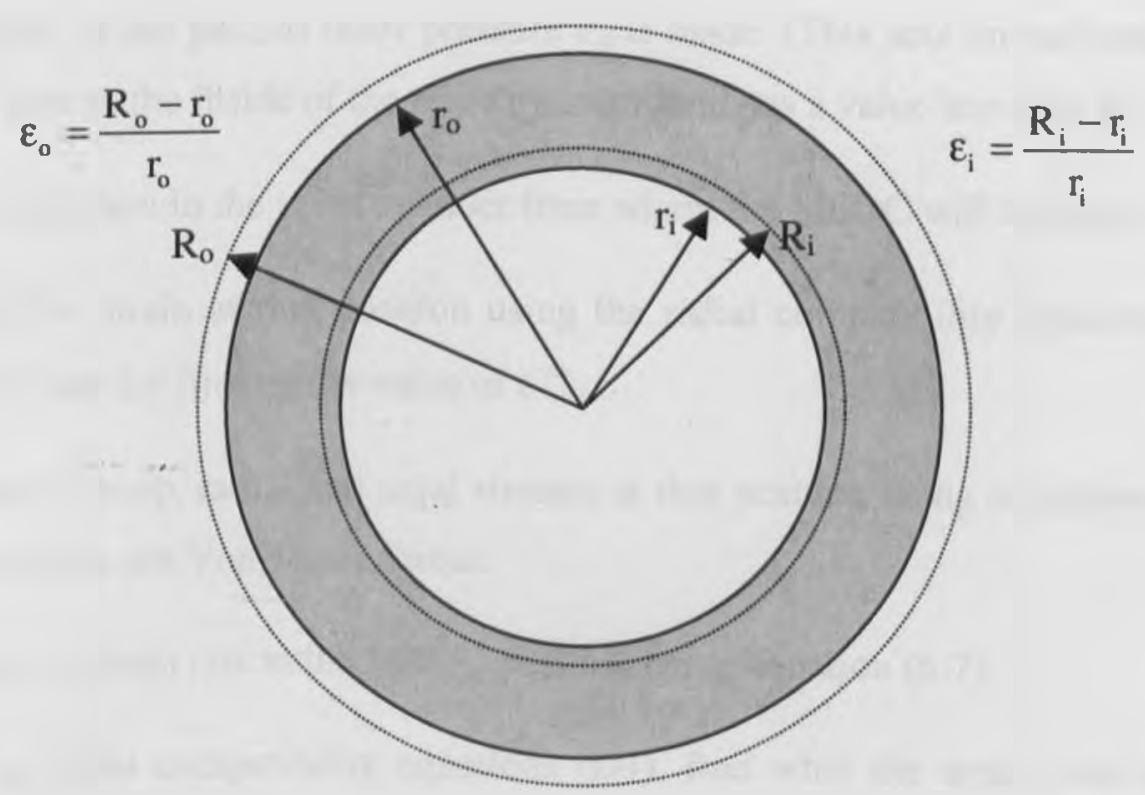

Figure D1: Strain calculations for given radial positions in a thick wall tube. 


$$
\pi r_{0}^{2}-\pi r_{i}^{2}=\pi R_{o}^{2}-\pi R_{i}^{2}
$$

i.e.

$$
r_{o}^{2}-r_{i}^{2}=R_{o}^{2}-R_{i}^{2}
$$

If the strain is known at a given radial position, then the new radius can be found out. By rearranging equation D6, the new radius (and hence strain) can be found at any other position. For example, when using the model, the strain on the outer tube surface is normally calculated, since this corresponds to the experimentally measured strain. This allows the new outer radius to be found $\left(R_{o}\right)$, from which the new tube inner radius $\left(R_{i}\right)$ can be derived as:

$$
R_{i}=\sqrt{R_{0}^{2}-r_{0}^{2}+r_{i}^{2}}
$$

N.B. the subscripts o and i do not necessarily have to refer to the outer and inner radii.

\section{Model Calculation Procedure}

1. Time, strain, inner and outer tube radii, interface position between the cylinders, damage parameters for both cylinders, and the internal pressure $\left(\mathrm{P}_{\mathrm{i}}\right)$ are known at the beginning of the calculation. (Strain on the outer wall is assumed to be $0.01 \%$ at the beginning of the test to avoid an error with the $\varepsilon^{-\mathrm{m}}$ function.)

2. An estimate of the pseudo outer pressure $P_{0}$ is made. (This acts on the outside of the inner cylinder and on the inside of the outer cylinder, and has a value less than $P_{i}$ )

3. Choose a position in the inner cylinder from where the MSDC will be taken.

4. Calculate the strain at that position using the radial compatibility equations (D7). This is necessary later for finding the value of $\varepsilon^{-m}$.

5. Calculate the hoop, radial and axial stresses at that position using equations (D2-D4). From these, calculate the Von Mises Stress.

6. Calculate the strain rate at the MSDC position using equation (6.7)

7. Using the radial compatibility equations (D7), find what the strain rate would be at the interface between the cylinders. (The strain rate is proportional to strain over small time increments).

8. Repeat steps 3-7 for the outer cylinder, using equations (D1) for the stresses. 
9. There should now be two strain rate predictions for the interface between the cylinders. Find the difference between them.

10. Adjust $P_{o}$ and repeat steps 3-9 until the difference between the strain rates is zero. (e.g. Use an error minimisation routine such as the MS EXCEL Goal Seek Function).

11. Using the radius compatibility equations (D7) calculate what the strain rate is at the outer tube wall.

12. Choose a strain increment (e.g. $\Delta \varepsilon=0.025 \%$ ), and from the strain rate at the outer tube wall, calculate the corresponding time increment: $\Delta t=\frac{\Delta \varepsilon}{\mathrm{d} \varepsilon / \mathrm{dt}}$

13. Choose a position and an $\alpha$ value for the MSRC in the inner cylinder. ( $\alpha$ controls the amount of MPS or Von Mises in the MSRC (equation(6.10)).

14. Calculate the current strain at this point (ignoring the most recently aquired strains above).

15. Calculate the hoop, axial, radial and corresponding Von Mises stresses at this postion.

16. Calculate the damage rate from equation (6.8).

17. The damage increment is found from $\Delta \omega=\frac{\Delta t}{\mathrm{~d} \omega / \mathrm{dt}}$, where $\Delta \mathrm{t}$ is determined in step 12 .

18. Repeat steps 13-17 for the outer wall.

19. Update the time, strain, inner and outer cylinder damage, inner tube radius, outer tube radius and radial position of the interface.

20. Repeat the next iteration from step 2 until the critical damage level is reached in either cylinder.

The above procedure has been computerised in a MS EXCEL spreadsheet, with a Visual Basic Macro used to trigger the Goal Seeking function for each iteration. 


\section{APPENDIX E}

\section{Determination of the Methane Pressure}

\section{Methane Pressure Calculation Method}

The following presents the method used for calculating the equilibrium methane pressure when the hydrogen pressure, temperature and carbon activity are known [Schlög.'99].

1. Convert the hydrogen pressure to units of atmospheres (e.g. multiply by 9.87 to convert MPa to Atmospheres).

2. Determine the carbon activity. For the current work, this was estimated by examining a chart of carbon activity vs tempering parameter for 2.25Cr-1 Mo steel [Partha.' 84 ].

3. The methane fugacity (in MPa) is then calculated from the following formula:

$$
f_{\mathrm{CH}_{4}}=0.101 \mathrm{a}_{\mathrm{c}} \mathrm{P}_{\mathrm{H}_{2}}^{2} \exp \left(\frac{-\Delta \mathrm{G}}{\mathrm{RT}}\right)
$$

where $a_{c}$ is the carbon activity, $P_{\mathrm{H}_{2}}$ is the hydrogen pressure, $\mathrm{R}$ is the universal gass constant, $\mathrm{T}$ is the temperature in kelvin and $\Delta \mathrm{G}$ is the Gibbs energy of formation for methane, defined as:

$$
\Delta \mathrm{G}=-69120-65 \cdot 35 \mathrm{~T}+51.25 \mathrm{~T} \cdot \log (\mathrm{T})
$$

4. The methane pressure is calculated using the methane fugacity-pressure relationship defined by Odette and Vagarali [Odette'82]:

$$
f=P \cdot \exp [C(T) \cdot P]
$$

where the value of $\mathrm{C}(\mathrm{T})$ depends on the fugactiy range being investigated:

$$
\begin{aligned}
& \text { for } \mathrm{f}<1000, \mathrm{C}(\mathrm{T})=0.005 \\
& \text { for } 1000<\mathrm{f}<10000, \mathrm{C}(\mathrm{T})=(1.1875 / \mathrm{T})+3.0888 \times 10^{-3} \\
& \text { for } 10000<\mathrm{f}, \mathrm{C}(\mathrm{T})=(2.2375 / \mathrm{T})+1.1776 \times 10^{-3}
\end{aligned}
$$

The fugacity-pressure relation can be solved by simple numerical techniques. 


\section{Methane Pressure Calculations for the Current Work}

\section{Methane Pressures for Given Applied Hydrogen Pressures}

To calculate the methane pressures for the current work, it was first necessary to find the carbon activity from the tempering parameter vs. activity chart in [Partha.'84]. The tempering parameter is defined as $T[20+\log (t)]$ where $T$ is the tempering temperature in Kelvin and $t$ is the tempering time in hours. The tempering treatment described in chapter 4 of 4 hours at $750^{\circ} \mathrm{C}$, gives a parameter of $21.08 \times 10^{3}$. Cross referencing this on the chart shown in gives a carbon activity of approximately 0.08 .

Using this carbon activity and a temperature of $600^{\circ} \mathrm{C}$, the following methane pressures were calculated for each of the hydrogen pressures used in the work of this thesis.

\begin{tabular}{|c|c|}
\hline Hydrogen Pressure [MPa] & Equilibrium Methane Pressure [MPa] \\
\hline 26 & 131.0 \\
\hline 24 & 118.7 \\
\hline 22 & 106.2 \\
\hline 19.5 & 90.4 \\
\hline
\end{tabular}

The internal methane pressures are of the order of the externally applied stresses. Due to the approximate method for determining the carbon activity, the methane pressure was recalculated for $22 \mathrm{MPa}$ hydrogen pressure using values of carbon activity of 0.06 and 0.1 to give an idea of the potential error in the pressure calculations. These gave methane pressures of 87.5 and 122.4 respectively.

\section{Calculating the Methane Pressure at a Given Point Within a Tube Wall}

To estimate the methane pressure at a given depth in the tube walls it was first necessary to calculate the effective hydrogen pressure at that position (given a gradient from the applied pressure at the inner, wall and zero at the outer wall). This was done by using the following equation [VdBurg'97]:

$$
\mathrm{P}_{\mathrm{H}_{2}}(\mathrm{r})=\mathrm{P}_{\mathrm{H}_{2}}(\mathrm{i})-\left\{\mathrm{P}_{\mathrm{H}_{2}}(\mathrm{o})-\mathrm{P}_{\mathrm{H}_{2}}(\mathrm{i})\right\} \frac{\ln \left[\mathrm{r} / \mathrm{r}_{\mathrm{o}}\right]}{\ln \left[\mathrm{r}_{\mathrm{i}} / \mathrm{r}_{\mathrm{o}}\right]}
$$

where $r(i)$ and $r(o)$ represent the inner and outer radii of the tube respectively, $P_{\mathrm{H}_{2}}(i)$ and $\mathrm{P}_{\mathrm{H}_{2}}$ (o) the hydrogen pressures at the inner and outer tube radii, and $\mathrm{P}_{\mathrm{H}_{2}}(r)$ represents the hydrogen pressure at a given radial position. 
In Chapter 7 there is interest in the methane pressure at a depth of $0.37 \mathrm{~mm}$. The hydrogen and methane pressures at such a position are shown in the table below.

\begin{tabular}{|c|c|c|}
\hline $\begin{array}{c}\text { Internal Hydrogen } \\
\text { Pressure [MPa] }\end{array}$ & $\begin{array}{c}\text { Hydrogen Pressure at } \\
\text { 0.37mm Depth [MPa] }\end{array}$ & $\begin{array}{c}\text { Methane Pressure at } \\
\text { 0.37mm Depth [MPa] }\end{array}$ \\
\hline 26 & 23.8 & 117.5 \\
\hline 24 & 22.0 & 106.2 \\
\hline 22 & 20.2 & 94.8 \\
\hline 19.5 & 17.9 & 80.1 \\
\hline
\end{tabular}




\section{REFERENCES}

[Allen'61] R.E. Allen, R.J. Jansen, P.C. Rosenthal, and F.H. Vitovec: "The Rate of Irreversible Hydrogen Attack Of Steel At Elevated Temperatures", Proc. API., vol. 41 [III], 1961, p74-85

[Allen'62] R.E. Allen, P.C. Rosenthal, F. H. Vitovec: "Creep Rupture Behaviour of Mild Steel Under Conditions of Hydrogen Attack", Proc. API., vol. 42 [III], 1962, p464-471

[Allen'62b] R.E. Allen, R.J. Jansen, P.C. Rosenthal, and F.H. Vitovec: "Analysis of Probable Mechanisms of High Temperature Hydrogen Attack of Steel", Proc. API., vol. 42 [III], 1962, p452-462

[Ansell'59] G.S. Ansell, J. Weertman: "Creep of a Dispersion-Hardened Aluminium Alloy", Trans. Of The Metallurgical Society of AIME, vol. 215, Oct. 1959, p838-843

[API'67] "Steel Deterioration in Hydrogen": API, div. of refining, 1967

[API'97]

API: "Steels for Hydrogen Service at Elevated Temperatures and Pressures in Petroleam Refineries and Petrochemical Plants", API recommended practice 941, Fith Edition, Jän 1997

[Argent'70] B.B. Argent, M.N. Niekerk, G.A. Redfern: "Creep of Ferritic Steels", J. Iron and Steel Inst., Sept. 1970, p830-843

[Argon'83] A.S. Argon: "Intergranular Cavitation in Creeping Alloys", Scipta Metall., vol. 17, 1983, p5-12

[Ashby'79] M.F. Ashby, C. Gandhi, D.M. Taplin: "Fracture Mechanism Maps and Their Construction for F.C.C. Metals and Alloys", Acta Metall., vol. 27, 1979, p699-729

[Ashby'84] M.F. Ashby, B.F. Dyson: "Creep Damage Mechanics and Micromechanisms", Proc. Int. Conf. Fracture ICF6, New Delhi, 1984, p3-30

[ASTM387] ASTM Standard A387: "Standard Specification for: Pressure Vessel Plates, Alloy Steel, Chromium-Molybdenum", Annual Book of ASTM Standards, Section 01.04, 1990

[Bailey'51] R.W. Bailey: "Steam Piping for High Pressures and High Temperatures", and "Creep Relationships and their Application to Pipes, Tubes and Cylindrical Parts under Internal Pressure", Proc. Instn mech. Engrs, London, vol. 164, 1951 ,p324, p425,

[Baker'59] R. Baker, J. Nutting: "The Tempering of 2.25Cr-IMo Steel after Quenching and Normalising", J. of the Iron and Steel Inst., vol. 192, July 1959, p257-268

[Beere'78] W. Beere, M.V. Speight: "Creep Cavitation by Vacancy Diffusion in Plastically Deforming Solid", Metal Science, April 1978, p172-176

[Beere'80] W. Beere: "Inhibition of Intergranular Cavity Growth in Precipitate-Hardened Materials", J. of Materials Science, vol. 15, 1980, p657-669

[Bhatta. ${ }^{25]}$ S. Bhattacharyya, R.H. Titran: "High Pressure Hydrogen Effect on Creep-Rupture Properties of Superalloys", J. Materials for Energy Systems, ASM, vol. 7, No. 2, Sept. 1985, p123-136

[Bina'96] V. Bina, O. Bielak, F. Hnilica: "Creep Behaviour and Structural Changes of Cr2.25Mol Steel After Long-Term Service in Hydrogen", eds. J. Petit, J. de Fouquet, G. Henaff, P. Villechaise, A. Dragon, Proc. $11^{\text {th }}$ Biennial European Conference on Fracture - ECF 11, Poitiers, France, 3-6 Sept., 1996, p873-878

[Braam'94] H. Braam, B.R.W. Haverkate: "Creep Lifetime Prediction of Pressurised Tubes with Continuum Damage Mechanics", Conference proceedings from: "ECF 10: Structural Integrity: Experiments-Models-Applications", Berlin, EMAS, 20-23 Sept 1994, p279-284

[Bramle.'32] A. Bramley, K.F. Allen: "The Loss of Carbon From Iron and Steel When Heated in Decarburising Gases", Engineering, vol. 133, Jan. 22, 1932, p92-94

[Britis.'86] ASTM Standard A335: "Standard Specification for: Seamless Ferritic Alloy-Steel Pipe For High-Temperature Service". (from) Iron and Steel Specifications, $6^{\text {th }}$ edition, British Steel Corp., 1986, p216

[Brouwe.'92] R.C. Brouwer: "Hydrogen Diffusion and Solubility in Vanadium Modified Pressure Vessel Steels", Scripta Metall., vol. 27, 1992, p353-358

[BS3500] British Standard BS 3500: British Standard Methods For: "Creep and Rupture Testing of Metals", Part 3. Tensile Creep Testing, 1969

[BS3604] British Standard BS 3604: Steel Pipes and Tubes for Pressure Purposes: Ferritic Alloy Steel with Specific Elevated Temperature Properties, (1978) 
[Burke'75] M.A. Burke, W.D. Nix: "Plastic Instabilities in Tension Creep", Acta Metall., vol. 23, July 1975, p793-798

[Campbe.'23] E.D. Campbell, J.F. Ross, W.L. Fink: "The Relative Efficiency of Dry and of Moist Hydrogen on the Decarburisation of Steel at $950^{\circ} \mathrm{C}$, and the Effect of Hydrogen on the Phosphorous Content.", J. of the Iron and Steel Inst., No. II, vol. CVIII, 1923, p179-188

[Cane'79] B.J. Cane: "Interrelationship Between Creep Deformation and Creep Rupture in 2.25Cr-IMo Steel", Metal Science, May 1979, p287-294

[Cane'81] B.J. Cane: "Mechanistic control regimes for intergranular cavity growth in 2.25Cr-1Mo steel under various stresses and stress states", Metal Science, vol. 15, July 1981, p302-310

[Cane'8lb] B.J. Cane: “Creep Damage Accumulation And Fracture Under Multiaxial Stresses", Proc. $5^{\text {th }}$ Int. Congr. On Fracture, Cannes, France, 1981, p1285-1293

[Cane'82] B.J. Cane: "Remaining Creep Life Estimation by Strain Assessment on Plant", Int. J. Press. Ves. \& Piping, vol. 10, 1982, p 11-30

[Cane'82b] B.J. Cane, R.J. Browne: "Representative Stresses for Creep Deformation and Failure of Pressurised Tubes and Pipes", Int. J. Pres. Ves. \& Piping, vol. 10, 1982, p1 19-128

[Cane'85] B.J. Cane, R.D. Townsend: "Flow and Fracture at Elevated Temperatures", Ed. Raj, ASME, Metals Park, Ohio, 1985, p147-177

[Canjar'67] L.N. Canjar and F.S. Maning: "Thermodynamic Properties and Reduced Correlations for Gases", Gulf publishing Co., Houston, TX, 1967

[Chaboc. '74] J.-L. Chaboche: "Une Loi Différentielle D'Endommagement de Fatigue Avec Cumulation Non Linéaire", Revue Française de Méchanique, No. 50-51, 1974, p71-82

[Chaboc.'81] J.-L. Chaboche: "Continous Damage Mechanics - A Tool to Describe Phenomena Before Crack Initiation", Nuclear Eng. And Design, 64, North Holland Publ. Co., 1981, p233-247

[Chen'95] L-C. Chen, P. Shewmon: "Stress-Assisted Hydrogen Attack Cracking in 2.25Cr-IMo Steels at Elevated Temperatures", Metall. And Materials Trans., vol.26A, Sept. 1995, p2317-2327

[Chuang'73] T.J. Chuang, J.R. Rice: "The Shape of Intergranular Creep Cracks Growing by Surface Diffusion", Acta. Metall., vol. 21, Dec. 1973, p1625-1628

[Chuang'79] T.J. Chuang, K.I. Kagawa, J.R. Rice and L.B. Sills: "Non-Equilibrium Models for Diffusive Cavitation of Grain Interfaces", Acta Metall., vol. 27, 1979, p265

[Chung'82] D.W. Chung, J.A. Todd, J.K. Youngs, E.R. Parker: "Hydrogen Attack of Thick Section Pressure Vessel Steels", Proc. Conf. Pressure Vessels \& Piping, Orlando, ASME MPG-18, 1982, p25-52

[Church'92] J.M. Church: "Creep and Creep Crack Growth Behaviour of Ferritic Tubular Components Under Multiaxial Stress States", PhD. Thesis, Dept. of Mech. And Proc. Eng., University of Sheffield, Nov. 1992

[Ciuffr.82] A.R. Cuiffreda, N.B. Heckler, E.B. Norris: "Stress-Rupture Behaviour of Cr-Mo Steels in a High Pressure Hydrogen Environment", Proc. Conf. Pressure Vessels and Piping, Orlando, ASME MPG-18, 1982, p53-68

[Clugst.'83] S.F. Clugston, J. R. Weertman, P. Shewmon: "The Enhancement of Hydrogen Attack in Steel by Prior Deformation", Metall. Trans. A, vol. 14A, 1983, p695-699

[Coble'63] R.L. Coble: "A Model for Boundary Diffusion Controlled Creep in Polycrystalline Materials", Journal of Applied Physics, vol. 34, no. 6, June 1963, p1679-1682

[Cocks'82] A.C.F. Cocks, M.F. Ashby: "On Creep Fracture by Void Growth", Progress in Materials Science, vol. 27, 1982, p189-244

[Cocks'82b] A.C.F. Cocks, M.F. Ashby: "Creep Fracture by Coupled Power-Law Creep and Diffusion Under Multiaxial Stress", Metal Science, vol. 16, Oct. 1982, p465-474

[COST'94] COST 501-11 Work Package 5C: "Prediction of Lifetime Under Service Loads", Final Report, Ed. P.J.L. Meriguet, March 1994

[Cuiffr.'82] A.R. Cuiffreda, N.B. Heckler, E.B. Norris: "Stress Rupture Behaviour of Cr-Mo Steels in a High-Pressure Hydrogen Environment", Proc. Conf. On Press. Vess. And Piping, Orlando, ASME MPG-18, 1982, p53-68 [Davies'73] P.W. Davies, C. Nelmes, K.R. Williams, B. Wilshire: "Stress-Change Experiments during High-Temperature Creep of Copper, Iron and Zinc", Metal Sci. J., vol. 7, 1973, p87-92 
[Davis'59] E.A. Davis, F.M. Connelly: "Stress Distribution and Plastic Deformation in Rotating Cylinders of Strain Hardening Material", J. Applied Mechanics, Trans. ASME, March, 1959, p25-30

[DMIC'64] Defense Metals Information Center: "The Effects of High-Pressure, H-Temperature Hydrogen on Steel", DMIC report 202, March 26, 1964

[Dorn'54] J.E. Dorn: "Some Fundamental Experiments on High Temperature Creep", J. of the Mechanics and Physics of Solids, vol. 3, 1954, p85-116

[Dunne'90] F.P.E. Dunne, A.M. Othman, F.R. Hall, D.R. Hayhurst: "Representation of Uniaxial Creep Curves Using Continuum Damage Mechanics”, Int. J. Mech. Sci., vol. 32, No. 11, 1990, p945. 957

[Dyson'73] B.F. Dyson, D.E. Henn: "The Effect of Room Temperature Pre-Strain on Grain Boundary Cavitation in Nimonic 80A", Journal of Microscopy, vol. 97, Pts 1/2, Jan./March 1973, pl65-170

[Dyson'76] B.F. Dyson: "Constraints on Diffusional Cavity Growth Rates", Metal Science, October 1976 , p349-352

[Dyson'76b] B.F. Dyson: "Gıain Boundary Cavitation Under Various States of Applied Stress", Proc. R. Soc. Lond., A, vol. 349, 1976, p245-259

[Dyson'83] B.F. Dyson: "Continuous Cavity Nucleation and Creep Fracture”, Scripta Metall., vol. 17, 1983, p31-37

[Edward'79] G.H. Edward, M.F. Ashby: "Intergranular Fracture During Power-Law Creep", Acta Metall., vol. 27, p $1505-1518$

[ENEL'97] ENEL-DSR/EC-DG-JRC-IAM Research Contract: "Material Requirements for Improved Design and Operation in High Temperature Components for Power Plants", Final Report, European Commission JRC., SCI unit, JRC Contract No. 40.0016, Nov. 1997

[Erwin'82] W.E. Erwin, J.G. Kerr: "The Use of Quenched and Tempered 2.25Cr-IMo Steel For Thick Wall Reactor Vessels in Petroleum Refinery Processes: An Interpretive Review of 25 Years of Research and Application", Welding Research Council Bulletin 275, 1982

[Evans'82] R.W. Evans, J.D. Parker, B. Wilshire: "Recent Advances in Creep and Fracture of Engineering Materials and Structures", eds. Wilshire and Owen, Pineridge Press, 1982, pl35184

[Evans'85] R.W. Evans, B. Wilshire: "Creep of Metals and Alloys", The Institute of Metals, London, 1985, p202

[Fields'80] R.J. Fields, T. Weerasooriya, M.F. Ashby: "Fracture Mechanisms in Pure Iron, Two Austenitic Steels, and one Ferritic Steel”, Metall. Trans., vol. I1 A, Feb. 1980, p333-347

[Finnie'59] I. Finnie, W. Heller: “Creep of Engineering Materials”, McGraw Hill, New York, 1959

[Forres. '80] J.E. Forrest, P.S. Bell: "The Relationship Between the Deformation of Substrate and Scale in theMechanism of Multilayer Oxide Formation on Low Alloy Ferritic Steels", Proc. Of the European Symp. On "Corrosion and Mechanical Stress at High Temperatures", Eds. V. Guttmann, M. Merz, Petten, NL, May 1980

[Furtad.'97] H.C. Furtado, I. Le May: "Modelling of Creep Damage to Estimate Remaining Life", Mat. Sci. and Eng., A234-236, 1997, p87-90

[Garofa.'65] F. Garofalo: "Fundamentals of Creep and Creep-Rupture in Metals", The Macmillan Co. New York, Editors: M.E. Fine, J. Weertman, J.R. Weertman, 1965

[Geiger'75] F. H. Geiger, O. F. Angelis: API pub. No. 945, 1975

[Goods'83] S.H. Goods, T.G. Nieh: "Mechanisms of Intergranular Cavity Nucleation and Growth During Creep", Scripta Metall., vol. 17, 1983, p23-30

[Gope'92] N. Gope, T. Mukherjee, D.S. Sarma: "Influence of Long-Term Aging at $520^{\circ} \mathrm{C}$ and $560^{\circ} \mathrm{C}$ and Superimposed Creep Stress on the Microstructure of 1.25Cr-0.5Mo Steel", Metall. Trans., vol. 23A, January 1992, p221-235

[Grabke'72] H.J. Grabke, E. Martin: "Kinetik und Thermodynamik der Auf-und Entkohlung von $\alpha$-Eisen in $\mathrm{CH}_{4}$ - $\mathrm{H}_{2}$-Gemischen", Arch. Eisenhüttenwes., 44, nr. 11, 1972, p837-842

[Harris’73] J.E. Harris: “The Inhibition of Diffusion Creep by Precipitates", Metal Sci. J., vol. 7, 1973

[Hayhur.'78] D.R. Hayhurst, F.A. Leckie, C.J. Morrison: Proc. R. Soc., London, A360, 1978, p243-264 
[Hayhur.'84] D.R. Hayhurst, P.R. Dimmer, C.J. Morrson: "Development of Contimuum Damage in The Creep Rupture of Notched Bars", Phil. Trans. R. Soc. Lond., A 311, 1984, p103-129

[Hayhur.'85] D.R. Hayhurst, D.A. Lavender: "An Assessment of the A-Projection Method for the Representation and Extrapolation of Creep Data for a $1 \% \mathrm{Cr}, 1 / 2 \% M o, 1 / 4 \% \mathrm{~V}$ Steel Tested at $565^{\circ} C^{\prime \prime}$, Int. J. Pres. Ves. \& Piping, vol. 20, 1985, p289-317

[Hender. '82] J. Henderson, B.F. Dyson: "Measurement of High Temperature Mechanical Properties", eds. Loveday, Day and Dyson, HMSO, 1982, p171-184

[Herrin.'50] C. Herring: "Diffusional Viscosity of a Polycrystalline Solid", J. Applied Physics, vol. 21, May 1950, p437-445

[Hopkin'62] L.M.T. Hopkin, E.A. Jenkinson: "Creep Resistance and Metallographic Structure of 2.25CrIMo Steel With and Without a Tin Addition”, J. of the Iron and Steel Inst., May 1962, p356-359

[HTMTC'89] High Temperature Mechanical Testing Committee: "A Code of Practice for Internal Pressure Testing of Tubular Components at Elevated Temperatures", Prepared by the Internal Pressure Testing Working Party, HTMTC/IP/6/89, April, 1989

[Hull'59] D. Hull, D.E. Rimmer: "The Growth of Grain Boundary Voids Under Stress", Phil. Mag., vol. 4, 1959, p673

[Hurst'98] R.C. Hurst, J.H. Rantala, F. Bregani: "Application of Creep Models for Lifetime Prediction of Ferritic Power Plant Steels", PVP 1988, ASME/JSME Joint Pressure Vessel and Piping Conf., Severe Accidents, San Diego, California, July 26-30, 1988

[Hutchi.'83] J.W. Hutchinson: "Constitutive Behaviour and Crack Tip Fields For Materials Undergoing Creep-Constrained Grain Boundary Cavitation", Acta Metall., vol. 31, No. 7, 1983, p1079-1088

[Ishigu.'94] T. Ishiguro, K. Kimura, T. Hatakeyama, T. Tahara: "Effect of Metallurgical Factors on Hydrogen Attack Resistance in C-0.5Mo Steel". $2^{\text {nd }}$ Int. Conf. On Interaction of Steels With Hydrogen in Petroleum Industry Pressure Vessel and Pipeline Service, Vienna, Austria, Oct. 1994, p367-377

[ISO'81] ISO: "Summary of Average Stress Rupture Properties of Wrought Steels for Boilers and Pressure Vessels", Technical Report 7468, Ref. No. ISO/TR 7468-1981 (E)

[Johnso.'51] A.E. Johnson: :"Creep Under Complex Stress Systems at Elevated Temperature", Proc. Instn. Mech. Engrs., Lond., vol. 164, 1951, p432-447

[Johnso.'60] A.E. Johnson, J. Henderson, B. Khan: “Complex Stress Creep, Relaxation and Fracture of Metallic Alloys", HMSO, Edinburgh, 1960

[Johnso.'61] A.E. Johnson, J. Henderson, B. Khan: "Behaviour of Metallic Thick Walled Cylindrical Vessels or Tubes Subject to High Internal or External Pressures at Elevated Temperatures", Proc. Inst. Mech. Engrs., vol. 175, No. 25, 1961, p1043-1072

[Kachan.'58] L.M. Kachanov: IZV. AKAD. NAVK. SSSR. O. T. N. Techkh. NAVK, 8, 1958, p20

[Kanaza.'77] S. Kanazawa, Y. Otoguro, T. Horiya, H. Nakoa, N. Tanaka, R. Yamaba: "2.25Cr-1Mo Steel Plate with Improved Temper Embrittlement Characteristics", $3^{\text {rd }}$ Int. Conf. On Pressure Vessel Technol., ASME, Tokyo, Part 2, 1977, p1003-1010

[Karzov'94] G.P. Karzov, G.N. Filimonov, JU.I. Archakov, A.V. Gribanov, JU.S. Medvedev: "High Parameter Hydrogen Effects on Creep Rupture Strength of the Advanced 3Cr-Mo-V Steel Used for Pressure Vessels of Cracking Reactors", Second Int. Conf. on Interaction of Steels with Hydrogen in Petroleum Industry Pressure Vessel and Pipeline Service, Vienna, Austria, Oct. 1994, p389-394

[Kleuh'74] R.L. Kleuh: "The Effect of Carbon on 2.25Cr-IMo Steel (I). Microstructure and Tensile Properties", J. of Nuclear Materials, 54, 1974, p41-54

[Kolgat.'59] N.N. Kolgatin, L.A. Glikman, V.P. Teodorovich, V.I. Deryabina: “Rupture Strength of Tubular Steel Specimens under Internal Hydrogen Pressure at Elevated Temperatures", Metallovedenie ie i Termich. Obrabokta Metallov, March 1959, no. 3, p19-24

[Lame $\left.{ }^{18} 52\right] \quad$ G. Lame: "Lecons sur la Theorie de l'Elasticitie", Paris, 1852

[Larson'52] F.R. Larson, J. Miller: Trans. ASME, vol. 74, 1952, p765-775

[Leckie'74] F.A. Leckie, D.R. Hayhurst: "Creep Rupture of Structures", Proc. R. Soc. Lond., A340, 1974, p323-347 
[Leckie'74b] F.A. Leckie, A.R.S. Ponter: "On the State Variable Description of Creeping Materials", Ingenieur-Archiv 43, Springer Verlag, 1974, p158-167

[Leckie'75] F.A. Leckie, D.R. Hayhurst: "The Damage Concept in Creep Mechanics", Mech. Res. Comm., vol. 2, 1975, p23-26

[Loghma.'96] A. Loghman \& M.A. Wahab: "Creep Damage Simulation of Thick-Walled Tubes Using the Theta Projection Concept", Int. J. Press. Ves. \& Piping, vol. 67, 1996, p105-111

[Looney'94] L. Looney: "The Effect of High Pressure Hydrogen on the Creep Fracture of Ferritic Steel Components", PhD. Thesis, University of Dublin, Trinity College, May 1994

[Lopez'83] H. Lopez, P. Shewmon: "Precipitation Induced Voids in Quenched and Tempered Steel", Acta Metall., vol. 31, 1983, 1945-1950

[Lundbe.'77] R. Lundberg, M. Waldenstrom, B. Uhrenius, CALPHAD 1, 159, 1977

[Lundin'94] C. Lundin, K. Khan, P. Liu: "Study of Hydrogen Attack Susceptibility of 2.25Cr-IMo Steel", $2^{\text {nd }}$ Int. Conf. On Interaction of Steels With Hydrogen in Petroleum Industry Pressure Vessel and Pipeline Service, vol. 1, Vienna, Austria, 1994, p331

[Manjoi.'75] M.J. Manjoine: "Ductility Indices at Elevated Temperature", Trans. Of the ASME, J. of Eng. Materials and Tech., April 1975, p156-161

[Manola.'98] P. Manolatos: (Private Communication), SIC Unit, JRC of the E.C., Institute for Advanced Materials, Petten, The Netherlands.

[Masaok. '77] I. Masaoka, I. Takase, S. Ikeda, R. Sasaki: J. Jap. Wëld. Soc., 1977, vol. 46, p818-824

[Masaok.'82] I. Masaoka, K. Kinoshita, R. Chiba, T. Fujii, T. Imanaka, T. Mitsuo, Y. Otoguro, S. Kihara, H. Miyaji, T. Sakai: "Hydrogen Attack Limit of 2.25Cr-IMo Steel", from "Current Solutions to Hydrogen Problems in Steel", ASM, Metals Park, OH, 1982, p242-248

[Masuya. '90] F. Masuyama, N. Nishimura, Y. Takeda: "Carbide Changes and Sulphur Segregation in CrMo Steel During Creep", High Temp. Tech., vol. 8, No. 4, Nov. 1990, p257-260

[McKimp.'81] M. McKimpson and P. Shewmon: "Initial Hydrogen Attack Kinetics in a Carbon Steel", Metall. Trans. A, vol 12A, 1981, p825-834

[Metals'48] Metals Handbook, 1948 Edition, Am. Soc. Metals, Cleveland, Ohio, p 1208

[Mitsuo'82] T. Mitsuo, K. Yamato, T. Saitoh: "Effects of a Simulated Weld Thermal Cycle and Post Weld Heat Treatment on the Hydrogen Attack of 2.25Cr-1Mo Steel", Proc. Conf. Pressure Vessels \& Piping, Orlando, ASME, MPG-18, 1982, p69-83

[Monkma.'56] F.C.Monkman, N.J. Grant: “An Empirical Relationship Between Rupture Life and Minimum Creep Rate in Creep-Rupture Tests", Proc. ASTM., vol. 56, 1956, p593-620

[Mullan.'67] J.A. Mullandore, J.P. Tralmer: "Hydrogen Attack in Cr-Mo Steels", 32 ${ }^{\text {nd }}$ Mid Year Meeting, API Division of Refinery, 1967, p429-446

[Murphy'71] M.C. Murphy, G.D. Branch: "Metallurgical Changes in 2.25CrMo steels during Creep Rupture Test", J. of the Iron and Steel Inst., vol. 209, July 1971, p546-561

[Nabarr.'48] F.R.N. Nabarro: "Deformation of Crystals by the Motion of Single lons", Report on a conference on the strength of solids, The Physical Society, London, 1948, p75-90

[Needha.'80] N.G. Needham, T. Gladman: "The Effect of Stress State on the Processes Controlling Creep Fracture in a 2.25Cr-1Mo Steel", Int. Conf. On Eng. Aspects of Creep, I. Mech. E., Sheffield, vol. $1,1980, \mathrm{p} 49-54$

[Nix'89] W.D. Nix, J.C. Earthman, G. Eggeler, B. Ilschner: "The Principal Facet Stress as a Parameter for Predicting Creep Rupture Under Multiaxial Stresses", Acta Metall., vol. 37, No. 4, 1989, p1067-1077

[Norris'76] E.B. Norris, E.A. Sticha: "Effect of Hydrogen on the Stress-Rupture Strength of 2.25Cr-1Mo Steel", International Joint Petroleum Mechanical Engineering and Pressure Vessel and Piping Conference, Mexico City, Sept. 1976, ed. A. Schaefer

[Norton'39] F.H. Norton: Trans. ASME., vol. 61, 1939, p239

[Nuovo]

Nuovo Pignone, Manufacturer of heavy reactors for hydrocracking, hydrotreating and similar refinery applications, Commercial documentation, Florence, Italy.

[Odette'82] G.R. Odette and S.S. Vagarali: "An Equation of State for Methane for Modelling Hydrogen Attack in Ferritic Steels", Metall. Trans. A, vol 13A, 1982, p299-303 
[Odette'82b] G. Odette: "Analysis of Hydrogen Attack on Pressure Vessel Steels", Final Rept., California Univ., Santa Barbara, Dept. of Chem. And Nuclear Eng., 1982

[Okumur.'76] M. Okumura, M. Kumagai, H. Nakamura, K. Kohira: "Electroslag Welding of Heavy Section 2.25Cr-IMo Steel", Welding Research Supplement, Dec. 1976, p389-399s

[Orr'54] R.L. Orr, O.D. Sherby, J.E. Dorn: “Correlations of Rupture Data For Metals At Elevated Temperatures", Trans. Of the ASM, vol. 46, 1954, pl13-128

[Panda'84] B. Panda and P.G. Shewmon: "Kinetics of Bubble Growth in a l020 Steel", Metall. Trans. A, vol. 15A, 1984, p487-494

[Partha.'84] T.A. Parthasarathy and P.G. Shewmon: "Effects of Tempering on the Carbon Activity and Hydrogen Attack Kinetics of 2.25Cr-1Mo Steel", Metall. Trans. A, vol.15A, 1984, p2021-2027

[Partha.'85] T.A. Parthasarathy and P.G. Shewmon: "Hydrogen Attack Kinetics of 2.25Cr-1Mo Steel Metal Welds", Metall. Trans., vol. 16A, 1985, p1143-1149

[Partha.'85b] T.A. Parthasarathy: "Mechanisms of Hydrogen Attack of Carbon and 2.25Cr-IMo Steels", Acta Metall., vol. 33, 1985, p/673-168I

[Pendse'85] R.D. Pendse, R.O. Ritchie: "A Study of Fatigue Crack Propagation in Prior Hydrogen Attack Pressure Vessel Steels", Metall. Trans., vol. 16A, Aug. 1985, p1491-1501

[Pishko'79] R. Pishko, M. McKimpson, P. Shewmon: "The Effect of Steelmaking on the Hydrogen Attack of Carbon Steel", Metall. Trans. A, vol. 10A, 1979, p887-894

[Rabotn. '69] Y.N. Rabotnov: "Creep Problems in Structural Members", North Holland, Amsterdam, 1969

[Raj'75] R. Raj, M. Ashby: “Intergranular Fracture at Elevated Temperature”. Acta Metall., vol. 23, 1975, p653-666

[Raj"77] R. Raj, H. Shih, H. Johnson: "Correction to: Intergranular Fracture at Elevated Temperature", Scripta Metall., vol. 11, 1977, p839-842

[Ransic.'81] M. Ransick, P. Shewmon: "Effect of Cold Work on Hydrogen Attack", Metall. Trans. A, vol. 12A, 1981, p17-22

[Riedel'89] H. Riedel: "Life Prediction Methods for Constrained Grain Boundary Cavitation", Int. J. Pres. Ves. \& Piping, vol. 39, 1989, pl19-134

[Robins.'52] E.L. Robinson: "Effect of Temperature Variation on the Long-Time Rupture Strength of Steels", Trans. ASME, vol. 74, 1952, p777

[Rosent.'63] P.C. Rosenthal, W.L. Schroeder, F.H. Vitovec: "Effect of Low-Alloy Additions on the Resistance of Carbon Steels to Hydrogen Attack”, Proc. API., vol. 43 [III], 1963, p98-105

[Ruoff'87] S. Ruoff, D. Stone, C.-Y. Li: "Hydrogen Attack in a 3Cr-1.5Mo Steel at Elevated Temperatures", Mat. Sci. and Eng., vol. 93, 1987, p217-225

[Sachs'70] K. Sachs, A.J. Baldock, D.G. Evans: "The Creep Strength of Welds in Thick Steampipes", Proc. Of the Conf. On Welding of Creep Resistant Steels, The Welding Institute, Cambridge, 1970, p105-114

[Sagues'78] A.A. Sagues, B. Hall, H. Wiedersich: "On the Mechanisms of Hydrogen Attack", Scripta Metall, vol. 12, 1978, p319-326

[Sakai'80] T. Sakai, H. Kaji: "Nucleation and Growth of Bubbles Formed by Hydrogen Attack in Low Alloy Steels", Proc. Conf. On Hydrogen in Metals, Minikami, (1979), Jap. Inst. Metals, 1980. p489-492

[Sakai"81] T. Sakai, H. Kaji: "Effect of Impurity Elements on the Formation of Bubbles by Hydrogen Attack in 2.25Cr-IMo Steel", Proc. Conf. On Hydrogen Effects in Metals, Moran, Wyo., USA.,(1980),TMS/AIME 1981, p903-911

[Schlög.'99] S. Schlögl: (Private Communication), Dept. Microcmechanics of Materials, Technical University of Delft, Delft, The Netherlands

[Sdobyr.'58] C.R. Sdobyrev: IZK. AKAD. NAVK. SSSR. Otd. Tekh. Nauk., 4, 1958

[Shamma.'87] M.S. Shammas: Conf. Proc. "Life Extension and Refurbishment of Steam Plant", I. Mech. E., London, 1987, p289-299

[Shamma.'87b] M.S. Shammas: "Remanent Life Assessmeni of Ferritic Weld Heat Affected Zones by a Metallographic Measurement of Cavitation Damage - the 'A' Parameter", Conf. "Refurbishment and Life Extension of Steam Plant", 14-15 Oct, IMechE, 1987, p289-299 
[Sherby'67] O.D. Sherby, P.M. Burke: "Mechanical Behaviour of Crystalline Solids at Elevated Temperature", Progress in Materials Science, vol. 13, No. 7, Pergammon Press, 1967

[Shewmo.'76] P.G. Shewmon: "Hydrogen Attack of Carbon Steel”, Metall. Trans,, vol. 7A, Feb. 1976, p279. 286

[Shewmo.'80] P.G. Shewmon, C. Richied, M. McKimpson, M. Ransick: "Effect of Hydrogen Pressure on Morphology of Hydrogen Attack", Scripta Metall., vol. 14, 1980, p545-548

[Shewmo.'82] P.G. Shewmon, Z-S. Yu: "Hydrogen Attack Kinetics in 2.25Cr-IMo Pressure Vessel Steel", Proc. Conf. Pressure Vessels \& Piping, Orlando, ASME, MPG-18, 1982, p85-92

[Shewmo.'83] P.G. Shewmon, H. Lopez, T.A. Parthasarathy: "Chemically Driven Cavity Growth", Scripta Metall., vol. 17, 1983, p39-43 [Shewmo.'85]P.G. Shewmon: "Critical Assessment: Hydrogen attack of pressure vessel steels", Mater. Sci. Tech., vol. 1, 1985, p2-11

[Shewmo.'87] P.G. Shewmon: "Synergism Between Creep Ductility and Grain Boundary Bubbles", Acta Metall., vol. 35, No. 6, 1987, p1317-1324

[Shewmo.'91] P.G. Shewmon: "Effect of High-Pressure Hydrogen on Crack Growth in Carbon Steel", Metall. Trans., vol. 22A, Nov. 1991, p2703-2707

[Shih'82] H.M. Shih, H.H. Johnson: "A Model Calculation of the Nelson Curves For Hydrogen Attack", Acta metall., vol. 30, 1982, p537-545

[Shimom.'85] j. Shimomura, T. Imanaka: "Decomposition of Carbides in 2.25Cr-1Mo Steels During Hydrogen Attack", Scripta Metall., vol. 19, 1985, p1507-1511

[Soderb.'36] C.R. Soderberg: "The Interpretation of Creep Tests for Machine Design", Trans. ASME., vol.58, 1936, p733-743

[Soderb. '41] C.R. Soderberg: "The Interpretation of Creep Tests on Tubes", Trans. ASME, vol. 63, 1941

[Sponse.'66] D.L. Sponseller, F.H. Vitovec: "Resistance to Hydrogen Attack at looOF of 2.25\%Cr-1\%Mo and $0.5 \% \mathrm{Mo}-0.006 \% B$ Steels", Symp. Heat Treated Steels for Elevated Temperature Service, ASME, 1966, p74-85

[Stamm'93] H. Stamm, U. von Estorff: “Determination of Damage Parameters for Microcrack Formation Under Creep Conditions", from "Behaviour of Defects at High Ternperatures", ESIS 15, Eds. R.A. Ainsworth and R.P. Skelton, Mechanical Engineering Publications, London, 1993, p123151

[Sticha'69] E.A. Sticha: "Tubular Stress-Rupture Testing of Chromium Molybdenum Steels With High Pressure Hydrogen", J. of Basic Engineering, vol. 91, Trans. Of The ASME, New York, Dec. 1969, p590-592

[Stone'65] P.G. Stone, J.D. Murray: "Creep Ductility of Cr-Mo-V Steels", J. Iron and Steel Inst., Nov. 1985, p 1094-1107

[Su'84] Z. Su, D. Stone, J. Wanagel, C-Y. Li: "Hydrogen Attack in a low Si-0./C-2.25Cr-IMo-.25V-TiB Steel", Mat. Sci. Eng., vol. 62, 1984, p216-264

[Sundar.'80] G. Sundararajan, P. Shewmon: "The Hydrogen Attack of HSLA Steels", Metall. Trans., vol. $11 \mathrm{~A}, 1980, \mathrm{p} 509-516$

[Sundar.'81] G. Sundararajan, P. Shewmon: "The Kinetics of Hydrogen Attack of Steels", Metall. Trans., vol. 12A, 1981, p1761-1775

[Suther.'65] C.W. Sutherland, P.J. Vacca, P.C. Rosenthal, F.H. Vitovec: "Effect of Stress Concentration and Gas Pressure on the Creep Strength of Steel in Hydrogen Environment", Proc. API., vol. 45, [III], 1965, p181-189

[Tagawa'84] H. Tagawa, S. Tsuyama, H. Suzuki, N. Abe, M. Yamada, M. Hayashida: "Modified 3Cr-IMo Steel with Improved Toughness and Elevated Temperature Strength", Nippon Kokan Technical Report, Overseas No. 41, 1984, p31-40

[Taira'68] S. Taira, R. Ohtani: "Creep Rupture of Internally Pressurised Cylinders at Elevated Temperatures", Bulletin of JSME, vol. 11, No. 46, 1968, p593-601

[Taylor'87] N.G. Taylor: "The Multiaxial Creep Behaviour of Alloy $800 \mathrm{H}$ at $800^{\circ} \mathrm{C}$ in Carburising Environments", PhD. Thesis, University of Dublin, Trinity College, 1987

[Taylor'90] N.G. Taylor, B.R. Twaddle, R.C. Hurst: "Prediction of Alloy $800 \mathrm{H}$ and $2.25 \mathrm{Cr}-1 \mathrm{Mo}$ Component Behaviour From Uniaxial Specimen Data", Proc. $4^{\text {th }}$ Int. Conf. On Creep and Fracture of Engineering Materials and Structures, eds. B. Wilshire, R. Evans, 1990, p985-998 
[Thoma'66] P.E. Thoma, F.H. Vitovec, J.A. Mullandore: "Effect of Hydrogen on the Creep Rupture Properties of Iron", Proc. API, vol. 46 [III], 1966, p345-35]

[Thyseg. '64] J.R. Thygeson, M.C. Molstad: "High Pressure Hydrogen Attack of Steel", J. of Chem. And Eng. Data, vol. 9, No. 2, April 1964, p309-315

[Tresca $\left.{ }^{18} 64\right] \quad$ M. Tresca: C.R. ACAD. Sci., Paris, 59, 1864, p2 754

[VdBurg'94] M.W.D. Van Der Burg, E. Van Der Giessen: "Hydrogen Attack in Creeping Polycrystals due to Cavitation on Grain Boundaries", Proc. $5^{\text {th }}$ Int. Conf. On the Effects of Hydrogen on Material Behaviour, Eds. A.W. Thompson, N.R. Moody, TMS, Warrendale, PA, 1994, p313

[VdBurg'96] M.W.D. Van Der Burg, E. Van Der Giessen, R.C. Brouwer: "Investigation of Hydrogen Attack in 2.25Cr-IMo Steels with a High Triaxiality Void Growth Model", Acta mater, vol 44, No. 2, 1996, p505-518

[VdBurg'96b] M.W.D. Van Der Burg, E. Van Der Giessen: "Non Uniform Hydrogen Attack and the Role of Interaction with Creep", Mat. Sci. and Eng., A 220, 1996, p200

[VdBurg'97] M.W.D. Van Der Burg: "Hydrogen Attack: Micromechanical Modelling on Three Length Scales", Thesis, Delft University of Technology, 1997

[VdGies.'95] E. Van Der Giessen, M.W.D. Van Der Burg, A. Needleman, V. Tvergaard: "Void Growth due to Creep and Grain Boundary Diffusion at High Triaxialities", .J. of the Mechanics and Physics of Solids 43, 1995, p123

[Vitove.'64] F.H. Vitovec: "The Growth rate of Fissures During Hyärogen Attack of Steels", Proc. API., vol. 44 [III], 1964, p179-188

[VonMis.'13] R. Von Mises: "Mechanik der Festen und Flussigen Korper in Plastisch Deformablen Zustland", Kungl. Ges. Der Wiss., Gottingen, 1913

[Wada'82] T. Wada, T.B. Cox: "3Cr-1.5Mo Steel for Pressure Vessels in Hydrogen Service", Proc. Conf. Pressure Vessels \& Piping, Orlando, ASME MPG-18, 1982, p111-121

[Wada'83] T.Wada, V.A. Biss: "Restoration of Elevated Temperature Tensile Strength in 2.25Cr-IMo Steel", Metall. Trans., vol. 14A, May 1983, p845-855

[Wanage.'82] J. Wanagel, T. Hakkarainen, C.-Y. Li: “Hydrogen Attack in Thick Section 2.25Cr-IMo Steels at Elevated Temperatures", Application of $2.25 \mathrm{Cr}-1 \mathrm{Mo}$ Steel for Thick Wall Pressure Vessels., ASTM STP 755, Eds. G. Sangdahl, M. Semchysen, 1982, p93-108

[Weertm.'55] J. Weertman: "Theory of Steady-State Creep Based on Dislocation Climb", Journal of Applied Physics, vol. 26, no. 10, Oct. 1955, p1213-1217

[Weertm.'57] J. Weertman: "Steady State Creep through Dislocation Climb", Journal of Applied Physics, vol. 28, no. 3, Mar. 1957, p362-364

[Weertm.'60] J. Weertman: Trans. AIME, (218), 1960, p207

[Weiner'61] L.C. Weiner: "Kinetics and Mechanisms of Hydrogen Attack of Steel", Corrosion, vol. 17, 1961, p137t-143t

[Westph.'74] D.A. Westphal, F.J. Worzala: "Hydrogen Attack of Steel", Proc. Conf. "Effects of Hydrogen on Materials Properties and Selection”, eds. I.M. Bernstein \& A.W. Thompson, ASM, 1974, p79-89

[Woodhe.'65] J.H. Woodhead, A.G. Quarrell: "Role of Carbides in Low Alloy Creep Resisting Steels", J. Iron and Steel Inst., June 1965, p605-620

[Woods'83] C.M. Woods, T.E. Scott: "Hydrogen Attack of Bainitic 2.25Cr-IMo Steel", eds. DeHoff, Braun, McCall, Microstructural Science, Vol. 11, 1983, p465-479

[Yokoga.'78] K. Yokogawa, S. Fukuyama, M. Mitsui, K. Kudo: "Pressure Vessel for Tensile Testing in High-Pressure gases at Elevated Temperatures", Rev. Sci. Instrum., vol. 49(1), Jan. 1978, p5051

[Yokoga.'84] K. Yokogawa, S. Fukuyama, K. Kudo, M. Araki: "Hydrogen Damage of 2.25Cr-lMo Reactor Steel Under Constant Loading in High Pressure Hydrogen At Elevated Temperatures", $5^{\text {th }}$ Int. Conf. On Pressure Vessel Technology, vol. II, San Francisco, California, Sept. 1984

[Yokoga.'89] K. Yokogawa, S. Fukuyama, K. Kudo, P. Shewmon: "Effect of Hydrogen Attack on Tensile and Creep Properties of Low Carbon Steel", Int. J. Pres. Ves. \& Piping, vol. 37, 1989, p365-385

[Yu'89] J. Yu: "Carbide Stability Diagrams in 2.25Cr-IMo Steels", Communications, Metall. Trans. A, vol. 20A, August 1989, p1561-1563 